

\section{Algebra \& Number Theory}

www.jant.org

\section{EDITORS}

\author{
MANAGING EDITOR \\ Bjorn Poonen \\ University of California \\ Berkeley, USA
}

\author{
EDITORIAL BOARD CHAIR \\ David Eisenbud \\ University of California \\ Berkeley, USA
}

\section{BOARD OF EDITORS}

$\begin{aligned} \text { Georgia Benkart } & \text { University of Wisconsin, Madison, USA } \\ \text { Dave Benson } & \text { University of Aberdeen, Scotland } \\ \text { Richard E. Borcherds } & \text { University of California, Berkeley, USA } \\ \text { John H. Coates } & \text { University of Cambridge, UK } \\ \text { J-L. Colliot-Thélène } & \text { CNRS, Université Paris-Sud, France } \\ \text { Brian D. Conrad } & \text { University of Michigan, USA } \\ \text { Hélène Esnault } & \text { Universität Duisburg-Essen, Germany } \\ \text { Hubert Flenner } & \text { Ruhr-Universität, Germany } \\ \text { Edward Frenkel } & \text { University of California, Berkeley, USA } \\ \text { Andrew Granville } & \text { Université de Montréal, Canada } \\ \text { Joseph Gubeladze } & \text { San Francisco State University, USA } \\ \text { Ehud Hrushovski } & \text { Hebrew University, Israel } \\ \text { Craig Huneke } & \text { University of Kansas, USA } \\ \text { Mikhail Kapranov } & \text { Yale University, USA } \\ \text { Yujiro Kawamata } & \text { University of Tokyo, Japan } \\ \text { János Kollár } & \text { Princeton University, USA } \\ \text { Hendrik W. Lenstra } & \text { Universiteit Leiden, The Netherlands } \\ \text { Yuri Manin } & \text { Northwestern University, USA } \\ \text { Barry Mazur } & \text { Harvard University, USA }\end{aligned}$

Susan Montgomery

Shigefumi Mori

Andrei Okounkov

Raman Parimala

Victor Reiner

Karl Rubin

Peter Sarnak

Michael Singer

Ronald Solomon

Vasudevan Srinivas

J. Toby Stafford

Bernd Sturmfels

Richard Taylor

Ravi Vakil

Michel van den Bergh

Marie-France Vignéras

Kei-Ichi Watanabe

Andrei Zelevinsky

Efim Zelmanov
University of Southern California, USA

RIMS, Kyoto University, Japan

Princeton University, USA

Emory University, USA

University of Minnesota, USA

University of California, Irvine, USA

Princeton University, USA

North Carolina State University, USA

Ohio State University, USA

Tata Inst. of Fund. Research, India

University of Michigan, USA

University of California, Berkeley, USA

Harvard University, USA

Stanford University, USA

Hasselt University, Belgium

Université Paris VII, France

Nihon University, Japan

Northeastern University, USA

University of California, San Diego, USA

\section{PRODUCTION}

ant@mathscipub.org

Paulo Ney de Souza, Production Manager

Silvio Levy, Senior Production Editor

See inside back cover or www.jant.org for submission instructions.

Regular subscription rate for 2008: $\$ 180.00$ a year (\$120.00 electronic only).

Subscriptions, requests for back issues from the last three years and changes of subscribers address should be sent to Mathematical Sciences Publishers, Department of Mathematics, University of California, Berkeley, CA 94720-3840, USA.

Algebra \& Number Theory, ISSN 1937-0652, at Mathematical Sciences Publishers, Department of Mathematics, University of California, Berkeley, CA 94720-3840 is published continuously online. Periodical rate postage paid at Berkeley, CA 94704, and additional mailing offices.

\section{PUBLISHED BY \\ 5 mathematical sciences publishers \\ http://www.mathscipub.org \\ A NON-PROFIT CORPORATION \\ Typeset in LATEX}

Copyright (C) 2010 by Mathematical Sciences Publishers 


\title{
Homology and cohomology of quantum complete intersections
}

\author{
Petter Andreas Bergh and Karin Erdmann \\ Dedicated to Luchezar Avramov on the occasion of his sixtieth birthday
}

\begin{abstract}
We construct a minimal projective bimodule resolution for every finite-dimensional quantum complete intersection of codimension two. Then we use this resolution to compute both the Hochschild cohomology and homology for such an algebra. In particular, we show that the cohomology vanishes in high degrees, while the homology is always nonzero.
\end{abstract}

\section{Introduction}

The notion of quantum complete intersections originates from the work by Manin [1987], who introduced the concept of quantum symmetric algebras. These algebras were used by Avramov, Gasharov and Peeva [1997] to study modules behaving homologically as modules over commutative complete intersections. In particular, they introduced quantum regular sequences of endomorphisms of modules, thus generalizing the classical notion of regular sequences.

Benson, Erdmann and Holloway [2007] defined and studied a new rank variety theory for modules over finite-dimensional quantum complete intersections. For this theory to work, it is essential that the commutators defining the quantum complete intersection be roots of unity, so that a linear combination of the generators behave itself as a generator. In this setting, at least for quantum complete intersections of codimension two, the Hochschild cohomology ring is infinite-dimensional, and a priori there might be connections between rank varieties and the support varieties defined by Snashall and Solberg [2004] (see also [Erdmann et al. 2004]).

Whether or not the higher Hochschild cohomology groups of a finite-dimensional algebra of infinite global dimension can vanish, known as "Happel's question", was unknown until the appearance of [Buchweitz et al. 2005]. In that paper, the authors constructed a four-dimensional selfinjective algebra whose total

MSC2000: primary 16E40; secondary 81R50, 16U80, 16S80.

Keywords: quantum complete intersection, Hochschild cohomology, Hochschild homology. The authors are grateful for support through the EC contract MRTN-CT-2003-505078 (LieGrits). This work was carried out while Bergh was working at the University of Oxford. 
Hochschild cohomology is five-dimensional, thus giving a negative answer to Happel's question. The algebra they constructed is the smallest possible noncommutative quantum complete intersection.

In this paper we study finite-dimensional quantum complete intersections of codimension two. For such an algebra, we construct a minimal projective bimodule resolution, and use this to compute the Hochschild homology and cohomology. In particular, we show that the higher Hochschild cohomology groups vanish if and only if the commutator element is not a root of unity, whereas the Hochschild homology groups never vanish. Thus we obtain a large class of algebras having the same homological properties as the algebra used in [Buchweitz et al. 2005].

\section{The minimal projective resolution}

Throughout this paper, let $k$ be a field and $q \in k$ a nonzero element. In the main results, this element is assumed not to be a root of unity, implying indirectly that $k$ is an infinite field. We fix two integers $a, b \geq 2$, and denote by $A$ the $k$-algebra

$$
A=k\langle X, Y\rangle /\left(X^{a}, X Y-q Y X, Y^{b}\right) .
$$

This is a finite-dimensional algebra of dimension $a b$, and it is justifiably a quantum complete intersection of codimension 2 ; it is the quotient of the quantum symmetric algebra

$$
k\langle X, Y\rangle /(X Y-q Y X)
$$

by the quantum regular sequence $X^{a}$ and $Y^{b}$ (as defined in [Avramov et al. 1997, Section 2]). We denote the generators of $A$ by $x$ and $y$, and use the set

$$
\left\{y^{i} x^{j}\right\}_{0 \leq i<b, 0 \leq j<a}
$$

as a $k$-basis. The opposite algebra of $A$ is denoted by $A^{\text {op }}$, and the enveloping algebra $A \otimes_{k} A^{\text {op }}$ by $A^{\mathrm{e}}$.

We now construct explicitly a minimal projective bimodule resolution

$$
\mathbb{P}: \cdots \rightarrow P_{2} \stackrel{d_{2}}{\rightarrow} P_{1} \stackrel{d_{1}}{\rightarrow} P_{0} \stackrel{\mu}{\rightarrow} A \rightarrow 0
$$

in which $P_{n}$ is free and of rank $n+1$, viewing the bimodules as left $A^{\mathrm{e}}$-modules. The generators $1 \otimes 1$ of $P_{n}$ are labeled $\epsilon(i, j)$ for $i, j \geq 0$, such that

$$
P_{n}=\bigoplus_{i+j=n} A^{\mathrm{e}} \epsilon(i, j) .
$$


For each $s \geq 0$, define the four elements of $A^{\mathrm{e}}$ :

$$
\begin{aligned}
& \tau_{1}(s)=q^{s}(1 \otimes x)-(x \otimes 1) \\
& \tau_{2}(s)=(1 \otimes y)-q^{s}(y \otimes 1) \\
& \gamma_{1}(s)=\sum_{j=0}^{a-1} q^{j s}\left(x^{a-1-j} \otimes x^{j}\right) \\
& \gamma_{2}(s)=\sum_{j=0}^{b-1} q^{j s}\left(y^{j} \otimes y^{b-1-j}\right) .
\end{aligned}
$$

Let $P_{0} \stackrel{\mu}{\rightarrow} A$ be the multiplication map $w \otimes z \mapsto w z$. The kernel of this map is generated by $\tau_{1}(0)$ and $\tau_{2}(0)$. Now let $R_{1}$ and $R_{2}$ be the commutative subalgebras of $A$ generated by $x$ and $y$, respectively. The annihilator of $\tau_{i}(0)$, viewed as an element of $R_{i}^{\mathrm{e}}$, is $\gamma_{i}(0)$, and the complex

$$
\cdots \rightarrow R_{i}^{\mathrm{e}} \stackrel{\tau_{i}(0)}{\longrightarrow} R_{i}^{\mathrm{e}} \stackrel{\gamma_{i}(0)}{\longrightarrow} R_{i}^{\mathrm{e}} \stackrel{\tau_{i}(0)}{\longrightarrow} R_{i}^{\mathrm{e}} \stackrel{\mu}{\rightarrow} R_{i} \rightarrow 0
$$

is a minimal projective bimodule resolution of $R_{i}$ [Holm 2000].

In general, given any algebra $\Gamma$ and an automorphism $\Gamma \stackrel{\psi}{\rightarrow} \Gamma$, we may endow every $\Gamma$-module $X$ with a new module structure by restricting scalars via $\psi$. In this way, we obtain a new module ${ }_{\psi} X$, whose underlying set is the same as that of $X$, but where scalar multiplication is given by

$$
\gamma \cdot x=\psi(\gamma) x
$$

for $\gamma \in \Gamma$ and $x \in X$. This new module is the twist of $X$ with respect to $\psi$. A homomorphism $X \rightarrow Y$ of $\Gamma$-modules induces a homomorphism

$$
{ }_{\psi} X \rightarrow{ }_{\psi} Y
$$

of twisted modules.

Now for $i=1,2$, define an algebra automorphism $R_{i}^{\mathrm{e}} \stackrel{\sigma_{i}}{\rightarrow} R_{i}^{\mathrm{e}}$ by

$$
\begin{aligned}
& \sigma_{1}: x \otimes 1 \mapsto x \otimes 1,1 \otimes x \mapsto q(1 \otimes x), \\
& \sigma_{2}: y \otimes 1 \mapsto q(y \otimes 1), 1 \otimes y \mapsto 1 \otimes y .
\end{aligned}
$$

When we twist the above resolution of $R_{i}$ by the automorphism $\sigma_{i}^{s}$ for some $s \geq 0$, then multiplication by $\tau_{i}(0)$ and $\gamma_{i}(0)$ become multiplication by $\tau_{i}(s)$ and $\gamma_{i}(s)$, respectively. We denote this twisted resolution by $\mathbf{R}_{i}(s)$. 
We now define a double complex

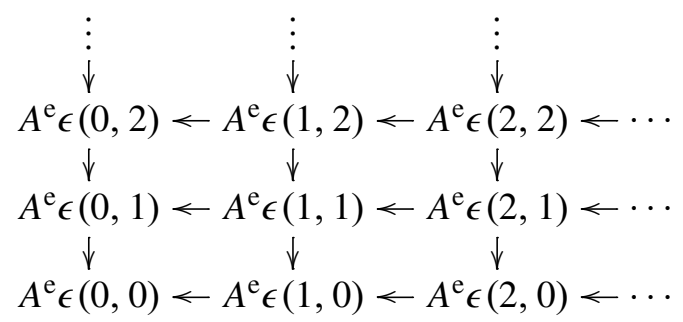

whose total complex $\mathbb{P}$ turns out to be the projective bimodule resolution we are seeking. Along row $2 s$ we use the resolution $\mathbf{R}_{1}(b s)$, and along row $2 s+1$ we use the resolution $\mathbf{R}_{1}(b s+1)$. Explicitly, the row maps are given by

$$
\begin{aligned}
\epsilon(2 r, 2 s) & \mapsto \gamma_{1}(b s) \epsilon(2 r-1,2 s), \\
\epsilon(2 r+1,2 s) & \mapsto \tau_{1}(b s) \epsilon(2 r, 2 s), \\
\epsilon(2 r, 2 s+1) & \mapsto \gamma_{1}(b s+1) \epsilon(2 r-1,2 s+1), \\
\epsilon(2 r+1,2 s+1) & \mapsto \tau_{1}(b s+1) \epsilon(2 r, 2 s+1) .
\end{aligned}
$$

Similarly, along column $2 r$ we use the resolution $\mathbf{R}_{2}(a r)$, and along column $2 r+1$ we use the resolution $\mathbf{R}_{2}(a r+1)$, introducing a sign in the odd columns. The column maps are therefore given by

$$
\begin{aligned}
\epsilon(2 r, 2 s) & \mapsto \gamma_{2}(a r) \epsilon(2 r, 2 s-1), \\
\epsilon(2 r, 2 s+1) & \mapsto \tau_{2}(a r) \epsilon(2 r, 2 s), \\
\epsilon(2 r+1,2 s) & \mapsto-\gamma_{2}(a r+1) \epsilon(2 r+1,2 s-1), \\
\epsilon(2 r+1,2 s+1) & \mapsto-\tau_{2}(a r+1) \epsilon(2 r+1,2 s) .
\end{aligned}
$$

It is straightforward to verify that these maps indeed define a double complex; all the four different types of squares commute. The transpose of the matrices defining the maps in the resulting double complex are given by

$$
\left(\begin{array}{cccccccc}
\gamma_{1}(0) & -\tau_{2}(a s+1) & 0 & 0 & 0 & 0 & \ldots & 0 \\
0 & \tau_{1}(1) & \gamma_{2}(a s) & 0 & 0 & 0 & \ldots & 0 \\
0 & 0 & \gamma_{1}(b) & -\tau_{2}(a[s-1]+1) & 0 & 0 & \ldots & 0 \\
0 & 0 & 0 & \tau_{1}(b+1) & \gamma_{2}(a[s-1]) & 0 & \ldots & 0 \\
\vdots & \vdots & & \ddots & \ddots & \ddots & \ddots & \vdots \\
0 & 0 & \ldots & & 0 & \gamma_{1}(b s) & -\tau_{2}(1) & 0 \\
0 & 0 & \ldots & & 0 & 0 & \tau_{1}(b s+1) & \gamma_{2}(0)
\end{array}\right)
$$


for the map at stage $2(s+1)$, and

$$
\left(\begin{array}{cccccccc}
\tau_{1}(0) & \tau_{2}(a s) & 0 & 0 & 0 & 0 & \cdots & 0 \\
0 & \gamma_{1}(1) & -\gamma_{2}(a[s-1]+1) & 0 & 0 & 0 & \cdots & 0 \\
0 & 0 & \tau_{1}(b) & \tau_{2}(a[s-1]) & 0 & 0 & \cdots & 0 \\
0 & 0 & 0 & \gamma_{1}(b+1) & -\gamma_{2}(a[s-2]+1) & 0 & \cdots & 0 \\
\vdots & \vdots & & \ddots & \ddots & \ddots & \ddots & \vdots \\
0 & 0 & \ldots & & 0 & \gamma_{1}(b[s-1]+1) & -\gamma_{2}(1) & 0 \\
0 & 0 & \ldots & & 0 & 0 & \tau_{1}(b s) & \tau_{2}(0)
\end{array}\right)
$$

for the map at stage $2 s+1$.

Now, for each $n \geq 0$, denote the generator $\epsilon(i, n-i)$ by $f_{i}^{n}$, so that the $n$-th bimodule in the total complex $\mathbb{P}$ is

$$
P_{n}=\bigoplus_{i=0}^{n} A^{\mathrm{e}} f_{i}^{n},
$$

the free $A^{\mathrm{e}}$-module of rank $n+1$ having generators

$$
\left\{f_{0}^{n}, f_{1}^{n}, \ldots, f_{n}^{n}\right\}
$$

Then the maps $P_{n} \stackrel{d_{n}}{\rightarrow} P_{n-1}$ in $\mathbb{P}$ are given by

$$
\begin{gathered}
d_{2 t}: f_{i}^{2 t} \mapsto \begin{cases}\gamma_{2}\left(\frac{a i}{2}\right) f_{i}^{2 t-1}+\gamma_{1}\left(\frac{2 b t-b i}{2}\right) f_{i-1}^{2 t-1}, & \text { for } i \text { even, } \\
-\tau_{2}\left(\frac{a i-a+2}{2}\right) f_{i}^{2 t-1}+\tau_{1}\left(\frac{2 b t-b i-b+2}{2}\right) f_{i-1}^{2 t-1}, & \text { for } i \text { odd, }\end{cases} \\
d_{2 t+1}: f_{i}^{2 t+1} \mapsto \begin{cases}\tau_{2}\left(\frac{a i}{2}\right) f_{i}^{2 t}+\gamma_{1}\left(\frac{2 b t-b i+2}{2}\right) f_{i-1}^{2 t}, & \text { for } i \text { even, } \\
-\gamma_{2}\left(\frac{a i-a+2}{2}\right) f_{i}^{2 t}+\tau_{1}\left(\frac{2 b t-b i+b}{2}\right) f_{i-1}^{2 t}, & \text { for } i \text { odd, }\end{cases}
\end{gathered}
$$

where we use the convention $f_{-1}^{n}=f_{n+1}^{n}=0$. The following result shows that the complex is exact.

Proposition 2.1. The complex $\mathbb{P}$ is exact, and is therefore a minimal projective resolution

$$
\mathbb{P}: \cdots \rightarrow P_{2} \stackrel{d_{2}}{\rightarrow} P_{1} \stackrel{d_{1}}{\rightarrow} P_{0} \stackrel{\mu}{\rightarrow} A \rightarrow 0
$$

of the left $A^{\mathrm{e}}$-module $A$.

Proof. We will show that the complex $\mathbb{P} \otimes_{A} k$ is exact, and a minimal projective resolution of the $A$-module $k$. Then the arguments in [Green and Snashall 2004] show that the complex $\mathbb{P}$ is exact. 
When applying $-\otimes_{A} k$ to $A^{\mathrm{e}}=A \otimes_{k} A^{\mathrm{op}}$, the elements $x$ and $y$ in $A^{\text {op }}$ become zero, and so the elements $\tau_{i}(s) \otimes 1$ and $\gamma_{i}(s) \otimes 1$ are just given by

$$
\begin{aligned}
& \tau_{1}(s) \otimes 1=-(x \otimes 1), \\
& \tau_{2}(s) \otimes 1=-q^{s}(y \otimes 1), \\
& \gamma_{1}(s) \otimes 1=\left(x^{a-1} \otimes 1\right), \\
& \gamma_{2}(s) \otimes 1=q^{(b-1) s}\left(y^{b-1} \otimes 1\right) .
\end{aligned}
$$

We shall identify these elements with $-x,-q^{s} y, x^{a-1}$ and $q^{(b-1) s} y^{b-1}$, respectively. Moreover, whenever the commutator element $q$ is involved, its precise power does not affect the dimensions of the vector spaces we are considering, so we shall write $q^{*}$ for simplicity.

Fix a number $n \geq 0$. The free bimodule $P_{n}$ has generators $\epsilon(i, j)$, with $n=i+j$ and $i, j \geq 0$. When the degree is not ambiguous, we shall denote the element

$$
\epsilon(i, j) \otimes 1 \in P_{n} \otimes_{A} k
$$

by $e_{j}$, and we shall denote the map

$$
P_{n} \otimes_{A} k \stackrel{d_{n} \otimes 1}{\longrightarrow} P_{n-1} \otimes_{A} k
$$

by $\widehat{d}_{n}$. Moreover, we denote by $U_{i}$ the left $A$-submodule of $P_{n-1} \otimes_{A} k$ generated by $\widehat{d}_{n}\left(e_{j}\right)$, so that

$$
\operatorname{Im} \widehat{d}_{n}=U_{0}+\cdots+U_{n} \subseteq P_{n-1} \otimes_{A} k .
$$

We now compute the dimensions of these modules $U_{i}$. Assume first that $n$ is even. Then

$$
\begin{aligned}
U_{0} & =A x^{a-1} e_{0}, \\
U_{i} & =A\left[\left(q^{*} y\right) e_{i-1}+\left(q^{*} x\right) e_{i}\right], \text { for odd } 0<i<n, \\
U_{i} & =A\left[\left(q^{*} y^{b-1}\right) e_{i-1}+\left(q^{*} x^{a-1}\right) e_{i}\right], \text { for even } 0<i<n, \\
U_{n} & =A y^{b-1} e_{n-1},
\end{aligned}
$$

and so we see that $\operatorname{dim} U_{0}=b, \operatorname{dim} U_{n}=a$, and otherwise $\operatorname{dim} U_{i}=a b-1$ and $\operatorname{dim} U_{j}=a+b+1$ for $i$ odd and $j$ even. When $n$ is odd, then

$$
\begin{aligned}
& U_{0}=A x e_{0}, \\
& U_{i}=A\left[\left(-q^{*} y\right) e_{i-1}+\left(q^{*} x^{a-1}\right) e_{i}\right], \text { for odd } 0<i<n, \\
& U_{i}=A\left[\left(q^{*} y^{b-1}\right) e_{i-1}+\left(q^{*} x\right) e_{i}\right], \text { for even } 0<i<n, \\
& U_{n}=A y e_{n-1},
\end{aligned}
$$


and so in this case we see that $\operatorname{dim} U_{0}=b(a-1), \operatorname{dim} U_{n}=a(b-1)$, and otherwise $\operatorname{dim} U_{i}=a(b-1)+1$ and $\operatorname{dim} U_{j}=b(a-1)+1$ for $i$ odd and $j$ even.

Our aim is to compute the dimensions of various intersections and sums obtained from the modules $U_{i}$. In order to do this, we need the fact that for any elements $z_{1}, z_{2} \in A$, the implication

$$
z_{1} x^{s}=z_{2} y^{t} \Longrightarrow z_{1}=v_{1} y^{t}+w_{1} x^{a-s} \text { and } z_{2}=v_{2} x^{s}+w_{2} y^{b-t}
$$

holds, where $v_{i}$ and $w_{i}$ are some elements in $A$ depending on $z_{1}$ and $z_{2}$. To see this, write

$$
z_{1}=g_{0}+g_{1} y+\cdots+g_{b-1} y^{b-1} \quad \text { and } \quad z_{2}=h_{0}+h_{1} y+\cdots+h_{b-1} y^{b-1},
$$

where the $g_{i}$ and $h_{i}$ are polynomials in $x$. Then

$$
\sum_{i} h_{i} y^{t+i}=z_{2} y^{t}=z_{1} x^{s}=\sum_{j}\left(q^{-j s} g_{j} x^{s}\right) y^{j},
$$

and comparing the coefficients of $y^{j}$, we find that $g_{j} x^{s}=0$ for $j<t$. Therefore, for these values of $j$, the polynomial $g_{j}$ must be a multiple of $x^{a-s}$. Then we can write

$$
\sum_{j<t} g_{j} y^{j}=w_{1} x^{a-s}
$$

for some $w_{1} \in A$, giving

$$
z_{1}=\sum_{j<t} g_{j} y^{j}+\sum_{j \geq t} g_{j} y^{j}=w_{1} x^{a-s}+v_{1} y^{t},
$$

where $v_{1}=\sum_{j \geq t} g_{j} y^{j-t}$. This proves the statement for $z_{1}$, and the proof for $z_{2}$ is similar.

We now compute the intersections of pairs of the modules $U_{i}$. Suppose $n$ is even, and fix an even integer $0 \leq j \leq n$. If $u$ belongs to $U_{j} \cap U_{j+1}$, then there are elements $z_{1}, z_{2} \in A$ such that

$$
u=z_{1}\left[\left(q^{*} y^{b-1}\right) e_{j-1}+\left(q^{*} x^{a-1}\right) e_{j}\right]=z_{2}\left[\left(q^{*} y\right) e_{j}+\left(q^{*} x\right) e_{j+1}\right] .
$$

The coefficients of $e_{j-1}$ and $e_{j+1}$ must be zero, whereas those of $e_{j}$ must be equal, giving

$$
\left(z_{1} q^{*}\right) x^{a-1}=\left(z_{2} q^{*}\right) y .
$$

By (2-1), there are elements $v_{1}, v_{2}, w_{1}, w_{2} \in A$ such that

$$
z_{1} q^{*}=v_{1} y+w_{1} x, \quad z_{2} q^{*}=v_{2} x^{a-1}+w_{2} y^{b-1},
$$

hence $u \in A y x^{a-1} e_{j}$. Conversely, any element in $A y x^{a-1} e_{j}$ belongs to $U_{j} \cap U_{j+1}$, showing

$$
U_{j} \cap U_{j+1}=A y x^{a-1} e_{j},
$$


and that the dimension of this intersection is $b-1$. Similarly, we compute three other types of intersections using the same method, and record everything in the table:

\begin{tabular}{c|cll}
$n$ & $j$ & intersection & dimension \\
\hline even & even & $U_{j} \cap U_{j+1}=A y x^{a-1} e_{j}$ & $b-1$ \\
even & odd & $U_{j} \cap U_{j+1}=A y^{b-1} x e_{j}$ & $a-1$ \\
odd & even & $U_{j} \cap U_{j+1}=A y x e_{j}$ & $(a-1)(b-1)$ \\
odd & odd & $U_{j} \cap U_{j+1}=A y^{b-1} x^{a-1} e_{j}$ & 1
\end{tabular}

Next we show that the equality

$$
\left(U_{0}+U_{1}+\cdots+U_{s}\right) \cap U_{s+1}=U_{s} \cap U_{s+1}
$$

holds for any $s \geq 1$. Suppose first that both $n$ and $s$ are even. The inclusion $U_{s} \cap U_{s+1} \subseteq\left(U_{0}+U_{1}+\cdots+U_{s}\right) \cap U_{s+1}$ obviously holds, so suppose $u$ is an element belonging to $\left(U_{0}+U_{1}+\cdots+U_{s}\right) \cap U_{s+1}$. Then $u$ can be written as

$$
\begin{aligned}
u & =z_{0} x^{a-1} e_{0}+z_{1}\left[\left(q^{*} y\right) e_{0}+\left(q^{*} x\right) e_{1}\right]+\cdots+z_{s}\left[\left(q^{*} y^{b-1}\right) e_{s-1}+\left(q^{*} x^{a-1}\right) e_{s}\right] \\
& =z_{s+1}\left[\left(q^{*} y\right) e_{s}+\left(q^{*} x\right) e_{s+1}\right],
\end{aligned}
$$

in which the coefficient of $e_{s+1}$ must be zero. Moreover, the coefficients of $e_{s}$ must be equal, that is,

$$
\left(z_{s+1} q^{*}\right) y=\left(z_{s} q^{*}\right) x^{a-1},
$$

and so from (2-1) we see that there exist elements $v, w \in A$ such that

$$
z_{s+1}=v x^{a-1}+w y^{b-1} .
$$

This gives

$$
u=\left(v x^{a-1}+w y^{b-1}\right) q^{*} y e_{s}=v q^{*} x^{a-1} y e_{s},
$$

and we see directly that $u$ belongs to $U_{s} \cap U_{s+1}$. Equation (2-2) therefore holds when $n$ and $s$ are even, and the same arguments show that the equality holds regardless of the parity of $n$ and $s$.

Using what we just showed, an induction argument gives the equality

$$
\operatorname{dim}\left(U_{0}+\cdots+U_{s}\right)=\sum_{i=0}^{s} \operatorname{dim} U_{i}-\sum_{i=0}^{s-1} \operatorname{dim}\left(U_{i} \cap U_{i+1}\right) .
$$

Then by counting dimensions, we see that the dimension of $\operatorname{Im} \widehat{d}_{n}$ is given by

$$
\operatorname{dim} \operatorname{Im} \widehat{d}_{n}= \begin{cases}t a b+1, & \text { when } n=2 t, \\ (t+1) a b-1, & \text { when } n=2 t+1 .\end{cases}
$$


The exactness of the complex $\mathbb{P} \otimes_{A} k$ now follows easily; the image of $\widehat{d}_{n+1}$ is contained in the kernel of $\widehat{d}_{n}$, and the dimension of $P_{n} \otimes_{A} k$ is $a b(n+1)$. It follows that $\operatorname{Im} \widehat{d}_{n+1}$ and $\operatorname{Ker} \widehat{d}_{n}$ are of the same dimension.

As for minimality, it suffices to show that $\operatorname{Im} \widehat{d}_{n}$ does not have a projective summand. This follows from the description of this module as the sum of $U_{i}$. Namely, we see directly that the element $y^{b-1} x^{a-1} \in A$ annihilates each $U_{i}$, and therefore also $\operatorname{Im} \widehat{d}_{n}$.

\section{Hochschild (co)homology}

Having obtained the bimodule resolution of $A=k\langle X, Y\rangle /\left(X^{a}, X Y-q Y X, Y^{b}\right)$, we turn now to its Hochschild homology and cohomology groups. Let $B$ be a bimodule, and recall that the Hochschild homology of $A$ with coefficients in $B$, denoted $\mathrm{HH}_{*}(A, B)$, is the $k$-vector space

$$
\mathrm{HH}_{*}(A, B)=\operatorname{Tor}_{*}^{A^{\mathrm{e}}}(B, A),
$$

where $B$ is viewed as a right $A^{\mathrm{e}}$-module. Dually, the Hochschild cohomology of $A$ with coefficients in $B$, denoted $\operatorname{HH}^{*}(A, B)$, is the $k$-vector space

$$
\operatorname{HH}^{*}(A, B)=\operatorname{Ext}_{A^{\mathrm{e}}}^{*}(A, B),
$$

where $B$ is viewed as a left $A^{\mathrm{e}}$-module. Of particular interest is the case $B=A$, namely the Hochschild homology and cohomology of $A$, denoted $\mathrm{HH}_{*}(A)$ and $\mathrm{HH}^{*}(A)$, respectively. Now, by viewing $A$ and $B$ as left $A^{\mathrm{e}}$-modules, it follows from [Cartan and Eilenberg 1956, VI.5.3] that $D\left(\mathrm{HH}^{*}(A, B)\right)$ is isomorphic, as a vector space, to $\operatorname{Tor}_{*}^{A^{\mathrm{e}}}(D(B), A)$, where $D$ denotes the usual $k$-dual $\operatorname{Hom}_{k}(-, k)$. In particular, by taking $B=A$, we see that

$$
\operatorname{dim}_{k} \operatorname{HH}^{n}(A)=\operatorname{dim}_{k} \operatorname{Tor}_{n}^{A^{\mathrm{e}}}(D(A), A)
$$

for all $n \geq 0$.

Our algebra $A$ is Frobenius; it is easy to check that the map $A \stackrel{\phi}{\rightarrow} D(A)$ of left $A$-modules, defined by

$$
\phi(1): \sum_{\substack{0 \leq j \leq b-1 \\ 0 \leq i \leq a-1}} c_{j, i} y^{j} x^{i} \mapsto c_{b-1, a-1},
$$

is an isomorphism. To such a Frobenius isomorphism, one can always associate a $k$-algebra automorphism $A \stackrel{\nu}{\rightarrow} A$, a Nakayama automorphism, with the (defining) property that

$$
w \cdot \phi(1)=\phi(1) \cdot v(w)
$$


for all elements $w \in A$. In our case, the elements $x$ and $y$ generate $A$, and since

$$
x \cdot \phi(1)=\phi(1) \cdot q^{1-b} x \quad \text { and } \quad y \cdot \phi(1)=\phi(1) \cdot q^{a-1} y,
$$

we see that the automorphism defined by

$$
v: x \mapsto q^{1-b} x, y \mapsto q^{a-1} y
$$

is a Nakayama automorphism. The composite map $\phi \circ v^{-1}$ is then a bimodule isomorphism between the right $A^{\mathrm{e}}$-modules ${ }_{v} A_{1}$ and $D(A)$, where the scalar action on ${ }_{v} A_{1}$ is given by

$$
u \cdot\left(w_{1} \otimes w_{2}\right)=v\left(w_{2}\right) u w_{1} .
$$

Consequently, we see that

$$
\operatorname{dim}_{k} \operatorname{HH}^{n}(A)=\operatorname{dim}_{k} \operatorname{Tor}_{n}^{A^{\mathrm{e}}}\left({ }_{\nu} A_{1}, A\right)
$$

for all $n \geq 0$.

Now let $\alpha, \beta \in k$ be nonzero scalars, and let $A \stackrel{\psi}{\rightarrow} A$ be the automorphism defined by $x \mapsto \alpha x$ and $y \mapsto \beta y$. Tensoring the deleted projective bimodule resolution $\mathbb{P}_{A}$ with the right $A^{\mathrm{e}}$-module ${ }_{\psi} A_{1}$, we obtain an isomorphism

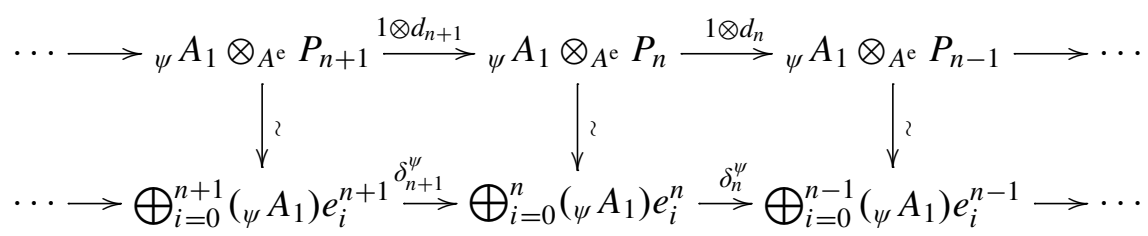

of complexes, where $\left\{e_{0}^{n}, e_{1}^{n}, \ldots, e_{n}^{n}\right\}$ is the standard generating set of $n+1$ copies of ${ }_{\psi} A_{1}$. The map $\delta_{n}^{\psi}$ is then given by

$\delta_{2 t}^{\psi}: y^{u} x^{v} e_{i}^{2 t} \mapsto$

$$
\left\{\begin{aligned}
K_{1}^{\psi}(t, i, u, v) y^{u+b-1} x^{v} e_{i}^{2 t-1}+K_{2}^{\psi}(t, i, u, v) y^{u} x^{v+a-1} e_{i-1}^{2 t-1}, & \text { for } i \text { even, } \\
{\left[q^{(a i-a+2+2 v) / 2}-\beta\right] y^{u+1} x^{v} e_{i}^{2 t-1} } & \\
+\left[\alpha q^{(2 b t-b i-b+2+2 u) / 2}-1\right] y^{u} x^{v+1} e_{i-1}^{2 t-1}, & \text { for } i \text { odd }
\end{aligned}\right.
$$

$\delta_{2 t+1}^{\psi}: y^{u} x^{v} e_{i}^{2 t+1} \mapsto$

$$
\begin{cases}{\left[\beta-q^{(a i+2 v) / 2}\right] y^{u+1} x^{v} e_{i}^{2 t}+K_{3}^{\psi}(t, i, u, v) y^{u} x^{v+a-1} e_{i-1}^{2 t},} & \text { for } i \text { even, } \\ K_{4}^{\psi}(t, i, u, v) y^{u+b-1} x^{v} e_{i}^{2 t}+\left[\alpha q^{(2 b t-b i+b+2 u) / 2}-1\right] y^{u} x^{v+1} e_{i-1}^{2 t}, & \text { for } i \text { odd, }\end{cases}
$$

where we use the convention $e_{-1}^{n}=e_{n+1}^{n}=0$. Here the elements $K_{j}^{\psi}(t, i, u, v)$, which are scalars whose values depend on the parameters $\psi, t, i, u$ and $v$, are 
defined as follows:

$$
\begin{aligned}
& K_{1}^{\psi}(t, i, u, v)=\sum_{j=0}^{b-1} q^{j(a i+2 v) / 2} \beta^{b-1-j}, \\
& K_{2}^{\psi}(t, i, u, v)=\sum_{j=0}^{a-1} q^{j(2 b t-b i+2 u) / 2} \alpha^{j}, \\
& K_{3}^{\psi}(t, i, u, v)=\sum_{j=0}^{a-1} q^{j(2 b t-b i+2+2 u) / 2} \alpha^{j}, \\
& K_{4}^{\psi}(t, i, u, v)=\sum_{j=0}^{b-1} q^{j(a i-a+2+2 v) / 2} \beta^{b-1-j} .
\end{aligned}
$$

When $q$ is not a root of unity, and the characteristic of $k$ does not divide $a$ or $b$, these scalars are all nonzero when the automorphism $\psi$ is either the identity or the Nakayama automorphism. For, in this case, the elements are of the form

$$
q^{s}\left(1+q^{m}+q^{2 m}+\cdots+q^{r m}\right)
$$

for some $m, s \in \mathbb{Z}$ and $r=a-1$ or $r=b-1$. When $m=0$, this element is nonzero since the characteristic of $k$ does not divide $a$ or $b$, and, if it was zero for some $m \neq 0$, then $q$ would be a root of unity because of the equality

$$
\left(1+q^{m}+q^{2 m}+\cdots+q^{r m}\right)\left(1-q^{m}\right)=1-q^{(r+1) m} .
$$

In the following result we use this complex to compute the Hochschild homology of our algebra $A$.

Theorem 3.1. When $q$ is not a root of unity, the Hochschild homology of A is given by

$$
\operatorname{dim}_{k} \operatorname{HH}_{n}(A)=\left\{\begin{array}{l}
a+b-1, \text { when } n=0, \\
a+b, \quad \text { when } n \geq 1 \text { and char } k \text { divides both } a \text { and } b, \\
a+b-1, \text { when } n \geq 1 \text { and char } k \text { divides one of } a \text { and } b, \\
a+b-2, \text { when } n \geq 1 \text { and char } k \text { does not divide } a \text { or } b .
\end{array}\right.
$$

Proof. We need to compute the homology groups of the above complex in the case when $\psi$ is the identity automorphism on $A$, that is, when $\alpha=1=\beta$. We do this by computing $\operatorname{Ker} \delta_{2 t}^{1}$ for $t \geq 1$ and $\operatorname{Ker} \delta_{2 t+1}^{1}$ for $t \geq 0$, and we treat these two cases separately.

$\operatorname{Ker} \delta_{2 t}^{\mathbf{1}}$. The image under the map $\delta_{2 t}^{1}$ of a basis vector

$$
y^{u} x^{v} e_{i}^{2 t} \in \bigoplus_{i=0}^{2 t} A e_{i}^{2 t}
$$


is given by

$$
\left\{\begin{aligned}
K_{1}^{1}(t, i, u, v) y^{u+b-1} x^{v} e_{i}^{2 t-1}+K_{2}^{1}(t, i, u, v) y^{u} x^{v+a-1} e_{i-1}^{2 t-1}, & \text { for } i \text { even, } \\
{\left[q^{(a i-a+2+2 v) / 2}-1\right] y^{u+1} x^{v} e_{i}^{2 t-1} } & \\
+\left[q^{(2 b t-b i-b+2+2 u) / 2}-1\right] y^{u} x^{v+1} e_{i-1}^{2 t-1}, & \text { for } i \text { odd. }
\end{aligned}\right.
$$

From the definition of the scalars $K_{1}^{1}$ and $K_{2}^{1}$, we see that

$$
\begin{aligned}
& K_{1}^{1}(t, i, u, v)=0 \Longleftrightarrow i=0, v=0, \operatorname{char} k \mid b, \\
& K_{2}^{1}(t, i, u, v)=0 \Longleftrightarrow i=2 t, u=0, \operatorname{char} k \mid a,
\end{aligned}
$$

and therefore we first compute the dimension of $\operatorname{Ker} \delta_{2 t}^{1}$ under the assumption that the characteristic of $k$ does not divide $a$ or $b$.

First, we count the number of single basis vectors in $\bigoplus_{i=0}^{2 t} A e_{i}^{2 t}$ belonging to $\operatorname{Ker} \delta_{2 t}^{1}$. For even $i$, we have

$$
\begin{aligned}
\delta_{2 t}^{1}\left(y^{u} x^{v} e_{i}^{2 t}\right)=0 \text { for all even } i & \Longleftrightarrow u+b-1 \geq b \text { and } v+a-1 \geq a \\
& \Longleftrightarrow 1 \leq u \leq b-1 \text { and } 1 \leq v \leq a-1,
\end{aligned}
$$

from which we obtain $(b-1)(a-1)(t+1)$ vectors (there are $t+1$ even numbers in the set $\{0,1, \ldots, 2 t\})$. For odd $i$, we have

$$
\begin{aligned}
\delta_{2 t}^{1}\left(y^{u} x^{v} e_{i}^{2 t}\right)=0 \text { for all odd } i & \Longleftrightarrow u+1 \geq b \text { and } v+1 \geq a \\
& \Longleftrightarrow u=b-1 \text { and } v=a-1,
\end{aligned}
$$

giving $t$ vectors (there are $t$ odd numbers in the set $\{0,1, \ldots, 2 t\}$ ). Next, we count the other single basis vectors which are mapped to zero, starting with those for which $i$ is even. The element $e_{2 t}^{2 t-1}$ is zero by definition. Hence when $i=2 t$ and $v+a-1 \geq a$, that is, when $1 \leq v \leq a-1$, we see that $y^{u} x^{v} e_{i}^{2 t}$ maps to zero. But the vectors for which $u$ is nonzero were counted above. Hence the new vectors are $x^{v} e_{2 t}^{2 t}$ for $1 \leq v \leq a-1$. Similarly, the element $e_{-1}^{2 t-1}$ is zero by definition. Hence when $i=0$ and $u+b-1 \geq b$, that is, when $1 \leq u \leq b-1$, we see that $y^{u} x^{v} e_{i}^{2 t}$ maps to zero. But here the vectors for which $v$ is nonzero were counted above, and so the new vectors are $y^{u} e_{0}^{2 t}$ for $1 \leq u \leq b-1$. It is easy to see that except for these $a+b-2$ new vectors, there is no other single basis vector $y^{u} x^{v} e_{i}^{2 t}$ in $\operatorname{Ker} \delta_{2 t}^{1}$ for which $i$ is even, since both $K_{1}^{1}(t, i, u, v)$ and $K_{2}^{1}(t, i, u, v)$ are always nonzero. Moreover, when $i$ is odd, neither $e_{i}^{2 t-1}$ nor $e_{i-1}^{2 t-1}$ are zero, and the coefficients

$$
\left[q^{(a i-a+2+2 v) / 2}-1\right], \quad \text { and }\left[q^{(2 b t-b i-b+2+2 u) / 2}-1\right]
$$

are both nonzero. Hence in this case there are no new basis vectors mapped to zero. 
Now we count the number of nontrivial linear combinations of two or more basis vectors in $\bigoplus_{i=0}^{2 t} A e_{i}^{2 t}$ belonging to Ker $\delta_{2 t}^{1}$. Let $i$ be even. If the first term of $\delta_{2 t}^{1}\left(y^{u} x^{v} e_{i}^{2 t}\right)$ is nonzero, then the only way to "kill" it is to involve the second term of $\delta_{2 t}^{1}\left(y^{u+b-1} x^{v-1} e_{i+1}^{2 t}\right)$. Thus to get a nontrivial linear combination, we see that $u, v$ and $i$ must satisfy $u=0,1 \leq v \leq a-1$ and $i=0,2, \ldots, 2 t-2$. For these parameter values, the second term of $\delta_{2 t}^{1}\left(y^{u} x^{v} e_{i}^{2 t}\right)$ vanishes, as does the first term of $\delta_{2 t}^{1}\left(y^{u+b-1} x^{v-1} e_{i+1}^{2 t}\right)$. Therefore, for a suitable nonzero scalar $C(a, b, i, u, v)$, the linear combination

$$
x^{v} e_{i}^{2 t}+C(a, b, i, u, v) y^{b-1} x^{v-1} e_{i+1}^{2 t}
$$

is mapped to zero for $1 \leq v \leq a-1$ and $i=0,2, \ldots, 2 t-2$, and there are $(a-1) t$ such elements. If the second term of $\delta_{2 t}^{1}\left(y^{u} x^{v} e_{i}^{2 t}\right)$ is nonzero, then the only way to "kill" it is to involve the first term of $\delta_{2 t}^{1}\left(y^{u-1} x^{v+a-1} e_{i-1}^{2 t}\right)$. To get a nontrivial linear combination, the parameters $u, v$ and $i$ must satisfy $1 \leq u \leq b-1, v=0$ and $i=2,4, \ldots, 2 t$, and for these values the first term of $\delta_{2 t}^{1}\left(y^{u} x^{v} e_{i}^{2 t}\right)$ and the second term of $\delta_{2 t}^{1}\left(y^{u-1} x^{v+a-1} e_{i-1}^{2 t}\right)$ vanish. Thus, for a suitable nonzero scalar $C^{\prime}(a, b, i, u, v)$, the linear combination

$$
y^{u} e_{i}^{2 t}+C^{\prime}(a, b, i, u, v) y^{u-1} x^{a-1} e_{i-1}^{2 t}
$$

is mapped to zero for $1 \leq u \leq b-1$ and $i=2,4, \ldots, 2 t$, and there are $(b-1) t$ such elements.

We have now accounted for all the elements of $\operatorname{Ker} \delta_{2 t}^{1}$, when the characteristic of $k$ does not divide $a$ or $b$. If the characteristic of $k$ divides $a$, then we must add to our list the element $e_{2 t}^{2 t}$. Similarly, if the characteristic of $k$ divides $b$, then we must add to our list the element $e_{0}^{2 t}$. Finally, if the characteristic of $k$ divides both $a$ and $b$, then we must add both these two elements to our list (and they are different elements since $t \geq 1$ ). Summing up, we see that the total dimension of $\operatorname{Ker} \delta_{2 t}^{1}$ is given by

$$
\operatorname{dim}_{k} \operatorname{Ker} \delta_{2 t}^{1}= \begin{cases}a b t+a b-1, & \text { when char } k \text { does not divide } a \text { or } b \\ a b t+a b+1, & \text { when char } k \text { divides both } a \text { and } b \\ a b t+a b, & \text { otherwise. }\end{cases}
$$

$\operatorname{Ker} \delta_{2 t+1}^{1}$. The image under the map $\delta_{2 t+1}^{1}$ of a basis vector

$$
y^{u} x^{v} e_{i}^{2 t+1} \in \bigoplus_{i=0}^{2 t+1} A e_{i}^{2 t+1}
$$


is given by

$$
\begin{cases}{\left[1-q^{(a i+2 v) / 2}\right] y^{u+1} x^{v} e_{i}^{2 t}+K_{3}^{1}(t, i, u, v) y^{u} x^{v+a-1} e_{i-1}^{2 t},} & \text { for } i \text { even } \\ K_{4}^{1}(t, i, u, v) y^{u+b-1} x^{v} e_{i}^{2 t}+\left[q^{(2 b t-b i+b+2 u) / 2}-1\right] y^{u} x^{v+1} e_{i-1}^{2 t}, & \text { for } i \text { odd }\end{cases}
$$

From the definition of the elements $K_{3}^{1}$ and $K_{4}^{1}$, we see that they are always nonzero, contrary to the case above where there were parameters for which $K_{1}^{1}$ and $K_{2}^{1}$ vanished. Therefore, the characteristic of $k$ does not matter when we compute the dimension of $\operatorname{Ker} \delta_{2 t+1}^{1}$.

We follow the same procedure as we did for $\operatorname{Ker} \delta_{2 t}^{1}$. First we count the number of single basis vectors in $\bigoplus_{i=0}^{2 t+1} A e_{i}^{2 t+1}$ belonging to $\operatorname{Ker} \delta_{2 t+1}^{1}$. For even $i$, we have

$$
\begin{aligned}
\delta_{2 t+1}^{1}\left(y^{u} x^{v} e_{i}^{2 t+1}\right)=0 \text { for all even } i & \Longleftrightarrow u+1 \geq b \text { and } v+a-1 \geq a \\
& \Longleftrightarrow u=b-1 \text { and } 1 \leq v \leq a-1,
\end{aligned}
$$

resulting in $(a-1)(t+1)$ vectors (there are $(t+1)$ even numbers in the set $\{0,1, \ldots, 2 t+1\})$. When $i$ is odd, we have

$$
\begin{aligned}
\delta_{2 t+1}^{1}\left(y^{u} x^{v} e_{i}^{2 t+1}\right)=0 \text { for all odd } i & \Longleftrightarrow u+b-1 \geq b \text { and } v+1 \geq a \\
& \Longleftrightarrow 1 \leq u \leq b-1 \text { and } v=a-1,
\end{aligned}
$$

giving $(b-1)(t+1)$ vectors (there are $(t+1)$ odd numbers in the set $\{0,1, \ldots, 2 t+$ $1\})$. Next, we count the other single basis vectors in $\bigoplus_{i=0}^{2 t+1} A e_{i}^{2 t+1}$ belonging to $\operatorname{Ker} \delta_{2 t+1}^{1}$, starting with those for which $i$ is even. The element $e_{-1}^{2 t}$ is zero; hence for $i=0$ the second term in $\delta_{2 t+1}^{1}\left(y^{u} x^{v} e_{i}^{2 t+1}\right)$ vanishes. If now $v=0$, then the coefficient $\left[1-q^{(a i+2 v) / 2}\right]$ vanishes, and therefore the vector $y^{u} e_{0}^{2 t+1}$ maps to zero for $0 \leq u \leq b-1$. There are $b$ such vectors, and none of them was counted above. Moreover, it is not hard to see that there is no other vector $y^{u} x^{v} e_{i}^{2 t+1}$ in $\operatorname{Ker} \delta_{2 t+1}^{1}$ for which $i$ is even. As for the case when $i$ is odd, the element $e_{2 t+1}^{2 t}$ is zero by definition, and the coefficient $\left[q^{(2 b t-b i+b+2 u) / 2}-1\right]$ vanishes for $i=2 t+1$ and $u=0$. Therefore, the vector $x^{v} e_{2 t+1}^{2 t+1}$ maps to zero for $0 \leq v \leq a-1$. These $a$ vectors have not been counted before, and $\operatorname{Ker} \delta_{2 t+1}^{1}$ does not contain more vectors $y^{u} x^{v} e_{i}^{2 t+1}$ for which $i$ is odd.

At last we count the number of nontrivial linear combinations of two or more basis vectors in $\bigoplus_{i=0}^{2 t+1} A e_{i}^{2 t+1}$ belonging to Ker $\delta_{2 t+1}^{1}$. Let $i$ be even, and suppose the first term of $\delta_{2 t+1}^{1}\left(y^{u} x^{v} e_{i}^{2 t+1}\right)$ is nonzero. The only way to cancel this term is to involve the second term of $\delta_{2 t+1}^{1}\left(y^{u+1} x^{v-1} e_{i+1}^{2 t+1}\right)$. Now, the first term in the latter vanishes, as does the second term of $\delta_{2 t+1}^{1}\left(y^{u} x^{v} e_{i}^{2 t+1}\right)$, since $v$ must be nonzero. Thus, for a suitable nonzero scalar $C^{\prime \prime}(a, b, i, u, v)$, the element

$$
y^{u} x^{v} e_{i}^{2 t+1}+C^{\prime \prime}(a, b, i, u, v) y^{u+1} x^{v-1} e_{i+1}^{2 t+1}
$$


belongs to $\operatorname{Ker} \delta_{2 t+1}^{1}$, when the parameters satisfy $0 \leq u \leq b-2,1 \leq v \leq a-1$ and $i=0,2, \ldots, 2 t$. There are $(a-1)(b-1)(t+1)$ such elements. Finally, suppose the second term of $\delta_{2 t+1}^{1}\left(y^{u} x^{v} e_{i}^{2 t+1}\right)$ is nonzero. To cancel it, we must involve the first term in $\delta_{2 t+1}^{1}\left(y^{u-b+1} x^{v+a-1} e_{i-1}^{2 t+1}\right)$, and so we see that the only possibility for $u$ and $v$ is $u=b-1$ and $v=0$. Therefore, for a suitable nonzero scalar $C^{\prime \prime \prime}(a, b, i, u, v)$, the element

$$
y^{b-1} e_{i}^{2 t+1}+C^{\prime \prime \prime}(a, b, i, u, v) x^{a-1} e_{i-1}^{2 t+1}
$$

is mapped to zero for $i=2,4, \ldots, 2 t$. There are $t$ such linear combinations.

All the elements of $\operatorname{Ker} \delta_{2 t+1}^{1}$ are now accounted for, and so when summing up we obtain the dimension of this vector space:

$$
\operatorname{dim}_{k} \operatorname{Ker} \delta_{2 t+1}^{1}=a b t+a b+a+b-1 .
$$

Using the identities

$$
\operatorname{dim}_{k} \operatorname{Ker} \delta_{n}^{1}+\operatorname{dim}_{k} \operatorname{Im} \delta_{n}^{1}=\operatorname{dim}_{k} A^{n+1}=(n+1) a b,
$$

we can now calculate the Hochschild homology of $A$. The dimension formula gives $\operatorname{dim}_{k} \operatorname{Im} \delta_{2 t+1}^{1}=a b t+a b-a-b+1$, in particular $\operatorname{dim}_{k} \operatorname{Im} \delta_{1}^{1}=2 a b-a-b+1$, giving

$$
\operatorname{dim}_{k} \mathrm{HH}_{0}(A)=\operatorname{dim}_{k} A-\operatorname{dim}_{k} \operatorname{Im} \delta_{1}^{1}=a+b-1 .
$$

Applying the formula to the results we obtained, when computing $\operatorname{Ker} \delta_{2 t}^{1}$,

$$
\operatorname{dim}_{k} \operatorname{Im} \delta_{2 t+2}^{1}= \begin{cases}a b t+a b+1, & \text { when char } k \text { does not divide } a \text { or } b, \\ a b t+a b-1, & \text { when char } k \text { divides both } a \text { and } b, \\ a b t+a b, & \text { otherwise, }\end{cases}
$$

and so by calculating $\operatorname{dim}_{k} \operatorname{HH}_{n}(A)=\operatorname{dim}_{k} \operatorname{Ker} \delta_{n}^{1}-\operatorname{dim}_{k} \operatorname{Im} \delta_{n+1}^{1}$ for $n \geq 1$, we get

$$
\operatorname{dim}_{k} \operatorname{HH}_{n}(A)= \begin{cases}a+b-2, & \text { when char } k \text { does not divide } a \text { or } b \\ a+b, & \text { when char } k \text { divides both } a \text { and } b \\ a+b-1, & \text { otherwise. }\end{cases}
$$

This completes the proof.

In particular, since $a$ and $b$ are both at least 2, the Hochschild homology of $A$ does not vanish in high degrees (or in any degree). This was conjectured by Han [2006] to hold for all finite-dimensional algebras of infinite global dimension, and in the same paper it was proved that this conjecture holds for monomial algebras.

The converse of this conjecture always holds when the algebra modulo its radical is separable over the ground field. Namely, in this situation, if the global dimension of the algebra is finite, then the algebra has finite projective dimension as a bimodule, and hence its Hochschild homology vanishes in high degrees. The same holds 
of course for Hochschild cohomology, and following this easy observation, Happel [1989] remarked that "the converse seems to be not known". Thus the cohomology version of Han's conjecture came to be known as "Happel's question". However, this cohomology version is false in general; it was proved in [Buchweitz et al. 2005] that there do exist finite-dimensional algebras of infinite global dimension for which Hochschild cohomology vanishes in high degrees. The counterexample used in the paper was precisely our algebra $A$ with $a=2=b$, and the following result shows that the same holds for arbitrary $a$ and $b$. Contrary to the homology case, the dimensions of the cohomology groups do not depend on the characteristic of $k$.

Theorem 3.2. When $q$ is not a root of unity, the Hochschild cohomology of $A$ is given by

$$
\operatorname{dim}_{k} \operatorname{HH}^{n}(A)=\left\{\begin{array}{l}
2, \text { for } n=0 \\
2, \text { for } n=1 \\
1, \text { for } n=2 \\
0, \text { for } n \geq 3
\end{array}\right.
$$

In particular, the Hochschild cohomology of A vanishes in high degrees.

Proof. It is well known and easy to see that, in general, $\mathrm{HH}^{0}(A)$ is isomorphic to the center of $A$, that is, the subalgebra

$$
\{w \in A \mid w z=z w \text { for all } z \in A\} .
$$

The center of our algebra $A$ is the vector space spanned by the "first" and the "last" elements in its basis, namely the elements 1 and $y^{b-1} x^{a-1}$. Hence $\operatorname{HH}^{0}(A)$ is 2-dimensional.

To compute the Hochschild cohomology groups of positive degree, we compute the homology of the complex obtained prior to Theorem 3.1, in the case when $\psi$ is the Nakayama automorphism $\nu$. In this case, the scalars $\alpha$ and $\beta$ are given by

$$
\alpha=q^{1-b}, \quad \text { and } \quad \beta=q^{a-1} .
$$

We apply the same method as we did when computing homology; we compute $\operatorname{Ker} \delta_{2 t}^{v}$ for $t \geq 1$ and $\operatorname{Ker} \delta_{2 t+1}^{\nu}$ for $t \geq 0$, treating the two cases separately.

$\operatorname{Ker} \delta_{2 t^{*}}^{v}$. The result when applying the map $\delta_{2 t}^{v}$ to a basis vector

$$
y^{u} x^{v} e_{i}^{2 t} \in \bigoplus_{i=0}^{2 t}\left({ }_{v} A_{1}\right) e_{i}^{2 t}
$$


is given by

$$
\left\{\begin{array}{r}
K_{1}^{v}(t, i, u, v) y^{u+b-1} x^{v} e_{i}^{2 t-1}+K_{2}^{v}(t, i, u, v) y^{u} x^{v+a-1} e_{i-1}^{2 t-1}, \\
{\left[q^{(a i-a+2+2 v) / 2}-q^{a-1}\right] y^{u+1} x^{v} e_{i}^{2 t-1}} \\
+\left[q^{(2 b t-b i-3 b+4+2 u) / 2}-1\right] y^{u} x^{v+1} e_{i-1}^{2 t-1},
\end{array}\right.
$$

From the definition of the elements $K_{1}^{v}$ and $K_{2}^{v}$, we see that

$$
\begin{aligned}
& K_{1}^{v}(t, i, u, v)=0 \Longleftrightarrow i=0, v=a-1, \operatorname{char} k \mid b, \\
& K_{2}^{v}(t, i, u, v)=0 \Longleftrightarrow i=2 t, u=b-1, \operatorname{char} k \mid a,
\end{aligned}
$$

and so we first compute the dimension of $\operatorname{Ker} \delta_{2 t}^{\nu}$ in the case when the characteristic of $k$ does not divide $a$ or $b$.

First, we count the number of single basis vectors in $\left.\bigoplus_{i=0}^{2 t}{ }_{\nu} A_{1}\right) e_{i}^{2 t}$ belonging to $\operatorname{Ker} \delta_{2 t}^{\nu}$. As in the homology case, we have

$$
\begin{aligned}
\delta_{2 t}^{v}\left(y^{u} x^{v} e_{i}^{2 t}\right)=0 \text { for all even } i & \Longleftrightarrow u+b-1 \geq b \text { and } v+a-1 \geq a \\
& \Longleftrightarrow 1 \leq u \leq b-1 \text { and } 1 \leq v \leq a-1, \\
\delta_{2 t}^{v}\left(y^{u} x^{v} e_{i}^{2 t}\right)=0 \text { for all odd } i & \Longleftrightarrow u+1 \geq b \text { and } v+1 \geq a \\
& \Longleftrightarrow u=b-1 \text { and } v=a-1,
\end{aligned}
$$

from which we obtain $(b-1)(a-1)(t+1)+t$ vectors. Next, we count the other single basis vectors in $\bigoplus_{i=0}^{2 t}\left({ }_{v} A_{1}\right) e_{i}^{2 t}$ belonging to $\operatorname{Ker} \delta_{2 t}^{v}$. Since $K_{1}^{v}$ and $K_{2}^{v}$ are always nonzero, the number of such vectors for which $i$ is even is the same as in the homology case, namely $a+b-2$. As for the vectors for which $i$ is odd, it is no longer true that the coefficients are always nonzero. The coefficient $\left[q^{(a i-a+2+2 v) / 2}-q^{a-1}\right]$ vanishes when $i=1$ and $v=a-2$, whereas $\left[q^{(2 b t-b i-3 b+4+2 u) / 2}-1\right]$ vanishes when $i=2 t-1$ and $u=b-2$. Both these cases will occur, since $t$ is at least 1 when we compute $\operatorname{Ker} \delta_{2 t}^{v}$. However, these coefficients need to vanish simultaneously for the basis vector to belong to $\operatorname{Ker} \delta_{2 t}^{v}$, and this only happens when $t=1$, since then $2 t-1=1$. Thus, when $t=1$ the vector $y^{b-2} x^{a-2} e_{1}^{2}$ maps to zero, whereas when $t \geq 2$ there are no new basis vectors in $\operatorname{Ker} \delta_{2 t}^{v}$ for which $i$ is odd.

Now we count the number of nontrivial linear combinations of two or more basis vectors in $\bigoplus_{i=0}^{2 t}\left({ }_{v} A_{1}\right) e_{i}^{2 t}$ belonging to $\operatorname{Ker} \delta_{2 t}^{v}$. These elements are precisely the same as in the homology case, and we do not encounter problems because of the "new" basis vector in $\operatorname{Ker} \delta_{2}^{v}$ we obtained above. Therefore, the number of such linear combinations is $(a-1) t+(b-1) t$.

We now look at what happens when the characteristic of $k$ divides $a$ or $b$. If char $k$ divides $a$, then we must add the vector $x^{a-1} e_{0}^{2 t}$ to the list of single basis 
vectors mapped to zero. However, this vector already appears in one of the nontrivial linear combinations; hence it does not contribute to the total dimension. Similarly, when char $k$ divides $b$, then the new vector $y^{b-1} e_{2 t}^{2 t}$ belongs to the list of single basis vectors mapped to zero. But again this vector already appears in one of the nontrivial linear combinations, and it will therefore not contribute to the total dimension. This argument is still valid if char $k$ divides both $a$ and $b$. This shows that the dimension of $\operatorname{Ker} \delta_{2 t}^{\nu}$ is independent of the characteristic of $k$.

In total, we see that the dimension of $\operatorname{Ker} \delta_{2 t}^{v}$ is almost the same as it was in the homology case when the characteristic of $k$ did not divide $a$ or $b$; we need one additional vector when $t=1$. Therefore, the dimension is given by

$$
\operatorname{dim}_{k} \operatorname{Ker} \delta_{2 t}^{v}= \begin{cases}2 a b, & \text { when } t=1, \\ a b t+a b-1, & \text { when } t \geq 2 .\end{cases}
$$

$\operatorname{Ker} \delta_{2 t+1}^{v}$. The image under the map $\delta_{2 t+1}^{v}$ of a basis vector

$$
y^{u} x^{v} e_{i}^{2 t+1} \in \bigoplus_{i=0}^{2 t+1}\left({ }_{v} A_{1}\right) e_{i}^{2 t+1}
$$

is given by

$$
\begin{cases}{\left[q^{a-1}-q^{(a i+2 v) / 2}\right] y^{u+1} x^{v} e_{i}^{2 t}+K_{3}^{v}(t, i, u, v) y^{u} x^{v+a-1} e_{i-1}^{2 t},} & \text { for } i \text { even, } \\ K_{4}^{v}(t, i, u, v) y^{u+b-1} x^{v} e_{i}^{2 t}+\left[q^{(2 b t-b i-b+2+2 u) / 2}-1\right] y^{u} x^{v+1} e_{i-1}^{2 t}, & \text { for } i \text { odd. }\end{cases}
$$

Now, from the definition of the scalars $K_{3}^{v}$ and $K_{4}^{v}$, we see that $K_{3}^{v}$ is always nonzero, while we have

$$
K_{4}^{v}(t, i, u, v)=0 \Longleftrightarrow i=1, v=a-2, \operatorname{char} k \mid b .
$$

Therefore, we first compute the dimension of $\operatorname{Ker} \delta_{2 t+1}^{v}$ under the assumption that the characteristic of $k$ does not divide $b$.

First, we count the number of single basis vectors in $\bigoplus_{i=0}^{2 t+1}\left({ }_{v} A_{1}\right) e_{i}^{2 t+1}$ belonging to $\operatorname{Ker} \delta_{2 t+1}^{v}$. As in the homology case, we have

$$
\begin{aligned}
\delta_{2 t+1}^{v}\left(y^{u} x^{v} e_{i}^{2 t+1}\right)=0 \text { for all even } i & \Longleftrightarrow u+1 \geq b \text { and } v+a-1 \geq a \\
& \Longleftrightarrow u=b-1 \text { and } 1 \leq v \leq a-1, \\
\delta_{2 t+1}^{v}\left(y^{u} x^{v} e_{i}^{2 t+1}\right)=0 \text { for all odd } i & \Longleftrightarrow u+b-1 \geq b \text { and } v+1 \geq a \\
& \Longleftrightarrow 1 \leq u \leq b-1 \text { and } v=a-1,
\end{aligned}
$$

from which we obtain $(a-1)(t+1)+(b-1)(t+1)$ vectors. Next, we count the other single basis vectors in $\bigoplus_{i=0}^{2 t+1}\left({ }_{\nu} A_{1}\right) e_{i}^{2 t+1}$ belonging to $\operatorname{Ker} \delta_{2 t+1}^{\nu}$, treating first the ones for which $i$ is even. When $i=0$, the second term of $\delta_{2 t+1}^{v}\left(y^{u} x^{v} e_{i}^{2 t+1}\right)$ vanishes, and the first term then vanishes if $u=b-1$ or $v=a-1$. Some of 
these vectors are among the ones counted above, the new ones are $y^{b-1} e_{0}^{2 t+1}$ and $y^{u} x^{a-1} e_{0}^{2 t+1}$ for $0 \leq u \leq b-2$. Except for these $b$ elements, there are no other single basis elements in $\operatorname{Ker} \delta_{2 t+1}^{\nu}$ for which $i$ is even. As for those for which $i$ is odd, we see that the first term of $\delta_{2 t+1}^{v}\left(y^{u} x^{v} e_{i}^{2 t+1}\right)$ vanishes when $i=2 t+1$. In this case, the second term vanishes if $u=b-1$ or $v=a-1$, and of these vectors the ones which have not been counted before are the $a$ elements $x^{a-1} e_{2 t+1}^{2 t+1}$ and $y^{b-1} x^{v} e_{2 t+1}^{2 t+1}$ for $0 \leq v \leq a-2$. It is not hard to see that $\operatorname{Ker} \delta_{2 t+1}^{v}$ does not contain any other element $y^{u} x^{v} e_{i}^{2 t+1}$ for which $i$ is odd.

Finally, we count the number of nontrivial linear combinations of two or more basis elements in $\bigoplus_{i=0}^{2 t+1}\left({ }_{\nu} A_{1}\right) e_{i}^{2 t+1}$ belonging to $\operatorname{Ker} \delta_{2 t+1}^{\nu}$. In the homology case, these were

$$
y^{u} x^{v} e_{i}^{2 t+1}+C^{\prime \prime}(a, b, i, u, v) y^{u+1} x^{v-1} e_{i+1}^{2 t+1}
$$

for $0 \leq u \leq b-2,1 \leq v \leq a-1$ and $i=0,2, \ldots, 2 t$, and

$$
y^{b-1} e_{i}^{2 t+1}+C^{\prime \prime \prime}(a, b, i, u, v) x^{a-1} e_{i-1}^{2 t+1}
$$

for $i=2,4, \ldots, 2 t$, where $C^{\prime \prime}$ and $C^{\prime \prime \prime}$ are suitable scalars. The $t$ latter elements also belong to $\operatorname{Ker} \delta_{2 t+1}^{v}$, but among the $(a-1)(b-1)(t+1)$ first elements there are some combinations that are not mapped to zero. Namely, we must discard the $b-1$ elements for which $i=0$ and $v=a-1$, since we showed above that $y^{u} x^{a-1} e_{0}^{2 t+1}$ maps to zero for $0 \leq u \leq b-2$. Similarly, we must discard the $a-1$ combinations for which $i=2 t$ and $u=b-2$, since $y^{b-1} x^{v} e_{2 t+1}^{2 t+1}$ maps to zero for $0 \leq v \leq a-2$. However, when $t=0$, then the situations $i=0$ and $i=2 t$ are the same, and the element

$$
y^{b-2} x^{a-1} e_{0}^{1}+C^{\prime \prime}(a, b, i, u, v) y^{b-1} x^{a-2} e_{1}^{1}
$$

has been discarded twice. Thus the total number of nontrivial linear combinations is $(a-1)(b-1)(t+1)+t-(a-1)-(b-1)$ when $t \geq 1$, and one more when $t=0$.

What happens when char $k$ divides $b$ ? The element $y^{u} x^{a-2} e_{1}^{2 t+1}$ is not mapped to zero for any $u$, and it does not "interfere" with one of the nontrivial linear combinations. Hence the dimension of $\operatorname{Ker} \delta_{2 t+1}^{\nu}$ is also independent of the characteristic of $k$.

In total, we see that the dimension of $\operatorname{Ker} \delta_{2 t+1}^{v}$ differs from that in the homology case, since we need to subtract $(a-1)+(b-1)$ when $t \geq 1$ and $(a-1)+(b-1)-1$ when $t=0$. Thus, the dimension is given by

$$
\operatorname{dim}_{k} \operatorname{Ker} \delta_{2 t+1}^{v}= \begin{cases}a b+2, & \text { when } t=0 \\ a b t+a b+1, & \text { when } t \geq 1\end{cases}
$$

We can now calculate the positive degree cohomology groups. We have

$$
\operatorname{dim}_{k} \operatorname{Ker} \delta_{1}^{v}=a b+2,
$$


and, since $\operatorname{dim}_{k} \operatorname{Ker} \delta_{2}^{v}=2 a b$, we must have $\operatorname{dim}_{k} \operatorname{Im} \delta_{2}^{v}=a b$, giving

$$
\operatorname{dim}_{k} \operatorname{HH}^{1}(A)=\operatorname{dim}_{k} \operatorname{Ker} \delta_{1}^{\nu}-\operatorname{dim}_{k} \operatorname{Im} \delta_{2}^{\nu}=2 .
$$

Furthermore, since $\operatorname{dim}_{k} \operatorname{Ker} \delta_{3}^{\nu}=2 a b+1$, we must have $\operatorname{dim}_{k} \operatorname{Im} \delta_{3}^{\nu}=2 a b-1$, giving

$$
\operatorname{dim}_{k} \mathrm{HH}^{2}(A)=\operatorname{dim}_{k} \operatorname{Ker} \delta_{2}^{\nu}-\operatorname{dim}_{k} \operatorname{Im} \delta_{3}^{v}=1 .
$$

Similarly, direct computations show that the cohomology groups $\mathrm{HH}^{n}(A)$ vanish when $n \geq 3$, thereby completing the proof.

When the commutator element $q$ is a root of unity, it is not hard to see that the dimensions of infinitely many of the kernels in the complex we used to compute (co)homology will increase. Therefore, the Hochschild homology of $A$ is still nonzero in all degrees, while it is no longer true that all the higher Hochschild cohomology groups vanish. We record this fact in the final result, which also gives the multiplicative structure of the Hochschild cohomology ring when $q$ is not a root of unity.

Theorem 3.3. The Hochschild cohomology ring $\mathrm{HH}^{*}(A)$ is finite-dimensional if and only if $q$ is not a root of unity. When this is the case, the algebra is isomorphic to the (five-dimensional graded) fibre product

$$
k[U] /\left(U^{2}\right) \times_{k} k\langle V, W\rangle /\left(V^{2}, V W+W V, W^{2}\right),
$$

where $U$ is in degree zero and $V$ and $W$ are in degree one.

Proof. Suppose $q$ is not a root of unity. Recall first the initial part

$$
P_{2} \stackrel{d_{2}}{\rightarrow} P_{1} \stackrel{d_{1}}{\rightarrow} P_{0} \stackrel{\mu}{\rightarrow} A \rightarrow 0
$$

of the projective bimodule resolution of $A$, where $\mu$ is the multiplication map. The maps $d_{1}$ and $d_{2}$ are defined on generators as follows:

$$
\begin{aligned}
d_{1}: & f_{0}^{1} \mapsto[(1 \otimes y)-(y \otimes 1)] f_{0}^{0}, \\
f_{1}^{1} & \mapsto[(1 \otimes x)-(x \otimes 1)] f_{0}^{0}, \\
d_{2}: \quad f_{0}^{2} & \mapsto\left[\left(1 \otimes y^{b-1}\right)+\left(y \otimes y^{b-2}\right)+\cdots+\left(y^{b-1} \otimes 1\right)\right] f_{0}^{1}, \\
f_{1}^{2} & \mapsto[q(1 \otimes x)-(x \otimes 1)] f_{0}^{1}+[(1 \otimes y)-q(y \otimes 1)] f_{1}^{1}, \\
f_{2}^{2} & \mapsto\left[\left(1 \otimes x^{a-1}\right)+\left(x \otimes x^{a-2}\right)+\cdots+\left(x^{a-1} \otimes 1\right)\right] f_{1}^{1} .
\end{aligned}
$$

Define two bimodule maps

$$
\begin{aligned}
& g: P_{1} \rightarrow A,\left\{\begin{array}{l}
f_{0}^{1} \mapsto y, \\
f_{1}^{1} \mapsto 0,
\end{array}\right. \\
& h: P_{1} \rightarrow A,\left\{\begin{array}{l}
f_{0}^{1} \mapsto 0, \\
f_{1}^{1} \mapsto x
\end{array}\right.
\end{aligned}
$$


One checks directly that

$$
g \circ d_{2}=0=h \circ d_{2},
$$

and that neither of the two maps is liftable through $d_{1}$. Consequently they represent the two basis elements of $\operatorname{HH}^{1}(A)=\operatorname{Ext}_{A^{\mathrm{e}}}^{1}(A, A)$.

We may identify the degree zero part of $\mathrm{HH}^{*}(A)$ with the center of $A$, the twodimensional vector space spanned by the elements 1 and $y^{b-1} x^{a-1}$. The latter element annihilates both $g$ and $h$; hence $\operatorname{HH}^{*}(A)$ is isomorphic to the $k$-fibre product of the algebra generated by $y^{b-1} x^{a-1}$ with the algebra generated by $g$ and $h$. Since the Hochschild cohomology ring of a finite-dimensional algebra is always graded commutative (see [Snashall and Solberg 2004, Corollary 1.2]), both $g$ and $h$ square to zero. Therefore, as $\mathrm{HH}^{2}(A)$ is one-dimensional, we are done if we can show that the product $h g \in \mathrm{HH}^{2}(A)$ is nonzero.

Define a bimodule map $g_{0}: P_{1} \rightarrow P_{0}$ by

$$
g_{0}: f_{0}^{1} \mapsto(y \otimes 1) f_{0}^{0}, f_{1}^{1} \mapsto 0 .
$$

It is not hard to see that there exists an element $w \in A^{\mathrm{e}}$ such that the map $g_{1}: P_{2} \rightarrow$ $P_{1}$, defined by

$$
g_{1}: f_{0}^{2} \mapsto w f_{0}^{1}, f_{1}^{2} \mapsto q(y \otimes 1) f_{1}^{1}, f_{2}^{2} \mapsto 0
$$

gives a commutative diagram

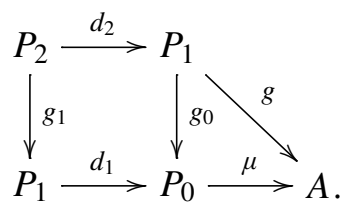

The product $h g \in \mathrm{HH}^{2}(A)$ is then represented by the composite map $h \circ g_{1}$, under which the images of the generators in $P_{2}$ are given by

$$
h \circ g_{1}: f_{0}^{2} \mapsto 0, f_{1}^{2} \mapsto q y x, f_{2}^{2} \mapsto 0 .
$$

This map is not liftable through $d_{2}$, and therefore it represents a nonzero element of $\mathrm{HH}^{2}(A)$. Consequently, the product $h g$ is nonzero.

\section{References}

[Avramov et al. 1997] L. L. Avramov, V. N. Gasharov, and I. V. Peeva, "Complete intersection dimension”, Inst. Hautes Études Sci. Publ. Math. 86 (1997), 67-114 (1998). MR 99c:13033 Zbl 0918.13008

[Benson et al. 2007] D. J. Benson, K. Erdmann, and M. Holloway, "Rank varieties for a class of finite-dimensional local algebras”, J. Pure Appl. Algebra 211:2 (2007), 497-510. MR 2008f:16006 Zbl 05186643 
[Buchweitz et al. 2005] R.-O. Buchweitz, E. L. Green, D. Madsen, and Ø. Solberg, "Finite Hochschild cohomology without finite global dimension", Math. Res. Lett. 12:5-6 (2005), 805-816. MR 2006k:16019 Zbl 05001030

[Cartan and Eilenberg 1956] H. Cartan and S. Eilenberg, Homological algebra, Princeton University Press, Princeton, NJ, 1956. MR 17,1040e Zbl 0075.24305

[Erdmann et al. 2004] K. Erdmann, M. Holloway, R. Taillefer, N. Snashall, and Ø. Solberg, "Support varieties for selfinjective algebras", K-Theory 33:1 (2004), 67-87. MR 2007f:16014 Zbl 1116. 16007

[Green and Snashall 2004] E. L. Green and N. Snashall, "Projective bimodule resolutions of an algebra and vanishing of the second Hochschild cohomology group", Forum Math. 16:1 (2004), 17-36. MR 2005c:16011 Zbl 1074.16003

[Han 2006] Y. Han, "Hochschild (co)homology dimension”, J. London Math. Soc. (2) 73:3 (2006), 657-668. MR 2007c:16018 Zbl 05044873

[Happel 1989] D. Happel, "Hochschild cohomology of finite-dimensional algebras", pp. 108-126 in Séminaire d'Algèbre Paul Dubreil et Marie-Paul Malliavin, 39ème Année (Paris, 1987/1988), edited by M.-P. Malliavin, Lecture Notes in Math. 1404, Springer, Berlin, 1989. MR 91b:16012 Zbl 0688.16033

[Holm 2000] T. Holm, "Hochschild cohomology rings of algebras $k[X] /(f)$ ", Beiträge Algebra Geom. 41:1 (2000), 291-301. MR 2001d:13014 Zbl 0961.13006

[Manin 1987] Y. I. Manin, "Some remarks on Koszul algebras and quantum groups", Ann. Inst. Fourier (Grenoble) 37:4 (1987), 191-205. MR 89e:16022 Zbl 0625.58040

[Snashall and Solberg 2004] N. Snashall and Ø. Solberg, "Support varieties and Hochschild cohomology rings", Proc. London Math. Soc. (3) 88:3 (2004), 705-732. Zbl 1067.16010

Communicated by Dave Benson

Received 2007-10-15 Revised 2008-05-29 Accepted 2008-06-07

bergh@math.ntnu.no

erdmann@maths.ox.ac.uk
Institutt for matematiske fag, NTNU, N-7491 Trondheim, Norway http://www.math.ntnu.no/ bergh

Mathematical Institute, 24-29 St. Giles, Oxford, OX13LB, United Kingdom

http://www.maths.ox.ac.uk/ erdmann 


\title{
Tate resolutions for Segre embeddings
}

\author{
David A. Cox and Evgeny Materov
}

\begin{abstract}
We give an explicit description of the terms and differentials of the Tate resolution of sheaves arising from Segre embeddings of $\mathbb{P}^{a} \times \mathbb{P}^{b}$. We prove that the maps in this Tate resolution are either coming from Sylvester-type maps, or from Bezout-type maps arising from the so-called toric Jacobian.
\end{abstract}

\section{Introduction}

Let $V$ and $W$ be dual vector spaces of dimension $N+1$ over a field $K$ of characteristic 0 . It is known that there is a relation between complexes of free graded modules over the exterior algebra $E=\bigwedge V$ and coherent sheaves on projective space $\mathbb{P}(W)$. More precisely, the Bernstein-Gel'fand-Gel'fand (BGG) correspondence [1978] establishes an equivalence between the derived category of bounded complexes of coherent sheaves on $\mathbb{P}(W)$ and the stable category of complexes of finitely generated graded modules over $E$. The essential part of this correspondence is given via the Tate resolutions, namely for any coherent sheaf $\mathscr{F}$ on $\mathbb{P}(W)$ there exists a bi-infinite exact sequence

$$
T^{\bullet}(\mathscr{F}): \cdots \rightarrow T^{-1}(\mathscr{F}) \rightarrow T^{0}(\mathscr{F}) \rightarrow T^{1}(\mathscr{F}) \rightarrow \cdots
$$

of free graded $E$-modules. The terms of Tate resolution were described explicitly by Eisenbud, Fløystad and Schreyer [2003a] in the form

$$
T^{p}(\mathscr{F})=\bigoplus_{i} \widehat{E}(i-p) \otimes H^{i}(\mathbb{P}(W), \mathscr{F}(p-i)),
$$

where

$$
\widehat{E}=\omega_{E}=\operatorname{Hom}_{K}(E, K)=\bigwedge W
$$

as an $E$-module.

While the terms of Tate resolutions are described explicitly, the maps are much more difficult to describe. The knowledge of the maps give us, for example, an

MSC2000: primary 13D02; secondary 14M25.

Keywords: Tate resolution, Segre embedding, toric Jacobian.

E. Materov was partially supported by the Russian Foundation for Basic Research, grant 05-0100517, by grant 06-01-91063 from the Japanese Society for the Promotion of Science and the Russian Foundation for Basic Research, and by NM project 45.2007 of the Siberian Federal University grant. 
opportunity to compute generalized resultants (see, for example, [Eisenbud et al. 2003b] or [Khetan 2003; 2005]).

Cox [2007] found an explicit construction of the Tate resolution for the $d$-fold Veronese embedding

$$
v_{d}: \mathbb{P}^{n} \rightarrow \mathbb{P}^{\left(\begin{array}{c}
n+d \\
d
\end{array}\right)-1}
$$

of $\mathbb{P}^{n}$ when $\mathscr{F}_{F}=v_{d *} \mathscr{O}_{\mathbb{P}^{n}}(k)$. The construction of differentials in Tate resolution involves the Bezoutian of $n+1$ homogeneous polynomials of degree $d$ in $n+1$ variables. In this paper, we find a similar description of the Tate resolution arising from the Segre embedding

$$
v: \mathbb{P}^{a} \times \mathbb{P}^{b} \rightarrow \mathbb{P}^{a b+a+b}
$$

of the sheaf $v_{*} \mathcal{O}_{\mathbb{p} a \times \mathbb{P}^{b}}(k, l)$. The shape of the Tate resolution depends only on the pair $(k, l)$ and there are three types of possible resolutions:

$$
\begin{array}{ll}
\text { Type } 1: & -a \leq k-l \leq b, \\
\text { Type 2: } & k-l>b, \\
\text { Type 3: } & k-l<-a .
\end{array}
$$

We prove that Type 1 maps involve the toric Jacobian of a sequence bilinear forms $f_{0}, \ldots, f_{a+b}$ in $x_{0}, \ldots, x_{a}, y_{0}, \ldots, y_{b}$ given by

$$
f_{j}=\sum_{i, k} a_{i j k} x_{i} y_{k}, \quad 0 \leq j \leq a+b .
$$

This result resembles the Bezout-type formulas for hyperdeterminants of a threedimensional matrix $A=\left(a_{i j k}\right)$ discussed in [Gel'fand et al. 1994, Chapter 14, Theorem 3.19]. The resolutions of Type 2 and 3 are similar to each other and both arise from the Sylvester forms of $f_{0}, \ldots, f_{a+b}$. Notice that similar formulas appear in the study of Bondal type formulas for hyperdeterminants of $A$ (see [Gel'fand et al. 1994, Chapter 14, Theorem 3.18]).

The situations considered in this paper and [Cox 2007] are special cases when $\mathscr{F}$ is a push-forward of $\mathscr{L}=\mathscr{O}\left(m_{1}, \ldots, m_{r}\right)$ in the projective embedding

$$
v: \mathbb{P}^{l_{1}} \times \cdots \times \mathbb{P}^{l_{r}} \rightarrow \mathbb{P}\left(S^{d_{1}} K^{l_{1}+1} \otimes \cdots \otimes S^{d_{r}} K^{l_{r}+1}\right)
$$

which is a combination of Segre and Veronese embeddings. This case will be studied in a different paper [Cox and Materov $\geq 2008$ ]. We conjecture that the maps in the Tate resolutions are essentially the same as in Weyman-Zelevinsky complexes [1994] or the same as in the resultant spectral sequences from [Gel'fand et al. 1994, Chapter 4, Section 3].

Here is the outline of our paper. In Section 2 we give a definition of the Tate resolution and explain its basic properties. We then enter the main part of the 
paper: in Section 3 we describe the terms of Tate resolution arising from Segre embeddings of sheaves on products of projective spaces, and in Section 4 we find explicit forms for corresponding differentials.

\section{Basic definitions and properties of Tate resolutions}

2.1. Graded exterior algebras. Given $V$ and $W$ as above, the algebras $S=\operatorname{Sym} W$ and $E=\bigwedge V$ are graded by the following convention: $\operatorname{deg}\left(e_{i}\right)=1$ for a basis $e_{0}, e_{1}, \ldots, e_{N}$ of $W$ and $\operatorname{deg}\left(e_{i}^{*}\right)=-1$ for the dual basis $e_{0}^{*}, e_{1}^{*}, \ldots, e_{N}^{*}$ of $V$, so that $E_{-i}=\bigwedge^{i} V$. Define $E(p)$ as the graded $E$-module with $E(p)_{q}=E_{p+q}$. Then any free graded $E$-module is an $E$-module of the form

$$
M=\bigoplus_{i} E(-i) \otimes V_{i}
$$

where $V_{i}$ is a finite-dimensional $K$-vector space with $V_{i}=\{0\}$ for almost all $i$. Note that $V_{i}$ gives the degree $i$ generators of $M$, because

$$
\left(E(-i) \otimes V_{i}\right)_{i}=E(-i)_{i} \otimes V_{i}=E_{0} \otimes V_{i}=V_{i} .
$$

The dual to $E$ algebra $\widehat{E}=\omega_{E}=\operatorname{Hom}_{K}(E, K)$ is a left $E$-module with the graded components

$$
\widehat{E}_{i}=\operatorname{Hom}_{K}\left(E_{-i}, K\right)=\operatorname{Hom}_{K}\left(\bigwedge^{i} V, K\right) .
$$

The perfect pairing

$$
\bigwedge^{i} V \times \bigwedge^{i} W \rightarrow K
$$

implies $\widehat{E}_{i}=\bigwedge^{i} W$ and $\widehat{E}=\bigwedge W$. Moreover, $\widehat{E}$ is Gorenstein, that is, $\widehat{E}$ is isomorphic to $E$ with a shift in grading. Namely, the isomorphism

$$
\bigwedge^{i} V \otimes \bigwedge^{N+1} W \rightarrow \bigwedge^{N+1-i} W
$$

implies

$$
\widehat{E}=E(-N-1) \otimes \bigwedge^{N+1} W,
$$

and therefore $\widehat{E} \cong E(-N-1)$ (noncanonically) via a map $\bigwedge^{N+1} W \cong K$. For later purposes, we note the canonical isomorphism

$$
\operatorname{Hom}_{E}(\widehat{E}(p) \otimes A, \widehat{E}(q) \otimes B)_{0} \simeq \operatorname{Hom}_{K}\left(\bigwedge^{p-q} W \otimes A, B\right),
$$

where the subscript 0 denotes graded homomorphisms of degree zero. 
2.2. Tate resolutions. By [Eisenbud et al. 2003a] or [Fløystad 2000] a coherent sheaf $\mathscr{F}$ on $\mathbb{P}(W)$ determines a Tate resolution $T^{\bullet}(\mathscr{F})$, which is an (unbounded) acyclic complex

$$
T^{\bullet}(\mathscr{F}): \cdots \rightarrow T^{-1}(\mathscr{F}) \rightarrow T^{0}(\mathscr{F}) \rightarrow T^{1}(\mathscr{F}) \rightarrow \cdots
$$

of free graded $E$-modules with the terms

$$
T^{p}(\mathscr{F})=\bigoplus_{i} \widehat{E}(i-p) \otimes H^{i}(\mathbb{P}(W), \mathscr{F}(p-i)) .
$$

For example, in degree $k$ we have

$$
T^{p}(\mathscr{F})_{k}=\bigoplus_{i} \bigwedge^{i-p+k} W \otimes H^{i}(\mathbb{P}(W), \mathscr{F}(p-i))
$$

since $\widehat{E}(i-p)_{k}=\widehat{E}_{i-p+k}=\bigwedge^{i-p+k} W$. The Tate resolution is defined by each differential $d^{p}: T^{p}(\mathscr{F}) \rightarrow T^{p+1}(\mathscr{F})$ since $T^{\geq p}(\mathscr{F})$ is a minimal injective resolution of $\operatorname{ker}\left(d^{p}\right)$ and $T^{<p}(\mathscr{F})$ is a minimal projective resolution of $\operatorname{ker}\left(d^{p}\right)$ [Eisenbud 2005].

When the context is clear, we will write $H^{i}(\mathscr{F}(j))$ instead of $H^{i}(\mathbb{P}(W), \mathscr{F}(j))$.

Lemma 2.1. For fixed $k, T^{p}(\mathscr{F})_{k}=0$ if either $p>k+m$ or $p<k-N-1$, where $m=\operatorname{dim}(\operatorname{supp}(\mathscr{F}))$.

Proof. Since $H^{i}(\mathscr{F}(p-i))=0$ if $i<0$ or $i>m$, we may assume $0 \leq i \leq m$. Then the inequalities $k+m<p, i \leq m$ easily imply

$$
i-p+k \leq m-p+k<-p+p=0,
$$

so that $\bigwedge^{i-p+k} W=0$. Analogously, if $k-N-1>p, i \geq 0$, then

$$
i-p+k \geq-p+k>-p+p+N+1=N+1,
$$

so that we have again $\bigwedge^{i-p+k} W=0$.

Lemma 2.2. If $i<j$, then the map

$$
d_{i, j}^{p}: \widehat{E}(i-p) \otimes H^{i}(\mathscr{F}(p-i)) \rightarrow \widehat{E}(j-p-1) \otimes H^{j}(\mathscr{F}(p+1-j))
$$

from the $i$-th summand of $T^{p}(\mathscr{F})$ to the $j$-th summand of $T^{p+1}(\mathscr{F})$ is zero.

Proof. Let

$$
A=H^{i}(\mathscr{F}(p-i)) \text { and } B=H^{j}(\mathscr{F}(p+1-j)) .
$$

By $(2-1), d_{i, j}^{p}$ lies in

$\operatorname{Hom}_{E}(\widehat{E}(i-p) \otimes A, \widehat{E}(j-p-1) \otimes B)_{0} \simeq \operatorname{Hom}_{K}\left(\bigwedge^{i-j+1} W \otimes A, B\right)$. 
It follows that $d_{i, j}^{p}=0$ when $i+1<j$ and that $d_{i, i+1}^{p}$ is constant. Then minimality implies that $d_{i, i+1}^{p}=0$.

Finding an explicit expression for differentials $d^{p}: T^{p}(\mathscr{F}) \rightarrow T^{p+1}(\mathscr{F})$ seems to be a difficult problem. By Lemma 2.2, the general maps from the $i$-th summand of $T^{p}(\mathscr{F})$ in the Tate resolution $T^{\bullet}(\mathscr{F})$ have the form

$$
\widehat{E}(i-p) \otimes H^{i}(\mathscr{F}(p-i)) \rightarrow \bigoplus_{j \geq 0} \widehat{E}(i-j-p-1) \otimes H^{i-j}(\mathscr{F}(p+1-i+j)) .
$$

The "horizontal" cmponent of this map is explicitly known:

$$
\begin{aligned}
\widehat{E}(i-p) \otimes H^{i}(\mathscr{F}(p-i)) & \rightarrow \widehat{E}(i-p-1) \otimes H^{i}(\mathscr{F}(p+1-i)), \\
f \otimes m & \mapsto \sum_{i} f e_{i}^{*} \otimes e_{i} m .
\end{aligned}
$$

By (2-1), this corresponds to the multiplication map

$$
W \otimes H^{i}(\mathscr{F}(p-i)) \rightarrow H^{i}(\mathscr{F}(p+1-i)) .
$$

One of the main results of this paper is an explicit description the entire differential $d^{p}: T^{p}(\mathscr{F}) \rightarrow T^{p+1}(\mathscr{F})$ in some special situations.

\section{Tate resolutions for Segre embeddings of $\mathbb{P}^{a} \times \mathbb{P}^{b}$}

Let $X=\mathbb{P}^{a} \times \mathbb{P}^{b}$, with coordinate ring $S=K[\mathbf{x}, \mathbf{y}]$ for variables $\mathbf{x}=\left(x_{0}, \ldots, x_{a}\right)$, $\mathbf{y}=\left(y_{0}, \ldots, y_{b}\right)$. The ring $S$ has a natural bigrading where the $\mathbf{x}$ variables have degree $(1,0)$ and the $\mathbf{y}$ variables have degree $(0,1)$. The graded piece of $S$ in degree $s, t$ will be denoted $S_{s, t}$. Set

$$
W=H^{0}\left(X, \mathscr{O}_{X}(1,1)\right)=S_{1,1}
$$

and let

$$
v: X=\mathbb{P}^{a} \times \mathbb{P}^{b} \rightarrow \mathbb{P}(W) \simeq \mathbb{P}^{a b+a+b}
$$

be the Segre embedding. The sheaf

$$
\mathscr{F}=v_{*} \mathcal{O}_{X}(k, l)
$$

has Tate resolution $T^{\bullet}(\mathscr{F})$ with

$$
\begin{aligned}
T^{p}(\mathscr{F}) & =\bigoplus_{i} \widehat{E}(i-p) \otimes H^{i}(\mathscr{F}(p-i)) \\
& =\bigoplus_{i} \widehat{E}(i-p) \otimes H^{i}\left(X, \bigcirc_{X}(k+p-i, l+p-i)\right)
\end{aligned}
$$


In general, we say that the summand $\widehat{E}(i-p) \otimes H^{i}(\mathscr{F}(p-i))$ of $T^{p}(\mathscr{F})$ has cohomological level $i$. Since

$$
H^{i}\left(X, O_{X}(k+p-i, l+p-i)\right)=0
$$

for $i \notin\{0, a, b, a+b\}$, we see that $T^{p}(\mathscr{F})$ has at most four nonzero cohomological levels.

In Section 2.2, we observed that the "horizontal" components of the differentials $d^{p}: T^{p}(\mathscr{F}) \rightarrow T^{p+1}(\mathscr{F})$ are explicitly known. The main result of this paper is a description of the "diagonal" components of these maps.

3.1. Regularity. We recall that a coherent sheaf $\mathscr{F}$ is called $m$-regular if

$$
H^{i}(\mathscr{F}(m-i))=0, \quad \text { for all } i>0 .
$$

If $\mathscr{F}$ is $m$-regular, then it is known that it is also $(m+1)$-regular. The regularity of $\mathscr{F}$, denoted reg(F) , is the unique integer $m$ such that $\mathscr{F}$ is $m$-regular, but not $(m-1)$-regular. It follows from the definition of regularity if $m=\operatorname{reg}(\mathscr{F})$, then

$$
T^{p}(\mathscr{F})=\widehat{E}(-p) \otimes H^{0}(\mathscr{F}(p)), \quad \text { for } p \geq m,
$$

and the Tate resolution has the form

$$
\cdots \rightarrow T^{m-2}(\mathscr{F}) \rightarrow T^{m-1}(\mathscr{F}) \rightarrow \widehat{E}(-m) \otimes H^{0}(\mathscr{F}(m)) \rightarrow \cdots .
$$

We now compute the regularity of the sheaf $\mathscr{F}$ defined in (3-1).

Lemma 3.1. $\operatorname{reg}(\mathscr{F})=\max \{-\min \{k, l\}, \min \{b-k, a-l\}\}$.

Proof. Let $m_{0}$ denote the right-hand side of the above equation and let $m \geq m_{0}$. Then Serre duality implies

$$
\begin{aligned}
H^{a+b}(\mathscr{F}(m-(a+b))) & =H^{a+b}\left(X, \mathcal{O}_{X}(k+m-(a+b), l+m-(a+b))\right) \\
& \simeq H^{0}\left(X, O_{X}(b-k-(m+1), a-l-(m+1))\right)^{*} .
\end{aligned}
$$

Since $m \geq m_{0}$ implies $m \geq b-k$ or $m \geq a-l$, we see that $H^{a+b}(\mathscr{F}(m-(a+b)))=0$.

Next we use the Künneth formula to write

$$
\begin{aligned}
H^{a}(\mathscr{F}(m-a))= & H^{a}\left(X, \mathcal{O}_{X}(k+m-a, l+m-a)\right) \\
= & H^{0}\left(\mathbb{P}^{a}, \mathcal{O}(k+m-a)\right) \otimes H^{a}\left(\mathbb{P}^{b}, \mathcal{O}(l+m-a)\right) \oplus \\
& H^{a}\left(\mathbb{P}^{a}, \mathcal{O}(k+m-a)\right) \otimes H^{0}\left(\mathbb{P}^{b}, \mathcal{O}(l+m-a)\right) .
\end{aligned}
$$

Since $m \geq m_{0}$ implies $m \geq-k$ and $m \geq-l$, we see that $k+m-a \geq-a$, which implies $H^{a}\left(\mathbb{P}^{a}, \mathcal{O}(k+m-a)\right)=0$. Furthermore, $H^{a}\left(\mathbb{P}^{b}, \mathcal{O}(l+m-a)\right)=0$ when $a \neq b$, and when $a=b$, we have $l+m-a=l+m-b \geq-b$, which again implies $H^{a}\left(\mathbb{P}^{b}, \mathcal{O}(l+m-a)\right)=0$. Hence $H^{a}(\mathscr{F}(m-a))=0$, and $H^{b}(\mathscr{F}(m-b))=0$ is proved similarly. 
It follows that $m_{0} \geq \operatorname{reg}(\mathscr{F})$. To prove equality, we will let $m=m_{0}-1$ and show that $H^{i}(\mathscr{F}(m-i)) \neq 0$ for some $i>0$. We consider two cases.

Case 1: $m_{0}=\min \{b-k, a-l\} \geq-\min \{k, l\}$. This implies the inequalities $b-k-(m+1) \geq 0$ and $a-l-(m+1) \geq 0$. Hence

$$
H^{a+b}(\mathscr{F}(m-(a+b))) \simeq H^{0}\left(X, \mathcal{O}_{X}(b-k-(m+1), a-l-(m+1))\right)^{*} \neq 0 .
$$

Case 2: $m_{0}=-\min \{k, l\}>\min \{b-k, a-l\}$. If $m_{0}=-k$, then $k+m-a=$ $-a-1$, so that $H^{a}\left(\mathbb{P}^{a}, \mathcal{O}(k+m-a)\right) \neq 0$. We also have $m_{0}>\min \{b-k, a-l\}$, so that $m_{0}>b-k$ or $m_{0}>a-l$. The former is impossible since $m_{0}=-k$, and then the latter implies $l+m-a \geq 0$, so that $H^{0}\left(\mathbb{P}^{b}, \mathcal{O}(l+m-a)\right) \neq 0$. By Künneth,

$$
0 \neq H^{a}\left(\mathbb{P}^{a}, \mathcal{O}(k+m-a)\right) \otimes H^{0}\left(\mathbb{P}^{b}, \mathcal{O}(l+m-a)\right) \subseteq H^{a}(\mathscr{F}(m-a)) .
$$

The proof when $m_{0}=-l$ is similar.

To see what this says about the Tate resolution of $\mathscr{F}$, we define

$$
\begin{aligned}
& p^{+}=\max \{-\min \{k, l\}, \min \{b-k, a-l\}\}, \\
& p^{-}=\min \{-\min \{k, l\}, \min \{b-k, a-l\}\}-1 .
\end{aligned}
$$

Then we have the following result.

\section{Lemma 3.2.}

$$
T^{p}(\mathscr{F})= \begin{cases}\widehat{E}(-p) \otimes S_{k+p, l+p} & p \geq p^{+} \\ \widehat{E}(a+b-p) \otimes S_{b-k-1-p, a-l-1-p}^{*} & p \leq p^{-} .\end{cases}
$$

Proof. The assertion for $p \geq p^{+}$follows immediately from Lemma 3.1 and the discussion preceding the lemma. For $p \leq p^{-}$, note that

$$
\begin{aligned}
H^{a+b}(\mathscr{F}(p-(a+b))) & \simeq H^{0}\left(X, \mathcal{O}_{X}(b-k-(p+1), a-l-(p+1))\right)^{*} \\
& =S_{b-k-1-p, a-k-1-p}^{*}
\end{aligned}
$$

and that

$$
\begin{aligned}
H^{a+b-i}(\mathscr{F}(p-(a+b-i))) & \simeq H^{i}\left(X, \mathcal{O}_{X}(b-k-1-p-i, a-l-1-p-i)\right)^{*} \\
& =H^{i}(\mathscr{G}(-p-i)),
\end{aligned}
$$

where $\mathscr{G}=v_{*} \mathrm{O}_{X}(b-k-1, a-l-1)$. Applying Lemma 3.1 to $\mathscr{G}$, we see that $H^{i}(\varphi(-p-i))=0$ whenever $i>0$ and

$$
-p \geq \max \{-\min \{b-k-1, a-l-1\}, \min \{b-(b-k-1), a-(a-l-1)\}\},
$$

which is equivalent to $p \leq p^{-}$.

Lemma 3.2 tells us that for $p^{-}$and below, the Tate resolution lives at cohomological level $a+b$, and for $p^{+}$and above, it lives at cohomological level 0 . 
3.2. The shape of the resolution. For $k, l \in \mathbb{Z}$, the Tate resolution of

$$
\mathscr{F}=v_{*} O_{X}(k, l)
$$

on $X=\mathbb{P}^{a} \times \mathbb{P}^{b}$ has one of the following three types:

Type 1: $\quad-a \leq k-l \leq b$,

Type 2: $k-l>b$,

Type 3: $k-l<-a$.

We will prove three lemmas, one for each type.

Lemma 3.3 (Type 1). Assume that F has Type 1 . Then $p^{-}=-\min \{k, l\}-1$ and $p^{+}=\min \{b-k, a-l\}$. Furthermore, if $p^{-}<p<p^{+}$, then

$$
T^{p}(\mathscr{F})=\begin{gathered}
\widehat{E}(a+b-p) \otimes S_{b-k-1-p, a-l-1-p}^{*} \\
\bigoplus \\
\widehat{E}(-p) \otimes S_{k+p, l+p} .
\end{gathered}
$$

Proof. Since $a$ and $b$ are positive, the inequality $-a \leq k-l \leq b$ implies that $-\min \{k, l\} \leq \min \{b-k, a-l\}$. Using (3-2), we get the desired formulas for $p^{-}$ and $p^{+}$.

Now assume that $p^{-}<p<p^{+}$. Recall that $H^{a}(\mathscr{F}(p-a))$ is isomorphic to

$$
\begin{aligned}
H^{0}\left(\mathbb{P}^{a}, \mathcal{O}(k+p-a)\right) \otimes H^{a}\left(\mathbb{P}^{b}, \mathcal{O}(l+p-a)\right) \\
\qquad H^{a}\left(\mathbb{P}^{a}, \mathcal{O}(k+p-a)\right) \otimes H^{0}\left(\mathbb{P}^{b}, \mathcal{O}(l+p-a)\right) .
\end{aligned}
$$

If the second summand is nonzero, then $k+p-a<-a$ and $l+p-a \geq 0$, which implies $k-l<-a$, a contradiction. If the first summand is nonzero, then $a=b$, $k+p-a \geq 0$ and $l+p-a<-a$. These imply $k-l>a=b$, again a contradiction. Hence $H^{a}(\mathscr{F}(p-a))=0$. A similar argument shows that $H^{b}(\mathscr{F}(p-b))=0$.

Thus, when $\mathscr{F}$ has Type 1 , the differential $d^{p}: T^{p}(\mathscr{F}) \rightarrow T^{p+1}(\mathscr{F})$ looks like

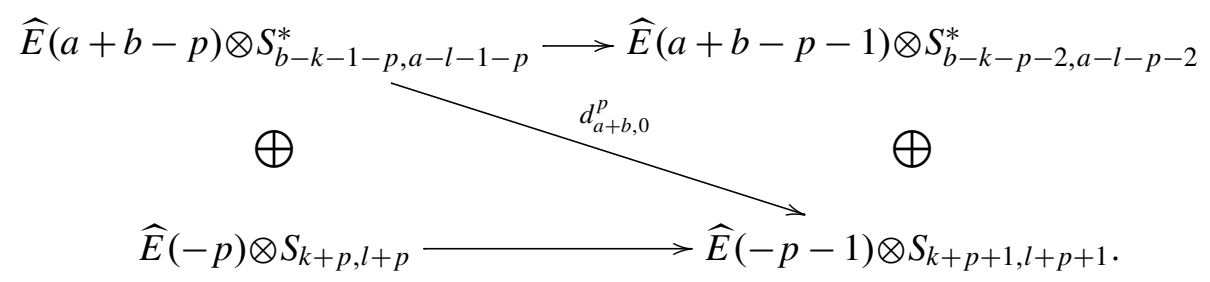

Hence a Type 1 Tate resolution has cohomological levels $a+b$ (the top row) and 0 (the bottom row). Section 4.1 will discuss $d_{a+b, 0}^{p}$. 
Lemma 3.4 (Type 2). Assume that F्F has Type 2. Then $p^{-}=b-k-1$ and $p^{+}=-l$. Furthermore, if $p^{-}<p<p^{+}$, then

$$
T^{p}(\mathscr{F})=\widehat{E}(b-p) \otimes S_{k+p-b, 0} \otimes S_{0,-l-p-1}^{*} .
$$

Proof. Since $a$ and $b$ are positive, the inequality $k-l>b$ implies $\min \{k, l\}=l$, $\min \{b-k, a-l\}=b-k$. Using $k-l>b$ again, (3-2) gives the desired formulas for $p^{-}$and $p^{+}$.

Now assume that $p^{-}<p<p^{+}$. Then

$$
H^{a+b}(\mathscr{F}(p-(a+b))) \simeq H^{0}\left(X, \mathcal{O}_{X}(b-k-1-p, a-l-1-p)\right)^{*}=0
$$

since $p>p^{-}=b-k-1$. Furthermore, $p<p^{+}=-l$ implies $l+p-b<0$, so that

$$
H^{b}\left(\mathbb{P}^{a}, \mathcal{O}(k+p-b)\right) \otimes H^{0}\left(\mathbb{P}^{b}, \mathcal{O}(l+p-b)\right)=0 .
$$

Hence, by Künneth and Serre duality on $\mathbb{P}^{b}$,

$$
\begin{aligned}
H^{b}(\mathscr{F}(p-b)) & \simeq H^{b}\left(X, \mathcal{O}_{X}(k+p-b, l+p-b)\right) \\
& \simeq H^{0}\left(\mathbb{P}^{a}, \mathcal{O}(k+p-b)\right) \otimes H^{b}\left(\mathbb{P}^{b}, \mathcal{O}(l+p-b)\right) \\
& \simeq S_{k+p-b, 0} \otimes S_{0,-l-p-1}^{*} .
\end{aligned}
$$

Finally, if $a \neq b$, we also have

$$
H^{a}\left(\mathbb{P}^{b}, \mathcal{O}(l+p-a)\right)=0,
$$

and $H^{0}\left(\mathbb{P}^{b}, \mathcal{O}(l+p-a)\right)=0$ also holds since $l+p-a<0$. Hence $H^{a}(\mathscr{F}(p-a))=0$ when $a \neq b$. A similar argument shows that $H^{0}(\mathscr{F}(p))=0$.

Lemma 3.4 tells us that for Type 2 Tate resolutions, the only nonzero diagonal maps appear in $T^{p^{-}}(\mathscr{F}) \rightarrow T^{p^{-}+1}(\mathscr{F})$ :

$$
\widehat{E}(a+1+k) \otimes \underbrace{d^{-}}_{0, a+k-l-b \quad S_{a+b, b}^{*}}(k) \otimes S_{0,0} \otimes S_{0, k-l-b-1}^{*}
$$

(at cohomological levels $a+b$ and $b$ ) and in $T^{p^{+}-1}(\mathscr{F}) \rightarrow T^{p^{+}}(\mathscr{F})$ :

$$
\widehat{E}(b+1+l) \otimes S_{k-l-b-1,0} \otimes S_{0,0}^{*} \underbrace{d_{b, 0}^{p^{+}-1}}
$$

(at cohomological levels $b$ and 0 ). The diagonal maps $d_{a+b, b}^{p^{-}}$and $d_{b, 0}^{p^{+}-1}$ will be discussed in Section 4.2. 
Lemma 3.5 (Type 3). Assume that F has Type 3. Then $p^{-}=a-l-1$ and $p^{+}=-k$. Furthermore, if $p^{-}<p<p^{+}$, then

$$
T^{p}(\mathscr{F})=\widehat{E}(a-p) \otimes S_{-k-p-1,0}^{*} \otimes S_{0, l+p-a} .
$$

Proof. The proof is similar to the proof of Lemma 3.4 and hence is omitted.

Lemma 3.5 tells us that for Type 3 Tate resolutions, the only nonzero diagonal maps appear in $T^{p^{-}}(\mathscr{F}) \rightarrow T^{p^{-}+1}(\mathscr{F})$ :

$$
\widehat{E}(b+1+l) \otimes S_{b-k+l-a, 0}^{*} \underbrace{d_{a+b, a}^{p^{-}}}_{\widehat{E}(l)} \otimes S_{l-k-a-1,0}^{*} \otimes S_{0,0}
$$

(at cohomological levels $a+b$ and $a$ ) and in $T^{p^{+}-1}(\mathscr{F}) \rightarrow T^{p^{+}}(\mathscr{F})$ :

$$
\widehat{E}(a+1+k) \otimes S_{0,0}^{*} \otimes \underbrace{S_{0, l-k-a-1} d_{a, 0}^{p^{+}-1}}(k) \otimes S_{0, l-k}
$$

(at cohomological levels $a$ and 0 ). The diagonal maps $d_{a+b, a}^{p^{-}}$and $d_{a, 0}^{p^{+}-1}$ will be discussed in Section 4.2.

Remark 3.6. We finish this section by noting that some of the Tate resolutions considered here can be found in [Fløystad 2004]. Specifically, let $W_{1}$ and $W_{2}$ be finite-dimensional $K$-vector spaces, and consider the Tate resolution associated to $\mathscr{F}=v_{*} \mathscr{L}$, where

$$
\mathscr{L}=\mathcal{O}_{\mathbb{P}}\left(W_{1}\right) \times \mathbb{P}\left(W_{2}\right)(-2, a) \otimes \bigwedge^{a+1} W_{1},
$$

$\operatorname{dim} W_{1}=a+1$, and

$$
v: \mathbb{P}\left(W_{1}\right) \times \mathbb{P}\left(W_{2}\right) \rightarrow \mathbb{P}\left(W_{1} \otimes W_{2}\right)
$$

is the Segre embedding. The results of our paper apply to this Tate resolution.

Now consider a surjective map $W_{1}^{*} \otimes W_{2}^{*} \rightarrow W^{*}$. This gives a projection

$$
\pi: \mathbb{P}\left(W_{1} \otimes W_{2}\right) \rightarrow \mathbb{P}(W)
$$

whose center is disjoint from the image of the Segre map. By [Fløystad 2004, Section 1.2], the Tate resolution for $\mathscr{F}$ gives a Tate resolution for $\mathscr{G}=\pi_{*} \mathscr{F}$. Fløystad shows that this projected Tate resolution has the form

$\cdots \rightarrow T^{-1}(\varphi) \rightarrow T^{0}(\varphi)=\widehat{E}(a) \otimes W_{1}^{*} \rightarrow T^{1}(\varphi)=\widehat{E}(a-1) \otimes W_{2}^{*} \rightarrow T^{2}(\varphi) \rightarrow \cdots$ with the map $d^{0}: T^{0}(\mathscr{G}) \rightarrow T^{1}(\mathscr{G})$ coming from the surjection $W_{1}^{*} \otimes W_{2}^{*} \rightarrow W^{*}$ [Fløystad 2004, Theorem 2.1]. 


\section{The maps in the Tate resolution for Segre embeddings of $\mathbb{P}^{a} \times \mathbb{P}^{b}$}

4.1. Type 1 diagonal maps. We will use the toric Jacobian from [Cox 1996, §4]. The fan for $\mathbb{P}^{a} \times \mathbb{P}^{b}$ has $a+b+21$-dimensional cone generators

$$
e_{0}, \ldots, e_{a}, e_{0}^{\prime}, \ldots, e_{b}^{\prime}
$$

corresponding to $x_{0}, \ldots, x_{a}, y_{0}, \ldots, y_{b}$. The generators $e_{1}, \ldots, e_{a}, e_{0}^{\prime}, \ldots, e_{b-1}^{\prime}$ are linearly independent. Given $f_{0}, \ldots, f_{a+b} \in S_{1,1}$, the toric Jacobian is

$$
J\left(f_{0}, \ldots, f_{a+b}\right)=\frac{1}{x_{0} y_{b}} \operatorname{det}\left(\begin{array}{ccc}
f_{0} & \cdots & f_{a+b} \\
\partial f_{0} / \partial x_{1} & \cdots & \partial f_{a+b} / \partial x_{1} \\
\vdots & & \vdots \\
\partial f_{0} / \partial x_{a} & \cdots & \partial f_{a+b} / \partial x_{a} \\
\partial f_{0} / \partial y_{0} & \cdots & \partial f_{a+b} / \partial y_{0} \\
\vdots & & \vdots \\
\partial f_{0} / \partial y_{b-1} & \cdots & \partial f_{a+b} / \partial y_{b-1}
\end{array}\right) .
$$

Since $f_{i} \in S_{1,1}=W$, we see that $J\left(f_{0}, \ldots, f_{a+b}\right) \in S_{b, a}$, where $(b, a)$ is the "critical degree," often denoted $\rho$ in the literature on toric residues.

This toric Jacobian is closely related to the $(a+1) \times(a+b+1) \times(b+1)$ hyperdeterminant discussed in [Gel'fand et al. 1994, 14.3.D]. The connection becomes especially clear when we use the graph interpretation from [Gel'fand et al. 1994, pp. 473-474]. The idea is as follows.

Fix distinct monomials $f_{0}, \ldots, f_{a+b} \in S_{1,1}$. These give a bipartite graph $G$ with $a+b+2$ vertices $x_{0}, \ldots, x_{a}, y_{0}, \ldots, y_{b}$ and $a+b+1$ edges given by the monomials, where $f_{\ell}=x_{i} y_{j}$ is regarded as the edge connecting $x_{i}$ to $y_{j}$. The incidence matrix of $G$ is the $(a+b+2) \times(a+b+1)$ matrix whose rows correspond to vertices and columns correspond to edges, and where an entry is 1 if the vertex lies on the edge and is 0 otherwise.

Let $M$ denote the square matrix obtained from the incidence matrix by removing the bottom row. Then we have the following result.

Lemma 4.1. Let $f_{0}, \ldots, f_{a+b} \in S_{1,1}$ be distinct monomials and let $M$ be the matrix described above.

(1) The toric Jacobian of $f_{0}, \ldots, f_{a+b}$ is given by

$$
J\left(f_{0}, \ldots, f_{a+b}\right)=\operatorname{det} M \frac{\prod_{\ell} f_{\ell}}{\prod_{i} x_{i} \prod_{j} y_{j}} .
$$

(2) $\operatorname{det} M \in\{0, \pm 1\}$, and $\operatorname{det} M= \pm 1$ if and only if $G$ is a tree. 
Proof. Each $f_{\ell}$ is homogeneous of degree 1 in $x_{0}, \ldots, x_{a}$, so $f_{\ell}=\sum_{i} x_{i} \frac{\partial f_{\ell}}{\partial x_{i}}$. Hence the toric Jacobian $J\left(f_{0}, \ldots, f_{a+b}\right)$ can be written as

$\frac{1}{x_{0} y_{b}} \operatorname{det}\left(\begin{array}{ccc}x_{0} \partial f_{0} / \partial x_{0} & \cdots & x_{0} \partial f_{a+b} / \partial x_{0} \\ \partial f_{0} / \partial x_{1} & \cdots & \partial f_{a+b} / \partial x_{1} \\ \vdots & & \vdots \\ \partial f_{0} / \partial x_{a} & \cdots & \partial f_{a+b} / \partial x_{a} \\ \partial f_{0} / \partial y_{0} & \cdots & \partial f_{a+b} / \partial y_{0} \\ \vdots & & \vdots \\ \partial f_{0} / \partial y_{b-1} & \cdots & \partial f_{a+b} / \partial y_{b-1}\end{array}\right)$

$$
=\frac{1}{\prod_{i} x_{i} \prod_{j} y_{j}} \operatorname{det}\left(\begin{array}{ccc}
x_{0} \partial f_{0} / \partial x_{0} & \cdots & x_{0} \partial f_{a+b} / \partial x_{0} \\
x_{1} \partial f_{0} / \partial x_{1} & \cdots & x_{1} \partial f_{a+b} / \partial x_{1} \\
\vdots & & \vdots \\
x_{a} \partial f_{0} / \partial x_{a} & \cdots & x_{a} \partial f_{a+b} / \partial x_{a} \\
y_{0} \partial f_{0} / \partial y_{0} & \cdots & y_{0} \partial f_{a+b} / \partial y_{0} \\
\vdots & & \vdots \\
y_{b-1} \partial f_{0} / \partial y_{b-1} & \cdots & y_{b-1} \partial f_{a+b} / \partial y_{b-1}
\end{array}\right) .
$$

For a fixed $\ell$, we have $f_{\ell}=x_{i} y_{j}$, which implies

$$
f_{\ell}=x_{i} \frac{\partial f_{\ell}}{\partial x_{i}}=y_{j} \frac{\partial f_{\ell}}{\partial y_{j}},
$$

and all other partials vanish. Hence the $\ell$-th column is a multiple of $f_{\ell}$, and once we factor out $f_{\ell}$, we are left with the $\ell$-th column of the truncated incidence matrix $M$. Thus

$$
J\left(f_{0}, \ldots, f_{a+b}\right)=\frac{f_{0} \cdots f_{a+b}}{\prod_{i} x_{i} \prod_{j} y_{j}} \operatorname{det}(M) .
$$

The second part of the lemma is a standard consequence of the Matrix Tree Theorem [Bondy and Murty 1981, Chapter 12] which counts the number of spanning trees of a graph.

Now that we have the toric Jacobian, the next step is to introduce duplicate sets of variables

$$
\mathbf{X}=\left(X_{0}, \ldots, X_{a}\right), \mathbf{Y}=\left(Y_{0}, \ldots, Y_{b}\right), \mathbf{x}=\left(x_{0}, \ldots, x_{a}\right), \mathbf{y}=\left(y_{0}, \ldots, y_{b}\right) .
$$

These give the polynomial ring

$$
S \otimes S=k[\mathbf{X}, \mathbf{Y}, \mathbf{x}, \mathbf{y}]
$$


and the ring homomorphism

$$
S=k[\mathbf{x}, \mathbf{y}] \rightarrow S \otimes S
$$

defined by $x_{i} \mapsto X_{i}+x_{i}, y_{i} \mapsto Y_{i}+y_{i}$. The image of $F \in S$ in $S \otimes S$ is denoted $\widetilde{F}$, so that

$$
\widetilde{F}(\mathbf{X}, \mathbf{Y}, \mathbf{x}, \mathbf{y})=F(\mathbf{X}+\mathbf{x}, \mathbf{Y}+\mathbf{y}) \in S \otimes S .
$$

From a canonical point of view, the map $F \mapsto \widetilde{F}$ is comultiplication in the natural Hopf algebra structure on $S$.

The toric Jacobian $J$ gives a linear map

$$
J: \bigwedge^{a+b+1} W \rightarrow S_{b, a} \subset S
$$

and hence a map

$$
\widetilde{J}: \bigwedge^{a+b+1} W \rightarrow S \otimes S .
$$

Looking at homogeneous pieces, we have a decomposition

$$
\widetilde{J}=\bigoplus_{\alpha, \beta} J_{\alpha, \beta}
$$

where

$$
J_{\alpha, \beta}: \bigwedge^{a+b+1} W \rightarrow S_{b-\alpha, a-\beta} \otimes S_{\alpha, \beta}
$$

lies in

$$
\operatorname{Hom}_{K}\left(\bigwedge^{a+b+1} W, S_{b-\alpha, a-\beta} \otimes S_{\alpha, \beta}\right) \simeq \operatorname{Hom}_{K}\left(\bigwedge^{a+b+1} W \otimes S_{b-\alpha, a-\beta}^{*}, S_{\alpha, \beta}\right) .
$$

Using (2-1), $J_{\alpha, \beta}$ gives an element of

$$
\operatorname{Hom}_{E}\left(\widehat{E}(a+b-p) \otimes S_{b-\alpha, a-\beta}^{*}, \widehat{E}(-p-1) \otimes S_{\alpha, \beta}\right),
$$

which by abuse of notation we write as

$$
J_{\alpha, \beta}: \widehat{E}(a+b-p) \otimes S_{b-\alpha, a-\beta}^{*} \rightarrow \widehat{E}(-p-1) \otimes S_{\alpha, \beta} .
$$

In Section 4.3 we will show that the map $d_{a+b, 0}^{p}$ from a Type 1 Tate resolution (see the discussion of following Lemma 3.3) can be chosen to be $J_{k+p+1, l+p+1}$.

4.2. Type 2 and 3 diagonal maps. The diagonal maps appearing the Type 2 and 3 Tate resolutions discussed in Section 3.2 are easy to describe. We begin with the map

$$
\delta: \bigwedge^{a+1} W \rightarrow S_{0, a+1}
$$

defined as follows: given $f_{0}, \ldots, f_{a} \in W$, we get the Sylvester form

$$
\delta\left(f_{0}, \ldots, f_{a}\right)=\operatorname{det}\left(\ell_{i j}\right),
$$

where $f_{i}=\sum_{j=0}^{a} \ell_{i j} x_{j}$ for $\ell_{i j} \in S_{0,1}$. 
Now fix $\alpha \geq 0$. The multiplication map $S_{0, a+1} \otimes S_{0, \alpha} \rightarrow S_{0, a+1+\alpha}$ induces

$$
S_{0, a+1} \rightarrow S_{0, \alpha}^{*} \otimes S_{0, a+1+\alpha}
$$

and gives the composition

$$
\bigwedge^{a+1} W \stackrel{\delta}{\rightarrow} S_{0, a+1} \rightarrow S_{0, \alpha}^{*} \otimes S_{0, a+1+\alpha} .
$$

This gives maps

$$
\begin{aligned}
& \delta_{\alpha}: \bigwedge^{a+1} W \otimes S_{0, \alpha} \rightarrow S_{0, a+1+\alpha}, \\
& \delta_{\alpha}^{*}: \bigwedge^{a+1} W \otimes S_{0, a+1+\alpha}^{*} \rightarrow S_{0, \alpha}^{*}
\end{aligned}
$$

and hence (by abuse of notation) maps

$$
\begin{aligned}
& \delta_{\alpha}: \widehat{E}(a+1+k) \otimes S_{0, \alpha} \rightarrow \widehat{E}(k) \otimes S_{0, a+1+\alpha}, \\
& \delta_{\alpha}^{*}: \widehat{E}(a+1+k) \otimes S_{0, a+1+\alpha}^{*} \rightarrow \widehat{E}(k) \otimes S_{0, \alpha}^{*} .
\end{aligned}
$$

In Section 4.3 we will show that the diagonal map $d_{a+b, b}^{p^{-}}$from a Type 2 Tate resolution (see the discussion following Lemma 3.4) and the map $d_{a, 0}^{p^{+}-1}$ from a Type 3 Tate resolution (see the discussion following Lemma 3.5) can be chosen to be $\delta_{k-l-b-1}^{*}$ and $\delta_{l-k-a-1}$ respectively.

We next consider the map

$$
\delta^{\prime}: \bigwedge^{b+1} W \rightarrow S_{b+1,0}
$$

defined as follows: given $f_{0}, \ldots, f_{b} \in W$,

$$
\delta^{\prime}\left(f_{0}, \ldots, f_{a}\right)=\operatorname{det}\left(\ell_{i j}^{\prime}\right),
$$

where $f_{i}=\sum_{j=0}^{b} \ell_{i j}^{\prime} y_{j}$ for $\ell_{i j}^{\prime} \in S_{1,0}$.

As above, $\beta \geq 0$ gives the multiplication map $S_{b+1,0} \otimes S_{\beta, 0} \rightarrow S_{b+1+\beta, 0}$ and the composition

$$
\bigwedge^{b+1} W \stackrel{\delta^{\prime}}{\longrightarrow} S_{b+1,0} \rightarrow S_{\beta, 0}^{*} \otimes S_{b+1+\beta, 0} .
$$

This gives maps

$$
\begin{aligned}
& \delta_{\beta}^{\prime}: \widehat{E}(b+1+l) \otimes S_{\beta, 0} \rightarrow \widehat{E}(l) \otimes S_{b+1+\beta, 0}, \\
& \delta_{\beta}^{\prime *}: \widehat{E}(b+1+l) \otimes S_{b+1+\beta, 0}^{*} \rightarrow \widehat{E}(l) \otimes S_{\beta, 0}^{*} .
\end{aligned}
$$

In Section 4.3 we will show that the map $d_{b, 0}^{p^{+}-1}$ from a Type 2 Tate resolution (see the discussion following Lemma 3.4) and the map $d_{a+b, a}^{p^{-}}$from a Type 3 Tate resolution (see the discussion following Lemma 3.5) can be chosen to be $\delta_{k-l-b-1}^{\prime}$ and $\delta_{l-k-a-1}^{*}$ respectively. 
4.3. The main theorem. Here is the main result of this section.

Theorem 4.2. For the Tate resolution $T^{\bullet}(\mathscr{F})$ of the sheaf $\mathscr{F}=v_{*} \mathrm{O}_{X}(k, l)$, the diagonal maps in $T^{p}(\mathscr{F}) \rightarrow T^{p+1}(\mathscr{F})$ can be chosen as follows:

(1) (Type $1,-a \leq k-l \leq b)$ : $d_{a+b, 0}^{p}=(-1)^{p} J_{k+p+1, l+p+1}$.

(2) (Type 2, $k-l>b$ ): $d_{a+b, b}^{p^{-}}=\delta_{k-l-b-1}^{*}$ and $d_{b, 0}^{p^{+}-1}=\delta_{k-l-b-1}^{\prime}$.

(3) (Type 3, $k-l<-a$ ): $d_{a+b, a}^{p^{-}}=\delta_{l-k-a-1}^{*}$ and $d_{a, 0}^{p^{+}-1}=\delta_{l-k-a-1}$.

This uses the maps $J_{\alpha, \beta}, \delta_{\alpha}, \delta_{\alpha}^{*}, \delta_{\beta}^{\prime}, \delta_{\beta}^{*}$ defined in (4-2), (4-3) and (4-4).

Proof. We begin with Type 2 . Let $\beta=k-l-b-1$ and assume $l=0$ for simplicity, so that $p^{+}=0$. We will show that

$$
T^{-2}(\mathscr{F}) \rightarrow T^{-1}(\mathscr{F}) \rightarrow T^{0}(\mathscr{F}) \rightarrow T^{1}(\mathscr{F})
$$

can be constructed as follows using $\delta_{\beta}^{\prime}$ :

$$
\begin{aligned}
\widehat{E}(b+2) \otimes S_{\beta-1,0} \otimes S_{0,1}^{*} \stackrel{d^{-2}}{\rightarrow} \widehat{E}(b+1) & S_{\beta, 0} \otimes S_{0,0}^{*} \\
& \widehat{E}(0) \otimes S_{\beta+b+1,0}^{\delta_{\beta}^{\prime}} \stackrel{d^{0}}{\rightarrow} \widehat{E}(-1) \otimes S_{\beta+b+2,1} .
\end{aligned}
$$

The differentials $d^{-2}$ and $d^{0}$ are the known horizontal maps. To show that this sequence is exact, the first step is to prove that

$$
d^{0} \circ \delta_{\beta}^{\prime}=\delta_{\beta}^{\prime} \circ d^{-2}=0 .
$$

Consider the following identity that holds for all $f_{0}, \ldots, f_{b+1} \in W$ :

$$
\sum_{i=0}^{b+1}(-1)^{i} f_{i} \delta^{\prime}\left(f_{0} \wedge \cdots \widehat{f}_{i} \cdots \wedge f_{b+1}\right)=0 .
$$

If we write $f_{i}=\sum_{j=0}^{b} \ell_{i j}^{\prime} y_{j}$, then (4-5) follows from the obvious identity

$$
\operatorname{det}\left(\begin{array}{ccc}
f_{0} & \ldots & f_{b+1} \\
\ell_{0,0}^{\prime} & \ldots & \ell_{b+1,0}^{\prime} \\
\vdots & & \vdots \\
\ell_{0, b}^{\prime} & \cdots & \ell_{b+1, b}^{\prime}
\end{array}\right)=0
$$

by expanding by minors along the first row and using the definition of $\delta^{\prime}$.

By (2-1), the composition

$$
\widehat{E}(b+1) \otimes S_{\beta, 0} \otimes S_{0,0}^{*} \stackrel{\delta_{\beta}^{\prime}}{\rightarrow} \widehat{E}(0) \otimes S_{\beta+b+1,0} \stackrel{d^{0}}{\rightarrow} \widehat{E}(-1) \otimes S_{\beta+b+2,1}
$$


corresponds to a map

$$
\bigwedge^{b+2} W \otimes S_{\beta, 0} \otimes S_{0,0}^{*} \rightarrow S_{\beta+b+2,1}
$$

We ignore $S_{0,0}^{*} \simeq k$. Using the definition of $\delta_{\beta}^{\prime}$, this map is given by

$$
f_{0} \wedge \cdots \wedge f_{b+1} \otimes h \mapsto \sum_{i=0}^{b+1}(-1)^{i} f_{i} h \delta^{\prime}\left(f_{0} \wedge \cdots \widehat{f}_{i} \cdots \wedge f_{b+1}\right)
$$

This reduces to zero (factor out $h \in S_{\beta, 0}$ and use (4-5)), so $d^{0} \circ \delta_{\beta}^{\prime}=0$.

If $\beta>0$, we need to consider $\delta_{\beta}^{\prime} \circ d^{-2}$. Arguing as above, we determine this map by

$$
\bigwedge^{b+2} W \otimes S_{\beta-1,0} \otimes S_{0,1}^{*} \rightarrow S_{\beta+b+1,0},
$$

which in turn is determined by the map

$$
\bigwedge^{b+2} W \otimes S_{\beta-1,0} \rightarrow S_{\beta+b+1,0} \otimes S_{0,1}=S_{\beta+b+1,1}
$$

given by

$$
f_{0} \wedge \cdots \wedge f_{b+1} \otimes h \mapsto \sum_{i=0}^{b+1}(-1)^{i} f_{i} h \delta^{\prime}\left(f_{0} \wedge \cdots \widehat{f_{i}} \cdots \wedge f_{b+1}\right)
$$

for $h \in S_{\beta-1,0}$. As above, this reduces to zero, so that $\delta_{\beta}^{\prime} \circ d^{-2}=0$.

When $\beta=0$, we have to show that the composition

$$
\widehat{E}(a+b+2) \otimes S_{0, a+1}^{*} \delta_{0}^{*}(a+1) \otimes S_{0,0} \otimes \underbrace{S_{0,0}^{*}}_{\widehat{E}(0) \otimes S_{b+1,0}}
$$

is zero. By (2-1), the composed map corresponds to a map

$$
\bigwedge^{a+b+2} W \otimes S_{0, a+1}^{*} \rightarrow S_{b+1,0},
$$

which in turn is determined by the a map

$$
\bigwedge^{a+b+2} W \rightarrow S_{b+1,0} \otimes S_{0, a+1}=S_{b+1, a+1} .
$$

Given $f_{0}, \ldots, f_{a+b+1} \in W$, this map is given by

$$
f_{0} \wedge \cdots \wedge f_{a+b+1} \mapsto \sum_{|S|=a+1} \varepsilon(S) \delta\left(\mathbf{f}_{S}\right) \delta^{\prime}\left(\mathbf{f}_{S^{c}}\right)
$$


where the sum is over all subsets $S \subset\{0, \ldots, a+b+1\}$ of cardinality $a+1$ and $S^{c}=\{0, \ldots, a+b+1\} \backslash S$. Furthermore,

$$
\begin{gathered}
\delta\left(\mathbf{f}_{S}\right)=\delta\left(\bigwedge_{i \in S} f_{i}\right), \\
\delta^{\prime}\left(\mathbf{f}_{S^{c}}\right)=\delta^{\prime}\left(\bigwedge_{i \in S^{c}} f_{i}\right),
\end{gathered}
$$

and $\varepsilon(S)= \pm 1$ is the sign that appears in the Laplace expansion described below.

To show that the sum in (4-6) is zero, write

$$
f_{i}=\sum_{j=0}^{a} \ell_{i j} x_{j}=\sum_{j=0}^{b} \ell_{i j}^{\prime} y_{j}
$$

and consider the matrix

$$
\mathcal{M}=\left(\begin{array}{ccc}
\ell_{0,0} & \cdots & \ell_{a+b+1,0} \\
\vdots & & \vdots \\
\ell_{0, a} & \cdots & \ell_{a+b+1, a} \\
\ell_{0,0}^{\prime} & \cdots & \ell_{a+b+1,0}^{\prime} \\
\vdots & & \vdots \\
\ell_{0, b}^{\prime} & \cdots & \ell_{a+b+1, b}^{\prime}
\end{array}\right) .
$$

If we multiply first $a+1$ rows by suitable $\mathbf{x}$ variables and multiply the last $b+1$ rows by $\mathbf{y}$ variables, we get the same result, namely the row $\left(f_{0}, \ldots, f_{a+b+1}\right)$. If follows that $\operatorname{det} M=0$. If we take the Laplace expansion that involves $(a+1) \times(a+1)$ minors of the first $a+1$ rows multiplied by $(b+1) \times(b+1)$ complementary minors of the last $b+1$ rows, we get the sum in (4-6). Hence this sum is zero, which proves that $\delta_{0}^{\prime} \circ \delta_{0}^{*}=0$.

To complete the proof that $\delta_{\beta}^{\prime}$ gives the diagonal map in $T^{-1}(\mathscr{F}) \rightarrow T^{0}(\mathscr{F})$, we follow the strategy used in [Cox 2007, Theorem 1.3]. Let $N^{\prime}=(a+1)(b+1)=$ $\operatorname{dim}(W)$. Since $\widehat{E} \simeq E\left(-N^{\prime}\right)$ and $T^{-1}(\mathscr{F}) \rightarrow T^{0}(\mathscr{F}) \rightarrow T^{1}(\mathscr{F})$ is

$$
\widehat{E}(b+1) \otimes S_{\beta, 0} \otimes S_{0,0}^{*} \rightarrow \widehat{E}(0) \otimes S_{\beta+b+1,0} \stackrel{d^{0}}{\rightarrow} \widehat{E}(-1) \otimes S_{\beta+b+2,1},
$$

the kernel of $d^{0}$ has $\operatorname{dim}\left(S_{\beta, 0} \otimes S_{0,0}^{*}\right)$ minimal generators of degree $N^{\prime}-b-1$. Since we have proved that $\delta_{\beta}^{\prime}$ maps into this kernel, it suffices to prove that this map is injective in degree $N^{\prime}-b-1$, namely that

$$
\delta_{\beta}^{\prime}: \bigwedge^{N^{\prime}} W \otimes S_{\beta, 0} \rightarrow \bigwedge^{N^{\prime}-b-1} W \otimes S_{\beta+b+1,0}
$$

is injective (as above, we ignore $S_{0,0}^{*}$ ). A basis of $\bigwedge^{N^{\prime}} W$ is given by

$$
x_{0} y_{0} \wedge \cdots \wedge x_{0} y_{b} \wedge \omega
$$


where $\omega$ is the wedge product of the remaining $N^{\prime}-b-1$ monomials of $W$ in some order. Since

$$
\delta^{\prime}\left(x_{0} y_{0} \wedge \cdots \wedge x_{0} y_{b}\right)=x_{0}^{b+1}
$$

we see that for $h \in S_{\beta, 0}$,

$$
\delta_{\beta}^{\prime}\left(x_{0} y_{0} \wedge \cdots \wedge x_{0} y_{b} \wedge \omega \otimes h\right)=\omega \otimes x_{0}^{b+1} h+\cdots \in \bigwedge^{N^{\prime}-b-1} W \otimes S_{\beta+b+1,0},
$$

where the omitted terms involve basis elements of $\bigwedge^{N^{\prime}-b-1} W$ different from $\omega$. The desired injectivity is now obvious.

This completes the proof for $d_{b, 0}^{p^{+}-1}$ in a Type 2 Tate resolution when $l=0$ and $k=\beta+b+1$. The proof for arbitrary $l$ is similar, and the same proof easily adapts to $d_{a, 0}^{p^{+}-1}$ in a Type 3 Tate resolution. As for $d^{p^{-}}$, we note that applying $\operatorname{Hom}_{E}(-, K) \otimes_{K} \widehat{E}$ to $T^{p}(\mathscr{F})$ gives $T^{a+b-p}(\mathscr{G})$, where $\mathscr{G}=v_{*} \mathcal{O}_{X}(-a-1-k,-b-$ $1-l)$. This duality interchanges Type 2 and Type 3 resolutions. Then our results for

$$
d_{b, 0}^{p^{+}-1} \text { and } \quad d_{a, 0}^{p^{+}-1}
$$

and dualize to give the desired results for

$$
d_{a+b, a}^{p^{-}} \text {and } \quad d_{a+b, b}^{p^{-}} .
$$

It remains to consider Type 1 Tate resolutions. This case will be more complicated since there are two sets of variables to keep track of: the original variables $\mathbf{x}, \mathbf{y}$ and the duplicates $\mathbf{X}, \mathbf{Y}$ introduced in Section 4.1.

Let $\alpha=k+p+1$ and $\beta=k+p+1$. We will show that the crucial part of $T^{p}(\mathscr{F}) \rightarrow T^{p+1}(\mathscr{F}) \rightarrow T^{p+2}(\mathscr{F})$ can be chosen to be

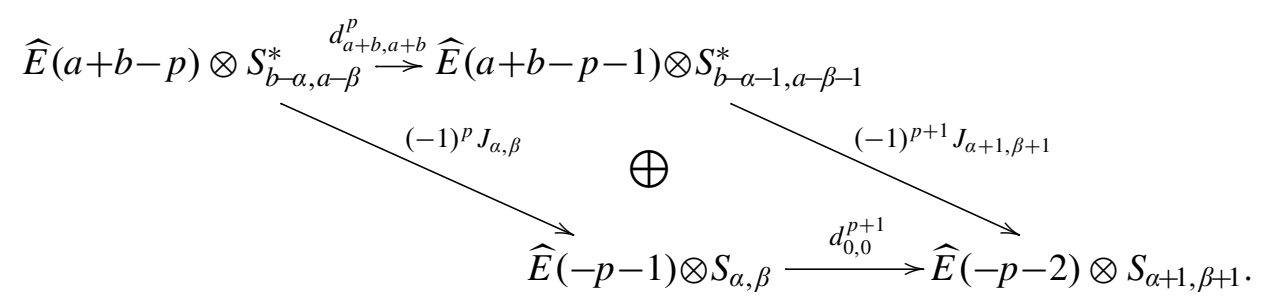

This first step is to show that this is a complex, that is, the composition

$$
T^{p}(\mathscr{F}) \rightarrow T^{p+1}(\mathscr{F}) \rightarrow T^{p+2}(\mathscr{F})
$$

is zero. Since the horizontal maps behave properly, it suffices to show that

$$
d_{0,0}^{p+1} \circ J_{\alpha, \beta}=J_{\alpha+1, \beta+1} \circ d_{a+b, a+b}^{p} .
$$


Using (2-1), this is equivalent to showing that the diagram

$$
\begin{aligned}
& \bigwedge^{a+b+2} W \otimes S_{b-\alpha, a-\beta}^{*} \stackrel{d_{a+b, a+b}^{p}}{\longrightarrow} \bigwedge^{a+b+1} W \otimes S_{b-\alpha-1, a-\beta-1}^{*} \\
& W \stackrel{\downarrow}{J_{\alpha, \beta}} S_{\alpha, \beta} \longrightarrow d_{0,0}^{p+1} \quad S_{\alpha+1, \beta+1}
\end{aligned}
$$

commutes. A key point is that on the top, $d_{a+b, a+b}^{p}$ uses $\mathbf{X}, \mathbf{Y}$, while on the bottom, $d_{0,0}^{p+1}$ uses $\mathbf{x}, \mathbf{y}$. We can recast the commutativity of this diagram as saying that

$$
d_{0,0}^{p+1} \circ J_{\alpha, \beta}=J_{\alpha+1, \beta+1} \circ d_{a+b, a+b}^{p}
$$

as maps

$$
\bigwedge^{a+b+2} W \rightarrow \underbrace{S_{b-\alpha, a-\beta}}_{\mathbf{X}, \mathbf{Y}} \otimes \underbrace{S_{\alpha+1, \beta+1}}_{\mathbf{x}, \mathbf{y}} .
$$

Given $a+b+2$ elements of $W$, we write them as $f_{0}, \ldots, f_{a+b+1}$ when using $\mathbf{x}, \mathbf{y}$ and as $F_{0}, \ldots, F_{a+b+1}$ when using $\mathbf{X}, \mathbf{Y}$. Then (4-7) is equivalent to the identity

$$
\begin{aligned}
\sum_{i=0}^{a+b+1}(-1)^{i} f_{i} J_{\alpha, \beta}\left(f_{0} \wedge \cdots \widehat{f}_{i}\right. & \left.\cdots \wedge f_{a+b+1}\right) \\
& =\sum_{i=0}^{a+b+1}(-1)^{i} F_{i} J_{\alpha+1, \beta+1}\left(f_{0} \wedge \cdots \widehat{f}_{i} \cdots \wedge f_{a+b+1}\right)
\end{aligned}
$$

in $S_{b-\alpha, a-\beta} \otimes S_{\alpha+1, \beta+1}$. Summing this over all $\alpha$ and $\beta$ gives the second identity

$$
\begin{gathered}
\sum_{i=0}^{a+b+1}(-1)^{i} f_{i} \widetilde{J}\left(f_{0} \wedge \cdots \widehat{f}_{i} \cdots \wedge f_{a+b+1}\right) \\
=\sum_{i=0}^{a+b+1}(-1)^{i} F_{i} \widetilde{J}\left(f_{0} \wedge \cdots \widehat{f}_{i} \cdots \wedge f_{a+b+1}\right),
\end{gathered}
$$

and the first identity follows from the second by taking the appropriate graded piece. However,

- The change of variables $(\mathbf{x}, \mathbf{y}) \leftrightarrow(\mathbf{X}, \mathbf{Y})$ interchanges $f_{i}$ and $F_{i}$;

- $\widetilde{J}\left(f_{0} \wedge \cdots \widehat{f}_{i} \cdots \wedge f_{a+b+1}\right)$ is invariant under $(\mathbf{x}, \mathbf{y}) \leftrightarrow(\mathbf{X}, \mathbf{Y})$.

It follows that the second identity is equivalent to the following

Assertion 4.3.

$$
\sum_{i=0}^{a+b+1}(-1)^{i} f_{i} \widetilde{J}\left(f_{0} \wedge \cdots \widehat{f}_{i} \cdots \wedge f_{a+b+1}\right)
$$


is invariant under $(\mathbf{x}, \mathbf{y}) \leftrightarrow(\mathbf{X}, \mathbf{Y})$.

In particular, (4-7) is an immediate consequence of Assertion 4.3.

We will prove Assertion 4.3 by representing (4-8) as a determinant. We begin with the formula

$$
J\left(f_{0} \wedge \cdots \wedge f_{a+b}\right)=\frac{1}{y_{b}} \operatorname{det}\left(\begin{array}{ccc}
\partial f_{0} / \partial x_{0} & \cdots & \partial f_{a+b} / \partial x_{0} \\
\vdots & & \vdots \\
\partial f_{0} / \partial x_{a} & \cdots & \partial f_{a+b} / \partial x_{a} \\
\partial f_{0} / \partial y_{0} & \cdots & \partial f_{a+b} / \partial y_{0} \\
\vdots & & \vdots \\
\partial f_{0} / \partial y_{b-1} & \cdots & \partial f_{a+b} / \partial y_{b-1}
\end{array}\right),
$$

which follows from the proof of Lemma 4.1. This implies

$$
\widetilde{J}\left(f_{0} \wedge \cdots \wedge f_{a+b}\right)=\frac{1}{Y_{b}+y_{b}} \operatorname{det}\left(\begin{array}{ccc}
\widetilde{\partial f_{0} / \partial x_{0}} & \cdots & \partial \widetilde{f_{a+b} / \partial x_{0}} \\
\vdots & & \vdots \\
\widetilde{\partial f_{0} / \partial x_{a}} & \cdots & \partial \widetilde{f_{a+b} / \partial x_{a}} \\
\widetilde{\partial f_{0} / \partial y_{0}} & \cdots & \partial \widetilde{f_{a+b} / \partial y_{0}} \\
\vdots & & \vdots \\
\widetilde{\partial f_{0} / \partial y_{b}-1} & \cdots & \partial \widetilde{f_{a+b} / \partial y_{b-1}}
\end{array}\right) .
$$

It follows easily that

$$
\sum_{i=0}^{a+b+1}(-1)^{i} f_{i} \widetilde{J}\left(f_{0} \wedge \cdots \widehat{f}_{i} \cdots \wedge f_{a+b+1}\right)=\frac{1}{Y_{b}+y_{b}} \operatorname{det} \mathbf{M}
$$

where $\mathbf{M}$ is the $(a+b+2) \times(a+b+2)$ matrix

$$
\mathbf{M}=\left(\begin{array}{ccc}
\frac{f_{0}}{\partial f_{0} / \partial x_{0}} & \cdots & \partial f_{a+b+1} / \partial x_{0} \\
\vdots & & \vdots \\
\frac{f_{a+b+1}}{\partial f_{0} / \partial x_{a}} & \cdots & \partial f_{a+b+1} / \partial x_{a} \\
\frac{\partial f_{0} / \partial y_{0}}{\sigma_{0}} & \cdots & \partial f_{a+b+1} / \partial y_{0} \\
\frac{\vdots}{\partial f_{0} / \partial y_{b-1}} & \cdots & \partial f_{a+b+1} / \partial y_{b-1}
\end{array}\right) .
$$

To prove Assertion 4.3, it suffices to show that the determinant of the matrix $\mathbf{M}$ is unchanged when we replace its top row with $\left(F_{0}, \ldots, F_{a+b+1}\right)$. For this purpose, 
consider the $(a+b+3) \times(a+b+3)$ matrix

$$
\overline{\mathbf{M}}=\left(\begin{array}{cccc} 
& & & 0 \\
& \mathbf{M} & & \vdots \\
\widetilde{\partial f_{0}} & \ldots & \widetilde{\partial f_{a+b+1}} & 1
\end{array}\right)
$$

and observe that $\operatorname{det} \mathbf{M}=\operatorname{det} \overline{\mathbf{M}}$. Write $\overline{\mathbf{M}}$ as

$$
\overline{\mathbf{M}}=\left(\begin{array}{cccc}
f_{0} & \cdots & f_{a+b+1} & 0 \\
& & & 0 \\
& \widetilde{Q} & & \vdots \\
& & & 1
\end{array}\right) .
$$

Since $f_{\ell} \in W=S_{1,1}$, we have the easily proved identity

$$
F_{\ell}-f_{\ell}=-\sum_{i=0}^{a} x_{i} \frac{\widetilde{\partial f_{\ell}}}{\partial x_{i}}+\sum_{j=0}^{b} Y_{j} \frac{\widetilde{\partial f_{\ell}}}{\partial y_{j}} .
$$

Multiplying the last $a+b+2$ rows of $\overline{\mathbf{M}}$ by $-x_{i}$ or $Y_{j}$ as appropriate and adding to the first row gives the matrix

$$
\overline{\mathbf{M}}^{\prime}=\left(\begin{array}{cccc}
F_{0} & \cdots & F_{a+b+1} & Y_{b} \\
& & & 0 \\
& \widetilde{Q} & & \vdots \\
& & & 1
\end{array}\right) .
$$

Note that $\operatorname{det} \overline{\mathbf{M}}^{\prime}=\operatorname{det} \overline{\mathbf{M}}$. This is almost what we need, except for the $Y_{b}$ in the first row of $\overline{\mathbf{M}}^{\prime}$.

We claim that $\operatorname{det} \widetilde{Q}=0$. Assuming this for the moment, it follows that we can replace $Y_{b}$ with 0 in $\overline{\mathbf{M}}^{\prime}$ without changing its determinant. This easily implies $\operatorname{det} \mathbf{M}$ is unchanged when we replace its top row with $\left(F_{0}, \ldots, F_{a+b+1}\right)$ and will complete the proof of (4-7).

It remains to study det $\widetilde{Q}$. The matrix $\widetilde{Q}$ is obtained from

$$
Q=\left(\begin{array}{ccc}
\partial f_{0} / \partial x_{0} & \cdots & \partial f_{a+b} / \partial x_{0} \\
\vdots & & \vdots \\
\partial f_{0} / \partial x_{a} & \cdots & \partial f_{a+b} / \partial x_{a} \\
\partial f_{0} / \partial y_{0} & \cdots & \partial f_{a+b} / \partial y_{0} \\
\vdots & & \vdots \\
\partial f_{0} / \partial y_{b} & \cdots & \partial f_{a+b} / \partial y_{b}
\end{array}\right)
$$


by the $F \mapsto \widetilde{F}$ operation described in Section 4.1. But $\operatorname{det} Q=0$ since $f_{\ell}=$ $\sum_{i=0}^{a} x_{i} \partial f_{\ell} / \partial x_{i}=\sum_{j=0}^{b} y_{j} \partial f_{\ell} / \partial y_{j}$, and then

$$
\operatorname{det} \widetilde{Q}=\widetilde{\operatorname{det} Q}=0 \text {. }
$$

Hence we have proved that the maps

$$
T^{p}(\mathscr{F}) \rightarrow T^{p+1}(\mathscr{F})
$$

defined using $(-1)^{p} J_{\alpha, \beta}$ give a complex. To show that the complex is exact, we again use the strategy of [Cox 2007, Theorem 1.3]. Lemma 3.3 tells us that $p^{+}=$ $\min \{b-k, a-l\}$. For simplicity, we assume $b-k \leq a-l$, so that $p^{+}=b-k$. Let $\beta=b-k+l$ and $p=p^{+}-1$. Type 1 and $b-k \leq a-l$ imply $0 \leq \beta \leq a$. Then $T^{p}(\mathscr{F}) \rightarrow T^{p+1}(\mathscr{F})$ becomes

$$
\begin{gathered}
\widehat{E}(a+k+1) \otimes S_{0, a-\beta}^{*} \\
\widehat{E}(k-b+1) \otimes S_{b-1, \beta-1} \stackrel{d_{0,0}^{p}}{\longrightarrow} \widehat{E}(k-b) \otimes S_{b, \beta} .
\end{gathered}
$$

Let $N^{\prime}=(a+1)(b+1)=\operatorname{dim}(W)$. Then the shape of the Tate resolution tells us that there are $\operatorname{dim}\left(S_{b-1, \beta-1}\right)$ minimal generators of degree $N^{\prime}-(k-b+1)$ and $\operatorname{dim}\left(S_{0, a-\beta}^{*}\right)$ minimal generators of degree $N^{\prime}-(a+k+1)$. The former are taken care of by the known map $d_{0,0}^{p}$, and for the latter, we see that in degree $N^{\prime}-(a+k+1)$, the above diagram becomes

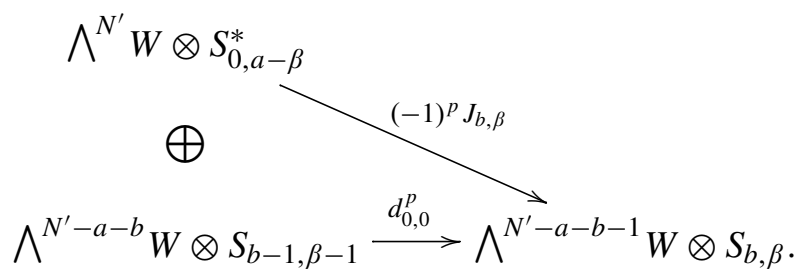

As in [Cox 2007, Lemma 2.2], we need to show that $(-1)^{p} J_{b, \beta}$ is injective and that its image has trivial intersection with the image of $d_{0,0}^{p}$.

For the former, let $\theta \in \bigwedge^{N^{\prime}} W$ be the wedge product of the monomials in $W$ in some order, and let $\varphi \in S_{0, a-\beta}^{*}$ satisfy $J_{b, \beta}(\theta \otimes \varphi)=0$. Suppose that $\mathbf{Y}^{u}$ is a monomial in the $\mathbf{Y}$ variables of degree $|u|=a-\beta$. We prove $\varphi\left(\mathbf{Y}^{u}\right)=0$ as follows.

Pick $\mathbf{Y}^{v}$ such that $\mathbf{Y}^{u} \mid \mathbf{Y}^{v}$ and $|v|=a$, and write

$$
\mathbf{Y}^{v}=Y_{j_{1}} \cdots Y_{j_{a}} .
$$


Then consider the following collection $f_{0}, \ldots, f_{a+b}$ of monomials in $W=S_{1,1}$ :

$$
x_{0} y_{j}, j=0, \ldots, b \text { and } x_{i} y_{j_{i}}, i=1, \ldots, a .
$$

The graph of these monomials (in the sense of Section 4.1) is easily seen to be a tree. Then Lemma 4.1 implies that

$$
J\left(f_{0} \wedge \cdots \wedge f_{a+b}\right)= \pm \frac{\prod_{j=0}^{b} x_{0} y_{j} \prod_{i=1}^{a} x_{i} y_{j_{i}}}{\prod_{i=0}^{a} x_{i} \prod_{j=0}^{b} y_{j}}= \pm x_{0}^{b} \prod_{i=1}^{a} y_{j_{i}}= \pm x_{0}^{b} \mathbf{y}^{v}
$$

Thus $\widetilde{J}\left(f_{0} \wedge \cdots \wedge f_{a+b}\right)= \pm\left(X_{0}+x_{0}\right)^{b}(\mathbf{Y}+\mathbf{y})^{v}$. Taking those terms of degree $(b, \beta)$ in $(\mathbf{x}, \mathbf{y})$, we obtain

$$
J_{b, \beta}\left(f_{0} \wedge \cdots \wedge f_{a+b}\right)= \pm \sum_{w}\left(\begin{array}{l}
v \\
w
\end{array}\right) x_{0}^{b} \mathbf{Y}^{v-w} \mathbf{y}^{w},
$$

where $\left(\begin{array}{l}v \\ w\end{array}\right)=\prod_{j=0}^{b}\left(\begin{array}{c}v_{j} \\ w_{j}\end{array}\right)$ and $\sum_{w}$ denotes the sum over all exponent vectors $w$ satisfying $|w|=\beta$ and $0 \leq w_{j} \leq v_{j}$ for all $j$. Writing $\theta=f_{0} \wedge \cdots \wedge f_{a+b} \wedge \omega$, we obtain

$$
\begin{aligned}
0 & =J_{b, \beta}\left(f_{0} \wedge \cdots \wedge f_{a+b} \wedge \omega \otimes \varphi\right) \\
& =\omega \otimes \varphi\left(J_{b, \beta}\left(f_{0} \wedge \cdots \wedge f_{a+b}\right)\right)+\cdots \\
& =\omega \otimes\left( \pm \sum_{w}\left(\begin{array}{c}
v \\
w
\end{array}\right) \varphi\left(\mathbf{Y}^{v-w}\right) x_{0}^{b} \mathbf{y}^{w}\right)+\cdots,
\end{aligned}
$$

where the omitted terms involve basis elements of $\bigwedge^{N^{\prime}-a-b-1} W$ different from $\omega$. Since we are in characteristic 0 , it follows that $\varphi\left(\mathbf{Y}^{v-w}\right)=0$ for all $w$ under consideration. Our choice of $v$ guarantees that our original monomial $\mathbf{Y}^{u}$ is one of these $\mathbf{Y}^{v-w}$ 's. Hence $\varphi\left(\mathbf{Y}^{u}\right)=0$, which implies $\varphi=0$ since $\mathbf{Y}^{u}$ was an arbitrary monomial of degree $a-\beta$. This completes the proof $(-1)^{p} J_{b, \beta}$ is injective.

It remains to show that the image of this map has trivial intersection with the image of $d_{0,0}^{p}$. Following a suggestion of Jenia Tevelev, we use representation theory to finish the proof.

Recall that there is a natural isomorphism

$$
W=S_{1,1} \cong W_{1} \otimes W_{2},
$$

where $W_{1}=S_{1,0}=\mathbb{C}^{a+1}$ and $W_{2}=S_{0,1}=\mathbb{C}^{b+1}$. First, we show that an action of the group $G=\operatorname{SL}\left(W_{1}\right) \times \operatorname{SL}\left(W_{2}\right)$ on the diagram (4-10) is $G$-invariant on the maps $d_{0,0}^{p}$ and $(-1)^{p} J_{b, \beta}$. Indeed, since the map $d_{0,0}^{p}$ is induced by the multiplication map

$$
W \otimes S_{b-1, \beta-1} \rightarrow S_{b, \beta}
$$


we conclude that $d_{0,0}^{p}$ is $G$-invariant. Now observe that the toric Jacobian can be written as a linear combination of monomials

$$
J\left(f_{0}, \ldots, f_{a+b}\right)=\sum_{\mu, \nu} c_{\mu, \nu} x^{\mu} y^{\nu},
$$

where $c_{\mu, \nu}$ are the entries of the square matrix whose determinant is a hyperdeterminant [Gel'fand et al. 1994, p. 473]. By [Gel'fand et al. 1992, Proposition 1.4], the hyperdeterminant is $G$-invariant, so the toric Jacobian (4-1) (and respectively the map $\left.(-1)^{p} J_{b, \beta}\right)$ is $G$-invariant.

It follows from Schur's Lemma that the images of $d_{0,0}^{p}$ and $J_{b, \beta}$ have trivial intersection if the representation of $G$ corresponding to

$$
\bigwedge^{N^{\prime}-a-b} W \otimes S_{b-1, \beta-1}=\bigwedge^{a b+1}\left(W_{1} \otimes W_{2}\right) \otimes \operatorname{Sym}^{b-1}\left(W_{1}\right) \otimes \operatorname{Sym}^{\beta-1}\left(W_{2}\right)
$$

does not contain the representation corresponding to

$$
\bigwedge^{N^{\prime}} W \otimes S_{0, a-\beta}^{*}=\bigwedge^{a b+a+b+1}\left(W_{1} \otimes W_{2}\right) \otimes \operatorname{Sym}^{a-\beta}\left(W_{2}^{*}\right) .
$$

To prove this, we use some basic facts from the representation theory of the special linear group (see, for example, [Fulton and Harris 1991, §6.1 and §15.3]). Given a partition $\lambda=\left(\lambda_{1}, \ldots, \lambda_{s}\right)$ with $\lambda_{1} \geq \cdots \geq \lambda_{s} \geq 0$, we get a Young diagram $D_{\lambda}$, which consists of $s$ rows of boxes, all starting at the same column, of lengths $\lambda_{1} \geq \cdots \geq \lambda_{s}$.

For a vector space $V$ over $K, \mathbb{S}_{\lambda}(V)$ denotes the irreducible $\operatorname{SL}(V)$-representation corresponding to the partition $\lambda$. We use notation

$$
\lambda=\left(d_{1}^{a_{1}}, \ldots, d_{\ell}^{a_{\ell}}\right)
$$

to denote the partition having $a_{i}$ copies of the integer $d_{i}$ for $1 \leq i \leq \ell$. The corresponding Young diagram $D_{\lambda}$ has $a_{i}$ rows of boxes of length $d_{i}$. Thus $\lambda=(d)$ gives the symmetric product $\mathbb{S}_{\lambda}(V)=\operatorname{Sym}^{d}(V)$ and $\lambda=\left(1^{d}\right)$ gives the exterior product $\mathbb{S}_{\lambda}(V)=\bigwedge^{d} V$.

Recall that $\mathbb{S}_{\lambda}(V)=0$ when the Young diagram of $\lambda$ has more than $\operatorname{dim} V$ nonzero rows, and that two Young diagrams give the same $\operatorname{SL}(V)$-representation if and only if one can be obtained from the other by adding or deleting columns of height $\operatorname{dim} V$ at the beginning of the Young diagram.

By the Cauchy formula [Fulton and Harris 1991, §6.1], we have the following decomposition for the exterior powers of $W=W_{1} \otimes W_{2}$ :

$$
\bigwedge^{a b+1} W=\bigwedge^{a b+1}\left(W_{1} \otimes W_{2}\right)=\bigoplus_{|\lambda|=a b+1} \mathbb{S}_{\lambda}\left(W_{1}\right) \otimes \mathbb{S}_{\lambda^{\prime}}\left(W_{2}\right),
$$

where the direct sum runs over all partitions $\lambda$ of $a b+1$ with at most $\operatorname{dim} W_{1}=a+1$ rows, at most $\operatorname{dim} W_{2}=b+1$ columns, and $\lambda^{\prime}$ is the conjugate partition to $\lambda$. Note 
that the representation corresponding to the highest power of determinant $\bigwedge^{N^{\prime}} W$ is one-dimensional, that is, a trivial representation.

When we combine this with (4-11), we see that it is enough to show that it cannot happen simultaneously that $\mathbb{S}_{\lambda}\left(W_{1}\right) \otimes \operatorname{Sym}^{b-1}\left(W_{1}\right)$ contains the trivial representation and $\mathbb{S}_{\lambda^{\prime}}\left(W_{2}\right) \otimes \operatorname{Sym}^{\beta-1}\left(W_{2}\right)$ contains $\operatorname{Sym}^{a-\beta}\left(W_{2}^{*}\right)$. Since $\operatorname{dim} W_{1}=a+1$ and $\operatorname{dim} W_{2}=b+1$, we can assume that the Young diagram of $\lambda$ has at most $a+1$ rows (otherwise $\mathbb{S}_{\lambda}\left(W_{1}\right)=0$ ) and at most $b+1$ columns (otherwise $\mathbb{S}_{\lambda^{\prime}}\left(W_{2}\right)=0$ ).

By the Pieri formula [Fulton and Harris 1991, (6.8)], for any partition $\lambda$, we have

$$
\mathbb{S}_{\lambda}\left(W_{1}\right) \otimes \operatorname{Sym}^{b-1}\left(W_{1}\right) \cong \bigoplus_{\nu} \mathbb{S}_{\nu}\left(W_{1}\right),
$$

where the sum is over all $v$ whose Young diagram is obtained by adding $b-1$ boxes to the Young diagram of $\lambda$, with no two boxes in the same column. Note also that each $v$ is a partition of $(a b+1)+(b-1)=(a+1) b$. Since $D_{\lambda}$ has $|\lambda|=a b+1$ boxes and fits inside a $(a+1) \times(b+1)$ rectangle, the only way for $v$ to give the trivial representation is for $D_{\lambda}$ to be the Young diagram.

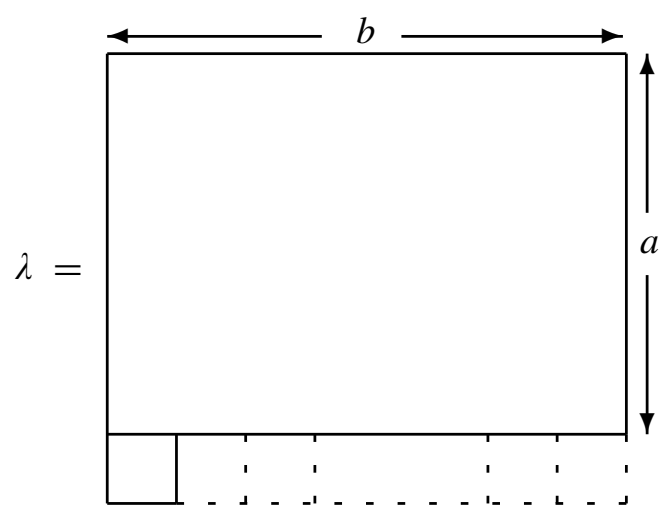

You can see how adding $b-1$ boxes to the bottom row (the dashed boxes in the drawing) gives the trivial representation, since $D_{v}$ is trivial if and only if it consists entirely of columns of height $a+1$.

This shows that the only case when

$$
\mathbb{S}_{\lambda}\left(W_{1}\right) \otimes \operatorname{Sym}^{b-1}\left(W_{1}\right)
$$

contains the trivial representation is when $\lambda=\left(b^{a}, 1\right)$. Hence, $\lambda^{\prime}$ must be

$$
\left(a+1, a^{b-1}\right) .
$$

On the other hand, $\operatorname{Sym}^{a-\beta}\left(W_{2}^{*}\right)$ corresponds to the partition $\left(b^{a-\beta}\right)$ [Fulton and Harris 1991, §15.5, Exercise 15.50], so from the Pieri formula we see that it is impossible to get $\left(b^{a-\beta}\right)$ from the tensor product $\mathbb{S}_{\lambda^{\prime}}\left(W_{2}\right) \otimes \operatorname{Sym}^{\beta-1}\left(W_{2}\right)$ by adding 
$\beta-1$ boxes to $\left(a+1, a^{b-1}\right)$, no two in the same column, and then deleting columns of height $b+1$.

The final step is to prove exactness when $T^{p} \rightarrow T^{p+1}$ is given by

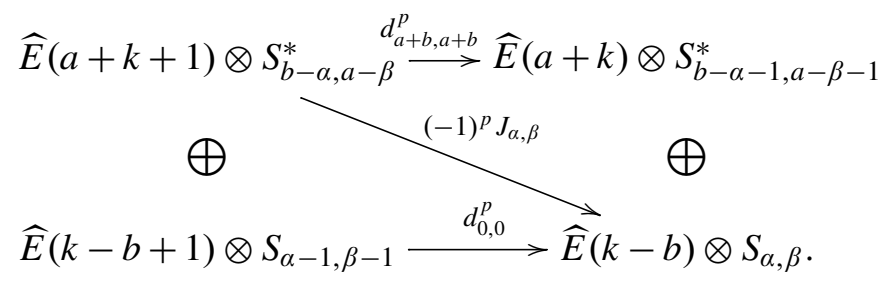

Here, we use the same conventions as in (4-9), except that we now assume that $b-\alpha$ and $a-\beta$ are positive. As before, the shape of the Tate resolution tells us that there are $\operatorname{dim}\left(S_{\alpha-1, \beta-1}\right)$ minimal generators of degree $N^{\prime}-(k-b+1)$ and $\operatorname{dim}\left(S_{b-\alpha, a-\beta}^{*}\right)$ minimal generators of degree $N^{\prime}-(a+k+1)$. The former are taken care of by the known map $d_{0,0}^{p}$, and for the latter, we see that in degree $N^{\prime}-(a+k+1)$, the above diagram becomes

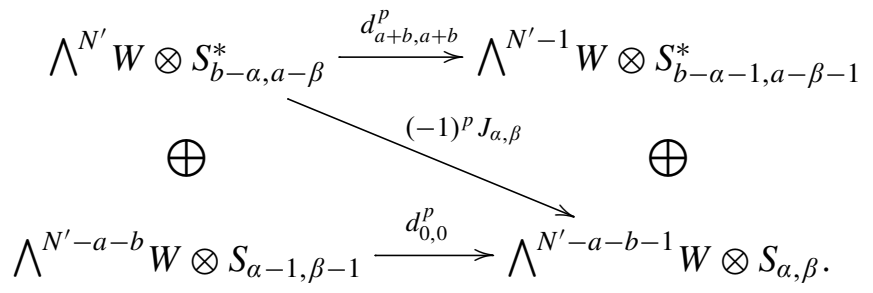

The map $d_{a+b, a+b}^{p}$ is injective since it is dual to the surjective multiplication map $W \otimes S_{b-\alpha-1, a-\beta-1} \rightarrow S_{b-\alpha, a-\beta}$. As in the proof of [Cox 2007, Theorem 1.3], it follows immediately that the map (4-12) is injective on $\bigwedge^{N^{\prime}} W \otimes S_{b-\alpha, a-\beta}^{*}$ and that the images of

$$
\bigwedge^{N^{\prime}} W \otimes S_{b-\alpha, a-\beta}^{*} \quad \text { and } \quad \bigwedge^{N^{\prime}-a-b} W \otimes S_{\alpha-1, \beta-1}
$$

have trivial intersection. This completes the proof of the theorem.

Remark 4.4. In the proof of Section 4.3, we used the relation between the toric Jacobian of $f_{0}, \ldots, f_{a+b} \in S_{1,1}$ and the hyperdeterminants studied in [Gel'fand et al. $1994 ; 1992]$ to prove the equivariance we needed. The theorem implies that certain hyperdeterminants are explicitly encoded into the Tate resolutions considered here. This is another example of the amazing amount of information contained in these resolutions. 


\section{Acknowledgements}

We are very grateful to Jenia Tevelev for discussions about Young diagrams and representation theory. We would like to thank Rob Benedetto for a helpful suggestion in the proof of Section 4.3.

\section{References}

[Bernšteĭn et al. 1978] I. N. Bernšteĭn, I. M. Gel'fand, and S. I. Gel'fand, "Algebraic vector bundles on $\mathbf{P}^{n}$ and problems of linear algebra", Funktsional. Anal. i Prilozhen. 12:3 (1978), 66-67. MR 80c:14010a

[Bondy and Murty 1981] J. A. Bondy and U. S. R. Murty, Graph Theory with Applications, NorthHolland, New York, 1981.

[Cox 1996] D. A. Cox, “Toric residues”, Ark. Mat. 34:1 (1996), 73-96. MR 97e:14062 Zbl 0904. 14029

[Cox 2007] D. A. Cox, "Bezoutians and Tate resolutions", J. Algebra 311:2 (2007), 606-618. MR 2008f:14034 Zbl 1118.14020

[Cox and Materov $\geq 2008]$ D. Cox and E. Materov, "Tate resolutions for products of projective spaces". in preparation.

[Eisenbud 2005] D. Eisenbud, The geometry of syzygies, Grad. Texts in Math. 229, Springer, New York, 2005. MR 2005h:13021 Zbl 1066.14001

[Eisenbud et al. 2003a] D. Eisenbud, G. Fløystad, and F.-O. Schreyer, "Sheaf cohomology and free resolutions over exterior algebras", Trans. Amer. Math. Soc. 355:11 (2003), 4397-4426. MR 2004f: 14031 Zbl 1063.14021

[Eisenbud et al. 2003b] D. Eisenbud, F.-O. Schreyer, and J. Weyman, "Resultants and Chow forms via exterior syzygies”, J. Amer. Math. Soc. 16:3 (2003), 537-579. MR 2004j:14067 Zbl 1069.14019

[Fløystad 2000] G. Fløystad, "Describing coherent sheaves on projective spaces via Koszul dualities", preprint, 2000. arXiv math.AG/0012263

[Fløystad 2004] G. Fløystad, "Exterior algebra resolutions arising from homogeneous bundles", Math. Scand. 94:2 (2004), 191-201. MR 2005e:13016 Zbl 1062.14023

[Fulton and Harris 1991] W. Fulton and J. Harris, Representation theory, Graduate Texts in Mathematics 129, Springer, New York, 1991. MR 93a:20069 Zbl 0744.22001

[Gel' fand et al. 1992] I. M. Gel' fand, M. M. Kapranov, and A. V. Zelevinsky, "Hyperdeterminants", Adv. Math. 96:2 (1992), 226-263. MR 94g:14023 Zbl 0774.15002

[Gel'fand et al. 1994] I. M. Gel'fand, M. M. Kapranov, and A. V. Zelevinsky, Discriminants, resultants, and multidimensional determinants, Mathematics: Theory \& Applications, Birkhäuser, Boston, 1994. MR 95e:14045 Zbl 0827.14036

[Khetan 2003] A. Khetan, "The resultant of an unmixed bivariate system", J. Symbolic Comput. 36:3-4 (2003), 425-442. MR 2004g:13027 Zbl 1068.14070

[Khetan 2005] A. Khetan, "Exact matrix formula for the unmixed resultant in three variables", $J$. Pure Appl. Algebra 198:1-3 (2005), 237-256. MR 2006a:13041 Zbl 1069.14056

[Weyman and Zelevinsky 1994] J. Weyman and A. Zelevinsky, "Determinantal formulas for multigraded resultants”, J. Algebraic Geom. 3:4 (1994), 569-597. MR 95j:14074 Zbl 0816.13007

Communicated by David Eisenbud

Received 2008-01-14 Accepted 2008-05-28 
dac@cs.amherst.edu

materov@math.umass.edu
Department of Mathematics and Computer Science, Amherst College, Amherst, MA 01002-5000, United States www.amherst.edu/ dacox

Department of Mathematics and Statistics, University of Massachusetts, Amherst, MA 01003-9305, United States 


\title{
Constructing simply laced Lie algebras from extremal elements
}

\author{
Jan Draisma and Jos in 't panhuis
}

\begin{abstract}
For any finite graph $\Gamma$ and any field $K$ of characteristic unequal to 2, we construct an algebraic variety $X$ over $K$ whose $K$-points parametrize $K$-Lie algebras generated by extremal elements, corresponding to the vertices of the graph, with prescribed commutation relations, corresponding to the nonedges. After that, we study the case where $\Gamma$ is a connected, simply laced Dynkin diagram of finite or affine type. We prove that $X$ is then an affine space, and that all points in an open dense subset of $X$ parametrize Lie algebras isomorphic to a single fixed Lie algebra. If $\Gamma$ is of affine type, then this fixed Lie algebra is the split finite-dimensional simple Lie algebra corresponding to the associated finite-type Dynkin diagram. This gives a new construction of these Lie algebras, in which they come together with interesting degenerations, corresponding to points outside the open dense subset. Our results may prove useful for recognizing these Lie algebras.
\end{abstract}

\section{Introduction and main results}

An extremal element of a Lie algebra $\mathscr{L}$ over a field $K$ of characteristic unequal to 2 is an element $x \in \mathscr{L}$ for which $[x,[x, \mathscr{L}]] \subseteq K x$. A sandwich element is an $x \in \mathscr{L}$ satisfying the stronger condition $[x,[x, \mathscr{L}]]=0$. The definition of extremal elements in characteristic 2 is more involved, which is one reason for restricting ourselves to characteristics unequal to 2 here. Extremal elements and sandwich elements play important roles in both classical and modern Lie algebra theory. In complex simple Lie algebras, or their split analogues over other fields, extremal elements are precisely the elements that are long-root vectors relative to some maximal torus. Sandwich elements are used in the classification of simple Lie algebras in small characteristics [Premet and Strade 1997]; they occur in the modular Lie algebras of Cartan type, such as the Witt algebras. Sandwich elements were originally introduced in relation with the restricted Burnside problem [Kostrikin 1981]. An

MSC2000: primary 17B20; secondary 14D20, 17B67, $17 \mathrm{~B} 01$.

Keywords: Lie algebras, extremal elements, generators and relations.

Draisma is supported by DIAMANT, an NWO mathematics cluster. In 't panhuis is supported by NWO PhD grant 10002490. 
important insight for the resolution of this problem is the fact that a Lie algebra generated by finitely many sandwich elements is necessarily finite-dimensional. While this fact was first only proved under extra assumptions, in [Zelmanov and Kostrikin 1990] it is proved in full generality. We will use this result in what follows.

The prominence of extremal elements in the work of Kostrikin and Zel'manov and in modular Lie algebra theory led to the natural problem of describing all Lie algebras generated by a fixed number of extremal elements [Cohen et al. 2001; in 't panhuis et al. 2007; Postma 2007; Roozemond 2005].

Example. Suppose that we want to describe all Lie algebras $\mathscr{L}$ generated by two extremal elements $x$ and $y$. Since $[x,[x, y]]$ is a scalar multiple $a x$ of $x$ and $[y,[x, y]]=-[y,[y, x]]$ is a scalar multiple - by of $y, \mathscr{L}$ is spanned by $x, y,[x, y]$. There may be linear dependencies between these elements, but let us assume that they are linearly independent. Then

$a[y, x]=[y,[x,[x, y]]]=[[y, x],[x, y]]+[x,[y,[x, y]]]=0-b[x, y]=b[y, x]$,

and since we have assumed that $[x, y] \neq 0$, we find that $a=b$. Hence threedimensional Lie algebras with a distinguished pair of extremal generators are parametrized by the single number $a$. Moreover, all algebras with $a \neq 0$ are mutually isomorphic and isomorphic to the split simple Lie algebra of type $A_{1}$, while the algebra with $a=0$ is nilpotent and isomorphic to the three-dimensional Heisenberg algebra. This is a prototypical example of our results. The next smallest case of three generators is treated in [Cohen et al. 2001; Zelmanov and Kostrikin 1990] and also by our results below. There the generic Lie algebra is split of type $A_{2}$ and more interesting degenerations exist.

We now generalize and formalize this example to the case of more generators, where we also allow for the flexibility of prescribing that certain generators commute. Thus let $\Gamma$ be a finite simple graph without loops or multiple edges. Let $\Pi$ be the vertex set of $\Gamma$ and denote the neighbor relation by $\sim$. Fixing a field $K$ of characteristic unequal to 2 , we denote by $\mathscr{F}$ the quotient of the free Lie algebra over $K$ generated by $\Pi$ modulo the relations

$$
[x, y]=0 \quad \text { for all } x, y \in \Pi \text { with } x \ngtr y .
$$

So $\mathscr{F}$ depends both on $\Gamma$ and on $K$, but we will not make this dependence explicit in the notation. We write $\mathscr{F}^{*}$ for the space of all $K$-linear functions $\mathscr{F}_{F} \rightarrow K$. For every $f \in\left(\mathscr{F}^{*}\right)^{\Pi}$, also written $\left(f_{x}\right)_{x \in \Pi}$, we denote by $\mathscr{L}(f)$ the quotient of $\mathscr{F}$ by the ideal $\mathscr{T}(f)$ generated by the (infinitely many) elements

$$
[x,[x, y]]-f_{x}(y) x \quad \text { for } x \in \Pi \text { and } y \in \mathscr{F} .
$$


By construction, $\mathscr{L}(f)$ is a Lie algebra generated by extremal elements, corresponding to the vertices of $\Gamma$, which commute when they are not connected in $\Gamma$. The element $f_{x}$ is a parameter needed to express the extremality of $x \in \Pi$. If $\Gamma$ is not connected, then both $\mathscr{F}$ and $\mathscr{L}(f)$ naturally split into direct sums over all connected components of $\Gamma$, so it is no restriction to assume that $\Gamma$ is connected; we will do so throughout this paper.

In the Lie algebra $\mathscr{L}(0)$, the elements of $\Pi$ map to sandwich elements; hence by [Zelmanov and Kostrikin 1990] this Lie algebra is finite-dimensional. For general $f \in\left(\mathscr{F}^{*}\right)^{\Pi}$ it turns out that $\operatorname{dim} \mathscr{L}(f) \leq \operatorname{dim} \mathscr{L}(0)$; see [Cohen et al. 2001] or the proof of Theorem 1 below. It is therefore natural to focus on the Lie algebras $\mathscr{L}(f)$ of the maximal possible dimension $\operatorname{dim} \mathscr{L}(0)$. This leads us to define the set

$$
X:=\left\{f \in\left(\mathscr{F}^{*}\right)^{\Pi} \mid \operatorname{dim} \mathscr{L}(f)=\operatorname{dim} \mathscr{L}(0)\right\},
$$

the parameter space for all maximal-dimensional Lie algebras of the form $\mathscr{L}(f)$.

Example. In the two-generator case above, $\Gamma$ is the graph with two vertices joined by an edge. The sandwich algebra $\mathscr{L}(0)$ is the three-dimensional Heisenberg algebra, and the condition that $\operatorname{dim} \mathscr{L}(f)=3$ corresponds to our assumption above that $x, y,[x, y]$ be linearly independent. This linear independence forced the parameters $a$ and $b$ to be equal. Here $X$ is the affine line with coordinate $a$. All Lie algebras corresponding to points $a \neq 0$ are mutually isomorphic.

Our first main result is that $X$ carries a natural structure of an affine algebraic variety. To specify this structure we note that $\Phi(0)$ is a homogeneous ideal relative to the natural $\mathbb{N}$-grading that $\mathscr{F}$ inherits from the free Lie algebra generated by $\Pi$.

Theorem 1. The set $X$ is naturally the set of $K$-rational points of an affine variety of finite type defined over $K$. This variety can be described as follows. Fix any finite-dimensional homogeneous subspace $V$ of $\mathscr{F}$ such that $V+\mathscr{T}(0)=\mathscr{F}$. Then the restriction map

$$
X \rightarrow\left(V^{*}\right)^{\Pi}, f \mapsto\left(\left.f_{x}\right|_{V}\right)_{x \in \Pi}
$$

maps $X$ injectively onto the set of $K$-rational points of a closed subvariety of $\left(V^{*}\right)^{\Pi}$. This yields a $K$-variety structure on $X$ which is independent of the choice of $V$.

We prove this theorem in Section 2. In Section 3 we first derive some relations between the sandwich algebra $\mathscr{L}(0)$ and the positive part of the complex KacMoody algebra of type $\Gamma$. Then we determine $\mathscr{L}(0)$ explicitly in the case where $\Gamma$ is a simply laced Dynkin diagram of finite or affine type; by this we mean any of the diagrams in Figure 1 without or with vertex 0, respectively. See Theorems 12 and 13. In Section 4 we study the variety $X$. After some observations for general $\Gamma$, we again specialize to the diagrams of Figure 1 . For these we prove that $X$ is an 


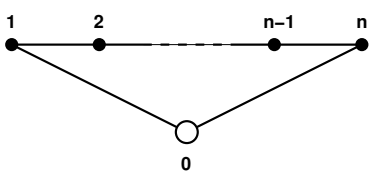

$A_{n}^{(1)}$

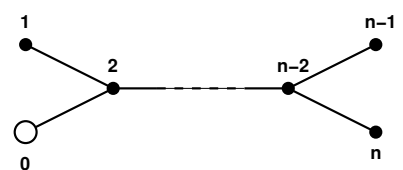

$D_{n}^{(1)}$

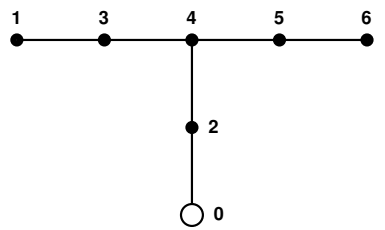

$E_{6}^{(1)}$

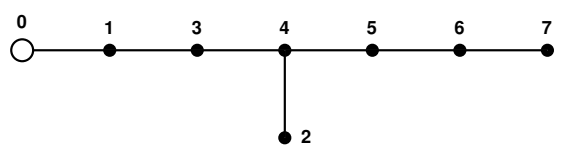

$E_{7}^{(1)}$

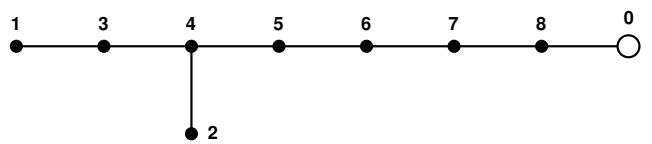

$E_{8}^{(1)}$

Figure 1. The simply laced Dynkin diagrams of affine type. The notation comes from [Kac 1990], and the corresponding finitetype diagrams are obtained by deleting vertex 0 .

affine space, and that for $f$ in an open dense subset of $X$, the Lie algebra $\mathscr{L}(f)$ is isomorphic to a fixed Lie algebra; see Theorems 19 and 22. The latter of these theorems is our second main result, and we paraphrase it here.

Theorem 2. Let $\Gamma$ be any of the simply laced Dynkin diagrams of affine type in Figure 1, let $\Gamma^{0}$ be the finite-type diagram obtained by removing vertex 0 from $\Gamma$, and let $\Sigma$ be the edge set of $\Gamma$. Then $X$ is isomorphic to the affine space of dimension $|\Sigma|+1$ over $K$, and for $f$ in an open dense subset of $X$, the Lie algebra $\mathscr{L}(f)$ is isomorphic to the Chevalley algebra of type $\Gamma^{0}$.

Remark 3. By the Chevalley algebra of type $\Gamma^{0}$ we mean the Lie algebra obtained by tensoring a certain $\mathbb{Z}$-form of the complex simple Lie algebra of type $\Gamma^{0}$ with the field $K$; see Section 3.2 for details. This Lie algebra is often simple, but not always; see [Strade 2004, Chapter 4] and [Seligman 1967].

In Section 5 we will conclude with remarks on applications and related work.

\section{The variety structure of the parameter space}

Recall the notation from Section 1: $\Gamma$ is a connected finite graph without loops or multiple edges, $K$ is a field of characteristic unequal to 2, and $X$ is the set of all $f \in\left(\mathscr{F}^{*}\right)^{\Pi}$ such that $\mathscr{L}(f)$ has the maximal possible dimension, namely that of $\mathscr{L}(0)$. To avoid formulas with many Lie brackets, we write $x_{d} \cdots x_{1}$ for the expression $\left[x_{d},\left[\cdots\left[x_{2}, x_{1}\right] \cdots\right]\right]$. Such an element is called a monomial in the $x_{i}$ of degree $d$. In the proof of Theorem 1 we use the $\mathbb{N}$-grading $\mathscr{F}_{F}=\bigoplus_{d=1}^{\infty} \mathscr{F}_{d}$ of $\mathscr{F}_{F}$, where $\mathscr{F}_{d}$ is the span of all monomials of degree $d$ in the elements of $\Pi$. We also use the following terminology: A subspace $V$ of $\mathscr{F}$ is called homogeneous if it 


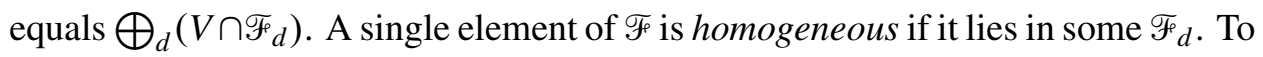
any subspace $V$ of $\mathscr{F}$ we associate the homogeneous subspace gr $V$ of $\mathscr{F}$ spanned by all $v_{d}$ as $v=v_{1}+\cdots+v_{d}$ for $v_{i} \in \mathscr{F}_{i}$ runs through $V$. If $V$ is an ideal, then so is $\operatorname{gr} V$.

Proof of Theorem 1. Let $V$ be a finite-dimensional homogeneous subspace of $\mathscr{F}$ such that $\mathscr{F}=V \oplus \mathscr{I}(0)$; such a subspace exists as $\mathscr{L}(0)$ is finite-dimensional [Cohen et al. 2001; Zelmanov and Kostrikin 1990] and $\mathscr{\Phi}(0)$ is homogeneous. Note that the theorem only requires that $\mathscr{F}=V+\mathscr{(}(0)$; we will argue later why this suffices. Observe that $V$ contains the image of $\Pi$ in $\mathscr{L}$ : the abelian Lie algebra spanned by $\Pi$ is clearly a quotient of $\mathscr{L}(0)$, so the component of $\mathscr{T}(0)$ in degree 1 is trivial. From the shape of the generators (1), it is clear that the homogeneous ideal gr $\mathscr{\Psi}(f)$ associated to $\mathscr{I}(f)$ contains $\mathscr{I}(0)$, so that $\mathscr{F}=V+\mathscr{I}(f)$ for all $f$, and $\mathscr{F}=V \oplus \mathscr{I}(f)$ if and only if $f \in X$. We will argue that the map

$$
\Psi: X \rightarrow\left(V^{*}\right)^{\Pi}, f \mapsto\left(\left.f_{x}\right|_{V}\right)_{x \in \Pi}=:\left.f\right|_{V}
$$

is injective, and that its image is a closed subvariety of $\left(V^{*}\right)^{\Pi}$.

For each $f \in X$, let $\pi_{f}: \mathscr{F}_{P} \rightarrow V$ be the projection onto $V$ along $\Phi(f)$. We prove two slightly technical statements: First, for all $u \in \mathscr{F}$ there exists a polynomial map $P_{u}:\left(V^{*}\right)^{\Pi} \rightarrow V$ such that

$$
P_{u}\left(\left.f\right|_{V}\right)=\pi_{f}(u) \text { for all } f \in X
$$

and second, for $x \in \Pi$ and $u \in \mathscr{F}$ there exists a polynomial $Q_{x, u}:\left(V^{*}\right)^{\Pi} \rightarrow K$ such that

$$
\begin{aligned}
Q_{x, u}\left(\left.f\right|_{V}\right)=f_{x}(u) & \text { for all } f \in X \text { and } \\
Q_{x, u}(h)=h_{x}(u) & \text { if } u \in V \text { and } h \in\left(V^{*}\right)^{\Pi .}
\end{aligned}
$$

We proceed by induction on the degree of $u$ : assume that both statements are true in all degrees less than $d$, and write $u=u_{1}+u_{2}+u_{3}$, where $u_{1}$ has degree less than $d, u_{2} \in V \cap \mathscr{F}_{d}$, and $u_{3} \in \mathscr{I}(0) \cap \mathscr{F}_{d}$. Then $u_{3}$ can be written as a sum of terms of the form $x_{k} \cdots x_{1} x_{1} u^{\prime}$ with $x_{i} \in \Pi$ and $u^{\prime}$ of degree $d-(k+1)<d$. Modulo $\Phi(f)$ for $f \in X$, this term is equal to

$$
f_{x_{1}}\left(u^{\prime}\right) \pi_{f}\left(x_{k} \cdots x_{1}\right)=Q_{x_{1}, u^{\prime}}\left(\left.f\right|_{V}\right) P_{x_{k} \cdots x_{1}}\left(\left.f\right|_{V}\right),
$$

where we used the induction hypothesis for $u^{\prime}$ and $x_{k} \cdots x_{1}$. Hence a $P_{u}$ of the form

$$
P_{u}:=P_{u_{1}}+u_{2}+\text { terms of the form } Q_{x_{1}, u^{\prime}} P_{x_{k} \cdots x_{1}}
$$

has the required property. Similarly, for $x \in \Pi$ and $f \in X$ we have $f_{x}\left(x_{k} \cdots x_{1} x_{1} u^{\prime}\right) x=x x x_{k} \cdots x_{1} x_{1} u^{\prime}=Q_{x_{1}, u^{\prime}}\left(\left.f\right|_{V}\right) Q_{x, x_{k} \cdots x_{1}}\left(\left.f\right|_{V}\right) x \bmod \mathscr{I}(f)$, 
and since $x \notin \mathscr{I}(f)$ we conclude that

$$
f_{x}\left(x_{k} \cdots x_{1} x_{1} u^{\prime}\right)=Q_{x_{1}, u^{\prime}}\left(\left.f\right|_{V}\right) Q_{x, x_{k} \cdots x_{1}}\left(\left.f\right|_{V}\right) .
$$

Hence we may define $Q_{x, u}$ by

$$
Q_{x, u}(h):=Q_{x, u_{1}}(h)+h_{x}\left(u_{2}\right)+\text { terms of the form } Q_{x_{1}, u^{\prime}}(h) Q_{x, x_{k} \cdots x_{1}}(h)
$$

for $h \in\left(V^{*}\right)^{\Pi}$. This shows the existence of $P_{u}$ and $Q_{x, u}$. The injectivity of $\Psi$ is now immediate: any $f \in X$ is determined by its restriction to $V$ by $f_{x}(u)=Q_{x, u}\left(\left.f\right|_{V}\right)$.

We now show that $\operatorname{im}(\Psi)$ is closed. For any tuple $h \in\left(V^{*}\right)^{\Pi}$, one may try to define a Lie algebra structure on $V$ by setting

$$
[u, v]_{h}:=P_{[u, v]}(h) \text { for } u, v \in V .
$$

By construction, if $h=\left.f\right|_{V}$ for some $f \in X$, then this turns $V$ into a Lie algebra isomorphic to $\mathscr{L}(f)$. In this case the Lie bracket has the following two properties:

(i) If $v \in V$ is expressed as a linear combination $\sum_{x_{1}, \ldots, x_{d} \in \Pi} c_{\left(x_{d}, \ldots, x_{1}\right)} x_{d} \cdots x_{1}$ of monomials in the elements of $\Pi$, where the Lie bracket is taken in $\mathscr{F}$, then the expression $\sum_{x_{1}, \ldots, x_{d} \in \Pi} c_{\left(x_{d}, \ldots, x_{1}\right)}\left[x_{d},\left[\cdots\left[x_{2}, x_{1}\right]_{h} \cdots\right]_{h}\right]_{h}$ also equals $v$; and

(ii) $\left[x,[x, u]_{h}\right]_{h}=Q_{x, u}(h) x$ for all $x \in \Pi$ and $u \in V$.

Conversely, suppose that $[\cdot, \cdot]_{h}$ indeed defines a Lie algebra on $V$ satisfying (i) and (ii). Then $\left(V,[\cdot, \cdot]_{h}\right)$ is a Lie algebra of dimension $\operatorname{dim} \mathscr{L}(0)$ that by (i) is generated by the image of $\Pi$, and by (ii) this image consists of extremal elements. Hence there exists an $f \in X$ corresponding to this Lie algebra, and its restriction to $V$ is $h$-indeed, $f_{x}(u)$ is the coefficient of $x$ in $\left[x[x, u]_{h}\right]_{h}$, which is $Q_{x, u}(h)=$ $h_{x}(u)$ for $u \in V$. Finally, all stated conditions on $h$ - the fact that $[\cdot, \cdot]_{h}$ satisfies the Jacobi identity and anticommutativity, together with (i) and (ii) — are closed; here we use the polynomiality of $P_{u}$ and $Q_{x, u}$. This proves that im( $(\Psi)$ is closed.

Now if $U$ is any homogeneous subspace containing $V$, then the restriction map $\Psi^{\prime}: X \rightarrow\left(U^{*}\right)^{\Pi}$ is clearly also injective. Moreover, an $h^{\prime} \in\left(U^{*}\right)^{\Pi}$ lies in the image of this map if and only if $\left.h^{\prime}\right|_{V}$ lies in im $\Psi$ and $h_{x}^{\prime}(u)=Q_{x, u}\left(\left.h^{\prime}\right|_{V}\right)$ for all $u \in U$. Thus $\operatorname{im} \Psi^{\prime}$ is closed, and the maps $\operatorname{im} \Psi^{\prime} \rightarrow \operatorname{im} \Psi,\left.h^{\prime} \mapsto h^{\prime}\right|_{V}$ and $\operatorname{im} \Psi \rightarrow \operatorname{im} \Psi^{\prime}, h \mapsto h^{\prime}$ with $h_{x}^{\prime}(u)=Q_{x, u}(h)$ for $u \in U$ are inverse morphisms between im $\Psi$ and im $\Psi^{\prime}$. Similarly, if $V^{\prime}$ is any other homogeneous vector space complement of $\mathscr{}(0)$ contained in $U$, then the restriction map $\left(U^{*}\right)^{\Pi} \rightarrow\left(\left(V^{\prime}\right)^{*}\right)^{\Pi}$ induces an isomorphism between the images of $X$ in these spaces. This shows that the variety structure of $X$ does not depend on the choice of $V$. Finally, all morphisms indicated here are defined over $K$. We conclude that we have a $K$ variety structure on $X$ that is independent of the choice of $V$.

The type of reasoning in this proof will return in Section 4: in the case where $\Gamma$ is a Dynkin diagram, we will show that for $f \in X$ the restriction $\left.f\right|_{V}$ actually depends 
polynomially on even fewer values of the $f_{x}$, thus embedding $X$ into smaller affine spaces. That these embeddings are closed can be proved exactly as we did above.

\section{The sandwich algebra}

For now, $\Gamma$ is an arbitrary finite graph (not necessarily a Dynkin diagram). The Lie algebra $\mathscr{L}(0)$ is the so-called sandwich algebra corresponding to $\Gamma$. It is a finite-dimensional nilpotent Lie algebra, and carries an $\mathbb{N}^{\Pi}$-grading defined as follows. The weight of a word $\left(x_{d}, \ldots, x_{1}\right)$ over $\Pi$ is the element $\mu \in \mathbb{N}^{\Pi}$ whose $x$-coordinate equals

$$
\left|\left\{i \in\{1, \ldots, d\} \mid x_{i}=x\right\}\right|
$$

for all $x \in \Pi$. For such a word the corresponding monomial $x_{d} \cdots x_{1}$ lives in the free Lie algebra on $\Pi$, but we use the same notation for its images in $\mathscr{F}$ and $\mathscr{L}(f)$ when this does not lead to any confusion. We will sometimes say that a monomial $x_{d} \cdots x_{1} \in \mathscr{L}(0)$ has weight $\mu$, by which we mean that the word $\left(x_{d}, \ldots, x_{1}\right)$ has weight $\mu$ - the monomial $x_{d} \cdots x_{1}$ itself might be 0 . Now the free Lie algebra is graded by weight, and this grading refines the grading by degree. Like the grading by degree, the grading by weight is inherited by $\mathscr{L}(0)$ as all relations defining $\mathscr{L}(0)$ are monomials. We write $\mathscr{L}(0)_{\mu}$ for the space of weight $\mu \in \mathbb{N}^{\Pi}$ and call $\operatorname{dim} \mathscr{L}(0)_{\mu}$ the multiplicity of $\mu$.

For $x \in \Pi$, let $\alpha_{x}$ be the element with a 1 on position $x$ and zeros elsewhere; that is, $\alpha_{x}$ is the weight of the word $(x)$. We define a symmetric $\mathbb{Z}$-bilinear form $\langle\cdot, \cdot\rangle$ on $\mathbb{Z}^{\Pi}$ by its values on the standard basis: for $x, y \in \Pi$ we set

$$
\left\langle\alpha_{x}, \alpha_{y}\right\rangle:=\left\{\begin{aligned}
2 & \text { if } x=y, \\
-1 & \text { if } x \sim y, \text { and } \\
0 & \text { otherwise. }
\end{aligned}\right.
$$

The matrix $A:=\left(\left\langle\alpha_{x}, \alpha_{y}\right\rangle\right)_{x, y \in \Pi}$ is called the Cartan matrix of $\Gamma$. The height of an element of $\mathbb{Z}^{\Pi}$ is by definition the sum of the coefficients of the $\alpha_{x}$ in it for $x \in \Pi$.

In what follows we often need to show that certain monomials $x_{d} \cdots x_{1}$ are zero in $\mathscr{L}(0)$. Lemmas 6-10 show how the bilinear form comes into play. But first we recall an elementary property of sandwich elements, to which they owe their name.

Lemma 4. Let $x$ be a sandwich element in a Lie algebra $\mathscr{L}$, and let $y, z \in \mathscr{L}$ be arbitrary. Then $x y x z=0$.

Proof. We have

$$
\begin{aligned}
x y x z & =[x, y] x z+y x x z=[x, y] x z=-[x, x y] z+x[x, y] z \\
& =z x x y+x[x, y] z=x[x, y] z=x x y z-x y x z=-x y x z .
\end{aligned}
$$


Remark 5. Note that we have used here that the characteristic is not 2 . In the case of characteristic 2, this lemma should be taken as part of the definition of a sandwich element [Cohen and Ivanyos 2006].

Lemma 6. Let $w=\left(x_{d}, x_{d-1}, \ldots, x_{1}\right)$ be a word over $\Pi$, and let $x \in \Pi$. Let $x_{i}$ and $x_{j}$ be consecutive occurrences of $x$ in $w$ (that is, $i>j, x_{i}=x_{j}=x$, and $x_{k} \neq x$ for all $k$ strictly between $i$ and $j$ ). Suppose that the letters in $w$ strictly between $x_{i}$ and $x_{j}$ contain at most 1 occurrence of a $\Gamma$-neighbor of $x$, that is, the set $\left\{k \in\{j+1, \ldots, i-1\} \mid x_{k} \sim x\right\}$ has cardinality at most 1 . Then $x_{d} x_{d-1} \cdots x_{1}$ is 0 in $\mathscr{L}(0)$.

Proof. Set $z:=x_{d} x_{d-1} \cdots x_{1}$. First, using the fact that on $\mathscr{F}$ the linear map $\operatorname{ad}(x)$ commutes with $\operatorname{ad}(y)$ for any $y \in \Pi$ with $x \neq y$, we can move $x_{i}$ in $z$ to the right until it is directly to the left of either $x_{j}$ or the unique $x_{k} \sim x$ between $x_{i}$ and $x_{j}$, so we may assume that this was already the case to begin with.

If $i=j+1$ then either $j=1$ and $z$ is zero by anticommutativity, or $j>1$ and the monomial $x_{i} x_{j} x_{j-1} \cdots x_{1}=x x x_{j-1} \cdots x_{1}$ is zero by the sandwich property of $x$.

Suppose, on the other hand, that $x_{i} \sim x_{i-1}$. Then if $j=1$ and $i-1>2$, the monomial $z$ is zero since $x_{2} x_{1}$ is - indeed, $x_{2} \not x_{1}$. On the other hand, if $i-1=2$ or $j>1$, then we can move $x_{j}$ in $z$ to the left until it is directly to the right of $x_{i-1}$. So again, we may assume that it was there from the beginning. But now

$$
x_{i} x_{i-1} x_{j} x_{j-1} \cdots x_{1}=x x_{i-1} x x_{j-1} \cdots x_{1} \text {. }
$$

If $j>1$, then this monomial equals zero by Lemma 4 ; and if $j=1$, then it is zero by the sandwich property of $x$.

Lemma 7. Let $\left(x_{d}, x_{d-1}, \ldots, x_{1}\right)$ be a word with $d \geq 2$ over $\Pi$, and suppose that the weight $\mu$ of $\left(x_{d-1}, x_{d-2}, \ldots, x_{1}\right)$ satisfies $\left\langle\alpha_{x_{d}}, \mu\right\rangle \geq 0$. Then $x_{d} x_{d-1} \cdots x_{1}=0$ in $\mathscr{L}(0)$.

Proof. Set $w:=\left(x_{d}, x_{d-1}, \ldots, x_{1}\right)$ and $z:=x_{d} x_{d-1} \cdots x_{1}$. The condition on the bilinear form can be written as

$$
2\left|\left\{j \in\{1, \ldots, d-1\} \mid x_{j}=x_{d}\right\}\right| \geq\left|\left\{j \in\{1, \ldots, d-1\} \mid x_{j} \sim x_{d}\right\}\right| .
$$

First we note that if the right side is 0 , then $z$ is trivially zero: then all $x_{i}$ with $i<d$ commute with $x_{d}$, and there at least $d-1 \geq 1$ such factors. So we may assume that the right side is positive, and hence so is the left side.

Let the set in the left side of this inequality consist of the indices $i_{m}>i_{m-1}>$ $\cdots>i_{1}$. By the above, $m$ is positive. In the word $w$ there are $m$ pairs $(i, j)$ satisfying the conditions of Lemma 6 with $x=x_{d}$, namely, $\left(d, i_{m}\right),\left(i_{m}, i_{m-1}\right), \ldots$, $\left(i_{2}, i_{1}\right)$. Now if for some such $(i, j)$ there are less than two $\Gamma$-neighbors of $x_{d}$ in the interval between $x_{i}$ and $x_{j}$, then $z=0$ by Lemma 6 . So we may assume that each of these $m$ intervals contains at least two $\Gamma$-neighbors of $x_{d}$. But then, by the 
inequality above, these exhaust all $\Gamma$-neighbors of $x_{d}$ in $w$, so in particular there are exactly $2 \Gamma$-neighbors of $x_{d}$ between $x_{i_{2}}$ and $x_{i_{1}}$, and none to the right of $x_{i_{1}}$. Now if $i_{1}>1$, then $z$ is zero because $x_{i_{1}}$ commutes with everything to the right of it. Hence assume that $i_{1}=1$, and note that $i_{2} \geq 4$. If $x_{2} \nsucc x_{1}=x_{i_{1}}$, then again $z$ is trivially 0 , so assume that $x_{2}$ is a $\Gamma$-neighbor of $x_{d}=x_{1}$. Then we have

$$
x_{i_{2}} \cdots x_{3} x_{2} x_{1}=-x_{i_{2}} \cdots x_{3} x_{1} x_{2}
$$

but in the monomial on the right there is only one $\Gamma$-neighbor of $x_{d}$ between $x_{i_{2}}$ and $x_{1}$ - hence it is zero by Lemma 6 .

Lemma 8. Let $x \in \Pi$ and $\lambda \in \mathbb{N}^{\Pi}$ satisfy $\left\langle\alpha_{x}, \lambda\right\rangle=-1$. Then $\mathscr{L}(0)_{\alpha_{x}+\lambda}=$ $\left[x, \mathscr{L}(0)_{\lambda}\right]$.

Proof. Let $w=\left(x_{d}, \ldots, x_{1}\right)$ be a word over $\Pi$ of weight $\alpha_{x}+\lambda$. We show that in $\mathscr{L}(0)$ the monomial $z:=x_{d} \cdots x_{1}$ is a scalar multiple of some monomial of the form $x z^{\prime}$, where $z^{\prime}$ is a monomial of weight $\lambda$. Obviously, $x$ occurs in $w$; let $k$ be maximal with $x_{k}=x$. If $k=1$, then we may interchange $x_{k}=x_{1}$ and $x_{k+1}=x_{2}$ in $z$ at the cost of a minus sign (note that $d \geq 2$ ), so we may assume that $k \geq 2$.

Suppose first that there occur $\Gamma$-neighbors of $x=x_{k}$ to the left of $x_{k}$ in $w$. We claim that then $z=0$. Indeed, let $\mu$ and $v$ be the weights of $\left(x_{k-1}, \ldots, x_{1}\right)$ and $\left(x_{d}, \ldots, x_{k+1}\right)$, respectively. Then we have

$$
\left\langle\alpha_{x}, \mu\right\rangle=\left\langle\alpha_{x}, \lambda\right\rangle-\left\langle\alpha_{x}, \nu\right\rangle=-1-\left\langle\alpha_{x}, v\right\rangle \geq 0,
$$

where in the last inequality we use that there are occurrences of neighbors of $x_{k}$, but none of $x_{k}$ itself, in the word $\left(x_{d}, \ldots, x_{k+1}\right)$. Now we find $x_{k} \cdots x_{1}=0$ by Lemma 7 (note that $k \geq 2$ ); hence $z=0$ as claimed.

So we can assume that there are no $\Gamma$-neighbors of $x_{k}$ to the left of $x_{k}$ in $w$. Then we may move $x_{k}$ in $z$ all the way to the left; hence $z$ is indeed equal to $x z^{\prime}$ for some monomial $z^{\prime}$ of weight $\lambda$.

3.1. Relation with the root system of the Kac-Moody algebra. Recall the definition of the Kac-Moody algebra $\mathfrak{g}_{\mathrm{KM}}$ over $\mathbb{C}$ corresponding to $\Gamma$ : it is the Lie algebra generated by $3 \cdot|\Pi|$ generators, denoted $E_{x}, H_{x}, F_{x}$ for $x \in \Pi$, modulo the relations

$$
\begin{aligned}
& H_{x} H_{y}=0, \quad E_{x} F_{x}=H_{x}, \\
& H_{x} E_{y}=\left\langle\alpha_{x}, \alpha_{y}\right\rangle E_{y}, \quad H_{x} F_{y}=-\left\langle\alpha_{x}, \alpha_{y}\right\rangle F_{y} \text {; } \\
& \text { and, for } x \neq y, E_{x} F_{y}=0 \text {, } \\
& \operatorname{ad}\left(E_{x}\right)^{1-\left\langle\alpha_{x}, \alpha_{y}\right\rangle} E_{y}=0 \text {, } \\
& \operatorname{ad}\left(F_{x}\right)^{1-\left\langle\alpha_{x}, \alpha_{y}\right\rangle} F_{y}=0 .
\end{aligned}
$$

Endow $\mathfrak{g}_{\mathrm{KM}}$ with the $\mathbb{Z}^{\Pi}$-grading in which $E_{x}, H_{x}, F_{x}$ have weights $\alpha_{x}, 0,-\alpha_{x}$, respectively. Let $\Phi:=\left\{\beta \in \mathbb{Z}^{\Pi} \backslash\{0\} \mid\left(\mathfrak{g}_{\mathrm{KM}}\right)_{\beta} \neq 0\right\}$ be the root system of $\mathfrak{g}_{\mathrm{KM}}$; it is 
equal to the disjoint union of its subsets $\Phi_{ \pm}:=\Phi \bigcap( \pm \mathbb{N})^{\Pi}$ and contains the simple roots $\alpha_{x}$ for $x \in \Pi$; we refer to [Kac 1990] for the theory of Kac-Moody algebras. In what follows we will compare the multiplicities of weights in the $K$-algebra $\mathscr{L}(0)$ and the $\mathbb{C}$-algebra $\mathfrak{g}_{\mathrm{KM}}$.

Lemma 9. For $\lambda \in \mathbb{N}^{\Pi} \backslash \Phi_{+}$, we have $\mathscr{L}(0)_{\lambda}=0$.

Proof. We proceed by induction on the height of $\lambda$. The proposition is trivially true for $\lambda$ of height 1 . Suppose now that it is true for height $d-1 \geq 1$, and consider a word $w=\left(x_{d}, x_{d-1}, \ldots, x_{1}\right)$ of weight $\lambda \notin \Phi_{+}$.

Set $\mu:=\lambda-\alpha_{x_{d}}$. If $\mu \notin \Phi_{+}$, then $x_{d-1} \cdots x_{1}=0$ by the induction hypothesis, so we may assume that $\mu \in \Phi_{+}$. This together with $\mu+\alpha_{x_{d}} \notin \Phi_{+}$implies (by elementary $\mathfrak{s l}_{2}$-theory in $\mathfrak{g}_{\mathrm{KM}}$ ) that $\left\langle\alpha_{x_{d}}, \mu\right\rangle \geq 0$. Now Lemma 7 shows that $x_{d} \cdots x_{1}=0$.

For another relation between weight multiplicities in $\mathscr{L}(0)$ and $\mathfrak{g}_{\mathrm{KM}}$, recall that a root in $\Phi$ is called real if it is in the orbit of some simple root under the Weyl group $W$ of $\mathfrak{g}_{\mathrm{KM}}$. In that case it has multiplicity 1 in $\mathfrak{g}_{\mathrm{KM}}$. We now call a root $\beta \in \Phi_{+}$very real - this is nonstandard terminology - if it can be written as $\beta=$ $\alpha_{x_{d}}+\cdots+\alpha_{x_{1}}$, for some $x_{1}, \ldots, x_{d} \in \Pi$, such that for all $i=2, \ldots, d$ we have

$$
\left\langle\alpha_{x_{i}}, \alpha_{x_{i-1}}+\cdots+\alpha_{x_{1}}\right\rangle=-1 .
$$

(This implies that $\beta=s_{x_{d}} \cdots s_{x_{2}} \alpha_{x_{1}}$, where the $s_{x}$ are the fundamental reflections corresponding to the $x \in \Pi$.)

Lemma 10. Any very real $\beta \in \Phi_{+}$has multiplicity at most 1 in $\mathscr{L}(0)$.

Proof. This follows by induction on the height of $\beta$, using Lemma 8 for the induction step.

3.2. Simply laced Dynkin diagram of finite type. In this section we assume that $\Gamma$ is a Dynkin diagram of finite type, that is, one of the diagrams in Figure 1 with vertex 0 removed. Then $\mathfrak{g}_{\mathrm{KM}}$ is a finite-dimensional simple Lie algebra over $\mathbb{C}$. Now $\mathfrak{g}_{\mathrm{KM}}$ has a Chevalley basis [Carter 1972, Section 4.2]. This basis consists of the images of the $H_{x}$ and one vector $E_{\alpha} \in\left(\mathfrak{g}_{\mathrm{KM}}\right)_{\alpha}$ for every root $\alpha \in \Phi$, where $E_{\alpha_{x}}$ and $E_{-\alpha_{x}}$ may be taken as $E_{x}$ and $F_{x}$, respectively. An important property that we will need is that $\left[E_{\alpha}, E_{\beta}\right]= \pm E_{\alpha+\beta}$ for all roots $\alpha$ and $\beta$ such that $\alpha+\beta$ is a root; here we use that the $p$ in [Carter 1972, Theorem 4.2.1] is 0 in the simply laced case. The Chevalley basis spans a $\mathbb{Z}$-subalgebra of $\mathfrak{g}_{\mathrm{KM}}$. Let $\mathfrak{g}$ be the $K$-algebra obtained by tensoring this $\mathbb{Z}$-form with $K$, and let $E_{x}^{0}, H_{x}^{0}, F_{x}^{0}$ be the images in $\mathfrak{g}$ of $E_{x}, H_{x}, F_{x}$. The Lie algebra $\mathfrak{g}$ has a triangular decomposition

$$
\mathfrak{g}=\mathfrak{n}_{-} \oplus \mathfrak{h} \oplus \mathfrak{n}_{+},
$$

where $\mathfrak{n}_{ \pm}:=\bigoplus_{\beta \in \Phi_{ \pm}} \mathfrak{g}_{\beta}$. We will refer to $\mathfrak{g}$ as the Chevalley algebra of type $\Gamma$. 
Remark 11. One can also define $\mathfrak{g}$ as the Lie algebra of the split simply connected algebraic group over $K$ of type $\Gamma$.

Theorem 12. Let $\Gamma$ be a simply laced Dynkin diagram of finite type, obtained from a diagram in Figure 1 by removing vertex 0 . Let $\mathfrak{g}$ be the corresponding Chevalley algebra over the field $K$ of characteristic unequal to 2 , and let $\mathfrak{n}_{+}$be the subalgebra generated by the $E_{x}^{0}$. Then the map sending $x \in \Pi$ to $E_{x}^{0}$ induces a (necessarily unique) isomorphism $\mathscr{L}(0) \rightarrow \mathfrak{n}_{+}$.

In the proof of this theorem we use the following well-known facts about simply laced Kac-Moody algebras of finite type: first, $\langle\cdot, \cdot\rangle$ only takes the values $-1,0,1,2$ on $\Phi_{+} \times \Phi_{+}$, and second, all roots in $\Phi_{+}$are very real.

Proof of Theorem 12. To prove the existence of a homomorphism $\pi$ sending $x$ to $E_{x}^{0}$, we verify that the relations defining $\mathscr{L}(0)$ hold in $\mathfrak{n}_{+}$. That is, we have to prove that

$$
\begin{aligned}
{\left[E_{x}^{0}, E_{y}^{0}\right]=0 } & \text { for all } x, y \in \Pi \text { with } x \ngtr y, \\
\operatorname{ad}\left(E_{x}^{0}\right)^{2} z=0 & \text { for all } x \in \Pi \text { and all } z \in \mathfrak{n}_{+} .
\end{aligned}
$$

The first statement is immediate from the relations defining $\mathfrak{g}_{\mathrm{KM}}$. For the second relation, if $z \in \mathfrak{n}_{+}$is a root vector with $\operatorname{root} \beta \in \Phi_{+}$, then $\left\langle\beta, \alpha_{x}\right\rangle \geq-1$ by the above, so that $\left\langle\beta+2 \alpha_{x}, \alpha_{x}\right\rangle \geq 3$, and therefore $\beta+2 \alpha_{x} \notin \Phi_{+}$, so that $\operatorname{ad}\left(E_{x}\right)^{2} z=0$. As root vectors span $\mathfrak{n}_{+}$, we have proved the existence of $\pi$; uniqueness is obvious.

Now we have to show that $\pi$ is an isomorphism. It is surjective since $\mathfrak{n}_{+}$is generated by the $E_{x}^{0}$; this follows from the properties of the Chevalley basis in Section 3.2. Hence it suffices to prove that $\operatorname{dim} \mathscr{L}(0) \leq \operatorname{dim} \mathfrak{n}_{+}$. But by Lemmas 9 and 10 and the fact that all roots are very real, we have $\mathscr{L}(0)_{\mu}=0$ for all $\mu \notin \Phi_{+}$ and $\operatorname{dim} \mathscr{L}(0)_{\beta} \leq \operatorname{dim} \mathfrak{g}_{\beta}$ for all $\beta \in \Phi_{+}$. This concludes the proof.

3.3. Simply laced Dynkin diagrams of affine type. Suppose now that $\Gamma$ is a simply laced Dynkin diagram of affine type from Figure 1. Recall that the Cartan matrix $A$ has a one-dimensional kernel spanned by a unique primitive vector $\delta \in \mathbb{N} \Pi$. Here primitive means that the greatest common divisor of the coefficients of $\delta$ on the standard basis is 1 ; indeed, there always exists a vertex $x_{0} \in \Pi$ (labeled 0 in Figure 1) with coefficient 1 in $\delta$, and all such vertices form an $\operatorname{Aut}(\Gamma)$-orbit. For later use, we let $h$ be the Coxeter number, which is the height of $\delta$.

Let $\Pi^{0}:=\Pi \backslash\left\{x_{0}\right\}$. Write $\Gamma^{0}$ for the induced subgraph on $\Pi^{0}$ (which is a Dynkin diagram of finite type) and $\Phi^{0}$ for the root system of the Chevalley algebra $\mathfrak{g}$ of type $\Gamma^{0}$ defined in Section 3.2. This root system lives in the space $\mathbb{Z}^{\Pi^{0}}$, which we identify with the elements of $\mathbb{Z}^{\Pi}$ that are zero on $x_{0}$. Retain the notation $\mathfrak{n}_{ \pm} \subseteq \mathfrak{g}$ from Section 3.2. Consider the semidirect product $\mathfrak{u}:=\mathfrak{n}_{+} \ltimes \mathfrak{g} / \mathfrak{n}_{+}$, where the second summand is endowed with the trivial Lie bracket and the natural $\mathfrak{n}_{+^{-}}$ module structure. This $\mathfrak{u}$ is clearly a nilpotent Lie algebra; we will prove that it 
is isomorphic to $\mathscr{L}(0)$. In our proof we use the following $\mathbb{Z}^{\Pi}$-grading of $\mathfrak{u}$ : the root spaces in $\mathfrak{n}_{+}$have their usual weight in $\Phi_{+}^{0} \subseteq \mathbb{Z}^{\Pi^{0}}$, while the image of $\mathfrak{g}_{\beta}$ in $\mathfrak{g} / \mathfrak{n}_{+} \subseteq \mathfrak{u}$ for $\beta \in\{0\} \cup \Phi_{-}^{0}$ has weight $\delta+\beta$. Thus the set of all weights occurring in $\mathfrak{u}$ is

$$
\Theta:=\Phi_{+}^{0} \bigcup\left\{\delta+\beta \mid \beta \in \Phi_{-}^{0}\right\} \bigcup\{\delta\} .
$$

Theorem 13. Let $\Gamma$ be a simply laced Dynkin diagram of affine type from Figure 1, let $\Gamma^{0}$ be the subdiagram of finite type obtained by removing vertex 0 , and let $\mathfrak{g}$ be the Chevalley algebra of type $\Gamma^{0}$ over a field of characteristic unequal to 2 . For $x \in \Pi^{0}$, let $E_{x}^{0} \in \mathfrak{n}_{+}$be the element of the Chevalley basis of $\mathfrak{g}$ with simple root $\alpha_{x}$, and for the lowest root $\theta \in \Phi_{-}^{0}$, let $E_{\theta}^{0} \in \mathfrak{g} / \mathfrak{n}_{+}$be the image of the element in the Chevalley basis of weight $\theta$. Then the map sending $x \in \Pi^{0}$ to $E_{x}^{0}$ and $x_{0}$ to $E_{\theta}^{0}$ induces a $\mathbb{Z}^{\Pi}$-graded isomorphism $\mathscr{L}(0) \rightarrow \mathfrak{n}_{+} \ltimes \mathfrak{g} / \mathfrak{n}_{+}$of Lie algebras.

Remark 14. Over $\mathbb{C}$ one can argue directly in the Kac-Moody algebra $\mathfrak{g}_{\mathrm{KM}}$. Then $\mathscr{L}(0)$ is also the quotient of the positive nilpotent subalgebra of $\mathfrak{g}_{\mathrm{KM}}$ by the root spaces with roots of height larger than the Coxeter number $h$. In the proof one uses the root multiplicities of [Kac 1990, Proposition 6.3]. One might also pursue this approach in positive characteristic using the results of [Billig 1990], but we have chosen to avoid defining the Kac-Moody algebra in arbitrary characteristic and use the Chevalley basis instead.

Proof of Theorem 13. The proof is close to that of Theorem 12. We start by verifying that the relations defining $\mathscr{L}(0)$ hold in $\mathfrak{u}=\mathfrak{n}_{+} \ltimes \mathfrak{g} / \mathfrak{n}_{+}$. First, $E_{x}^{0}$ and $E_{y}^{0}$ with $x, y \in \Pi^{0}$ commute when they are not connected in $\Gamma$; this follows from the defining equations of $\mathfrak{g}_{\mathrm{KM}}$. Second, $E_{x}^{0}$ and $E_{\theta}^{0}$ commute if $x \in \Pi^{0}$ is not connected to $x_{0}$, as $\theta+\alpha_{x}$ is then not in $\Phi^{0}$. Third, each $E_{x}^{0}$ is a sandwich element in $\mathfrak{u}$ : for its action on $\mathfrak{n}_{+}$, this follows as in the proof of Theorem 12, and for its action on $\mathfrak{g} / \mathfrak{n}_{+}$, it follows from the fact that $\operatorname{ad}\left(E_{x}^{0}\right)^{2} \mathfrak{g} \subseteq K E_{x}^{0} \subseteq \mathfrak{n}_{+}$. Fourth, $E_{\theta}^{0}$ is a sandwich element as $\operatorname{ad}\left(E_{\theta}^{0}\right)$ maps $\mathfrak{u}$ into $\mathfrak{g} / \mathfrak{n}_{+}$, which has trivial multiplication. This shows the existence of a homomorphism $\pi: \mathscr{L}(0) \rightarrow \mathfrak{u}$. Moreover $\pi$ is graded; in particular, the weight of $E_{\theta}^{0}$ is $\delta+\theta=\alpha_{x_{0}}$.

The $E_{x}^{0}$ generate $\mathfrak{n}_{+}$, and $E_{\theta}^{0}$ generates the $\mathfrak{n}_{+}$-module $\mathfrak{g} / \mathfrak{n}_{+}$. These statements follow from properties of the Chevalley basis in Section 3.2, and imply that $\pi$ is surjective. So we need only show that $\operatorname{dim} \mathscr{L}(0) \leq \operatorname{dim} \mathfrak{u}$; we prove this for each weight in $\Theta$.

First, the roots in $\Phi^{0}$ are very real, so their multiplicities in $\mathscr{L}(0)$ are at most 1 by Lemma 10. Second, we claim that all roots of the form $\delta+\beta$ with $\beta \in \Phi_{-}^{0}$ are also very real. This follows by induction on the height of $\beta$ : For $\beta=\theta$ it is clear since $\delta+\theta=\alpha_{x_{0}}$. For $\beta \neq \theta$ it is well known that there exists an $x \in \Pi^{0}$ such that $\left\langle\alpha_{x}, \beta\right\rangle=1$. Then we have $\delta+\beta=\left(\delta+\beta-\alpha_{x}\right)+\alpha_{x}$, where $\delta+\beta-\alpha_{x} \in \Theta$ and $\left\langle\alpha, \delta+\beta-\alpha_{x}\right\rangle=0+1-2=-1$; here we use that $\delta$ is in the radical of the 
form $\langle\cdot, \cdot\rangle$. By induction, $\delta+\beta-\alpha_{x}$ is very real; hence so is $\delta+\beta$. This shows that also the roots of the form $\delta+\beta$ with $\beta \in \Phi_{-}^{0}$ have multiplicity at most 1 in $\mathscr{L}(0)$, again by Lemma 10 .

Next we show that $\delta$ has multiplicity at most $\left|\Pi^{0}\right|=\operatorname{dim} \mathfrak{h}$ in $\mathscr{L}(0)$. Indeed, we claim that $\mathscr{L}(0)_{\delta}$ is contained in $\sum_{x \in \Pi^{0}}\left[x, \mathscr{L}(0)_{\delta-\alpha_{x}}\right]$. Then, by the above, each of the summands has dimension at most 1 , and we are done. The claim is true almost by definition: any monomial of weight $\delta$ must start with some $x \in \Pi$, so we need only show that monomials starting with $x_{0}$ are already contained in the sum above. Consider any monomial $z:=x_{d} \cdots x_{1}$ of weight $\delta$, where $x_{d}=x_{0}$. As the coefficient of $x_{0}$ in $\delta$ is 1 , none of the $x_{i}$ with $i<d$ is equal to $x_{0}$. But then an elementary application of the Jacobi identity and induction shows that $z$ is a linear combination of monomials that do not start with $x_{0}$.

Finally, we have to show that $\mathscr{L}(0)_{\mu}$ is 0 for $\mu \in \Phi_{+} \backslash \Theta$ (we already have $\mathscr{L}(0)_{\mu}=0$ for $\mu \notin \Phi_{+}$by Lemma 9). But Lemma 7 and the fact that $\left\langle\alpha_{x}, \delta\right\rangle=0$ for all $x \in \Pi$ together imply that $\left[x, \mathscr{L}(0)_{\delta}\right]=0$ for all $x \in \Pi$. So it suffices to show that if $\mu \in \Phi_{+}$is not in $\Theta$, then " $\mu$ can only be reached through $\delta$." More precisely, if $\left(x_{d}, \ldots, x_{1}\right)$ is any word over $\Pi$ such that $\sum_{j=1}^{d} \alpha_{x_{j}}=\mu$ and $\mu_{i}:=\sum_{j=1}^{i} \alpha_{x_{j}} \in \Phi_{+}$for all $i=1, \ldots, d$, then there exists an $i$ such that $\mu_{i}=\delta$. But this follows immediately from the fact that $\delta$ is the only root of height $h$ [Kac 1990, Proposition 6.3]. We find that every monomial corresponding to such a word is zero, and this concludes the proof of the theorem.

\section{The parameter space and generic Lie algebras}

So far we have only considered the Lie algebras $\mathscr{L}(0)$. Now we will be concerned with the variety $X$ of all parameters $f \in\left(\mathscr{F}^{*}\right)^{\Pi}$ for which $\operatorname{dim} \mathscr{L}(f)=\operatorname{dim} \mathscr{L}(0)$. We collect some tools for determining $X$ in the case of simply laced Dynkin diagrams.

4.1. Scaling. First let $\Gamma$ be arbitrary again, not necessarily a Dynkin diagram. Scaling of the generators $x_{i}$ has an effect on $X$ : Given $t=\left(t_{x}\right)_{x \in \Pi}$ in the torus $T:=\left(K^{*}\right)^{\Pi}$, there is a unique automorphism of $\mathscr{F}$ that sends $x \in \Pi$ to $t_{x} x$. This gives an action of $T$ on $\mathscr{F}$, and we endow $\mathscr{F}^{*}$ with the contragredient action. Finally, we obtain an action of $T$ on $X$ by

$$
(t f)_{x}(y):=t_{x}^{-1} f_{x}\left(t^{-1} y\right) \quad \text { for all } t \in T, f \in X, x \in \Pi \text {, and } y \in \mathscr{F} .
$$

Indeed, note that with this definition the automorphism of $\mathscr{F}$ induced by $t$ sends $x x y-f_{x}(y) x \in \mathscr{F}$ to

$$
\begin{aligned}
(t x)(t x)(t y)-f_{x}(y) t x & =t_{x}^{2}\left(x x(t y)-t_{x}^{-1} f_{x}(y) x\right) \\
& =t_{x}^{2}\left(x x(t y)-t_{x}^{-1} f_{x}\left(t^{-1}(t y)\right) x\right) \\
& =t_{x}^{2}\left(x x(t y)-(t f)_{x}(t y) x\right),
\end{aligned}
$$


and hence the ideal $\mathscr{I}(f)$ defining $\mathscr{L}(f)$ to $\mathscr{I}(t f)$. Therefore, this automorphism of $\mathscr{F}$ induces an isomorphism $\mathscr{L}(f) \rightarrow L(t f, \Gamma)$.

This scaling action of $T$ on $X$ will make things very easy in the case of simply laced Dynkin diagrams, where $X$ will turn out to be isomorphic to an affine space with linear action of $T$, in which the maximal-dimensional orbits have codimension 0,1 or 2 .

Remark 15. Observe that the one-parameter subgroup $t \mapsto \lambda(t):=(t, \ldots, t) \in T$ satisfies $\lim _{t \rightarrow \infty} \lambda(t) f=0$ for all $f \in X$. This shows that all irreducible components of $X$ contain 0 ; in particular, $X$ is connected.

4.2. The extremal form. Cohen et al. [2001] proved that on any Lie algebra over a field of characteristic unequal to 2 that is generated by finitely many extremal elements, there is a unique bilinear form $\kappa$ such that $x x y=\kappa(x, y) x$ for all extremal $x$. Moreover, it is shown there that $\kappa$ is symmetric and associative: $\kappa(x, y)=\kappa(y, x)$ and $\kappa(x y, z)=\kappa(x, y z)$ for all $x, y, z$. We call $\kappa$ the extremal form. For the Chevalley algebra $\mathfrak{g}$ of Section 3.2, the extremal form is nonzero on $\mathfrak{g}_{\alpha} \times \mathfrak{g}_{\beta}$ if and only if $\alpha=-\beta$. The form may have a radical contained in the Cartan subalgebra $\mathfrak{h}$.

On the other hand, for any $f \in\left(\mathscr{F}^{*}\right)^{\Pi}$ (not necessarily in $X$ ) the Lie algebra $\mathscr{L}(f)$ is generated by the images of the elements of $\Pi$, which are extremal elements. In particular, for $x \in \Pi$ we have in $\mathscr{L}(f)$

$$
f_{x}(y) x=x x y=\kappa(x, y) x,
$$

where $\kappa$ is the extremal form on $\mathscr{L}(f)$. So if the image of $x$ in $\mathscr{L}(f)$ is nonzero, then $f_{x}(y)=\kappa(x, y)$.

4.3. The Premet relations. Our arguments showing that certain monomials $m:=$ $x_{d} \cdots x_{1}$ are zero in the sandwich algebra $\mathscr{L}(0)$ always depended on the sandwich properties: $x x y=0$ and $x y x z=0$ whenever $x$ is a sandwich element and $y$ and $z$ are arbitrary elements of the Lie algebra. The Premet relations of the following lemma translate such a statement into the following statement: in $\mathscr{L}(f)$ the monomial $\mathrm{m}$ can be expressed in terms of monomials of degree less than $d-1$ and values of $f_{x_{d}}$ on monomials of degree less than $d-1$.

Lemma 16 [Chernousov 1989]. Let $x$ be a nonzero extremal element of a Lie algebra $\mathscr{L}$, and let $f_{x}: \mathscr{L} \rightarrow K$ be the linear function with $x x y=f_{x}(y) x$. Then we have

$$
2 x y x z=f_{x}(y z) x-f_{x}(z) x y-f_{x}(y) x z .
$$

Remark 17. In characteristic 2 the definition of an extremal element $x$ involves the existence of a function $g_{x}$ such that $x y x z=g_{x}(y z) x-g_{x}(z) x y-g_{x}(y) x z$, that is, $g_{x}$ plays the role of $f_{x} / 2$. See [Cohen and Ivanyos 2006, Definition 14]. 
4.4. The parameters. Recall from Section 2 that the restriction map $X \rightarrow\left(V^{*}\right)^{\Pi}$ is injective and has a closed image; a key step in the proof was showing that for $f \in X$ the values $f_{x}(u)$ for $x \in \Pi$ and $u \in F$ depend polynomially on $\left.f\right|_{V}$. In what follows, this will be phrased informally as $f$ can be expressed in $\left.f\right|_{V}$ or $\left.f\right|_{V}$ determines $f$. In this phrase we implicitly assume that $f \in X$, that is, that $\mathscr{L}(f)$ has the maximal possible dimension. In the case of Dynkin diagrams, we will exhibit a small number of values of $f$ in which $f$ can be expressed. For this purpose, the following lemma, which also holds for other graphs, is useful.

Lemma 18. Let $q=x_{d} \cdots x_{1}$ be a monomial of degree $d \geq 2$ and weight $\beta$, and let $z \in \Pi$ be such that $\left\langle\alpha_{z}, \beta\right\rangle \geq-1$. Then $f_{z}(q)$ can be expressed in the parameters $f_{x}(m)$ with monomials $m$ of degree less than $d-1$ and $x \in \Pi$.

Proof. First, if $x_{d}$ is not a $\Gamma$-neighbor of $z$ in $\Gamma$, then

$$
f_{z}\left(x_{d} \cdots x_{1}\right)=\kappa\left(z, x_{d} \cdots x_{1}\right)=-\kappa\left(x_{d} z, x_{d-1} \cdots x_{1}\right)=\kappa\left(0, x_{d-1} \cdots x_{1}\right)=0,
$$

and we are done. So assume that $x_{d}$ is a $\Gamma$-neighbor of $z$. Now

$$
\begin{aligned}
f_{z}\left(x_{d} \cdots x_{1}\right) & =-\kappa\left(x_{d} z, x_{d-1} \cdots x_{1}\right)=\kappa\left(z x_{d}, x_{d-1} \cdots x_{1}\right) \\
& =-f\left(x_{d}, z x_{d-1} \cdots x_{1}\right)=f_{x_{d}}\left(z x_{d-1} \cdots x_{1}\right) .
\end{aligned}
$$

In both cases we have used that the images of $z$ and $x_{d}$ are nonzero in $\mathscr{L}(f)$ for $f \in X$; see Section 4.2. Now $\left\langle\alpha_{z}, \beta-\alpha_{x_{d}}\right\rangle \geq 0$, so Lemma 7 says that $z x_{d-1} \cdots x_{1}$ can be expressed in terms of smaller monomials and values $f_{x}(m)$ for $x \in \Pi$ and monomials $m$ of degree less than $d-1$. Then by linearity of $f_{x_{d}}$, the last expression above can also be expressed in terms of values $f_{x}(m)$ with $x \in \Pi$ and $m$ of degree less than $d-1$.

4.5. Simply laced Dynkin diagrams of finite type. Suppose that $\Gamma$ is a simply laced Dynkin diagram of finite type. Let $\mathfrak{g}$ be the Chevalley algebra with Dynkin diagram $\Gamma$ of Section 3.2. We identify $\mathbb{Z}^{\Pi}$ with the character group of $T=\left(K^{*}\right)^{\Pi}$ in the natural way: we write $\mu$ for the character that sends $t$ to $t^{\mu}=\prod_{x \in \Pi} t_{x}^{\mu_{x}}$. Also let $\Sigma$ be the set of edges of $\Gamma$, and write $\alpha_{e}:=\alpha_{x}+\alpha_{y}$ for $e=\{x, y\} \in \Sigma$.

Theorem 19. Let $\Gamma=(\Pi, \Sigma)$ be a simply laced Dynkin diagram of finite type, obtained from a diagram in Figure 1 by removing vertex 0 . Let $\mathfrak{g}$ be the Chevalley algebra of type $\Gamma$ over the field $K$ of characteristic unequal to 2 . Set $T:=\left(K^{*}\right)^{\Pi}$. Then the variety $X$ is, as a $T$-variety, isomorphic to the vector space $V:=K^{\Sigma}$ on which $T$ acts diagonally with character $-\alpha_{e}$ on the component corresponding to $e \in \Sigma$. For $f$ corresponding to any element in the dense T-orbit $\left(K^{*}\right)^{\Sigma}$, the Lie algebra $\mathscr{L}(f)$ is isomorphic to a fixed Lie algebra.

We first need a lemma that will turn out to describe the generic $\mathscr{L}(f)$. We retain the notation $E_{x}^{0}, H_{x}^{0}, F_{x}^{0} \in \mathfrak{g}$ and $\mathfrak{n}_{+}$from Section 3.2. We denote by $C$ the variety 


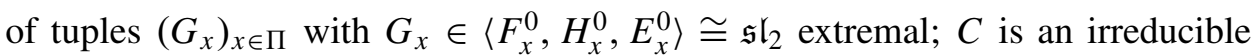
variety.

Lemma 20. For generic $G=\left(G_{x}\right)_{x \in \Pi} \in C$, the Lie subalgebra $\mathfrak{g}^{\prime}$ of $\mathfrak{g}$ generated by the $G_{x}$ has dimension $\operatorname{dim} \mathfrak{n}_{+}$, and moreover $G_{x} G_{x} G_{y}$ is a nonzero multiple of $G_{x}$ for all $x \sim y$.

Proof. By definition $\mathfrak{g}^{\prime}$ is generated by extremal elements; hence it has dimension at most that of $\mathscr{L}(0)$, which is isomorphic to $\mathfrak{n}_{+}$by Theorem 12. The condition that the $G_{x}$ generate a Lie algebra of dimension less than $\operatorname{dim} \mathfrak{n}_{+}$is closed, and the tuple $\left(E_{x}^{0}\right)_{x \in \Pi} \in C$ does not fulfill it. Hence using the irreducibility of $C$, we find that for $G$ in an open dense subset of $C$, the Lie algebra $\mathfrak{g}^{\prime}$ has $\operatorname{dim} \mathfrak{g}^{\prime}=\operatorname{dim} \mathfrak{n}_{+}$. This proves the first statement. The second statement follows directly from the same statement for $\Gamma$ of type $A_{2}$, that is, for $\mathfrak{g}=\mathfrak{s l}_{3}$, where it boils down to the statement that the two copies of $\mathfrak{s l}_{2}$ in $\mathfrak{s l}_{3}$ corresponding to the simple roots are not mutually perpendicular relative to the extremal form in $\mathfrak{s l}_{3}$.

Proof of Theorem 19. By Lemma 18 and Theorem 12, any $f \in X$ is determined by its values $f_{x}(m)$ with $x \in \Pi$ and monomials $m$ of weights $\beta \in \Phi_{+}$such that either $\beta$ has height 1 or $\left\langle\alpha_{x}, \beta\right\rangle \leq-2$. But since $\beta$ is a positive root, the latter inequality cannot hold. Hence $\beta$ has height 1 , so that $m \in \Pi$, and the only $x \in \Pi$ for which $f_{x}(m) \neq 0$ are the neighbors of $m$. Moreover, from the symmetry of the extremal form we conclude that $f_{x}(y)=f_{y}(x)$ for $x, m \in \Pi$ neighbors in $\Gamma$.

We thus find a closed embedding $\Psi: X \rightarrow K^{\Sigma}$ sending $f$ to $\left(f_{x}(y)\right)_{\{x, y\} \in \Sigma}$. Now if we let $T$ act on $K^{\Sigma}$ through the homomorphism

$$
T \rightarrow\left(K^{*}\right)^{\Sigma}, t \mapsto\left(t_{x}^{-1} t_{y}^{-1}\right)_{\{x, y\} \in \Sigma},
$$

then $\Psi$ is $T$-equivariant by the results of Section 4.1. Note that $T$ acts by the character $-\alpha_{e}$ on the component corresponding to $e \in \Sigma$. The fact that $\Gamma$ is a tree readily implies that the characters $\alpha_{e}$ for $e \in \Sigma$ are linearly independent over $\mathbb{Z}$ in the character group of $T$, so that the homomorphism $T \rightarrow\left(K^{*}\right)^{\Sigma}$ is surjective. But then $T$ has finitely many orbits on $K^{\Sigma}$, namely those of the form $\left(K^{*}\right)^{\Sigma^{\prime}} \times\{0\}^{\Sigma \backslash \Sigma^{\prime}}$ with $\Sigma^{\prime} \subseteq \Sigma$.

Now as $\Psi(X)$ is a closed $T$-stable subset of $K^{\Sigma}$, we are done if we can show that $\left(K^{*}\right)^{\Sigma} \cap \Psi(X)$ is nonempty. But this is precisely what Lemma 20 tells us: there exist Lie algebras $\mathfrak{g}^{\prime}$ generated by extremal elements that have the largest possible dimension, and where all coordinates $f_{x}(y)$ with $x \sim y$ are nonzero. This concludes the proof.

Remark 21. The proof above also implies that all Lie algebras described in Lemma 20 are isomorphic. More generally, for any two Lie algebras $\mathfrak{g}^{\prime}$ and $\mathfrak{g}^{\prime \prime}$ with tuples of distinguished, extremal generators $\left(G_{x}^{\prime}\right)_{x \in \Pi}$ and $\left(G_{x}^{\prime \prime}\right)_{x \in \Pi}$ such that 
(i) $G_{x}^{\prime} G_{y}^{\prime}=0$ and $G_{x}^{\prime \prime} G_{y}^{\prime \prime}=0$ for $x \nsim y$,

(ii) $G_{x}^{\prime} G_{x}^{\prime} G_{y}^{\prime}=0$ if and only if $G_{x}^{\prime \prime} G_{x}^{\prime \prime} G_{y}^{\prime \prime}=0$ for $x \sim y$, and

(iii) $\operatorname{dim} \mathfrak{g}^{\prime}=\operatorname{dim} \mathfrak{g}^{\prime \prime}=\operatorname{dim} \mathfrak{n}_{+}$,

there exists an isomorphism $\mathfrak{g}^{\prime} \rightarrow \mathfrak{g}^{\prime \prime}$ mapping each $G_{x}^{\prime}$ to a scalar multiple of $G_{x}^{\prime \prime}$.

4.6. Simply laced Dynkin diagrams of affine type. Suppose now that $\Gamma$ is a simply laced Dynkin diagram of affine type. We retain the notation from Section 3.3. In particular, let $\mathfrak{g}$ be the Chevalley algebra of type $\Gamma^{0}$, the graph induced by $\Gamma$ on $\Pi^{0}=\Pi \backslash\left\{x_{0}\right\}$. To state the analogue of Theorem 19 , we again identify $\mathbb{Z}^{\Pi}$ with the character group of $T=\left(K^{*}\right)^{\Pi}$ and retain the notation $\alpha_{e}$ for $e \in \Sigma$, the edge set of $\Gamma$.

Theorem 22. Let $\Gamma=(\Pi, \Sigma)$ be a simply laced Dynkin diagram of affine type from Figure 1, and let $\Gamma^{0}$ be the finite-type diagram obtained by deleting vertex 0 from $\Gamma$. Let $\mathfrak{g}$ be the Chevalley algebra of type $\Gamma^{0}$ over the field $K$ of characteristic unequal to 2 , and set $T:=\left(K^{*}\right)^{\Pi}$. Then the variety $X$ is, as a $T$-variety, isomorphic to the vector space $V:=K^{\Sigma} \times K$ on which $T$ acts diagonally with character $-\alpha_{e}$ on the component corresponding to $e \in \Sigma$, and with character $-\delta$ on the last component. For all $f \in X$ corresponding to points in some open dense subset of $K^{\Sigma} \times K$, the Lie algebra $\mathscr{L}(f)$ is isomorphic to $\mathfrak{g}$.

Remark 23. Unlike for diagrams of finite type, it is not necessarily true that $T$ has only finitely many orbits on $V$. Indeed, the following three situations occur:

(i) The characters $\alpha_{e}$ (for $e \in \Sigma$ ) and $\delta$ are linearly independent. This is the case for $D_{\text {even }}^{(1)}, E_{7}^{(1)}$, and $E_{8}^{(1)}$. Then $T$ has finitely many orbits on $V$.

(ii) The characters $\alpha_{e}$ for $e \in \Sigma$ are linearly independent, but $\delta$ is in their $\mathbb{Q}$ linear span. This is the case for $A_{\text {even }}^{(1)}, D_{\text {odd }}^{(1)}$ and $E_{6}^{(1)}$. Now the orbits of $T$ in $\left(K^{*}\right)^{\Sigma} \times K^{*}$ have codimension 1 . The character $\delta$, for $A_{\text {even }}^{(1)}$ and $E_{6}^{(1)}$, has full support when expressed in the $\alpha_{e}$; this readily implies that $T$ has finitely many orbits on the complement of $\left(K^{*}\right)^{\Sigma} \times K^{*}$. For $D_{n}^{(1)}$ with $n$ odd, however, $(n-3) / 2$ edge characters get coefficient 0 when $\delta$ is expressed in them, and therefore $T$ still has infinitely many orbits on said complement.

(iii) The characters $\alpha_{e}$ for $e \in \Sigma$ are linearly dependent. This is the case only for $A_{\mathrm{odd}}^{(1)}$, and in fact $\delta$ is then also in the span of the $\alpha_{e}$. Now the $T$-orbits in $\left(K^{*}\right)^{\Sigma} \times K^{*}$ have codimension 2 , and in the complement there are still infinitely many orbits.

This gives some feeling for the parameter space $X$. It would be interesting to determine exactly all isomorphism types of Lie algebras $\mathscr{L}(f)$ with $f \in X$ - but here we confine ourselves to those with $f$ in some open dense subset of $K^{\Sigma} \times K$. 
The proof is very similar to that of Theorem 19. Again, we first prove a lemma that turns out to describe the generic $\mathscr{L}(f)$. Retain the notation $E_{x}^{0}, H_{x}^{0}, F_{x}^{0} \in \mathfrak{g}$ for $x \in \Pi^{0}$. Denote the lowest weight by $\theta \in \Phi_{-}^{0}$, let $E_{x_{0}}^{0}$ and $F_{x_{0}}^{0} \in \mathfrak{g}$ be the elements of the Chevalley basis of weights $\theta$ and $-\theta$, respectively, and set $H_{x_{0}}^{0}:=\left[E_{x_{0}}^{0}, F_{x_{0}}^{0}\right]$. Write $C$ for the irreducible variety of tuples $\left(G_{x}\right)_{x \in \Pi}$ with $G_{x} \in\left\langle F_{x}^{0}, H_{x}^{0}, E_{x}^{0}\right\rangle \cong \mathfrak{s l}_{2}$ extremal.

Lemma 24. For generic $G=\left(G_{x}\right)_{x \in \Pi} \in C$, the $G_{x}$ generate $\mathfrak{g}$, and $G_{x} G_{x} G_{y}$ is a nonzero multiple of $G_{x}$ for all $x \sim y$.

Proof. The first statement is true for $G=\left(E_{x}^{0}\right)_{x \in \Pi}$; this follows from the properties of the Chevalley basis in Section 3.2. Hence by the irreducibility of $C$ it is true generically. The second statement follows, as in Lemma 20, from the same statement in $\mathfrak{s l}_{3}$.

In the following proof we will show that the choice of $\left(G_{x}\right)_{x \in \Pi}$ as in Lemma 24 already gives generic points in $X$, except for the case where $\Gamma$ is of type $A_{\mathrm{odd}}^{(1)}$, for which we give another construction.

Proof of Theorem 22. By Lemma 18 and Theorem 13, any $f \in X$ is determined by its values $f_{x}(m)$ with $x \in \Pi$ and monomials $m$ of weights $\beta \in \Theta$ such that either $\beta$ has height 1 or $\left\langle\alpha_{x}, \beta\right\rangle \leq-2$. In contrast with the case of finite-type diagrams, there do exist pairs $(x, \beta) \in \Pi \times \Theta$ with this latter property, namely, precisely those of the form $\left(x, \delta-\alpha_{x}\right)$. For all $x \in \Pi$, let $m_{x}$ be a monomial that spans the weight space in $\mathscr{L}(0)$ of weight $\delta-\alpha_{x}$; this space is 1-dimensional by Theorem 13. We claim that the $f_{x}\left(m_{x}\right)$ can all be expressed in terms of $f_{x_{0}}\left(m_{x_{0}}\right)$ and values $f_{z}(r)$ with $z \in \Pi$ and $r$ of degree less than $h-2$. Indeed, if $x \neq x_{0}$, then $x_{0}$ occurs exactly once in $m_{x}$; and writing $m_{x}=x_{d} \cdots x_{1} x_{0} y_{e} \cdots y_{1}$ with $x_{1}, \ldots, x_{d}, y_{1}, \ldots, y_{e} \in \Pi^{0}$, we find

$$
\begin{aligned}
f_{x}\left(m_{x}\right) & =\kappa\left(x, x_{d} \cdots x_{1} x_{0} y_{e} \cdots y_{1}\right) \\
& =(-1)^{d+1} \kappa\left(x_{0} x_{1} \cdots x_{d} x, y_{e} \cdots y_{1}\right) \\
& =(-1)^{d} \kappa\left(x_{0},\left[x_{1} \cdots x_{d} x, y_{e} \cdots y_{1}\right]\right) \\
& =(-1)^{d} f_{x_{0}}\left(\left[x_{1} \cdots x_{d} x, y_{e} \cdots y_{1}\right]\right),
\end{aligned}
$$

and the expression $\left[x_{1} \cdots x_{d} x, y_{e} \cdots y_{1}\right]$ can be rewritten in terms of $m_{x_{0}}$ and shorter monomials, using values $f_{z}(r)$ with $r$ of degree less than $d+e=h-2$.

We have now found a closed embedding $X \rightarrow K^{\Sigma} \times K$ which sends $f$ to $\left(\left(f_{x}(y)\right)_{\{x, y\} \in \Sigma}, f_{x_{0}}\left(m_{x_{0}}\right)\right)$; for ease of exposition we will view $X$ as a closed subset of $K^{\Sigma} \times K$. The theorem follows once we can realize generic parameter values in $K^{\Sigma} \times K$ with extremal elements that generate $\mathfrak{g}$. To this end, choose a generic tuple $\left(G_{x}\right)_{x \in \Pi}$ in $C$. By Lemma 24 these generate $\mathfrak{g}$, and they clearly satisfy $G_{x} G_{y}=0$ for $x \not y$. Hence they yield a point in $X$ with $f_{x}(y)=\kappa\left(G_{x}, G_{y}\right) \neq 0$ for $x \sim y$. Furthermore, the parameter $f_{x_{0}}\left(m_{x_{0}}\right)$ equals the extremal form evaluated on $G_{x_{0}}$ 
and the monomial $m_{x_{0}}$ evaluated in the $G_{x}$. Express that monomial in the $G_{x}$ as $\xi E_{x_{0}}^{0}+\eta H_{x_{0}}^{0}+\zeta F_{x_{0}}^{0}$ plus a term perpendicular to $\left\langle E_{x_{0}}^{0}, H_{x_{0}}^{0}, F_{x_{0}}^{0}\right\rangle$, and write $G_{x_{0}}$ as $\xi^{\prime} E_{x_{0}}^{0}+\eta^{\prime} H_{x_{0}}^{0}+\zeta^{\prime} F_{x_{0}}^{0}$. For the degenerate case where $G_{x}=E_{x}^{0}$ for all $x$, we have $\xi, \eta=0$ and $\zeta \neq 0$ (that monomial is a nonzero scalar multiple of the highest root vector, $\left.F_{x_{0}}^{0}\right)$, so that $f_{x_{0}}\left(m_{x_{0}}\right)=\kappa\left(E_{x_{0}}^{0}, \zeta F_{x_{0}}^{0}\right) \neq 0$. Therefore, this parameter is nonzero generically. Hence we have found a point $f \in X \bigcap\left(\left(K^{*}\right)^{\Sigma} \times K^{*}\right)$. Along the lines of Remark 23 we now distinguish three cases:

(i) If the $\alpha_{e}$ (for $e \in \Sigma$ ) and $\delta$ are linearly independent, then $T$ acts transitively on $\left(K^{*}\right)^{\Sigma} \times K^{*}$, and we are done.

(ii) If the $\alpha_{e}$ for $e \in \Sigma$ are linearly independent, but $\delta$ lies in their span, then we now show that we can alter the point $f$ above in a direction transversal to its $T$-orbit. Let $S$ be the torus in the adjoint group of $\mathfrak{g}$ whose Lie algebra is $\mathfrak{h}$, and consider the effect on $f$ of conjugation of $G_{x_{0}}$ with an element $s \in S$, while keeping the other $G_{x}$ fixed. This transforms $G_{x_{0}}=\xi^{\prime} E_{x_{0}}^{0}+\eta^{\prime} H_{x_{0}}^{0}+\zeta^{\prime} F_{x_{0}}^{0}$ in $s^{\theta} \xi^{\prime} E_{x_{0}}^{0}+\eta^{\prime} H_{x_{0}}^{0}+s^{-\theta} \zeta^{\prime} F_{x_{0}}$, and therefore it transforms $f_{x_{0}}\left(m_{x_{0}}\right)$ into

$$
s^{\theta} \xi^{\prime} \zeta \kappa\left(E_{x_{0}}^{0}, F_{x_{0}}^{0}\right)+\eta^{\prime} \eta \kappa\left(H_{x_{0}}^{0}, H_{x_{0}}^{0}\right)+s^{-\theta} \zeta^{\prime} \xi \kappa\left(F_{x_{0}}^{0}, E_{x_{0}}^{0}\right)
$$

while it keeps the parameters $f_{x_{0}}(y)$ with $x_{0} \sim y$ unchanged; these only depend on $\eta^{\prime}$. This shows that we can indeed move $f$ inside $X$ in a direction transverse to its $T$-orbit, and we are done.

(iii) Finally, in the case of $A_{n-1}^{(1)}$ with $n$ even, we first show that tuples in $C$ only give points in a proper closed subset of $K^{\Sigma} \times K$. Here $\mathfrak{g}=\mathfrak{s l}_{n}$ and $\Gamma$ is an $n$ cycle; label its points $0, \ldots, n-1$. Relative to the usual choices of $E_{i}^{0}, H_{i}^{0}, F_{i}^{0}$, the element $G_{i}$ is a matrix with $2 \times 2$-block

$$
\left[\begin{array}{cc}
a_{i} b_{i} & a_{i}^{2} \\
-b_{i}^{2} & -a_{i} b_{i}
\end{array}\right]
$$

on the diagonal in rows (and columns) $i$ and $i+1$ and with zeros elsewhere. We count the rows and columns modulo $n$ so that row 0 is actually row $n$. But then we have $\kappa\left(G_{i}, G_{i+1}\right)=2 a_{i} b_{i} a_{i+1} b_{i+1}$, and this implies

$$
\begin{aligned}
\kappa\left(G_{1}, G_{2}\right) & \kappa\left(G_{3}, G_{4}\right) \cdots \kappa\left(G_{n-1}, G_{0}\right) \\
& =2^{n}\left(a_{1} b_{1}\right)\left(a_{2} b_{2}\right)\left(a_{3} b_{3}\right)\left(a_{4} b_{4}\right) \cdots\left(a_{n-1} b_{n-1}\right)\left(a_{0} b_{0}\right) \\
& =2^{n}\left(a_{0} b_{0}\right)\left(a_{1} b_{1}\right)\left(a_{2} b_{2}\right)\left(a_{3} b_{3}\right) \cdots\left(a_{n-2} b_{n-2}\right)\left(a_{n-1} b_{n-1}\right) \\
& =\kappa\left(G_{0}, G_{1}\right) \kappa\left(G_{2}, G_{3}\right) \cdots \kappa\left(G_{n-2}, G_{n-1}\right) ;
\end{aligned}
$$

so the tuple of parameter values of the tuple $\left(G_{i}\right)_{i=0}^{n-1} \in C$ lies in a proper closed subset $R$ of $K^{\Sigma} \times K$. 
We therefore allow the tuple $\left(G_{i}\right)_{i=0}^{n-1}$ to vary in a slightly larger variety $C^{\prime} \supset C$ as follows: the conditions on $G_{1}, \ldots, G_{n-1}$ remain the same, but $G_{0}$ is now allowed to take the shape

$$
\left[\begin{array}{ccccc}
-a_{0} b_{0} & 0 & \ldots & 0 & -b_{0}^{2} \\
c_{2} a_{0} & 0 & \ldots & 0 & c_{2} b_{0} \\
\vdots & \vdots & & \vdots & \vdots \\
c_{n-1} a_{0} & 0 & \ldots & 0 & c_{n-1} b_{0} \\
a_{0}^{2} & 0 & \ldots & 0 & a_{0} b_{0}
\end{array}\right]
$$

(which is extremal since it has rank 1 and trace 0 ), subject to the equations

$$
b_{i} c_{i}+a_{i} c_{i+1}=0 \quad \text { for } i=2, \ldots, n-2,
$$

which ensure that $G_{0}$ commutes with $G_{2}, \ldots, G_{n-2}$. Still, any tuple in an open neighborhood $U \subseteq C^{\prime}$ of our original tuple $\left(G_{i}\right)_{i=0}^{n-1}$ (with generic $a_{i}$ and $b_{i}$ but all $c_{i}$ equal to 0$)$ generates $\mathfrak{s l}_{n}$. We now argue that the differential $d$ at $\left(G_{i}\right)_{i=0}^{n-1}$ of the map $U \rightarrow X \subseteq K^{\Sigma} \times K$ sending a tuple to the parameters that it realizes has rank $|\Sigma|+1$, as required. Indeed, the $T$-action already gives a subspace of dimension $|\Sigma|-1$, tangent to $R$. Making $c_{2}$ (and hence all $c_{i}$ ) nonzero adds $-2 a_{1}^{2} c_{2} a_{0}$ to $\kappa\left(G_{0}, G_{1}\right)$ and $2 b_{n-1}^{2} c_{n-1} b_{0}$ to $\kappa\left(G_{0}, G_{n-1}\right)$, and it fixes all other $\kappa\left(G_{i}, G_{j}\right)$. We show that this infinitesimal direction is not tangent to $R$ : it adds

$$
2^{n} b_{n-1}^{2} c_{n-1} b_{0}\left(a_{1} b_{1}\right)\left(a_{2} b_{2}\right) \cdots\left(a_{n-3} b_{n-3}\right)\left(a_{n-2} b_{n-2}\right)
$$

to the left side of (2), and

$$
-2^{n} a_{1}^{2} c_{2} a_{0}\left(a_{2} b_{2}\right)\left(a_{3} b_{3}\right) \cdots\left(a_{n-2} b_{n-2}\right)\left(a_{n-1} b_{n-1}\right)
$$

to the right side. Dividing these expressions by common factors, the first becomes $2^{n} b_{n-1} c_{n-1} b_{0} b_{1}$ and the second $-2^{n} a_{1} c_{2} a_{0} a_{n-1}$. These expressions are not equal generically, even modulo the equations (3) relating the $c_{i}$ to the $a_{i}$ and $b_{i}$; indeed, these equations do not involve $a_{0}, a_{1}, a_{n-1}, b_{0}, b_{1}, b_{n-1}$.

Note that varying $c_{2}$ may also effect the parameter $f_{x_{0}}\left(m_{x_{0}}\right)$, but in any case the above shows that the composition of the differential $d$ with projection onto $K^{\Sigma}$ is surjective. On the other hand, conjugation with the torus $S$ as in case (ii) yields a vector in the image of $d$ which is supported only on the factor $K$ corresponding to $\delta$. This concludes the proof that $d$ has full rank.

\section{Notes}

5.1. Recognizing the simple Lie algebras. Going through the proof that $X$ is an affine variety, one observes that the map $\left.f \mapsto f\right|_{V}$ is not only injective on $X$, but 
even on

$$
X^{\prime}(\Gamma):=\left\{f \in\left(\mathscr{F}^{*}\right)^{\Pi} \mid \text { for all } x \in \Pi: x \neq 0 \text { in } \mathscr{L}(f)\right\} \supseteq X .
$$

The same is true for the map $f \mapsto\left(\left(f_{x}(y)\right)_{\{x, y\} \in \Sigma}\right)$ in the case where $\Gamma$ is a Dynkin diagram of finite type, and for the map $f \mapsto\left(\left(f_{x}(y)\right)_{\{x, y\} \in \Sigma}, f_{x_{0}}\left(m_{x_{0}}\right)\right)$ in the case where $\Gamma$ is a Dynkin diagram of affine type. This shows that, for these Dynkin diagrams, $X^{\prime}(\Gamma)$ is actually equal to $X$, whence the following theorem.

Theorem 25. Suppose that $\Gamma$ is a Dynkin diagram of finite or affine type. Let $\mathscr{L}$ be any Lie algebra, over a field of characteristic unequal to 2 , which is generated by nonzero extremal elements $G_{x}$ for $x \in \Pi$ in which the commutation relations $G_{x} G_{y}=0$ hold for $x \ngtr y$. Define $f \in\left(\mathscr{F}^{*}\right)^{\Pi}$ by the condition that $G_{x} G_{x} u=$ $f_{x}(u) G_{x}$ holds in $\mathscr{L}$. Then $f \in X$ and $\mathscr{L}$ is a quotient of $\mathscr{L}(f)$.

This theorem could well prove useful for recognizing the Chevalley algebras $\mathfrak{g}$ : if $f$ corresponds to a point in the open dense subset of $K^{\Sigma} \times K$ referred to in Theorem 22 , then one concludes that $\mathscr{L}$ is a quotient of $\mathfrak{g}$. Hence if $\mathfrak{g}$ is a simple Lie algebra, then $\mathscr{L}$ is isomorphic to $\mathfrak{g}$. It is not clear to us whether, for general $\Gamma$, the image of $X^{\prime}(\Gamma)$ in $\left(V^{*}\right)^{\Pi}$ is closed; this is why we chose to work with $X$ instead.

5.2. Other graphs. Our methods work very well for Dynkin diagrams, but for more general graphs new ideas are needed to determine $\mathscr{L}(0), X$, and $\mathscr{L}(f)$ for $f \in X$. The relation with the Kac-Moody algebra of $\Gamma$ may be much tighter than we proved in Section 3.1. General questions of interest are: Is $X$ always an affine space? Is there always a generic Lie algebra? We expect the answers to both questions to be negative, but do not have any counterexamples.

The references [in 't panhuis et al. 2007; Postma 2007; Roozemond 2005] contain other series of graphs which exhibit the same properties as we have proved here: the variety $X$ is an affine space, and generic points in it correspond to simple Lie algebras of types $A_{n}, C_{n}, B_{n}, D_{n}$. In fact, the graph that they find for $C_{n}$ is just the finite-type Dynkin diagram of type $A_{2 n}$. This also follows easily from our results: take $2 n$ generic extremal elements $\left(G_{x}\right)_{x}$ in $\mathfrak{s l}_{2 n+1}$ as in Lemma 20. These generate a subalgebra of $\mathfrak{s l}_{2 n+1}$ of dimension $\left(\begin{array}{c}2 n+1 \\ 2\end{array}\right)$ by that same lemma, and if we consider them as matrices, their images span a subspace $W$ of dimension $2 n$ in $K^{2 n+1}$. It is not hard to write down an explicit, nondegenerate skew symmetric form on $W$ with respect to which the $G_{x}$ are skew - hence the Lie algebra generated by them is $\mathfrak{s p}_{2 n}$.

\section{References}

[Billig 1990] Y. V. Billig, "Modular affine Lie algebras", Mat. Sb. 181:8 (1990), 1130-1143. In Russian; translated in Math. USSR-Sb. 70:2 (1991), 573-586. MR 91k:17016 Zbl 0715.17024 
[Carter 1972] R. W. Carter, Simple groups of Lie type, Pure and Applied Mathematics 28, Wiley, London, 1972. MR 53 \#10946 Zbl 0248.20015

[Chernousov 1989] V. I. Chernousov, "The Hasse principle for groups of type E8", Dokl. Akad. Nauk SSSR 306:5 (1989), 1059-1063. In Russian; translated in Soviet Math. Dokl. 39:3 (1989), 592-596. MR 90m:11055 Zbl 0703.20040

[Cohen and Ivanyos 2006] A. M. Cohen and G. Ivanyos, "Root filtration spaces from Lie algebras and abstract root groups", J. Algebra 300:2 (2006), 433-454. MR 2008a:17008 Zbl 1109.51006

[Cohen et al. 2001] A. M. Cohen, A. Steinbach, R. Ushirobira, and D. Wales, "Lie algebras generated by extremal elements", J. Algebra 236:1 (2001), 122-154. MR 2001k:17007 Zbl 1076.17500

[Kac 1990] V. G. Kac, Infinite-dimensional Lie algebras, 3rd ed., Cambridge University Press, 1990. MR 92k:17038 Zbl 0716.17022

[Kostrikin 1981] A. I. Kostrikin, "Sandwiches in Lie algebras", Math. USSR, Sb. 38 (1981), 1-9. Zbl 0449.17003

[in 't panhuis et al. 2007] J. C. H. W. in 't panhuis, E. J. Postma, and D. A. Roozemond, "Extremal presentations for classical Lie algebras", Preprint, 2007. arXiv 0705.2332v3

[Postma 2007] E. J. Postma, From Lie algebras to geometry and back, Thesis, Technische Universiteit Eindhoven, 2007, Available at http://alexandria.tue.nl/extra2/200710440.pdf.

[Premet and Strade 1997] A. Premet and H. Strade, "Simple Lie algebras of small characteristic, I: Sandwich elements”, J. Algebra 189:2 (1997), 419-480. MR 98e:17029 Zbl 0878.17019

[Roozemond 2005] D. A. Roozemond, Lie algebras generated by extremal elements, Master's thesis, Technische Universiteit Eindhoven, 2005, Available at http://alexandria.tue.nl/extra1/afstversl/ wsk-i/roozemond2005.pdf.

[Seligman 1967] G. B. Seligman, Modular Lie algebras, Ergebnisse der Mathematik und ihrer Grenzgebiete 40, Springer, New York, 1967. MR 39 \#6933 Zbl 0189.03201

[Strade 2004] H. Strade, Simple Lie algebras over fields of positive characteristic, I: Structure theory, de Gruyter Expositions in Mathematics 38, de Gruyter, Berlin, 2004. MR 2005c:17025 Zbl 1074.17005

[Zelmanov and Kostrikin 1990] E. I. Zel'manov and A. I. Kostrikin, "A theorem on sandwich algebras”, Trudy Mat. Inst. Steklov. 183 (1990), 106-111, 225. In Russian; translated in Proc. Steklov Inst. Math. 183:4 (1991), 121-126. MR 92h:17007 Zbl 0729.17006

Communicated by Efim Zelmanov

Received 2007-08-17 Revised 2008-03-06 Accepted 2008-05-27

j.draisma@tue.nl

Department of Mathematics and Computer Science, Technische Universiteit Eindhoven, P.O. Box 513, 5600 MB Eindhoven, The Netherlands http://www.win.tue.nl/ jdraisma/

j.c.h.w.panhuis@tue.nl

Department of Mathematics and Computer Science, Technische Universiteit Eindhoven, P.O. Box 513, 5600 MB Eindhoven, The Netherlands http://www.win.tue.nl/ jpanhuis/ 


\title{
Integral traces of singular values of weak Maass forms
}

\author{
William Duke and Paul Jenkins
}

\begin{abstract}
We define traces associated to a weakly holomorphic modular form $f$ of arbitrary negative even integral weight and show that these traces appear as coefficients of certain weakly holomorphic forms of half-integral weight. If the coefficients of $f$ are integral, then these traces are integral as well. We obtain a negative weight analogue of the classical Shintani lift and give an application to a generalization of the Shimura lift.
\end{abstract}

\section{Introduction}

Recently there has been a resurgence of interest in the classical theory of singular moduli, these being the values of the modular $j$-function at quadratic irrationalities. This resurgence is due largely to the influential papers of Borcherds [1995; 1998] and Zagier [2002]. The present paper arose from a suggestion, made at the end of Zagier's paper, to extend some of the results given there on traces of singular moduli to higher weights. One such generalization has been given recently by Bringmann and Ono [2007], who provide an identity for the traces associated to certain Maass forms in terms of the Fourier coefficients of half-integral weight Poincaré series. However, it does not seem to be known when these traces are integral or even rational. Here we will identify the traces associated to a weakly holomorphic form $f$ of negative-integral weight with the coefficients of certain weakly holomorphic forms of half-integral weight and show that these coefficients are integral when the coefficients of $f$ are integral. We will use this identification to obtain a negative weight analogue of the classical Shintani lift. We also give an application to Borcherds's generalization of the Shimura lift to weakly holomorphic modular forms.

MSC2000: primary 11F30; secondary 11F37.

Keywords: weak Maass forms, weakly holomorphic modular forms, traces of singular moduli. Duke is supported by NSF grant DMS-0355564, and Jenkins is supported by NSF grant DMS0603271. 
Recall that a weakly holomorphic modular form of weight $k$, where $k \in 2 \mathbb{Z}$, is a holomorphic function $f$ on the upper half-plane $\mathcal{H}$ that satisfies

$$
\left(\left.f\right|_{k} \gamma\right)(\tau)=(c \tau+d)^{-k} f\left(\frac{a \tau+b}{c \tau+d}\right)=f(\tau) \quad \text { for all } \gamma=\left(\begin{array}{ll}
a & b \\
c & d
\end{array}\right) \in \Gamma=\operatorname{PSL}(2, \mathbb{Z})
$$

and that has a $q$-expansion $f(\tau)=\sum_{n} a(n) q^{n}$ with $a(n)=0$ for all but finitely many $n<0$; here, as usual, $q=e(\tau)=e^{2 \pi i \tau}$. Let $M_{k}^{!}$denote the vector space of all weakly holomorphic modular forms of weight $k$. Similarly, for $k=s+1 / 2$ with $s \in$ $\mathbb{Z}$, let $M_{k}^{!}$denote the space of holomorphic functions on $\mathcal{H}$ that transform like $\theta^{2 k}$ under $\Gamma_{0}(4)$, have at most poles in the cusps, and have a $q$-expansion supported on integers $n$ with $(-1)^{s} n \equiv 0,1(\bmod 4)$. Here, as usual, $\theta(\tau)=\sum_{n \in \mathbb{Z}} q^{n^{2}}$. For any $k$, let $M_{k} \subset M_{k}^{!}$denote the subspace of holomorphic forms and $S_{k} \subset M_{k}$ the subspace of cusp forms.

In this paper $d$ is always an integer with $d \equiv 0,1(\bmod 4)$, and $D$ is always a fundamental discriminant (possibly 1 ). Suppose $d D<0$ and $F$ is a $\Gamma$-invariant function on $\mathcal{H}$. Define the twisted trace

$$
\operatorname{Tr}_{d, D}(F)=\sum_{Q} w_{Q}^{-1} \chi(Q) F\left(\tau_{Q}\right)
$$

where the sum is over a complete set of $\Gamma$-inequivalent positive definite integral quadratic forms $Q(x, y)=a x^{2}+b x y+c y^{2}$ with discriminant $d D=b^{2}-4 a c$, and

$$
\tau_{Q}=\frac{-b+\sqrt{d D}}{2 a} \in \mathcal{H}
$$

is the associated CM point. Here $w_{Q}$ is equal to 1 unless $Q \sim a\left(x^{2}+y^{2}\right)$ or $Q \sim a\left(x^{2}+x y+y^{2}\right)$, in which case $w_{Q}$ is equal to 2 or 3 , respectively. Also

$$
\chi(Q)=\chi(a, b, c)= \begin{cases}\chi_{D}(r) & \text { if }(a, b, c, D)=1 \text { and } \\ & Q \text { represents } r, \text { where }(r, D)=1 ; \\ 0 & \text { if }(a, b, c, D)>1,\end{cases}
$$

where $\chi_{D}$ is the Kronecker symbol. It is known that $\chi$ is well defined on classes, that $\chi$ restricts to a real character (a genus character) on the group of primitive classes, and that all such characters arise this way.

For the usual $j$-function $j=E_{4}^{3} / \Delta \in M_{0}^{!}$with Fourier expansion

$$
j(\tau)=q^{-1}+744+196884 q+21493760 q^{2}+\cdots,
$$

it is classical that the value $j\left(\tau_{Q}\right)$ is an algebraic integer in an abelian extension of $\mathbb{Q}(\sqrt{d D})$. Let $j_{1}=j-744$. Zagier [2002] showed that for a fundamental 
discriminant $D \neq 1$ we have

$$
\begin{gathered}
q^{-|D|}+\sum_{d>0} d^{-1 / 2} \operatorname{Tr}_{d, D}\left(j_{1}\right) q^{|d|} \in M_{1 / 2}^{!} \quad \text { if } D<0, \\
q^{-|D|}-D^{-1 / 2} \sum_{d<0} \operatorname{Tr}_{d, D}\left(j_{1}\right) q^{|d|} \in M_{3 / 2}^{!} \quad \text { if } D>0,
\end{gathered}
$$

and that both forms have integral Fourier coefficients. For instance, when $D=-3$ and $D=5$ we have the two weakly holomorphic forms

$$
\begin{array}{r}
q^{-3}-248 q+26752 q^{4}-85995 q^{5}+\cdots \in M_{1 / 2}^{!} \text {and } \\
q^{-5}+85995 q^{3}-565760 q^{4}+52756480 q^{7}+\cdots \in M_{3 / 2}^{!},
\end{array}
$$

and $\operatorname{Tr}_{5,-3}\left(j_{1}\right)=\operatorname{Tr}_{-3,5}\left(j_{1}\right)=j\left(\frac{1+\sqrt{-15}}{2}\right)-j\left(\frac{1+\sqrt{-15}}{4}\right)=-85995 \sqrt{5}$.

In this paper we will give such a result when $j_{1}$ is replaced by a function $f$ of negative weight. To state it, first define the Maass raising operator $\partial_{k}$ in $\tau=x+i y$ :

$$
\partial_{k}=\mathcal{D}-\frac{k}{4 \pi y}, \quad \text { where } \mathcal{D}=\frac{1}{2 \pi i} \frac{d}{d \tau}=q \frac{d}{d q} .
$$

Now $\partial_{k}\left(\left.f\right|_{k} \gamma\right)=\left.\left(\partial_{k} f\right)\right|_{k+2} \gamma$ for any $\gamma \in \operatorname{PSL}(2, \mathbb{R})$. Thus, if $f \in M_{2-2 s}^{!}$for $s \in \mathbb{Z}^{+}$, the function $\partial^{s-1} f$ is $\Gamma$-invariant, where

$$
\partial^{s-1} \equiv(-1)^{s-1} \partial_{-2} \circ \partial_{-4} \circ \cdots \circ \partial_{4-2 s} \circ \partial_{2-2 s} .
$$

After Maass we know that $\partial^{s-1} f$ is an eigenfunction of the Laplacian

$$
\Delta=-y^{-2}\left(\frac{\partial^{2}}{\partial x^{2}}+\frac{\partial^{2}}{\partial y^{2}}\right)
$$

with eigenvalue $s(1-s)$, so $\partial^{s-1} f$ is a weak Maass form (see for example [Bruinier et al. 2008, page 162] for a precise definition). Using a method that readily generalizes, Zagier [2002] showed in special cases that $\partial^{s-1} f$ is a rational function of $j$ and $h=E_{2}^{*} E_{4} E_{6} / \Delta$, where

$$
E_{2}^{*}(\tau)=1-24 \sum_{n \geq 1} \sigma(n) q^{n}-\frac{3}{\pi y}
$$

is the nonholomorphic weight 2 Eisenstein series and $\sigma(n)=\sum_{m \mid n} m$. For a CM point like $\tau_{Q}$ given in (1), it was shown by Ramanujan [1914, Equation (23), page 33 ] that $h\left(\tau_{Q}\right)$ is algebraic. More precisely, $h\left(\tau_{Q}\right) \in \mathbb{Q}\left(j\left(\tau_{Q}\right)\right)$; see [Masser 1975, Theorem A1, page 114]. Using this, we can deduce the remarkable fact that for any $f \in M_{2-2 s}^{!}$with $s \geq 1$ and with rational Fourier coefficients, the "singular" value of the weak Maass form $\partial^{s-1} f\left(\tau_{Q}\right)$ is algebraic. We are thus motivated to study $\operatorname{Tr}_{d, D}\left(\partial^{s-1} f\right)$ for such $f$. 
For $D$ a fundamental discriminant, let $\hat{s}=s$ if $(-1)^{s} D>0$ and $\hat{s}=1-s$ otherwise. It is also convenient to set

$$
\operatorname{Tr}_{d, D}^{*}(f)=(-1)^{\lfloor(\hat{s}-1) / 2\rfloor}|d|^{-\hat{s} / 2}|D|^{(\hat{s}-1) / 2} \operatorname{Tr}_{d, D}\left(\partial^{s-1} f\right) .
$$

Suppose $f \in M_{2-2 s}^{!}$for $s \geq 2$ has Fourier coefficients $a(n)$. For $D$ fundamental, define the $D$-th Zagier lift of $f$ to be

$$
\begin{aligned}
\mathfrak{Z}_{D} f(\tau)=\sum_{m>0} a(-m) m^{s-\hat{s}} \sum_{n \mid m} \chi_{D}(n) n^{\hat{s}-1} q^{-m^{2}|D| / n^{2}} & \\
& +\frac{1}{2} L\left(1-s, \chi_{D}\right) a(0)+\sum_{d: d D<0} \operatorname{Tr}_{d, D}^{*}(f) q^{|d|} .
\end{aligned}
$$

The linear map $f \mapsto \mathfrak{Z}_{D}(f)$ is a negative weight analogue of the Shintani lift on integral weight cusp forms. This follows from our main result, whose proof will be completed in Section 5 .

Theorem 1. Suppose $f \in M_{2-2 s}^{!}$for an integer $s \geq 2$. If $D$ is a fundamental discriminant with $(-1)^{s} D>0$, we have that $\mathfrak{Z}_{D} f \in M_{3 / 2-s}^{!}$, while if $(-1)^{s} D<0$, then $\mathfrak{Z}_{D} f \in M_{s+1 / 2}^{!}$. If $f$ has integral Fourier coefficients, then so does $\mathfrak{Z}_{D} f$.

Here we will not treat the case $s=1$, which requires special considerations and which can be dealt with by the methods of [Zagier 2002]. Furthermore, when $s=2,3,4,5,7$, Theorem 1 can also be deduced from results of [Zagier 2002]. The first new example occurs when $s=6$ and $D=1$, where we have the pair

$$
\begin{aligned}
f(\tau) & =E_{14}(\tau) / \Delta(\tau)^{2}=q^{-2}+24 q^{-1}-196560-47709536 q+\cdots \in M_{-10}^{!}, \\
\mathfrak{Z}_{1} f(\tau) & =q^{-4}+56 q^{-1}+390+15360 q^{3}+42264 q^{4}+615240 q^{7}+\cdots \in M_{-9 / 2}^{!} .
\end{aligned}
$$

Here $-\frac{1}{2} \zeta(-5) \cdot 196560=390$ and the first few values of $\operatorname{Tr}_{d, 1}^{*}(f)$ are

$$
3^{-4} \partial^{5} f\left(\frac{1+\sqrt{-3}}{2}\right)=15360, \quad 2^{-7} \partial^{5} f(i)=42264, \quad 7^{-3} \partial^{5} f\left(\frac{1+\sqrt{-7}}{2}\right)=615240 .
$$

Similarly, when $D=-3$ we have

$$
\mathfrak{Z}_{-3} f(\tau)=2^{11} q^{-12}-8 q^{-3}-15360 q-53319598080 q^{4}+\cdots \in M_{13 / 2}^{!} .
$$

The main new difficulty in proving Theorem 1 comes from the existence of cusp forms in $M_{2 s}^{!}$. The method of Poincaré series adapts nicely to handle it. A key dividend of the method is the last statement of Theorem 1, showing that the integrality of coefficients is preserved under the lift.

Remarks. First, it follows from Theorem 1 that if $(-1)^{s} D>0$, then the image $\mathfrak{Z}_{D}(f) \in M_{3 / 2-s}^{!}$is determined by its principal part and hence by the principal part of $f$. Furthermore, $a(0)$ is divisible by the denominator of each of the $L$-values $\frac{1}{2} L\left(1-s, \chi_{D}\right)$, provided that the Fourier coefficients of $f$ are integral. Using wellknown properties of the generalized Bernoulli numbers, one can reproduce the 
divisibility properties that follow from work of Siegel [1969, pages 254-256]. On the other hand, if $(-1)^{s} D<0$ then $\frac{1}{2} L\left(1-s, \chi_{D}\right)=0$.

Next, it can be shown that the Zagier lift is compatible with the Hecke operators. For details, see the end of Section 5.

Finally, using a theta lift, Bruinier and Funke [2006] have generalized Zagier's result in various other ways, for instance to higher levels, where the existence of cusp forms in the dual weight is also a complication; see also [Funke 2007].

As another application of these methods, we will give a simple proof of a basic property of the Shimura lift for weakly holomorphic modular forms. For

$$
g(\tau)=\sum_{n} b(n) q^{n} \in M_{s+1 / 2}^{!}
$$

with $s \in \mathbb{Z}^{+}$and $D$ fundamental with $(-1)^{s} D>0$, define the $D$-th Shimura lift of $g$ by

$$
\mathscr{Y}_{D} g(\tau)=\frac{1}{2} L\left(1-s, \chi_{D}\right) b(0)+\sum_{m>0}\left(\sum_{n \mid m} \chi_{D}(n) n^{s-1} b\left(m^{2}|D| / n^{2}\right)\right) q^{m} .
$$

When $g$ is holomorphic, this is the usual definition. We will repeatedly use the basic fact that $\mathscr{Y}_{D} g \in M_{2 s}$ if $g \in M_{s+1 / 2}$; see [Kohnen and Zagier 1981]. Recall that a $\mathrm{CM}$ point is a point in $\mathcal{H}$ of the form $\left(-b+\sqrt{b^{2}-4 a c}\right) / 2 a$ for integral $a, b, c$. The proof of the following result will be completed in Section 6. In the case $D=1$, it is due to Borcherds [1998] and follows from a special case of [Theorem 14.3] there; see [Example 14.4].

Theorem 2. For $g \in M_{s+1 / 2}^{!}$with $s \geq 2$ and $D$ a fundamental discriminant with $(-1)^{s} D>0$, the lift $\mathscr{S}_{D} g$ is a meromorphic modular form of weight $2 s$ for $\Gamma$ whose possible poles are of order at most $s$ and occur at CM points.

\section{Weakly holomorphic forms}

In this section we will define a canonical basis for the space $M_{k}^{!}$for any $k=s+1 / 2$ with $s \in \mathbb{Z}$ in which all basis elements have integral Fourier coefficients. Then we will construct forms in $M_{k}^{!}$when $s \geq 2$ using Poincaré series.

We begin by recalling the canonical basis for $M_{2 s}^{!}$defined in [Duke and Jenkins 2008] for any $s \in \mathbb{Z}$. Write $2 s=12 \ell+k^{\prime}$ with uniquely determined $\ell \in \mathbb{Z}$ and $k^{\prime} \in\{0,4,6,8,10,14\}$, so that if $\ell \geq 0$, then $\ell$ is the dimension of the space $S_{2 s}$ of cusp forms of weight $2 s$. For every integer $m \geq-\ell$, there exists a unique $f_{2 s, m} \in M_{2 s}^{!}$with a $q$-expansion of the form $f_{2 s, m}(\tau)=q^{-m}+\sum_{n>\ell} a_{2 s}(m, n) q^{n}$, and together they form a basis for $M_{2 s}^{!}$. The basis element $f_{2 s, m}$ can be given explicitly in the form $f_{2 s, m}=f_{2 s} P(j)$, where $f_{2 s}=f_{2 s,-\ell}=\Delta^{\ell} E_{k^{\prime}}$ and $P$ is a polynomial of degree $m+\ell$. As shown in [Duke and Jenkins 2008], the basis 
elements have the generating function

$$
\sum_{m \geq-\ell} f_{2 s, m}(z) q^{m}=\frac{f_{2 s}(z) f_{2-2 s}(\tau)}{j(\tau)-j(z)}=-\sum_{m \geq \ell+1} f_{2-2 s, m}(\tau) r^{m},
$$

where $r=e(z)$. It follows from this that the coefficients $a_{2 s}(m, n)$ are integral and satisfy the duality relation

$$
a_{2 s}(m, n)=-a_{2-2 s}(n, m) .
$$

In order to formulate a similar result for $M_{k}^{!}$when $k=s+1 / 2$ with $s \in \mathbb{Z}$, let $\ell$ be defined by $2 s=12 \ell+k^{\prime}$ as above. By the Shimura correspondence given in [Kohnen 1980], one finds that the maximal order of a nonzero $f \in M_{k}^{!}$at $i \infty$ is

$$
A= \begin{cases}2 \ell-(-1)^{s} & \text { if } \ell \text { is odd }, \\ 2 \ell & \text { otherwise. }\end{cases}
$$

If $B<A$ is the next admissible exponent we can construct functions in $M_{k}^{!}$of the form

$$
f_{k}(\tau)=q^{A}+O\left(q^{B+4}\right) \quad \text { and } \quad f_{k}^{*}(\tau)=q^{B}+O\left(q^{B+4}\right) .
$$

If we write $s=12 a+b$, where $b \in\{6,8,9,10,11,12,13,14,15,16,17,19\}$, then $f_{k}$ and $f_{k}^{*}$ can be given explicitly in the form

$$
f_{k}(\tau)=\Delta(4 \tau)^{a} f_{b+1 / 2}(\tau) \text { and } f_{k}^{*}(\tau)=\Delta(4 \tau)^{a} f_{b+1 / 2}^{*}(\tau),
$$

where the forms $f_{b+1 / 2}, f_{b+1 / 2}^{*} \in M_{b+1 / 2}$ are given in the appendix and have integral Fourier coefficients. Using them, it is easy to construct a unique basis for $M_{k}^{!}$ consisting of functions of the form

$$
f_{k, m}(\tau)=q^{-m}+\sum_{n>A} a_{k}(m, n) q^{n},
$$

where $m \geq-A$ has $(-1)^{s-1} m \equiv 0,1(\bmod 4)$. Here $f_{k,-A}=f_{k}$ and $f_{k,-B}=f_{k}^{*}$. This can be done recursively: $f_{k, m}(\tau)$ is obtained by multiplying $f_{k, m-4}(\tau)$ by $j(4 \tau)$ and then subtracting a suitable linear combination of the forms $f_{k, m^{\prime}}(\tau)$ with $m^{\prime}<m$. We also have the following generating function, whose proof is similar to Zagier's proof [2002] of the $k=1 / 2$ case:

$$
\sum_{m} f_{k, m}(z) q^{m}=\frac{f_{k}(z) f_{2-k}^{*}(\tau)+f_{k}^{*}(z) f_{2-k}(\tau)}{j(4 \tau)-j(4 z)}=-\sum_{m} f_{2-k, m}(\tau) r^{m} .
$$

This and the fact that $f_{k}$ and $f_{k}^{*}$ have integral Fourier coefficients gives the following result.

Proposition 1. The Fourier coefficients $a_{k}(m, n)$ defined in (8) are integral and satisfy the duality relation

$$
a_{k}(m, n)=-a_{2-k}(n, m) \quad \text { for all } m, n \in \mathbb{Z} .
$$


Another way to construct weakly holomorphic forms is by Poincaré series. Here we will only need them for $k=s+1 / 2$, where $s \geq 2$. Set $j(\gamma, \tau)=\theta(\gamma \tau) / \theta(\tau)$ for $\gamma \in \Gamma_{0}(4)$. For $m \in \mathbb{Z}$, define the Poincaré series

$$
P_{k, m}(\tau)=\sum_{\gamma \in \Gamma_{\infty} \backslash \Gamma_{0}(4)} e(m \gamma \tau) j(\gamma, \tau)^{-2 k}
$$

where $\Gamma_{\infty}$ is the subgroup of translations in $\Gamma_{0}(4)$. For $k \geq 5 / 2$, this series is absolutely convergent and represents a weakly holomorphic form of weight $k$ for $\Gamma_{0}(4)$, but it is not in $M_{k}^{!}$since its Fourier coefficients are not supported on $n$ with $(-1)^{s} n \equiv 0,1(\bmod 4)$. When $m=0$, the Poincaré series is an Eisenstein series that Cohen [1975] projected to a form in $M_{k}$ and whose Fourier coefficients are expressed in terms of the values of Dirichlet $L$-functions at $1-s$. When $m>0$, Kohnen [1985] showed how to obtain in this way cusp forms in $S_{k}$. Bruinier et al. [2006] observed that a similar procedure works for $m<0$. Petersson [1950] had explicitly computed the Fourier expansions of $P_{k, m}$ in terms of Bessel functions and Kloosterman sums, and the projections $g_{k, m}$ of $P_{k, m}$ to $M_{k}^{!}$have Fourier expansions that are simple modifications of these. To give them, for $m, n \in \mathbb{Z}$ and $c \in \mathbb{Z}^{+}$with $c \equiv 0(\bmod 4)$, let

$$
K_{k}(m, n ; c)=\sum_{a(\bmod c)}\left(\frac{c}{a}\right) \varepsilon_{a}^{2 k} e\left(\frac{m a+n \bar{a}}{c}\right)
$$

be the Kloosterman sum, where $\left(\frac{c}{a}\right)$ is the extended Legendre symbol and

$$
\varepsilon_{a}=\left\{\begin{array}{lll}
1 & \text { if } a \equiv 1 \quad(\bmod 4), \\
i & \text { if } a \equiv 3 \quad(\bmod 4)
\end{array}\right.
$$

Also, let $\delta_{\text {odd }}(n)=1$ if $n$ is odd and $\delta_{\text {odd }}(n)=0$ otherwise.

Proposition 2. Suppose $k=s+1 / 2$, where $s \geq 2$. Then, for any nonzero integer $m$ with $(-1)^{s} m \equiv 0,1(\bmod 4)$, there exists a form $g_{k, m} \in M_{k}^{!}$with Fourier expansion

$$
g_{k, m}(\tau)=q^{m}+\sum_{\substack{n \geq 1 \\(-1)^{s} n \equiv 0,1(\bmod 4)}} b_{k}(m, n) q^{n}
$$

where for $(-1)^{s} \equiv 0,1(\bmod 4)$ the coefficient $b_{k}(m, n)$ is given explicitly by the absolutely convergent sum

$$
\begin{aligned}
b_{k}(m, n)=2 \pi i^{-k}\left|\frac{n}{m}\right|^{k-1 / 2} & \sum_{\substack{c>0 \\
c \equiv 0(\bmod 4)}}\left(1+\delta_{\text {odd }}(c / 4)\right) c^{-1} K_{k}(m, n ; c) \\
& \times \begin{cases}I_{k-1}(4 \pi \sqrt{|m n|} / c) & \text { if } m<0, \\
J_{k-1}(4 \pi \sqrt{|m n|} / c) & \text { if } m>0 .\end{cases}
\end{aligned}
$$


When $m=0$, a similar formula holds, and it can be further evaluated to give Cohen's formulas. A modified version holds when $s=1$; see [Bruinier et al. 2006].

Of course, $g_{k,-m}$ can be expressed in terms of the basis elements $f_{k, m}$. If there are no nonzero cusp forms in $M_{2 s}$, then $g_{k,-m}=f_{k, m}$ for all $m$. In general, however,

$$
g_{k,-m}-f_{k, m} \in S_{k}
$$

is a nonzero cusp form. It seems likely that the Fourier coefficients $b_{k}(m, n)$ of $g_{k, m}$ are irrational, even transcendental, in general.

\section{Weak Maass forms}

Next we will show that for $f \in M_{2-2 s}^{!}$with $s \in \mathbb{Z}^{+}$, the function $\partial^{s-1} f$ is a weak Maass form, and we will compute its Fourier expansion. Recall that $\partial^{s-1}$ was defined in (4). Then we express $\partial^{s-1} f_{2-2 s, m}$ in terms of certain Poincaré series. We need the following result which, in essence, is due to Maass; see also [Lewis and Zagier 2001, page 250].

Proposition 3. Suppose $f(\tau)=\sum_{n} a(n) q^{n} \in M_{2-2 s}^{!}$for integral $s \geq 1$. Then $\partial^{s-1} f$ is a weak Maass form for $\Gamma$ with eigenvalue $s(1-s)$. Explicitly, we have

$$
\begin{aligned}
\partial^{s-1} f(\tau)=2 \pi y^{1 / 2} \sum_{n>0} a(-n) n^{s-1 / 2} I_{s-1 / 2}(2 \pi n y) e(-n x) & \\
+(-1)^{s-1}\left(\pi^{1 / 2-s} \Gamma(s-1 / 2) y^{1-s} a(0)\right. & \\
& \left.\quad+2 y^{1 / 2} \sum_{n \neq 0} a(n)|n|^{s-1 / 2} K_{s-1 / 2}(2 \pi|n| y) e(n x)\right),
\end{aligned}
$$

where $I$ and $K$ are the usual Bessel functions.

Proof. By induction it is readily shown that for $n>0$

$$
\partial^{s-1} e(-n \tau)=n^{s-1} \sum_{m=0}^{s-1} \frac{(s-1+m) !}{m !(s-1-m) !}(-4 \pi n y)^{m} e(-n \tau) .
$$

Standard formulas for Bessel functions with half-integral parameter [Gradshteyn and Ryzhik 1994] yield

$$
\begin{aligned}
\partial^{s-1} e(-n \tau) & =2 n^{s-1 / 2} y^{1 / 2}\left(\pi I_{s-1 / 2}(2 \pi n y)+(-1)^{s-1} K_{s-1 / 2}(2 \pi n y)\right) e(n x), \\
\partial^{s-1} e(n \tau) & =2(-1)^{s-1} n^{s-1 / 2} y^{1 / 2} K_{s-1 / 2}(2 \pi n y) e(n x), \\
\partial^{s-1}(1) & =(-1)^{s-1} \pi^{1 / 2-s} \Gamma(s-1 / 2) y^{1-s} .
\end{aligned}
$$

These formulas easily give the stated formula, thus finishing the proof. 
We next express the weak Maass form $\partial^{s-1} f_{2-2 s, m}$ associated to the basis element $f_{2-2 s, m}$ in terms of certain Poincaré series, when $s \geq 2$ and $2 s=12 \ell+k^{\prime}$ as before. For $m \in \mathbb{Z}$ with $m \neq 0$, consider the Poincaré series (see [Niebur 1973])

$$
F_{m}(\tau, s)=2 \pi|m|^{s-1 / 2} \sum_{\gamma \in \Gamma_{\infty} \backslash \Gamma} e(m \operatorname{Re} \gamma \tau)(\operatorname{Im} \gamma \tau)^{1 / 2} I_{s-1 / 2}(2 \pi|m| \operatorname{Im} \gamma \tau),
$$

which converges absolutely for $\operatorname{Re} s>1$. Here $\Gamma_{\infty}$ is the subgroup of translations in $\Gamma$. Clearly $F_{m}(\gamma \tau, s)=F_{m}(\tau, s)$ for $\gamma \in \Gamma$ and $\Delta F_{m}(\tau, s)=s(1-s) F_{m}(\tau, s)$.

Proposition 4. For integral $s \geq 2$, we have for $m \geq \ell+1$

$$
\partial^{s-1} f_{2-2 s, m}(\tau)=F_{-m}(\tau, s)+\sum_{0<n<\ell+1} a_{2-2 s}(m,-n) F_{-n}(\tau, s) .
$$

Proof. We need the Fourier expansion of $F_{m}$. This can be found, for instance, in [Fay 1977]. Let $\xi(s)=\pi^{-s / 2} \Gamma(s / 2) \zeta(s)$. Then we have

$$
\begin{aligned}
F_{m}(\tau, s)=2 \pi|m|^{s-1 / 2} y^{1 / 2} I_{s-1 / 2}(2 \pi|m| y) e(m x)+\frac{4 \pi \sigma_{2 s-1}(|m|)}{(2 s-1) \xi(2 s)} y^{1-s} \\
+4 \pi|m|^{s-1 / 2} \sum_{n \neq 0} c(m, n ; s) y^{1 / 2} K_{s-1 / 2}(2 \pi|n| y) e(n x),
\end{aligned}
$$

where

$$
c(m, n ; s)=\sum_{c>0} c^{-1} K_{0}(m, n ; c) \cdot \begin{cases}I_{2 s-1}\left(4 \pi \sqrt{|m n|} c^{-1}\right) & \text { if } m n<0 \\ J_{2 s-1}\left(4 \pi \sqrt{|m n|} c^{-1}\right) & \text { if } m n>0\end{cases}
$$

and

$$
K_{0}(m, n ; c)=\sum_{a(\bmod c)^{*}} e\left(\frac{m a+n \bar{a}}{c}\right)
$$

is the usual Kloosterman sum, the * restricting the sum to $(a, c)=1$. Consider the Maass form

$$
\phi(\tau)=\partial^{s-1} f_{2-2 s, m}(\tau)-\left(F_{-m}(\tau, s)+\sum_{0<n<\ell+1} a_{2-2 s}(m,-n) F_{-n}(\tau, s)\right) .
$$

By Proposition 3 and (14), we have

$$
\phi(\tau)=c(0) y^{1-s}+\sum_{n \neq 0} c(n) y^{1 / 2} K_{s-1 / 2}(2 \pi|n| y) e(n x),
$$

where each $c(n)$ can be computed explicitly in terms of the $c_{s}(m, n)$ and the $a_{2-2 s}(m, n)$. Since $\phi \in L^{2}(\Gamma \backslash \mathcal{H})$ with eigenvalue $s(1-s)$, it must be equal to 0 .

In the case $s=1$, the Poincaré series $F_{m}(\tau, 1)$ is defined through analytic continuation (see for example [Niebur 1973]), and Proposition 4 continues to hold in the modified form

$$
f_{0, m}(\tau)=j_{m}(\tau)=F_{-m}(\tau, 1)-24 \sigma(m) \quad \text { for } m \geq 1 .
$$




\section{Preliminary formulas for the trace}

For the proof of Theorem 1, we will need to compute the trace of $\partial^{s-1} f_{2-2 s, m}$ in terms of the coefficients of the basis elements $f_{s+1 / 2, m}$. In view of Proposition 4, we are reduced to computing $\operatorname{Tr}_{d, D}\left(F_{m}(\cdot, s)\right)$, where $F_{m}(\tau, s)$ is the Poincaré series defined in (12). When $D=s=1$, it was shown in [Duke 2006] that this trace may be expressed in a simple way in terms of a certain exponential sum. In general we need the exponential sum introduced in [Kohnen 1985]:

$$
S_{m}(d, D ; c)=\sum_{\substack{b(\bmod c) \\ b^{2} \equiv D d(\bmod c)}} \chi\left(\frac{c}{4}, b, \frac{b^{2}-D d}{c}\right) e\left(\frac{2 m b}{c}\right),
$$

where $\chi$ is defined in $(2)$ and $c \equiv 0(\bmod 4)$. Clearly $S_{-m}(d, D ; c)=\overline{S_{m}}(d, D ; c)=$ $S_{m}(d, D ; c)$. We have the following identity.

Proposition 5. Let $s \geq 2$ and $m \neq 0$. Suppose $D$ is fundamental and that $d D<0$. Then

$$
\begin{aligned}
\operatorname{Tr}_{d, D}\left(F_{m}(\cdot, s)\right)=\sqrt{2} \pi|m|^{s-1 / 2}|d|^{1 / 4}|D|^{1 / 4} & \\
& \times \sum_{c \equiv 0(\bmod 4)} c^{-1 / 2} S_{m}(d, D ; c) I_{s-1 / 2}\left(\frac{4 \pi \sqrt{m^{2}|d D|}}{c}\right) .
\end{aligned}
$$

Proof. We have the absolutely convergent expression that

$$
\begin{array}{r}
\operatorname{Tr}_{d, D}\left(F_{m}(\cdot, s)\right)=2 \pi|m|^{s-1 / 2} \sum_{Q} \frac{\chi(Q)}{\omega_{Q}} \sum_{\gamma \in \Gamma_{\infty} \backslash \Gamma} e\left(\begin{array}{r}
\left.\operatorname{Re}_{\gamma} \tau_{Q}\right)\left(\operatorname{Im} \gamma \tau_{Q}\right)^{1 / 2} \\
\times I_{s-1 / 2}\left(2 \pi|m| \operatorname{Im} \gamma \tau_{Q}\right)
\end{array}\right. \\
=\sqrt{2} \pi|m|^{s-\frac{1}{2}}|d|^{1 / 4}|D|^{1 / 4} \sum_{a=1}^{\infty} a^{-1 / 2} I_{s-1 / 2}\left(\frac{\pi \sqrt{m^{2}|d D|}}{a}\right) \\
\times\left[\sum_{Q} \frac{\chi(Q)}{\omega_{Q}} \sum_{\gamma} e\left(m \operatorname{Re}\left(\gamma \tau_{Q}\right)\right)\right],
\end{array}
$$

where the sum over $\gamma$ is over all $\gamma \in \Gamma_{\infty} \backslash \Gamma$ with $\operatorname{Im} \gamma \tau_{Q}=\sqrt{|D d|} /(2 a)$. Consider the sum in brackets in the expression above. For fixed $a>0$, the values of $2 a \operatorname{Re}\left(\gamma \tau_{Q}\right)$ run over the $(\bmod 2 a)$-incongruent solutions to the quadratic congruence $b^{2} \equiv d D(\bmod 4 a)$ with multiplicity $w_{Q}$ as $\gamma$ and $Q$ run over their respective representatives. Thus the term in brackets is equal to $\frac{1}{2} S_{m}(d, D ; 4 a)$. Replacing $4 a$ with $c$ finishes the proof.

We need to express the traces in terms of the Fourier coefficients of modular forms. This is done by applying an identity, originally due to Salié in a special case, to transform the sum of exponential sums in Proposition 5 into a sum of Kloosterman sums. This sum may then be interpreted in terms of the Fourier 
coefficients of half-integral weight Poincaré series. This technique goes back to Zagier [1975], who applied it in the context of base-change. Kohnen [1985] applied it to the Shimura lift of cusp forms. More recently, this method has proved to be fruitful in the context of weakly holomorphic forms. Duke [2006] applied it to give a new proof of Zagier's original identity for traces of singular moduli. The technique has since been extended in various ways in [Jenkins 2006] and [Bringmann and Ono 2007]; in particular, the latter gave the following formula for the trace of $F_{m}(\tau, s)$ in terms of the coefficients $b_{k}(m, n)$ of half-integral weight Poincaré series when $m=-1$ and $(-1)^{s} D<0$.

Proposition 6. Suppose $m \neq 0, s \geq 2$ and $d D<0$ with $D$ fundamental. Then

$$
\begin{aligned}
\operatorname{Tr}_{d, D}\left(F_{m}(\cdot, s)\right)=\varepsilon|d|^{s / 2}|D|^{(1-s / 2)} \sum_{n \mid m} \chi_{D}(n) n^{s-1} b_{s+1 / 2}(-|d|, & \left.\frac{m^{2}|D|}{n^{2}}\right) \\
& \text { if }(-1)^{s} D>0 ;
\end{aligned}
$$

$\operatorname{Tr}_{d, D}\left(F_{m}(\cdot, s)\right)=\varepsilon|d|^{(1-s) / 2}|D|^{s / 2}|m|^{2 s-1} \sum_{n \mid m} \chi_{D}(n) n^{-s} b_{s+1 / 2}\left(\frac{-m^{2}|D|}{n^{2}},|d|\right)$,

$$
\text { if }(-1)^{s} D<0 .
$$

Here the sums $n \mid m$ are over the positive divisors of $m, \varepsilon=(-1)^{\lfloor(s+1) / 2\rfloor}$, and $b_{s+1 / 2}$ was defined in Proposition 2.

Proof. Recall the Kloosterman sum associated to modular forms of half-integral weight defined in (10). It is clear that replacing $k$ with $k+2$ does not change this sum; each $K_{s+1 / 2}(m, n, c)$ is equal to $K_{1 / 2}(m, n ; c)$ or $K_{3 / 2}(m, n ; c)$, depending on whether $s$ is even or odd, respectively. In fact, we have the relations

$$
K_{1 / 2}(m, n ; c)=i \cdot K_{3 / 2}(-m,-n ; c)=K_{1 / 2}(n, m ; c) .
$$

We have the following identity for the Kloosterman sums, which can be proved by a slight modification of the proof of Kohnen [1985, Proposition 5, page 258]; see also [Duke 2006; Jenkins 2006; Tóth 2005].

Lemma 1. For integers $m \neq 0$ and $c>0$ with $4 \mid c$, an integer $d$ with $d \equiv 0,1$ $(\bmod 4)$ and $D$ a fundamental discriminant, we have the identity

$$
S_{m}(d, D ; c)=(1-i) \sum_{n \mid(m, c / 4)}\left(1+\delta_{\text {odd }}(c /(4 n))\right) \chi_{D}(n) \sqrt{\frac{n}{c}} K_{1 / 2}\left(d, m^{2} D / n^{2} ; c / n\right) .
$$

By Proposition 5 and Lemma 1, we quickly derive that

$$
\begin{aligned}
& \operatorname{Tr}_{d, D}\left(F_{m}(\cdot, s)\right)=\sqrt{2} \pi(1-i)|m|^{s-1 / 2}|d|^{1 / 4}|D|^{1 / 4} \sum_{n \mid m} \chi_{D}(n) n^{-1 / 2} \\
& \quad \times \sum_{c \equiv 0(\bmod 4)} c^{-1}\left(1+\delta_{\text {odd }}(c / 4)\right) K_{1 / 2}\left(d, m^{2} D / n^{2} ; c\right) I_{s-1 / 2}\left(\frac{4 \pi}{c} \sqrt{m^{2}|D d| / n^{2}}\right) .
\end{aligned}
$$


Comparison with Proposition 2 and the use of (15) finishes the proof.

\section{The Zagier lift}

In this section we give the proof of Theorem 1. The following proposition gives an explicit formula for the Zagier lift of $f \in M_{2-2 s}^{!}$when $(-1)^{s} D>0$. In its proof we make repeated use of the classical Shimura lift, integral and half-integral weight duality from (7) and (9), and the fact that the constant term of a form in $M_{2}^{!}$must vanish. Write $2 s=12 \ell+k^{\prime}$ with $k^{\prime} \in\{0,4,6,8,10,14\}$ as above.

Proposition 7. Suppose $s \geq 2$ is an integer and $f(\tau)=\sum_{n} a(n) q^{n} \in M_{2-2 s}^{!}$. Suppose $D$ is a fundamental discriminant with $(-1)^{s} D>0$. Then the D-th Zagier lift of $f$ is given by

$$
\mathfrak{Z}_{D} f=\sum_{m>0} a(-m) \sum_{n \mid m} \chi_{D}(n) n^{s-1} f_{3 / 2-s, m^{2}|D| / n^{2}} .
$$

Proof. Recall that when $(-1)^{s} D>0$, the Zagier lift was defined by

$$
\begin{aligned}
\mathfrak{Z}_{D} f(\tau)=\sum_{m>0} a(-m) \sum_{n \mid m} \chi_{D}(n) n^{s-1} q^{-m^{2}|D| / n^{2}} & \\
& +\frac{1}{2} L\left(1-s, \chi_{D}\right) a(0)+\sum_{d: d D<0} \operatorname{Tr}_{d, D}^{*}(f) q^{|d|},
\end{aligned}
$$

where

$$
\operatorname{Tr}_{d, D}^{*}(f)=(-1)^{\lfloor(s-1) / 2\rfloor}|d|^{-s / 2}|D|^{(s-1) / 2} \operatorname{Tr}_{d, D}\left(\partial^{s-1} f\right) .
$$

We prove Proposition 7 by comparing the Fourier coefficients of $\mathfrak{Z}_{D} f$ with those of the function on the right side of (16), which we will denote simply by $F$. We do this separately for the positive coefficients, the principal parts, and the constant terms.

Consider first the positive coefficients. By Propositions 4 and 6 , we have for $m>\ell$ that

$$
\begin{aligned}
-\operatorname{Tr}_{d, D}^{*}\left(f_{2-2 s, m}\right) & =\sum_{n \mid m} \chi_{D}(n) n^{s-1} b_{s+1 / 2}\left(-|d| ; m^{2}|D| / n^{2}\right) \\
+ & \sum_{j=1}^{\ell} a_{2-2 s}(m,-j) \sum_{h \mid j} \chi_{D}(h) h^{s-1} b_{s+1 / 2}\left(-|d| ; j^{2}|D| / h^{2}\right) .
\end{aligned}
$$

From (11), we have the cusp form

$$
C(\tau)=g_{s+1 / 2,-|d|}(\tau)-f_{s+1 / 2,|d|}(\tau)=\sum_{n \geq 1} c(n) q^{n} .
$$

Thus

$$
b_{s+1 / 2}\left(-|d|, j^{2}|D| / h^{2}\right)=a_{s+1 / 2}\left(|d|, j^{2}|D| / h^{2}\right)+c\left(j^{2}|D| / h^{2}\right) .
$$


However, $\mathscr{Y}_{D} C$, the $D$-th Shimura lift of $C$, is a cusp form of weight $2 s$ with $j$-th coefficient $\sum_{h \mid j} \chi_{D}(h) h^{s-1} c\left(j^{2}|D| / h^{2}\right)$. The contribution to $-\operatorname{Tr}_{d, D}^{*}\left(f_{2-2 s, m}\right)$ in (17) from coefficients of $C$, which is

$$
\sum_{n \mid m} \chi_{D}(n) n^{s-1} c\left(m^{2}|D| / n^{2}\right)+\sum_{j=1}^{\ell} a_{2-2 s}(m,-j) \sum_{h \mid j} \chi_{D}(h) h^{s-1} c\left(j^{2}|D| / h^{2}\right),
$$

can be interpreted as the constant term of $\left(\varphi_{D} C\right) f_{2-2 s, m} \in M_{2}^{!}$, which must be zero. Thus we have

$$
\begin{aligned}
-\operatorname{Tr}_{d, D}^{*}\left(f_{2-2 s, m}\right) & =\sum_{n \mid m} \chi_{D}(n) n^{s-1} a_{s+1 / 2}\left(|d|, m^{2}|D| / n^{2}\right) \\
& +\sum_{j=1}^{\ell} a_{2-2 s}(m,-j) \sum_{h \mid j} \chi_{D}(h) h^{s-1} a_{s+1 / 2}\left(|d|, j^{2}|D| / h^{2}\right) .
\end{aligned}
$$

By duality, $\operatorname{Tr}_{d, D}^{*}\left(f_{2-2 s, m}\right)$ is the coefficient of $q^{|d|}$ in the Fourier expansion of

$$
\sum_{n \mid m} \chi_{D}(n) n^{s-1} f_{3 / 2-s, m^{2}|D| / n^{2}}-\sum_{j=1}^{\ell} a_{2 s}(-j, m) \sum_{h \mid j} \chi_{D}(h) h^{s-1} f_{3 / 2-s, j^{2}|D| / h^{2}} .
$$

For an arbitrary form $f=\sum a(m) q^{m} \in M_{2-2 s}^{!}$, we have $f=\sum_{m>\ell} a(-m) f_{2-2 s, m}$, and so

$$
\operatorname{Tr}_{d, D}^{*}(f)=\sum_{m>\ell} a(-m) \operatorname{Tr}_{d, D}^{*}\left(f_{2-2 s, m}\right)
$$

is the coefficient of $q^{|d|}$ in

$$
\begin{aligned}
\sum_{m>\ell} a(-m) & \left(\sum_{n \mid m} \chi_{D}(n) n^{s-1} f_{3 / 2-s, m^{2}|D| / n^{2}}\right. \\
& \left.-\sum_{j=1}^{\ell} a_{2 s}(-j, m) \sum_{h \mid j} \chi_{D}(h) h^{s-1} f_{3 / 2-s, j^{2}|D| / h^{2}}\right) .
\end{aligned}
$$

For $1 \leq j \leq \ell$ we have, once again using that the constant of a form in $M_{2}^{!}$vanishes, that $a(-j)=-\sum_{m>\ell} a(-m) a_{2 s}(-j, m)$. Thus the form in the previous equation simplifies to $F$.

Next consider the principal parts. The properties of the basis elements given in Section 2 show that $f_{3 / 2-s, m^{2}|D| / n^{2}}=0$ if $m^{2}|D| / n^{2}<C$ for some $C$ that depends only on the weight $3 / 2-s$. We use this and the Fourier expansion

$$
f_{3 / 2-s, m^{2}|D| / n^{2}}(\tau)=q^{-m^{2}|D| / n^{2}}+\sum_{h} a_{3 / 2-s}\left(m^{2}|D| / n^{2}, h\right) q^{h}
$$


to write the negative powers of $q$ appearing in the Fourier expansion of $F$ as

$$
\begin{aligned}
& \sum_{m>0} a(-m) \sum_{n \mid m} \chi_{D}(n) n^{s-1} q^{-m^{2}|D| / n^{2}} \\
- & \sum_{m>0} a(-m) \sum_{\substack{n\left|m \\
m^{2}\right| D \mid / n^{2}<C}} \chi_{D}(h) n^{s-1} q^{-m^{2}|D| / n^{2}} \\
+ & \sum_{m, h>0} a(-m) \sum_{n \mid m} \chi_{D}(n) n^{s-1} a_{3 / 2-s}\left(m^{2}|D| / n^{2},-h\right) q^{-h} .
\end{aligned}
$$

The first sum is the principal part of $\mathfrak{Z}_{D} f$, so we must prove that the remaining part, call it $S$, vanishes. By duality,

$$
\begin{aligned}
& S=-\sum_{m>0} a(-m)\left(\sum_{\substack{n\left|m \\
m^{2}\right| D \mid / n^{2}<C}} \chi_{D}(n) n^{s-1} q^{-m^{2}|D| / n^{2}}\right. \\
&\left.+\sum_{h>0} \sum_{n \mid m} \chi_{D}(n) n^{s-1} a_{s+1 / 2}\left(-h, m^{2}|D| / n^{2}\right) q^{-h}\right) .
\end{aligned}
$$

Now for any $h>0$, the coefficient of $q^{m}$ in the Shimura lift $\mathscr{Y}_{D} f_{s+1 / 2,-h}$ of the cusp form $f_{s+1 / 2,-h}$ is given by

$$
\sum_{n \mid m} \chi_{D}(n) n^{s-1} \cdot\left(a_{s+1 / 2}\left(-h, m^{2}|D| / n^{2}\right)+\left\{\begin{array}{ll}
1 & \text { if } m^{2}|D| / n^{2}=h, \\
0 & \text { otherwise. }
\end{array}\right) .\right.
$$

(The last term here arises from the initial $q^{h}$ in the Fourier expansion of $f_{s+1 / 2,-h}$, since $a_{s+1 / 2}(-h, h)$ is zero by definition.) From this, it is clear that the coefficient of $q^{-h}$ in $S$ for each $h>0$ can be interpreted as the constant term of $\left(\mathscr{Y}_{D} f_{s+1 / 2,-h}\right) f \in M_{2}^{!}$, so $S=0$.

Finally we evaluate the constant term of $F$, again using duality, as

$$
\begin{aligned}
\sum_{m>0} a(-m) \sum_{n \mid m} \chi_{D}(n) n^{s-1} a_{3 / 2-s}\left(m^{2}|D| / n^{2}, 0\right) & \\
& =-\sum_{m>0} a(-m) \sum_{n \mid m} \chi_{D}(n) n^{s-1} a_{s+1 / 2}\left(0, m^{2}|D| / n^{2}\right) .
\end{aligned}
$$

Since $s \geq 2$, we have by [Kohnen and Zagier 1981]

$$
\mathscr{Y}_{D} f_{s+1 / 2,0}(\tau)=\frac{1}{2} L\left(1-s, \chi_{D}\right)+\sum_{m>0}\left(\sum_{n \mid m} \chi_{D}(n) h^{s-1} a_{s+1 / 2}\left(0, n^{2}|D| / h^{2}\right)\right) q^{m},
$$

and the constant term of $\left(\mathscr{Y}_{D} f_{s+1 / 2,0}\right) f \in M_{2}^{!}$is

$$
\frac{1}{2} L\left(1-s, \chi_{D}\right) a(0)+\sum_{m>0} a(-m)\left(\sum_{n \mid m} \chi_{D}(n) n^{s-1} a_{s+1 / 2}\left(0, m^{2}|D| / n^{2}\right)\right)=0 .
$$


We also need the corresponding statement if $(-1)^{s} D<0$.

Proposition 8. Suppose $s \geq 2$ is an integer and $f \in M_{2-2 s}^{!}$has Fourier coefficients $a(n)$. Suppose $D$ is a fundamental discriminant with $(-1)^{s} D<0$. Then the $D$-th Zagier lift of $f$ is given by

$$
\mathfrak{Z}_{D} f=\sum_{m>0} a(-m) m^{2 s-1} \sum_{n \mid m} \chi_{D}(n) n^{-s} f_{s+1 / 2, m^{2}|D| / n^{2}}+g,
$$

where $g \in S_{s+1 / 2}$ is the unique cusp form whose Fourier coefficients $b(n)$ match those of $\mathfrak{Z}_{D} f$ for the first $\ell$ positive values of $n$ with $(-1)^{s} n \equiv 0,1(\bmod 4)$.

Proof. Using Propositions 4 and 6 as before, we find that

$$
\begin{array}{r}
\operatorname{Tr}_{d, D}^{*}\left(f_{2-2 s, m}\right)=\sum_{j=1}^{\ell} a_{2-2 s}(m,-j) j^{2 s-1} \sum_{h \mid j} \chi_{D}(h) h^{-s} b_{s+1 / 2}\left(-j^{2}|D| / h^{2} ;|d|\right) \\
+m^{2 s-1} \sum_{n \mid m} \chi_{D}(n) n^{-s} b_{s+1 / 2}\left(-m^{2}|D| / n^{2} ;|d|\right) .
\end{array}
$$

Thus an arbitrary form $f=\sum a(m) q^{m}=\sum_{m>\ell} a(-m) f_{2-2 s, m}$ has trace $\operatorname{Tr}_{d, D}^{*}(f)$ given by

$$
\begin{aligned}
\sum_{m>\ell} a & (-m)\left(m^{2 s-1} \sum_{n \mid m} \chi_{D}(n) n^{-s} b_{s+1 / 2}\left(-m^{2}|D| / n^{2} ;|d|\right)\right. \\
+ & \left.\sum_{j=1}^{\ell} a_{2-2 s}(m,-j) j^{2 s-1} \sum_{h \mid j} \chi_{D}(h) h^{-s} b_{s+1 / 2}\left(-j^{2}|D| / h^{2} ;|d|\right)\right) \\
& =\sum_{m>0} a(-m) m^{2 s-1} \sum_{n \mid m} \chi_{D}(n) n^{-s} b_{s+1 / 2}\left(-m^{2}|D| / n^{2} ;|d|\right)
\end{aligned}
$$

where we have simplified as before. This is just the coefficient of $q^{|d|}$ in the modular form $F \in M_{s+1 / 2}^{!}$given by

$$
F=\sum_{m>0} a(-m) m^{2 s-1} \sum_{n \mid m} \chi_{D}(n) n^{-s} g_{s+1 / 2,-m^{2}|D| / n^{2}}
$$

Now since $g_{s+1 / 2,-m^{2}|D| / n^{2}}-f_{s+1 / 2, m^{2}|D| / n^{2}} \in S_{s+1 / 2}$ from (11), we find that

$$
F=\sum_{m>0} a(-m) m^{2 s-1} \sum_{n \mid m} \chi_{D}(n) n^{-s} f_{s+1 / 2, m^{2}|D| / n^{2}}+g
$$

for a certain cusp form $g$, and, arguing as in Proposition 7 , the principal part of $F$ matches the principal part of $\mathfrak{Z}_{D} f$. Since the constant term and positive coefficients of $F$ match those of $\mathfrak{Z}_{D} f$, Proposition 8 now follows. 
The first statement of Theorem 1 follows from Propositions 7 and 8 . The statement on integrality follows from Proposition 1 in the case $(-1)^{s} D>0$. Otherwise we can reduce to this case using the following identity, which holds if $(-1)^{s} D<0$ and $D^{\prime}$ is fundamental with $(-1)^{s} D^{\prime}>0$ :

$$
\operatorname{Tr}_{m^{2} D^{\prime}, D}^{*}(f)=-m^{2 s-1} \sum_{a \mid m} \mu(a) \chi_{D^{\prime}}(a) \sum_{b \mid m a^{-1}} \chi_{D}(b)(a b)^{-s} \operatorname{Tr}_{(m / a b)^{2} D, D^{\prime}}^{*}(f) .
$$

This identity is a consequence of the following lemma.

Lemma 2. For $D$ and $D^{\prime}$ fundamental discriminants with $D D^{\prime}<0$ and $m \in \mathbb{Z}^{+}$,

$$
\operatorname{Tr}_{m^{2} D^{\prime}, D}=\sum_{a \mid m} \mu(a) \chi_{D^{\prime}}(a) \sum_{b \mid m a^{-1}} \chi_{D}(b) \operatorname{Tr}_{(m / a b)^{2} D, D^{\prime}}
$$

Lemma 2 is obtained by writing the trace as a sum of sums over primitive quadratic forms, noting that $\chi_{D}=\chi_{D^{\prime}}$ for such primitive forms, and applying Möbius inversion.

We now briefly indicate how one shows that the Zagier lift is compatible with the Hecke operators. If $k \in 2 \mathbb{Z}>0$ and $p$ is a prime, the weight $k$ Hecke operator $\left.\right|_{k} T(p)$ acts on a modular form $f(\tau)=\sum_{n} a(n) q^{n} \in M_{k}^{!}$by

$$
\left.f\right|_{k} T(p)=\sum_{n}\left(a(p n)+p^{k-1} a(n / p)\right) q^{n} .
$$

If $k \in 2 \mathbb{Z} \leq 0$, we multiply this by $p^{1-k}$ so that $\left.\right|_{k} T(p)$ preserves the integrality of Fourier coefficients.

When $0<s \in \mathbb{Z}$, the half-integral weight Hecke operator $\left.\right|_{s+1 / 2} T\left(p^{2}\right)$ acts on a form $g(\tau)=\sum_{n} b(n) q^{n} \in M_{s+1 / 2}^{!}$by

$$
\left.g\right|_{s+1 / 2} T\left(p^{2}\right)=\sum_{n}\left(b\left(p^{2} n\right)+\left((-1)^{s} n / p\right) p^{s-1} b(n)+p^{2 s-1} b\left(n / p^{2}\right)\right) q^{n} .
$$

Again, for $s \leq 0$, we normalize this by multiplying by $p^{1-2 s}$.

It is straightforward to see that $\left.\left(\mathfrak{Z}_{D} f\right)\right|_{3 / 2-\hat{s}} T\left(p^{2}\right)=\mathfrak{Z}_{D}\left(\left.f\right|_{2-2 s} T(p)\right)$ for any prime $p$. In the case that $(-1)^{s} D>0$, we need only use the explicit Fourier expansion of the Zagier lift to compare principal parts. If $(-1)^{s} D<0$, though, we must also show that

$$
\begin{array}{r}
\operatorname{Tr}_{(-1)^{s} n, D}^{*}\left(\left.f\right|_{2-2 s} T(p)\right)=\operatorname{Tr}_{(-1)^{s} n p^{2}, D}^{*}(f)+\left((-1)^{s} n / p\right) p^{s-1} \operatorname{Tr}_{(-1)^{s} n, D}^{*}(f) \\
+p^{2 s-1} \operatorname{Tr}_{(-1)^{s} n / p^{2}, D}^{*}(f)
\end{array}
$$

for the first $\ell$ positive values of $n$ with $(-1)^{s} n \equiv 0,1(\bmod 4)$. To see that this holds, we argue as in the proof of [Zagier 2002, Theorem 5(ii)] to show that 
$\operatorname{Tr}_{(-1)^{s} n, D}\left(\left.\left(\partial^{s-1} f\right)\right|_{0} T(p)\right)$ equals

$$
\operatorname{Tr}_{(-1)^{s} n p^{2}, D}\left(\partial^{s-1} f\right)+\left((-1)^{s} n / p\right) \operatorname{Tr}_{(-1)^{s} n, D}\left(\partial^{s-1} f\right)+p \operatorname{Tr}_{(-1)^{s} n / p^{2}, D}\left(\partial^{s-1} f\right),
$$

and we use the fact that if $k<0$, then $\partial_{k}\left(\left.f\right|_{k} T(p)\right)=\left.p \cdot\left(\partial_{k} f\right)\right|_{k+2} T(p)$ to obtain Equation (21).

\section{The Shimura lift}

In this final section we prove Theorem 2. For this we need two propositions:

Proposition 9. Suppose $s \in \mathbb{Z}^{+}$and $\tau \in \mathcal{H}$. As a function of $z \in \mathcal{H}$,

$$
\partial^{s-1}\left(\frac{f_{2 s}(z) f_{2-2 s}(\tau)}{j(\tau)-j(z)}\right)
$$

is a meromorphic modular form of weight $2 s$ with poles of order at most $s$ that only occur at points equivalent to $\tau$ under $\Gamma$.

Proof. Observe first that if $f$ has weight $k$ and $g$ has weight 0 then Equation (3) gives $\partial_{k}(f g)=g \partial_{k}(f)+f \mathcal{D}(g)$. Apply this repeatedly with $g(\tau)=(j(\tau)-$ $j(z))^{-n}$ for $1 \leq n<s$. We derive that

$$
\partial^{s-1}\left(\frac{f_{2-2 s}(\tau)}{j(\tau)-j(z)}\right)=\sum_{n=1}^{s} \frac{g_{n}(\tau)}{(j(z)-j(\tau))^{n}} \quad \text { for } g_{n} \in M_{0}^{!},
$$

from which the result follows easily.

Theorem 2 is a consequence of Proposition 9 together with the following explicit formula for the $D$-th Shimura lift of $f_{s+1 / 2,|d|}$. Write $2 s=12 \ell+k^{\prime}$ as above.

Proposition 10. Suppose $s \geq 2,(-1)^{s} D>0$ and $d D<0$. Then

$$
\mathscr{Y}_{D} f_{s+1 / 2,|d|}(z)=\operatorname{Tr}_{d, D}^{*}\left(\frac{f_{2 s}(z) f_{2-2 s}(\tau)}{j(\tau)-j(z)}\right)+f(z),
$$

where $f \in M_{2 s}$ is the unique holomorphic form whose Fourier coefficients a $(n)$ match those of $\mathscr{Y}_{D} f_{s+1 / 2,|d|}$ for $n=0, \ldots, \ell$.

Proof. By (5) we have, writing $r=e(z)$,

$$
\begin{aligned}
\mathscr{S}_{D} f_{s+1 / 2,|d|}(z)=\frac{1}{2} L\left(1-s, \chi_{D}\right) & a_{s+1 / 2}(|d|, 0) \\
& +\sum_{m>0}\left(\sum_{n \mid m} \chi_{D}(n) n^{s-1} a_{s+1 / 2}\left(|d|, m^{2}|D| / n^{2}\right)\right) r^{m} .
\end{aligned}
$$


By this and (18) we have

$$
\begin{array}{r}
-\sum_{m>\ell} \operatorname{Tr}_{d, D}^{*}\left(f_{2-2 s, m}\right) r^{m}=\mathscr{Y}_{D} f_{s+1 / 2,|d|}(z)-\frac{1}{2} L\left(1-s, \chi_{D}\right) a_{s+1 / 2}(|d|, 0) \\
-\sum_{0<m \leq \ell}\left(\sum_{n \mid m} \chi_{D}(n) n^{s-1} a_{s+1 / 2}\left(|d|, m^{2}|D| / n^{2}\right)\right) r^{m} \\
+\left[\sum_{j=1}^{\ell} \sum_{m>\ell} a_{2-2 s}(m,-j) r^{m} \sum_{h \mid j} \chi_{D}(h) h^{s-1} a_{s+1 / 2}\left(|d|, j^{2}|D| / h^{2}\right)\right] .
\end{array}
$$

Using integral weight duality (7), the bracketed term equals

$$
-\sum_{j=1}^{\ell}\left(f_{2 s,-j}(z)-r^{j}\right) \sum_{h \mid j} \chi_{D}(h) h^{s-1} a_{s+1 / 2}\left(|d|, j^{2}|D| / h^{2}\right),
$$

so the previous equation, after some cancellation, becomes

$$
-\sum_{m>\ell} \operatorname{Tr}_{d, D}^{*}\left(f_{2-2 s, m}\right) r^{m}=\mathscr{Y}_{D} f_{s+1 / 2,|d|}(z)-f(z) .
$$

Then this and (6) imply the claimed identity, at least when $\operatorname{Im} z>\max _{Q} \operatorname{Im} \tau_{Q}$. The full result now follows by analytic continuation.

\section{Acknowledgments}

After this paper was written, we learned that results similar to some of those presented here were obtained independently in [Miller and Pixton 2007]. We thank the referee for some helpful comments.

\section{Appendix}

The table below gives explicit formulas for the first two basis elements $f_{b+1 / 2}$ and $f_{b+1 / 2}^{*}$ of weight $b+1 / 2$ for various $b$ as polynomials in the weight $1 / 2$ theta function $\theta=\sum_{n \in \mathbb{Z}} q^{n^{2}}$ and the weight 2 Eisenstein series on $\Gamma_{0}(4)$ given by $F(z)=$ $\sum_{n=0}^{\infty} \sigma(2 n+1) q^{2 n+1}$. Both $\theta$ and $F$ have integral Fourier coefficients.

The space of holomorphic modular forms on $\Gamma_{0}(4)$ of weight $s+1 / 2$ is generated by the forms $F^{n} \theta^{2 s+1-4 n}$, where $0 \leq n \leq\lfloor(2 s+1 / 4)\rfloor$; see [Cohen 1975]. Thus, in order to construct these basis elements we examine the Fourier expansion of the form $f=\sum_{n=0}^{\lfloor(2 s+1) / 4\rfloor} A(n) F^{n} \theta^{2 s+1-4 n}$ and choose the coefficients $A(n)$ so that $f$ is in the plus space $M_{s+1 / 2}^{!}$and has the appropriate leading terms in its Fourier expansion. The table shows that all of the $A(n)$ are integral for the first two basis elements of each half-integral weight, so it follows that all of the $f_{b+1 / 2}$ and $f_{b+1 / 2}^{*}$ have integral Fourier coefficients. 


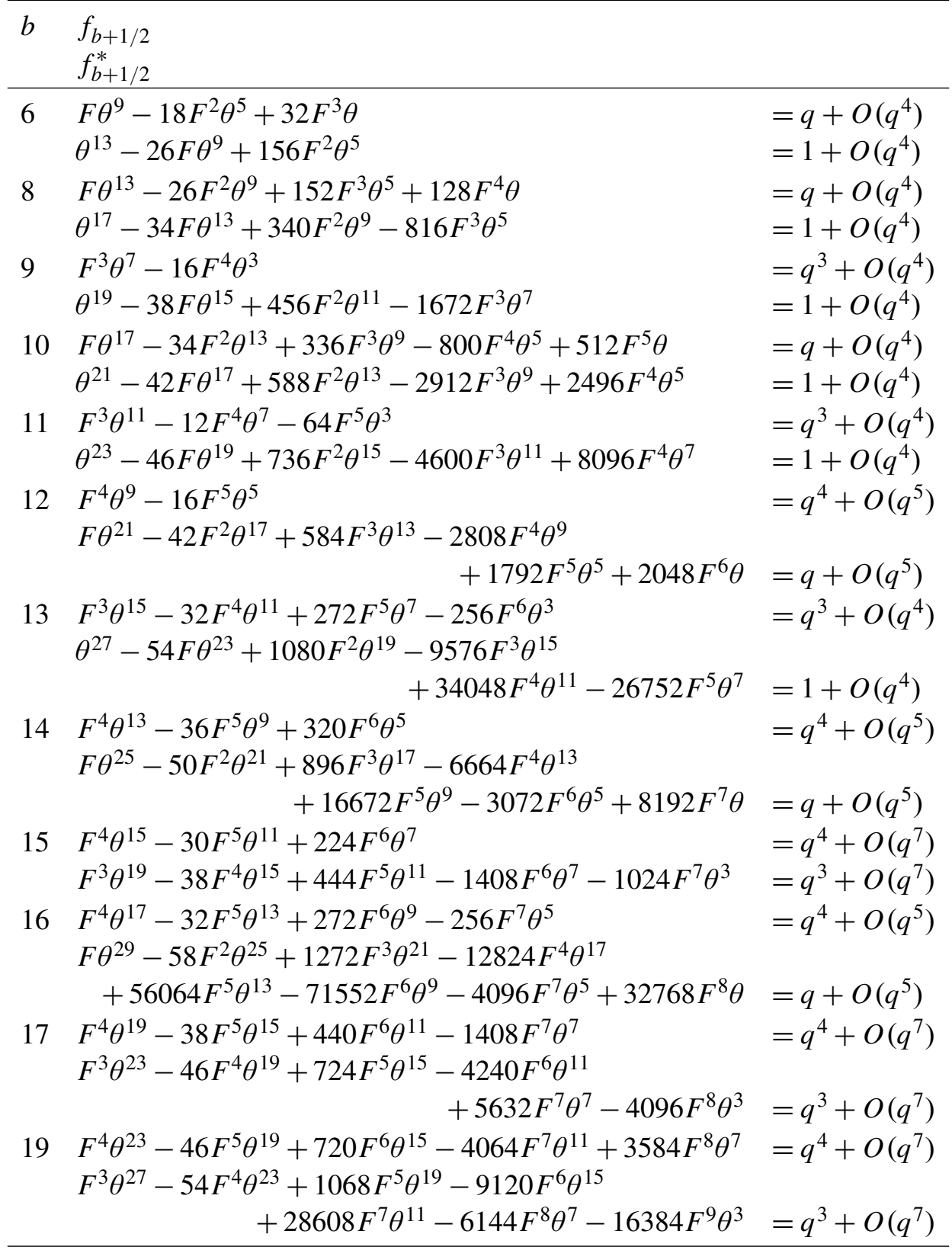

\section{References}

[Borcherds 1995] R. E. Borcherds, "Automorphic forms on $\mathrm{O}_{s+2,2}(\mathbf{R})$ and infinite products", Invent. Math. 120:1 (1995), 161-213. MR 96j:11067 Zbl 0932.11028

[Borcherds 1998] R. E. Borcherds, “Automorphic forms with singularities on Grassmannians", Invent. Math. 132:3 (1998), 491-562. MR 99c:11049 Zbl 0919.11036 
[Bringmann and Ono 2007] K. Bringmann and K. Ono, "Arithmetic properties of coefficients of half-integral weight Maass-Poincaré series”, Math. Ann. 337:3 (2007), 591-612. MR 2007m:11064 Zbl 05145027

[Bruinier and Funke 2006] J. H. Bruinier and J. Funke, "Traces of CM values of modular functions", J. Reine Angew. Math. 594 (2006), 1-33. MR 2007e:11050 Zbl 1104.11021

[Bruinier et al. 2006] J. H. Bruinier, P. Jenkins, and K. Ono, "Hilbert class polynomials and traces of singular moduli”, Math. Ann. 334:2 (2006), 373-393. MR 2007b:11055 Zbl 05013666

[Bruinier et al. 2008] J. H. Bruinier, G. van der Geer, G. Harder, and D. Zagier, The 1-2-3 of modular forms: Lectures at a summer school in Nordfjordeid, Norway, Springer, Berlin, 2008. Zbl 05189476

[Cohen 1975] H. Cohen, "Sums involving the values at negative integers of $L$-functions of quadratic characters", Math. Ann. 217:3 (1975), 271-285. MR 52 \#3080 Zbl 0311.10030

[Duke 2006] W. Duke, "Modular functions and the uniform distribution of CM points", Math. Ann. 334:2 (2006), 241-252. MR 2007e:11051 Zbl 05013661

[Duke and Jenkins 2008] W. Duke and P. Jenkins, "On the zeros and coefficients of certain weakly holomorphic modular forms", Pure Appl. Math. Q. 4:4 (2008), 1327-1340.

[Fay 1977] J. D. Fay, "Fourier coefficients of the resolvent for a Fuchsian group", J. Reine Angew. Math. 293/294 (1977), 143-203. MR 58 \#21944 Zbl 0352.30012

[Funke 2007] J. Funke, "CM points and weight 3/2 modular forms", pp. 107-127 in Analytic number theory: A tribute to Gauss and Dirichlet, edited by W. Duke and Y. Tschinkel, Clay Math. Proc. 7, Amer. Math. Soc., Providence, RI, 2007. MR 2362196 Zbl 05233958

[Gradshteyn and Ryzhik 1994] I. S. Gradshteyn and I. M. Ryzhik, Table of integrals, series, and products, 5th ed., Academic, Boston, 1994. MR 94g:00008 Zbl 0918.65002

[Jenkins 2006] P. Jenkins, "Kloosterman sums and traces of singular moduli”, J. Number Theory 117:2 (2006), 301-314. MR 2006k:11079 Zbl 05023183

[Kohnen 1980] W. Kohnen, "Modular forms of half-integral weight on $\Gamma_{0}(4)$ ", Math. Ann. 248:3 (1980), 249-266. MR 81j:10030 Zbl 0416.10023

[Kohnen 1985] W. Kohnen, "Fourier coefficients of modular forms of half-integral weight", Math. Ann. 271:2 (1985), 237-268. MR 86i:11018 Zbl 0542.10018

[Kohnen and Zagier 1981] W. Kohnen and D. Zagier, "Values of $L$-series of modular forms at the center of the critical strip", Invent. Math. 64:2 (1981), 175-198. MR 83b:10029 Zbl 0468.10015

[Lewis and Zagier 2001] J. Lewis and D. Zagier, "Period functions for Maass wave forms, I", Ann. of Math. (2) 153:1 (2001), 191-258. MR 2003d:11068 Zbl 1061.11021

[Masser 1975] D. Masser, Elliptic functions and transcendence, Lecture Notes in Mathematics 437, Springer, Berlin, 1975. MR 52 \#296 Zbl 0312.10023

[Miller and Pixton 2007] A. Miller and A. Pixton, "Arithmetic traces of non-holomorphic modular invariants", Preprint, 2007, Available at http://www.math.wisc.edu/ ono/reu06zagier.pdf.

[Niebur 1973] D. Niebur, "A class of nonanalytic automorphic functions", Nagoya Math. J. 52 (1973), 133-145. MR 49 \#2557 Zbl 0288.10010

[Petersson 1950] H. Petersson, "Konstruktion der Modulformen und der zu gewissen Grenzkreisgruppen gehörigen automorphen Formen von positiver reeller Dimension und die vollständige Bestimmung ihrer Fourierkoeffizienten", S.-B. Heidelberger Akad. Wiss. Math.-Nat. Kl. 1950 (1950), 417-494. MR 12,806e Zbl 0041.41602

[Ramanujan 1914] S. Ramanujan, "Modular equations and approximations to $\pi$ ", Quart. J. Math. 45 (1914), 350-372. reprinted pp. 23-39 in his Collected papers, AMS/Chelsea, Providence, RI, 2000. MR 2280849 
[Siegel 1969] C. L. Siegel, "Berechnung von Zetafunktionen an ganzzahligen Stellen”, Nachr. Akad. Wiss. Göttingen Math.-Phys. Kl. II 1969 (1969), 87-102. Translated as pp. 249-268 of Advanced analytic number theory, Studies in Mathematics 9, Tata Institute of Fundamental Research, Mumbai, 1980.

[Tóth 2005] Á. Tóth, "On the evaluation of Salié sums”, Proc. Amer. Math. Soc. 133:3 (2005), 643645. MR 2005h:11177 Zbl 02120767

[Zagier 1975] D. Zagier, "Modular forms associated to real quadratic fields", Invent. Math. 30:1 (1975), 1-46. MR 52 \#3062 Zbl 0308.10014

[Zagier 2002] D. Zagier, “Traces of singular moduli”, pp. 211-244 in Motives, polylogarithms and Hodge theory, I (Irvine, CA, 1998), edited by F. Bogomolov and L. Katzarkov, Int. Press Lect. Ser. 3, Int. Press, Somerville, MA, 2002. MR 2004h:11037 Zbl 1048.11035

Communicated by Peter Sarnak

Received 2007-12-03 Revised 2008-05-15 Accepted 2008-06-13

wdduke@ucla.edu

UCLA Mathematics Department, Box 951555, Los Angeles, CA 90095-1555, United States http://www.math.ucla.edu/ wdduke/

jenkins@math.ucla.edu

UCLA Mathematics Department, Box 951555, Los Angeles, CA 90095-1555, United States http://www. math.ucla.edu/ jenkins/ 


\title{
Le défaut d'approximation forte pour les groupes algébriques commutatifs
}

\author{
David Harari
}

\begin{abstract}
On établit une suite exacte décrivant l'adhérence des points rationnels d'un 1motif dans ses points adéliques. On en déduit ensuite que le défaut d'approximation forte pour un groupe algébrique commutatif $G$ est essentiellement mesuré par son groupe de Brauer algébrique via l'obstruction de Brauer-Manin entière.

We give an exact sequence describing the closure of the set of rational points of a 1-motive in its adelic points. From this we deduce that for a commutative algebraic group, the defect of strong approximation is essentially controlled by its algebraic Brauer group, by means of the integral Brauer-Manin obstruction.
\end{abstract}

\section{Introduction}

1.1. Rappels et notations. Soit $M=[Y \rightarrow G]$ un 1-motif sur un corps de nombres $k$, dont on note $M^{*}$ le 1-motif dual. Rappelons (cf. [Harari et Szamuely 2005], section 1) qu'ici $Y$ est un $k$-schéma en groupes (placé en degré -1) localement isomorphe pour la topologie étale à $\mathbf{Z}^{r}$ (avec $r \geq 0$ ) et $G$ est une $k$-variété semiabélienne (extension d'une $k$-variété abélienne $A$ par un $k$-tore $T$ ) que l'on place en degré zéro. Le cas particulier le plus significatif pour cet article est celui où $M=T \simeq[0 \rightarrow T]:$ alors $M^{*}=\widehat{T}[1]=[\widehat{T} \rightarrow 0]$, où $\widehat{T}$ est le module des caractères du tore $T$. Pour simplifier les notations, on note simplement $H^{i}(k, M)$ les groupes d'hypercohomologie $\mathbf{H}^{i}(k, M)$ du 1-motif $M$; si par exemple $M=T$, on a donc $H^{i}(k, M)=H^{i}(k, T)$ et $H^{i}\left(k, M^{*}\right)=H^{i}(k, \widehat{T}[1])=H^{i+1}(k, \widehat{T})$.

Soit $\Omega$ l'ensemble de toutes les places de $k$. On note 0 l'anneau des entiers de $k$ et si $v$ est une place non archimédienne de $k$, on note $O_{v}$ l'anneau des entiers du complété $k_{v}$ de $k$ en $v$. Si $S$ est un ensemble fini de places de $k$ (contenant les places archimédiennes), on note $\mathrm{O}_{S}$ le sous-anneau de $k$ constitué des entiers en

MSC2000: primary 14L15; secondary 12G05, $11 \mathrm{G} 09$.

Mots-clefs: approximation forte, groupe de Brauer, 1-motif, strong approximation, Brauer group, 1-motive. 
dehors de $S$. On note aussi $\mathbf{A}_{k}$ le groupe des adèles de $k$ et

$$
\mathbf{A}_{\odot}:=\prod_{v \in \Omega} \mathfrak{O}_{v}
$$

le groupe des adèles entiers de $k$ puis

$$
\mathbf{A}_{S}=\prod_{v \in S} k_{v} \times \prod_{v \notin S} \mathrm{O}_{v}
$$

le groupe des adèles entiers en dehors de $S$, avec la convention que $O_{v}=k_{v}$ si $v$ est une place archimédienne.

On choisit un ouvert non vide $U=\operatorname{Spec}\left(\mathcal{O}_{S}\right)$ de Spec $O$ tel que $M$ s'étende en un 1-motif $\mathcal{M}=[\mathscr{Y} \rightarrow \mathscr{G}]$ au-dessus de $U$. On note $A$ et $T$ (resp. $\mathscr{A}$ et $\mathscr{T}$ ) la $k$-variété abélienne et le $k$-tore correspondant à $G$ (resp. le $U$-schéma abélien et le $U$-tore correspondant à $\mathscr{G})$.

On note $\mathbf{P}^{0}(M)$ (resp. $\left.\mathbf{P}_{S}^{0}(M)\right)$ le produit restreint des $H^{0}\left(k_{v}, M\right)$ (resp. des $H^{0}\left(k_{v}, M\right)$ pour $v$ non dans $\left.S\right)$, relativement aux $H^{0}\left(\mathcal{O}_{v}, \mathcal{M}\right)$, avec la convention que pour $v$ archimédienne, $H^{0}\left(\mathrm{O}_{v}, M\right)=H^{0}\left(k_{v}, M\right)$ désigne le groupe modifié $\widehat{H}^{0}\left(k_{v}, M\right)$ de Tate défini dans [Harari et Szamuely 2005], p. 103, et aussi que pour $v \in S$ finie, $H^{0}\left(\mathcal{O}_{v}, M\right)$ signifie $H^{0}\left(k_{v}, M\right)$. On pose aussi $\mathscr{P}_{S}^{0}(M):=$ $\prod_{v \in S} H^{0}\left(k_{v}, M\right) \times \prod_{v \notin S} H^{0}\left(\mathcal{O}_{v}, M\right)$. Ainsi $\mathscr{P}_{S}^{0}(M)$ est un sous-groupe de $\mathbf{P}^{0}(M)$, dont la projection sur le produit restreint "tronqué" $\mathbf{P}_{S}^{0}(M)$ n'est autre que

$$
\prod_{v \notin S} H^{0}\left(\mathcal{O}_{v}, M\right)
$$

On appelle $\amalg^{1}(M)$ le noyau de l'application diagonale

$$
H^{1}(k, M) \rightarrow \prod_{v \in \Omega} H^{1}\left(k_{v}, M\right)
$$

En particulier $\amalg(A):=\amalg^{1}(A)$ est le groupe de Tate-Shafarevitch de la variété abélienne $A$. Il est fortement conjecturé que ce groupe est toujours fini. On note aussi $\amalg_{\omega}^{1}(M)$ le sous-groupe de $H^{1}(k, M)$ constitué des éléments dont la restriction à $H^{1}\left(k_{v}, M\right)$ est nulle pour presque toute place $v$.

Pour tout groupe topologique $B$, on note $B^{\wedge}$ le complété de $B$ pour la topologie induite par les sous-groupes ouverts d'indice fini. En particulier si $B$ est discret, alors $B^{\wedge}$ est simplement la complétion profinie de $B$ (et c'est également le cas pour $B=H^{0}\left(k_{v}, M\right)$ avec $v \in \Omega$ via [Harari et Szamuely 2005], remarque 2.4). On note également $B^{D}:=\operatorname{Hom}_{\text {cont }}(B, \mathbf{Q} / \mathbf{Z})$ le dual de $B$. Si $B$ est compact et complètement discontinu (i.e. profini), on a $B^{\wedge}=B$.

Si $X$ est une $k$-variété algébrique gémétriquement intègre, on note $\operatorname{Br} X$ son groupe de Brauer cohomologique, et on pose $\operatorname{Br}_{1} X=\operatorname{ker}[\operatorname{Br} X \rightarrow \operatorname{Br} \bar{X}]$, où $\bar{X}=$ $X \times{ }_{k} \bar{k}(\bar{k}$ désignant une clôture algébrique de $k$ ). 
1.2. Enoncé des résultats principaux. Notre premier objectif est d'établir une suite exacte dans l'esprit du théorème 1.2 de [Harari et Szamuely 2008] (lequel, dans le cas des tores, remonte à [Sansuc 1981]), mais qui fait intervenir une obstruction à l'approximation forte au lieu de l'approximation faible. Plus précisément, on a (voir théorème 2) :

Theorème. Soit $M$ un 1-motif sur $k$, de variété abélienne associée A. On suppose que $\amalg(A)$ est fini et on note $\overline{H^{0}(k, M)}$ l'adhérence de $H^{0}(k, M)$ dans le produit restreint $\mathbf{P}^{0}(M)$. Alors on a une suite exacte

$$
0 \rightarrow \overline{H^{0}(k, M)} \rightarrow \mathbf{P}^{0}(M) \rightarrow H^{1}\left(k, M^{*}\right)^{D} \rightarrow \amalg^{1}(M) \rightarrow 0
$$

La flèche $\mathbf{P}^{0}(M) \rightarrow H^{1}\left(k, M^{*}\right)^{D}$ vient de la dualité locale ([Harari et Szamuely 2005], section 2) et la flèche $H^{1}\left(k, M^{*}\right)^{D} \rightarrow \amalg^{1}(M)$ de la dualité entre les groupes finis $\amalg^{1}(M)$ et $\amalg^{1}\left(M^{*}\right)$ ([Harari et Szamuely 2005], corollaire 4.9). Ainsi l'obstruction à la propriété d'approximation forte pour le 1-motif $M$ est mesurée par le groupe $\operatorname{ker}\left[H^{1}\left(k, M^{*}\right)^{D} \rightarrow \amalg^{1}(M)\right]$ (qui est d'indice fini dans $H^{1}\left(k, M^{*}\right)^{D}$ ). Le cas $M=\mathbf{G}_{m}$ est très proche d'un énoncé classique de théorie du corps de classes (voir [Neukirch et al. 2008], corollaire 8.3.21 page 464) mais dans le cas d'un tore quelconque, l'énoncé semble déjà nouveau. Nous en établirons également une variante permettant d'obtenir un énoncé d'approximation pour les tores (théorème 3) un peu similaire aux résultats de la section 4 de [Naumann 2008].

Dans la dernière section de ce texte, nous démontrerons le résultat suivant (voir théorème 4), qui fait le lien entre l'obstruction de Manin entière (introduite dans [Colliot-Thélène et Fei 2007], voir la section 4 pour plus de détails) et l'approximation forte pour une variété semi-abélienne.

Theorème. Soit $\mathscr{X}$ un $\mathbb{O}$-schéma plat dont la fibre générique $X$ est un espace principal homogène sous une variété semi-abélienne $G$. On suppose que le groupe de Tate-Shafarevitch $\amalg(A)$ du quotient abélien A de G est fini.

Soit $S$ un ensemble fini de places de $k$, contenant les places archimédiennes. On suppose qu'il existe un point adélique $\left(P_{v}\right) \in \mathscr{L}\left(\mathbf{A}_{S}\right)$ qui est orthogonal à $\mathrm{Br}_{1} X$ pour l'accouplement de Brauer-Manin. Alors il existe un point de $\mathscr{L}\left(\mathrm{O}_{S}\right)$ qui est arbitrairement proche de $P_{v}$ pour $v \in S$ non archimédienne, et dans la même composante connexe de $X\left(k_{v}\right)$ que $P_{v}$ pour v réelle. En particulier, si on suppose de plus $\left(P_{v}\right) \in \mathscr{L}\left(\mathbf{A}_{\odot}\right)$, alors $\mathscr{L}(\mathcal{O}) \neq \varnothing$.

Notons que l'énoncé analogue pour l'approximation faible est démontré dans [Harari 2006] (voir aussi [Harari et Szamuely 2008], théorème 6.1.).

\section{Une suite exacte de type Cassels-Tate associée à un 1-motif}

Dans toute cette section, les notations sont celles du début de 1.1. 
Lemme 1. Soit $v \notin S$. On a $H^{1}\left(\mathcal{O}_{v}, \mathscr{G}\right)=0$. Pour $i=0$ ou $i=1$, les applications $H^{i}\left(\mathrm{O}_{v}\right.$, Y $) \rightarrow H^{i}\left(k_{v}, Y\right)$ (induites par l'inclusion $\left.\mathrm{O}_{v} \rightarrow k_{v}\right)$ sont des isomorphismes.

Démonstration. Soit $\mathbf{F}_{v}$ le corps résiduel de $\mathcal{O}_{v}$ et $\widetilde{G}$ la réduction de $\varphi$ modulo v. On a $H^{1}\left(\mathscr{O}_{v}, \mathscr{G}\right)=H^{1}\left(\mathbf{F}_{v}, \widetilde{G}\right)$; voir [Milne 1980], remarque 3.11a. D'autre part, comme $\widetilde{G}$ est connexe, on a $H^{1}\left(\mathbf{F}_{v}, \widetilde{G}\right)=0$ par le théorème de Lang, d'où la première assertion. La seconde assertion pour $i=0$ résulte de ce que $\mathscr{Y}$ est localement constant pour la topologie étale. Pour $i=1$, elle vient de [Harari et Szamuely 2005], p. 105.

Soit $v \notin S$. Alors $H^{0}\left(\mathcal{O}_{v}, \mathscr{G}\right)=\mathscr{G}\left(\mathcal{O}_{v}\right)$ s'injecte dans $G\left(k_{v}\right)$ et d'autre part le lemme précédent dit que $H^{i}\left(\mathscr{O}_{v}, \mathscr{Y}\right)$ est isomorphe à $H^{i}\left(k_{v}, Y\right)$ pour $i=0,1$; on voit donc par dévissage que l'application naturelle $H^{0}\left(\mathrm{O}_{v}, M\right) \rightarrow H^{0}\left(k_{v}, M\right)$ est injective, ce qui permet de noter encore $H^{0}\left(\mathcal{O}_{v}, \mathcal{M}\right)$ l'image de $H^{0}\left(\mathcal{O}_{v}, \mathcal{M}\right)$ dans $H^{0}\left(k_{v}, M\right)$. Notons que le groupe $H^{0}\left(\mathcal{O}_{v}, M\right)$ est quasi-compact (il possède un sous-groupe ouvert d'indice fini qui est un quotient de $\mathscr{G}\left(\mathcal{O}_{v}\right)$ ), mais pas séparé en général.

On pose ${ }^{1} H_{r}^{0}\left(k_{v}, M\right)=H^{0}\left(k_{v}, M\right) / H^{0}\left(\mathscr{O}_{v}, M\right)$ (avec une notation similaire si $M$ est remplacé par $T$ ) et on équipe ce groupe de la topologie quotient (pour la définition de la topologie sur $H^{0}\left(k_{v}, M\right)$, voir [Harari et Szamuely 2005], section 2).

Lemme 2. Soit $v \notin S$. Alors le groupe $H_{r}^{0}\left(k_{v}, M\right)$ est isomorphe à $H_{r}^{0}\left(k_{v}, T\right)$. $C^{\prime}$ 'est un groupe discret (autrement dit: $H^{0}\left(\mathrm{O}_{v}, M\right)$ est un sous-groupe ouvert de $\left.H^{0}\left(k_{v}, M\right)\right)$.

Notons qu'en particulier le groupe $H_{r}^{0}\left(k_{v}, M\right)$ est nul si $M=Y[1]$ ou $M=A$. Rappelons aussi qu'un sous-groupe d'un groupe topologique est ouvert si et seulement si le quotient topologique correspondant est un groupe discret.

Démonstration. D'après le lemme 1 , on a $H^{1}\left(\mathcal{O}_{v}, \mathscr{G}\right)=0$. On a alors un diagramme commutatif dont les lignes sont exactes :

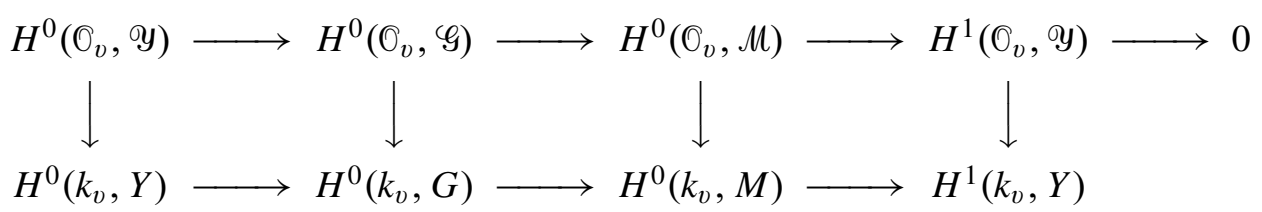

La première et la quatrième flèche verticale sont des isomorphismes via le lemme 1. Par chasse au diagramme on est ramené au cas $M=G$. Comme $H^{1}\left(\mathbb{O}_{v}, \mathscr{T}\right)=0$

1. L'indice $r$ signifie ici "ramifié" ; je remercie le rapporteur pour cette suggestion de notation. 
(lemme 1), on a un autre diagramme commutatif exact :

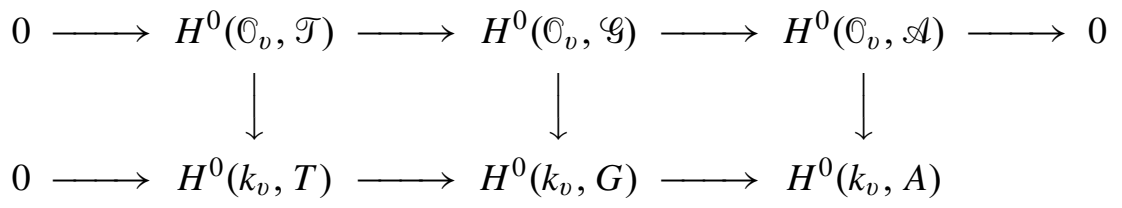

La flèche verticale de droite est un isomorphisme par propreté de $A$. Une nouvelle chasse au diagramme nous donne alors l'isomorphisme voulu.

Enfin, $H^{0}\left(\mathcal{O}_{v}, \mathscr{T}\right)$ est un sous-groupe ouvert de $H^{0}\left(k_{v}, T\right)$ parce que $\mathscr{O}_{v}$ est un sous-groupe ouvert de $k_{v}$ (prendre par exemple des équations affines de $\mathscr{T}$; voir aussi [Platonov et Rapinchuk 1994], p. 134), ce qui achève la démonstration du lemme.

Lemme 3. L'image de $H^{0}(k, M)$ dans $\mathbf{P}_{S}^{0}(M) / \prod_{v \notin S} H^{0}\left(\mathcal{O}_{v}, M\right)$ est d'indice fini.

Le groupe $\mathbf{P}_{S}^{0}(M) / \prod_{v \notin S} H^{0}\left(\mathcal{O}_{v}, \mathcal{M}\right)$ est isomorphe au groupe $\bigoplus_{v \notin S} H_{r}^{0}\left(k_{v}, M\right)$, qui est discret d'après le lemme 2. D'autre part, pour $M=\mathbf{G}_{m}$, le lemme 3 résulte juste de la finitude du groupe des classes d'idéaux de $\mathbb{O}$.

Démonstration. On a $H^{1}\left(\mathcal{O}_{v}, \mathscr{G}\right)=0$ via le lemme 1 , ce qui donne un diagramme commutatif exact :

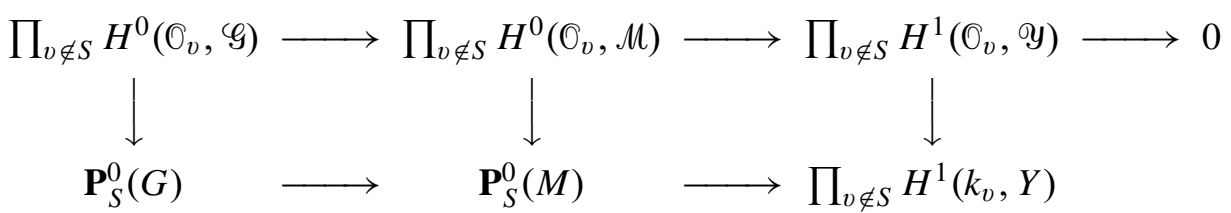

Comme on l'a déjà vu, la flèche verticale de droite est un isomorphisme; de ce fait $\mathbf{P}_{S}^{0}(G) / \prod_{v \notin S} H^{0}\left(\mathcal{O}_{v}, \mathscr{G}\right)$ se surjecte $\operatorname{sur} \mathbf{P}_{S}^{0}(M) / \prod_{v \notin S} H^{0}\left(\mathcal{O}_{v}, \mathcal{M}\right)$, ce qui permet de se ramener au cas $M=G$.

On écrit alors (en utilisant $H^{1}\left(\mathcal{O}_{v}, \mathscr{T}\right)=0$ ) le diagramme commutatif exact

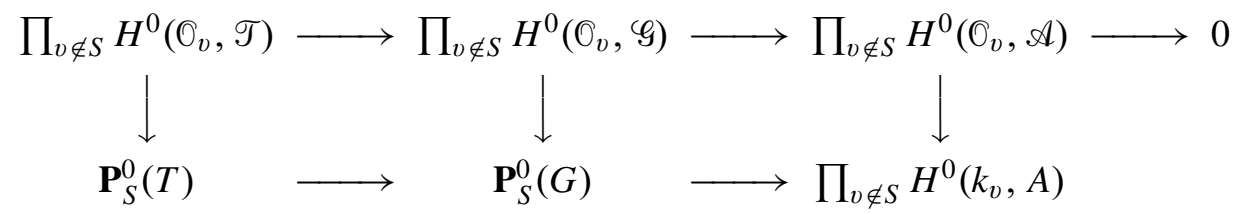

et la flèche verticale de droite est encore un isomorphisme. On est ainsi ramené au cas $M=T$, auquel cas le résultat vient de la finitude du groupe des classes d'idéaux d'un tore; voir [Platonov et Rapinchuk 1994], Theorem 5.1.

Proposition 1. On suppose que les cardinaux des groupes finis $\operatorname{ker}\left[H^{1}(U, \mathscr{T}) \rightarrow\right.$ $\left.H^{1}(k, T)\right]$ et $H^{1}(k, Y)$ sont premiers entre eux (par exemple si $\left.Y=0\right)$. Alors la suite de groupes discrets $0 \rightarrow H^{0}(U, M) \rightarrow H^{0}(k, M) \rightarrow \bigoplus H_{r}^{0}\left(k_{v}, M\right)$ est exacte. 
Notons que pour $U$ assez petit l'application $H^{1}(U, \mathscr{T}) \rightarrow H^{1}(k, T)$ est injective (voir [Harari et Szamuely 2005], Corrigenda) et l'hypothèse sur les cardinaux est alors vérifiée.

Démonstration. L'injectivité de $H^{0}(U, M) \rightarrow H^{0}(k, M)$ est démontrée dans [Harari et Szamuely 2005], Proposition 4.1(3). Le fait qu'on ait un complexe est clair car pour $v \notin S$, la flèche $H^{0}(U, M) \rightarrow H^{0}(k, M)$ se factorise par $H^{0}\left(\mathscr{O}_{v}, \mathcal{M}\right)$. Il reste donc à démontrer l'exactitude au milieu.

Montrons d'abord que le cardinal de $H^{1}(U, y)$ est premier avec celui de

$$
\operatorname{ker}\left[H^{1}(U, \mathscr{G}) \rightarrow H^{1}(k, G)\right] .
$$

Comme $H^{1}(U, \mathscr{A})$ s'injecte dans $H^{1}(k, A)$ (d'après [Milne 2006], lemme II.5.5) et $H^{0}(U, \mathscr{A}) \simeq H^{0}(k, A)$, le noyau de $H^{1}(U, \mathscr{T}) \rightarrow H^{1}(k, T)$ se surjecte sur celui de $H^{1}(U, \mathscr{G}) \rightarrow H^{1}(k, G)$. Mais la flèche $H^{1}(U, \mathscr{Y}) \rightarrow H^{1}(k, Y)$ est un isomorphisme (via [Harari et Szamuely 2005], diagramme p. 112) donc l'hypothèse faite sur le cardinal de $H^{1}(k, Y)$ donne le résultat voulu. Il en résulte que l'image de $H^{1}(U, \mathscr{Y}) \rightarrow H^{1}(U, \mathscr{G})$ s'injecte dans $H^{1}(k, G)$.

Maintenant, en utilisant le lemme 2, on obtient un diagramme commutatif, dont les lignes sont exactes :

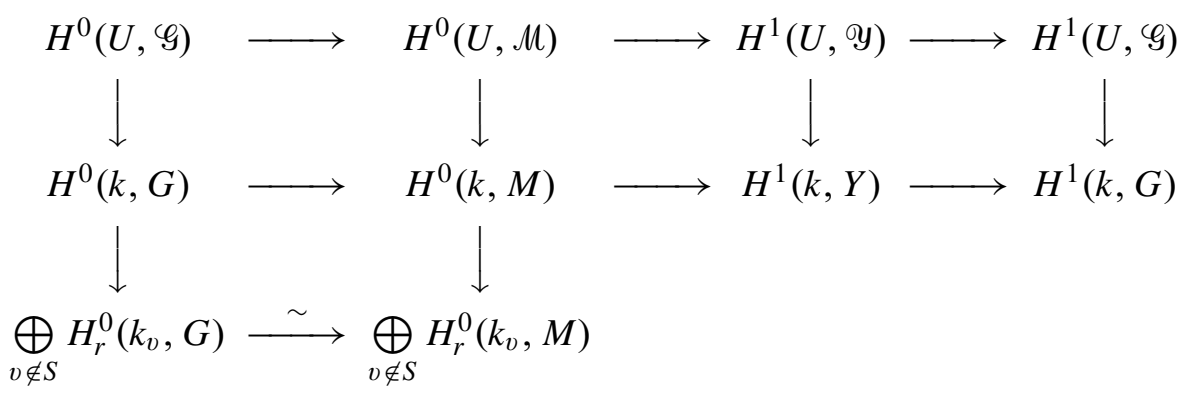

Une chasse au diagramme permet alors de se ramener au cas $M=G$. Or, dans ce cas le résultat est clair car un $k$-point de $G$ qui s'annule dans $\bigoplus_{v \notin S} H_{r}^{0}\left(k_{v}, G\right)$ correspond à un morphisme $\operatorname{Spec} k \rightarrow \mathscr{G}$ qui s'étend en tout point de codimension 1 du schéma de Dedekind $U$, donc sur $U$.

Corollaire 1. On suppose que les cardinaux de $H^{1}(k, Y)$ et $\operatorname{ker}\left[H^{1}(U, \mathcal{T}) \rightarrow\right.$ $\left.H^{1}(k, T)\right]$ sont premiers entre eux. Alors la suite

$$
H^{0}(U, M)^{\wedge} \rightarrow H^{0}(k, M)^{\wedge} \rightarrow\left[\bigoplus_{v \notin S} H_{r}^{0}\left(k_{v}, M\right)\right]^{\wedge}
$$

est exacte. 
Démonstration. D'après le lemme 3, l'image $I$ de $H^{0}(k, M)$ dans le groupe discret $\bigoplus_{v \notin S} H_{r}^{0}\left(k_{v}, M\right)$ est d'indice fini, ce qui implique que l'application induite $I^{\wedge} \rightarrow$ $\left[\bigoplus_{v \notin S} H_{r}^{0}\left(k_{v}, M\right)\right]^{\wedge}$ est injective. D'autre part la suite

$$
H^{0}(U, M)^{\wedge} \rightarrow H^{0}(k, M)^{\wedge} \rightarrow I^{\wedge} \rightarrow 0
$$

est exacte d'après la proposition 1 et [Harari et Szamuely 2005], appendice, (2). Le corollaire en résulte.

Rappelons que d'après le théorème 2.3 de [Harari et Szamuely 2005] on a un accouplement

$$
(\cdot, \cdot)_{v}: H^{0}\left(k_{v}, M\right) \times H^{1}\left(k_{v}, M^{*}\right) \rightarrow \mathbf{Q} / \mathbf{Z},
$$

lequel met en dualité parfaite le groupe discret $H^{1}\left(k_{v}, M^{*}\right)$ et le groupe profini $H^{0}\left(k_{v}, M\right)^{\wedge}$. Pour $v \notin S$, on sait aussi ([Harari et Szamuely 2005], début de la preuve du théorème 2.10) que cet accouplement s'annule sur $H^{0}\left(\mathbb{O}_{v}, M\right) \times$ $H^{1}\left(\mathrm{O}_{v}, M^{*}\right)$. Ceci nous permet de définir une flèche $\theta$ de $\mathbf{P}^{0}(M)$ dans le dual $H^{1}\left(k, M^{*}\right)^{D}$ de $H^{1}\left(k, M^{*}\right)$ par la formule

$$
\theta(x) . a=\sum_{v \in \Omega}\left(x_{v}, a_{v}\right)_{v}, \quad x \in \mathbf{P}^{0}(M), a \in H^{1}\left(k, M^{*}\right)
$$

où $x_{v}$ est la composante de $x$ en $v$ et $a_{v}$ la restriction de $a$ à $H^{1}\left(k_{v}, M^{*}\right)$ (qui est dans $H^{1}\left(\mathrm{O}_{v}, M^{*}\right)$ pour presque toute place $v$ ). On a de même une flèche (notée $\theta^{\wedge}$ ) de $\mathbf{P}^{0}(M)^{\wedge}$ dans $H^{1}\left(k, M^{*}\right)^{D}$.

Proposition 2. Supposons que les cardinaux de $H^{1}(k, Y)$ et $\operatorname{ker}\left[H^{1}(U, \mathscr{T}) \rightarrow\right.$ $\left.H^{1}(k, T)\right]$ soient premiers entre eux et que le groupe de Tate-Shafarevitch $\amalg(A)$ de A soit fini. Alors on a une suite exacte de groupes profinis

$$
H^{0}(U, M)^{\wedge} \rightarrow \mathscr{P}_{S}^{0}(M)^{\wedge} \stackrel{\theta}{\rightarrow} H^{1}\left(k, M^{*}\right)^{D}
$$

Démonstration. On écrit un diagramme commutatif :

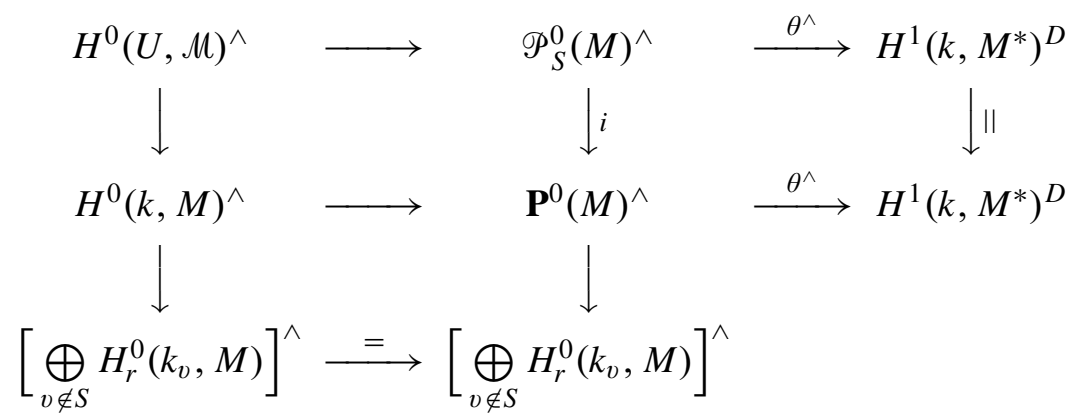

La première colonne est exacte $d$ 'après le corollaire 1 , la deuxième ligne est exacte d'après le théorème 5.6 de [Harari et Szamuely 2005] (ce qui implique déjà que la 
première ligne est un complexe), et la colonne du milieu est un complexe car c'est la complétion d'une suite exacte. Par chasse au diagramme, il suffit, pour montrer l'exactitude au milieu de la première ligne, de montrer que la flèche $i: \mathscr{P}_{S}^{0}(M)^{\wedge} \rightarrow$ $\mathbf{P}^{0}(M)^{\wedge}$ est injective.

La preuve du lemme 5.3. de [Harari et Szamuely 2005] donne que pour tout $n>0$, la flèche

$$
\mathscr{P}_{S}^{0}(M) / n \rightarrow \prod_{v \in \Omega} H^{0}\left(k_{v}, M\right) / n
$$

est injective. A fortiori la flèche

$$
\mathscr{P}_{S}^{0}(M) / n \rightarrow \mathbf{P}^{0}(M) / n
$$

est injective. Or $\mathscr{P}_{S}^{0}(M)^{\wedge}$ est la limite projective des $\mathscr{P}_{S}^{0}(M) / n$ car d'une part la complétion commute avec le produit, d'autre part on a

$$
H^{0}\left(k_{v}, M\right)^{\wedge}=\lim _{n} H^{0}\left(k_{v}, M\right) / n
$$

pour $v \in S$ et

$$
H^{0}\left(\mathcal{O}_{v}, M\right)^{\wedge}={\underset{\leftarrow}{n}}_{\lim ^{0}} H^{0}\left(\mathcal{O}_{v}, M\right) / n
$$

pour $v \notin S$ comme on le voit par dévissage en utilisant le fait que $H^{0}\left(k_{v}, T\right) / n$ et $H^{0}\left(\mathbb{O}_{v}, \mathscr{T}\right) / n$ sont finis, $H^{0}\left(k_{v}, A\right)=H^{0}\left(\mathscr{O}_{v}, \mathscr{A}\right)$ est profini, et $H^{1}\left(k_{v}, Y\right)=$ $H^{1}\left(\mathrm{O}_{v}\right.$, Y $)$ est fini ([Milne 2006], corollaire I.2.3). En passant à la limite projective, on obtient alors que $\mathscr{P}_{S}^{0}(M)^{\wedge}$ s'injecte dans la limite projective $\mathbf{P}^{0}(M)_{\wedge}$ des $\mathbf{P}^{0}(M) / n$. On conclut avec l'injectivité de la flèche $\mathbf{P}^{0}(M)_{\wedge} \rightarrow \mathbf{P}^{0}(M)^{\wedge}$; voir [Harari et Szamuely 2005], proposition 5.4 et diagramme (13).

Soit $\overline{H^{0}(U, M)}$ l'adhérence (pour la topologie produit) de $H^{0}(U, M)=H^{0}\left(\mathcal{O}_{S}, M\right)$ dans $\mathscr{P}_{S}^{0}(M)=\prod_{v \in S} H^{0}\left(k_{v}, M\right) \times \prod_{v \notin S} H^{0}\left(\mathcal{O}_{v}, M\right)$.

Théorème 1. Sous les hypothèses de la proposition 2, on a une suite exacte

$$
0 \rightarrow \overline{H^{0}(U, M)} \rightarrow \prod_{v \in S} H^{0}\left(k_{v}, M\right) \times \prod_{v \notin S} H^{0}\left(\mathcal{O}_{v}, M\right) \stackrel{\theta}{\rightarrow} H^{1}\left(k, M^{*}\right)^{D}
$$

Démonstration. La méthode est très similaire à celle de [Harari et Szamuely 2008], proposition 5.3. Le fait que la suite soit un complexe est clair par fonctorialité via le théorème 5.6 de [Harari et Szamuely 2005]. Soit $C$ le quotient topologique de $\mathscr{P}_{S}^{0}(M)$ par $\overline{H^{0}(U, M)}$. Le groupe $C$ est séparé car $\overline{H^{0}(U, M)}$ est fermé dans $\mathscr{P}_{S}^{0}(M)$. Montrons que $C$ s'injecte dans sa complétion profinie $C^{\wedge}$. D'après [Harari et Szamuely 2005], appendice, il suffit de vérifier que $C$ est engendré par une partie compacte. Or pour $v$ dans $S$, le groupe $H^{0}\left(k_{v}, M\right)$ a un sous-groupe d'indice fini qui est un quotient topologique de $G\left(k_{v}\right)$, lui-même engendré par une partie compacte via [Harari et Szamuely 2005], lemme 2.2; d'autre part pour $v \notin S$, le 
groupe $H^{0}\left(\mathcal{O}_{v}, M\right)$ est déjà quasi-compact. Ainsi $C$ est bien engendré par une partie compacte.

On écrit alors un diagramme commutatif :

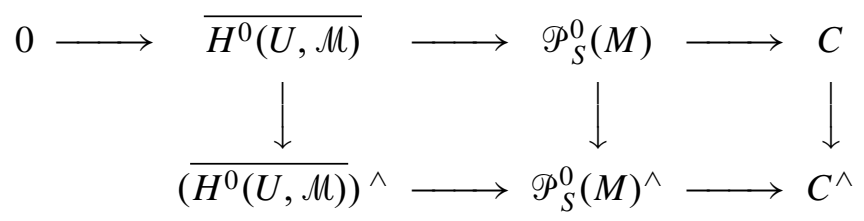

La première ligne est exacte par définition de $C$ et la deuxième ligne est un complexe par fonctorialité. Soit $x$ un élément de $\mathscr{P}_{S}^{0}(M)$ dont l'image dans $H^{1}\left(k, M^{*}\right)^{D}$ est nulle. D'après la proposition 2 , l'image $y$ de $x$ dans $\mathscr{P}_{S}^{0}(M)^{\wedge}$ provient de $H^{0}(U, M)^{\wedge}$, donc a fortiori de $\left(\overline{H^{0}(U, M)}\right)^{\wedge}$ vu que la flèche $H^{0}(U, M)^{\wedge} \rightarrow$ $\mathscr{P}_{S}^{0}(M)^{\wedge}$ se factorise par une flèche $H^{0}(U, M)^{\wedge} \rightarrow\left(\overline{H^{0}(U, M)}\right)^{\wedge}$. Ainsi, l'image de $y$ dans $C^{\wedge}$ est nulle et on conclut avec l'injectivité de $C \rightarrow C^{\wedge}$ et l'exactitude de la première ligne.

Remarque. Il est facile de voir directement à partir de la proposition 1 que le corollaire 1 reste valable en remplaçant la complétion ^ par la "complétion partielle" $\wedge=\lim _{\leftarrow} \cdot / n$. On obtient alors l'analogue (d'ailleurs plus simple à démontrer) de la proposition 2 avec cette complétion partielle grâce à la suite exacte (17) de [Harari et Szamuely 2005]. Cela suffit pour prouver le théorème 1 par la même méthode, tout en évitant les complications topologiques.

Lemme 4. Les groupes $\mathbf{P}^{0}(M)$ et $\mathbf{P}^{0}(M)^{\wedge}$ ont même image dans $H^{1}\left(k, M^{*}\right)^{D}$ par les applications respectives $\theta, \theta^{\wedge}$.

Démonstration. On procède par dévissage. Notons $\mathbf{P}^{1}(G)$ le produit restreint (pour $v \in \Omega$ ) des $H^{1}\left(k_{v}, G\right)$ relativement aux $H^{1}\left(\mathcal{O}_{v}, \mathscr{G}\right)$ (on emploiera de même les notations $\mathbf{P}^{1}(Y)$ et $\left.\mathbf{P}^{1}(T)\right)$. On a un diagramme commutatif exact

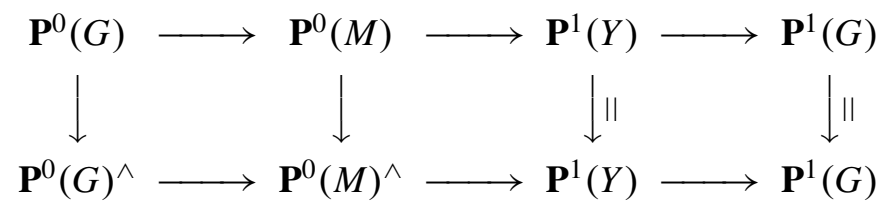

En effet, $\mathbf{P}^{1}(Y)=\prod_{v \in \Omega} H^{1}\left(k_{v}, Y\right)$ est déjà compact et la complétion de la première ligne reste donc exacte en vertu de [Harari et Szamuely 2005], appendice. Ainsi $\mathbf{P}^{0}(M)$ se surjecte sur le conoyau de $\mathbf{P}^{0}(G)^{\wedge} \rightarrow \mathbf{P}^{0}(M)^{\wedge}$ et on est ramené à $M=G$. On se ramène ensuite par le même argument au cas $M=T$ et $M^{*}=Y^{*}[1]$ 
en utilisant le diagramme commutatif exact

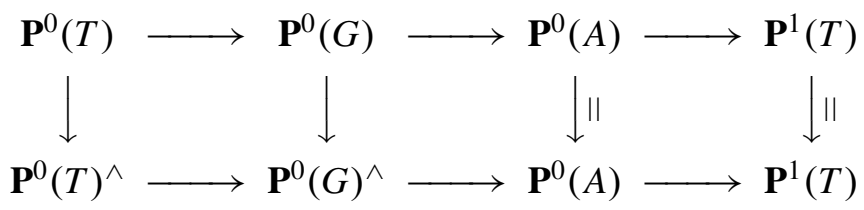

vu que $\mathbf{P}^{0}(A)=\prod_{v \in \Omega} H^{0}\left(k_{v}, A\right)$ est compact. Maintenant on observe que

$$
\mathbf{P}^{0}(T) / \overline{H^{0}(k, T)}
$$

est compact en vertu de la finitude du groupe des classes d'idéaux d'un tore (noter ici l'importance d'avoir considéré les $H^{0}$ modifiés à la Tate aux places archimédiennes). De ce fait l'image de $\mathbf{P}^{0}(T)$ dans le groupe profini $H^{1}\left(k, M^{*}\right)^{D}=$ $H^{2}\left(k, Y^{*}\right)^{D}$ est fermée; elle coïncide donc (par densité) avec l'image de $\mathbf{P}^{0}(T)^{\wedge}$.

Théorème 2. Soit $M$ un 1-motif sur $k$. On suppose que $\amalg(A)$ est fini et on note $\overline{H^{0}(k, M)}$ l'adhérence de $H^{0}(k, M)$ dans le produit restreint $\mathbf{P}^{0}(M)$. Alors on a une suite exacte

$$
0 \rightarrow \overline{H^{0}(k, M)} \rightarrow \mathbf{P}^{0}(M) \rightarrow H^{1}\left(k, M^{*}\right)^{D} \rightarrow \amalg^{1}(M) \rightarrow 0
$$

Ici la flèche $H^{1}\left(k, M^{*}\right)^{D} \rightarrow \amalg^{1}(M)$ est induite par la dualité parfaite entre les groupes finis $\amalg^{1}(M)$ et $\amalg^{1}\left(M^{*}\right)$, par le corollaire 4.9 de [Harari et Szamuely 2005].

Démonstration. On commence par fixer un ensemble fini $S$ de places tel que les hypothèses du théorème 1 soient satisfaites pour tout ouvert $U$ de $\operatorname{Spec}\left(O_{S}\right)$ (cf. commentaire après la proposition 1). Les trois premiers termes de la suite exacte s'obtiennent alors en appliquant le théorème 1 aux ensembles finis de places $T \supset$ $S$, et en faisant la limite inductive sur $T$. D'après le théorème 5.6 de [Harari et Szamuely 2005], la suite

$$
\mathbf{P}^{0}(M)^{\wedge} \rightarrow H^{1}\left(k, M^{*}\right)^{D} \rightarrow \amalg^{1}(M) \rightarrow 0
$$

est exacte. Comme $\mathbf{P}^{0}(M)$ et $\mathbf{P}^{0}(M)^{\wedge}$ ont même image dans $H^{1}\left(k, M^{*}\right)^{D}$ d'après le lemme précédent, le résultat est démontré.

Remarques. - On comparera avec le théorème 1.2 de [Harari et Szamuely 2008] : ici on considère l'adhérence de $H^{0}(k, M)$ pour une topologie de produit restreint (et non plus de produit direct), c'est-à-dire que le groupe $H^{1}\left(k, M^{*}\right)^{D}$ mesure le défaut d'approximation forte (tandis que le défaut d'approximation faible était mesuré par le groupe plus petit $\left.\amalg_{\omega}^{1}\left(M^{*}\right)^{D}\right)$. Bien entendu, si $M=A$ est une variété abélienne, il n'y a pas lieu de distinguer entre les deux résultats et on retrouve la classique suite exacte duale de Cassels-Tate ([Milne 2006], théorème I.6.26). 
- Le fait d'avoir considéré les $\widehat{H}^{0}$ aux places archimédiennes entraîne que même si $M$ est un tore, l'image de $H^{0}(k, M)$ dans $\mathbf{P}^{0}(M)$ n'est pas toujours fermée. Par exemple si $M=\mathbf{G}_{m}$ et $k=\mathbf{Q}(\sqrt{2})$, alors le sous-groupe $H$ de $\mathbf{P}^{0}\left(\mathbf{G}_{m}\right)$ engendré par l'image de $(1+\sqrt{2})$ et celle de $\{ \pm 1\}$ est isomorphe à $\mathbf{Z} \times \mathbf{Z} / 2 ;$ d'après le théorème de Baire, le groupe $H$ ne peut pas être compact car il est infini et dénombrable. Pourtant $H$ est l'intersection de l'image de $H^{0}\left(k, \mathbf{G}_{m}\right)=k^{*}$ avec le compact de $\mathbf{P}^{0}\left(\mathbf{G}_{m}\right)$ constitué des éléments dont toutes les composantes aux places $v$ non archimédiennes sont dans $\mathcal{O}_{v}^{*}$. Ainsi l'image de $k^{*}$ dans $\mathbf{P}^{0}\left(\mathbf{G}_{m}\right)$ ne peut pas être fermée.

- Dans le cas $M=\mathbf{G}_{m}$, le théorème 2 est essentiellement équivalent au cas où $S$ contient toutes les places du corollaire 8.3.21 p. 464 de [Neukirch et al. 2008]. Le théorème 1 est malgré les apparences d'une nature un peu différente (et semble être nouveau même pour $G=\mathbf{G}_{m}$ ).

\section{Une variante}

Le but de cette section est de démontrer une variante du théorème 1 qui fait intervenir deux ensembles finis de places, et d'en déduire un énoncé d'approximation pour les tores. On considère toujours $M=[Y \rightarrow G]$ un 1-motif sur $k$, qui s'étend en un 1-motif $M=\left[Y Y_{G} \rightarrow\right.$ au-dessus de $U=\operatorname{Spec}\left(O_{S}\right)$. Si $v$ est une place non dans $S$, on note $H_{\mathrm{nr}}^{1}\left(k_{v}, M\right)$ l'image de $H^{1}\left(\mathcal{O}_{v}, M\right)$ dans $H^{1}\left(k_{v}, M\right)$ (voir [Harari et Szamuely 2005], section 2). Rappelons qu'on a supposé que $S$ contenait toutes les places archimédiennes de $k$.

Soit $S^{\prime}$ un ensemble fini de places de $k$ avec $S \cap S^{\prime}=\varnothing$. On note $\overline{H^{0}\left(\mathcal{O}_{S}, M\right)} S^{\prime}$ l'adhérence de $H^{0}\left(\mathcal{O}_{S}, \mathcal{M}\right)=H^{0}(U, \mathcal{M})$ dans $\prod_{v \in S^{\prime}} H^{0}\left(\mathscr{O}_{v}, \mathcal{M}\right)$. On note aussi $H_{S, S^{\prime}}^{1}\left(k, M^{*}\right)$ l'ensemble des $\alpha$ de $H^{1}\left(k, M^{*}\right)$ vérifiant $\alpha_{v}=0$ pour $v \in S$ et $\alpha_{v} \in H_{\mathrm{nr}}^{1}\left(k_{v}, M^{*}\right)$ pour $v \notin\left(S \cup S^{\prime}\right)$. Enfin $H_{S, \Omega}^{1}\left(k, M^{*}\right)$ désigne l'ensemble des $\alpha$ de $H^{1}\left(k, M^{*}\right)$ vérifiant $\alpha_{v}=0$ pour $v \in S$ et $\alpha_{v} \in H_{\mathrm{nr}}^{1}\left(k_{v}, M^{*}\right)$ pour $v \notin S$.

Lemme 5. On garde les hypothèses de la proposition 2. Posons

$$
I=\prod_{v \in S} H^{0}\left(k_{v}, M\right)^{\wedge} \times \prod_{v \in S^{\prime}} H^{0}\left(\mathbb{O}_{v}, M\right) \times \prod_{v \notin\left(S \cup S^{\prime}\right)} H^{0}\left(\mathscr{O}_{v}, \mathcal{M}\right)^{\wedge}
$$

et notons abusivement $\overline{H^{0}(U, M)}$ l'adhérence de l'image de $H^{0}(U, M)$ dans $I$. Alors on a une suite exacte

$$
0 \rightarrow \overline{H^{0}(U, M)} \rightarrow I \stackrel{\theta}{\rightarrow} H^{1}\left(k, M^{*}\right)^{D}
$$

Notons que comme on va s'intéresser ensuite à une propriété d'approximation aux places de $S^{\prime}$, on n'a besoin ici d'enlever les completions pour I que dans le facteur du milieu. 
Démonstration. La preuve est tout à fait analogue (en plus simple) à celle du théorème 1 , en remplaçant $\mathscr{P}_{S}^{0}(M)$ par $I$ (qui est quasi-compact, ce qui fait que le quotient de $I$ par $\overline{H^{0}(U, M)}$ est déjà profini).

Proposition 3. On garde les hypothèses et notations de la proposition 2. Soit $S^{\prime}$ un ensemble fini de places de $k$ avec $S \cap S^{\prime}=\varnothing$. Alors on a une suite exacte

$$
0 \rightarrow{\overline{H^{0}\left(\mathcal{O}_{S}, \mathcal{M}\right)}}^{S^{\prime}} \rightarrow \prod_{v \in S^{\prime}} H^{0}\left(\mathcal{O}_{v}, \mu\right) \stackrel{\varphi}{\rightarrow} H_{S, S^{\prime}}^{1}\left(k, M^{*}\right)^{D} \rightarrow H_{S, \Omega}^{1}\left(k, M^{*}\right)^{D} \rightarrow 0 \quad(*)
$$

où l'application $\varphi$ est définie par

$$
\varphi\left(\left(x_{v}\right)\right) \cdot a=\sum_{v \in S^{\prime}}\left(x_{v}, a_{v}\right)_{v} \quad,\left(x_{v}\right) \in \prod_{v \in S^{\prime}} H^{0}\left(\mathcal{O}_{v}, \mathcal{M}\right) \quad, a \in H_{S, S^{\prime}}^{1}\left(k, M^{*}\right)^{D}
$$

Démonstration. On note d'abord que le quotient topologique

$$
\prod_{v \in S^{\prime}} H^{0}\left(\mathscr{O}_{v}, \mu\right) /{\overline{H^{0}\left(\mathscr{O}_{S}, M\right)}}^{\prime}
$$

est séparé et quasi-compact, donc compact. De ce fait

$$
\prod_{v \in S^{\prime}} H^{0}\left(\mathcal{O}_{v}, M\right) \text { et } \prod_{v \in S^{\prime}} H^{0}\left(\mathcal{O}_{v}, M\right)^{\wedge}
$$

ont même image dans $H_{S, S^{\prime}}^{1}\left(k, M^{*}\right)^{D}$. On obtient l'exactitude des trois derniers termes de $(*)$ avec le théorème 2.10 de [Harari et Szamuely 2005], en dualisant la suite (qui est exacte par définition) :

$$
0 \rightarrow H_{S, \Omega}^{1}\left(k, M^{*}\right) \rightarrow H_{S, S^{\prime}}^{1}\left(k, M^{*}\right) \rightarrow \prod_{v \in S^{\prime}} H^{1}\left(k_{v}, M^{*}\right) / H_{\mathrm{nr}}^{1}\left(k_{v}, M^{*}\right)
$$

D'autre part les trois premiers termes de $(*)$ forment un complexe vu que l'accouplement local entre $H^{0}\left(\mathcal{O}_{v}, M\right)$ et $H_{\mathrm{nr}}^{1}\left(k_{v}, M^{*}\right)$ est trivial.

Soit donc $\left(x_{v}\right)_{v \in S^{\prime}}$ dans $\prod_{v \in S^{\prime}} H^{0}\left(\mathscr{O}_{v}, \mathcal{M}\right)$, d'image nulle par $\varphi$. D'après le lemme 5 , il suffit de montrer qu'il existe une famille

$$
\left(x_{v}\right) \in \prod_{v \notin\left(S \cup S^{\prime}\right)} H^{0}\left(\mathcal{O}_{v}, M\right)^{\wedge}
$$

et une famille

$$
\left(x_{v}\right) \in \prod_{v \in S} H^{0}\left(k_{v}, M\right)^{\wedge}
$$

vérifiant : l'image de la famille $\left(x_{v}\right)_{v \in \Omega}$ dans $H^{1}\left(k, M^{*}\right)^{D}$ (par la flèche $\theta$ du lemme $5)$ est nulle. 
Soit $N$ le dual du groupe

$$
\bigoplus_{v \in S} H^{1}\left(k_{v}, M^{*}\right) \oplus \bigoplus_{v \notin\left(S \cup S^{\prime}\right)}\left(H^{1}\left(k_{v}, M^{*}\right) / H_{\mathrm{nr}}^{1}\left(k_{v}, M^{*}\right)\right)
$$

et posons

$$
N^{\prime}:=\prod_{v \in S} H^{0}\left(k_{v}, M\right)^{\wedge} \times \prod_{v \notin\left(S \cup S^{\prime}\right)} H^{0}\left(\mathscr{O}_{v}, M\right)^{\wedge}
$$

On a alors un diagramme commutatif dont les colonnes sont exactes :

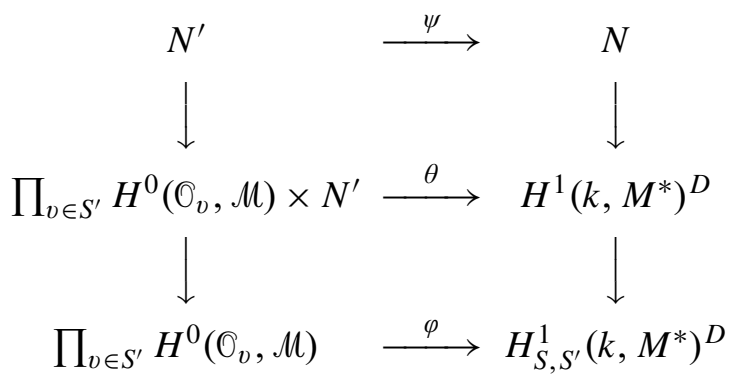

Comme $\psi$ est un isomorphisme par dualité locale, on conclut par chasse au diagramme, en considérant l'élément de

$$
I=\prod_{v \in S^{\prime}} H^{0}\left(\mathscr{O}_{v}, \mathcal{M}\right) \times N^{\prime}
$$

obtenu en complétant $\left(x_{v}\right)_{v \in S^{\prime}}$ avec des zéros.

Soient maintenant $T$ un $k$-tore de module des caractères $Y$, et $S_{0}$ un ensemble fini de places de $k$ (contenant les places archimédiennes) tel que les 1-motifs $M=$ $[0 \rightarrow T]$ et $M^{*}=[Y \rightarrow 0]$ s'étendent en des 1-motifs $\mathcal{M}$ et $\mathcal{M}^{*}=[Y \rightarrow 0]$ au-dessus de $\operatorname{Spec}\left(O_{S_{0}}\right)$. On note $\amalg_{\omega}^{2}(Y)$ l'ensemble des éléments $\alpha$ de $H^{2}(k, Y)$ tels que la localisation $\alpha_{v} \in H^{2}\left(k_{v}, Y\right)$ soit nulle pour presque toute place $v$ de $k$.

Théorème 3. Avec les notations ci-dessus, on suppose que $\amalg_{\omega}^{2}(Y)=0$. Soit $S^{\prime}$ un ensemble fini de places de $k$ ne rencontrant pas $S_{0}$. Alors il existe un ensemble fini de places $S$ avec $S \supset S_{0}$ et $S \cap S^{\prime}=\varnothing$, vérifiant : $T\left(\mathcal{O}_{S}\right)$ est dense dans $\prod_{v \in S^{\prime}} T\left(\mathcal{O}_{v}\right)$.

Démonstration. La structure des groupes de Lie $p$-adiques compacts donne qu'il existe un ensemble fini $\Sigma$ de nombres premiers tels que pour $\ell \notin \Sigma$, le groupe $\prod_{v \in S^{\prime}} T\left(\mathcal{O}_{v}\right)$ soit $\ell$-divisible. D'après la proposition 3 , il suffit alors de trouver $S$ tel que la $\ell$-torsion $H_{S, S^{\prime}}^{2}(k, Y)[\ell]$ du groupe $H_{S, S^{\prime}}^{2}(k, Y)$ soit nulle pour tout $\ell$ de $\Sigma$. En utilisant la suite exacte de $\mathscr{O}_{S^{\prime} \cup S_{0}}$-schémas en groupes lisses

$$
0 \rightarrow \mathrm{y} \stackrel{l}{\rightarrow} \mathrm{y} \rightarrow \mathrm{y} / \ell \rightarrow 0
$$

on voit que $H^{1}\left(\mathcal{O}_{S^{\prime} \cup S_{0}}\right.$, y $\left./ \ell\right)$ (dont l'image dans $H^{1}(k, Y / \ell)$ est finie via [Serre 1994], II.6.2., théorème 7) se surjecte sur $H^{2}\left(\mathcal{O}_{S^{\prime} \cup S_{0}}\right.$, Y $)[\ell]$. De ce fait il n'y a 
qu'un nombre fini d'éléments non nuls de $H^{2}(k, Y)[\ell]$ qui sont non ramifiés en dehors de $\left(S_{0} \cup S^{\prime}\right)$; pour chacun de ces éléments $\alpha$, on peut choisir une place $v$ non dans $\left(S_{0} \cup S^{\prime}\right)$ avec $\alpha_{v} \neq 0$ (grâce à l'hypothèse $\amalg_{\omega}^{2}(Y)=0$ ). Il suffit alors de prendre pour $S$ la réunion de $S_{0}$ et de toutes ces places $v$.

Remarques. - Il n'est pas étonnant de voir apparaître ici l'hypothèse $\amalg_{\omega}^{2}(Y)=0$, qui permet ([Sansuc 1981], théorème 8.12) d'avoir l'approximation faible pour $T$ (dont le théorème 3 est un raffinement).

- Il n'est pas raisonnable d'attendre un analogue du théorème 3 pour une variété semi-abélienne $G$. En effet si $G$ s'écrit comme extension d'une variété abélienne non nulle $A$ par un tore $T$ (dont on note encore $Y$ le module des caractères), alors $H^{1}\left(k, A^{*}\right)$ est infini donc aussi son image dans $H^{1}\left(k, M^{*}\right)$ (en effet $H^{1}(k, Y)$ est fini). Or cette image est incluse dans $\amalg_{\omega}^{1}\left(M^{*}\right)$ : en effet toute classe de $H^{1}\left(k, A^{*}\right)$ est non ramifiée en dehors d'un nombre fini de places (donc nulle en dehors de ces places par connexité de $A$, voir lemme 1). Il en résulte que $\amalg_{\omega}^{1}\left(M^{*}\right)$ est infini, donc que (modulo finitude de $\amalg(A)$ ) le défaut d'approximation faible pour $G$ l'est également via [Harari et Szamuely 2008], théorème 1.2.

- On comparera le théorème 3 avec les résultats de [Naumann 2008], section 4.

\section{Application à l'obstruction de Manin pour les points entiers sur les torseurs}

Rappelons ([Skorobogatov 2001], chapitre 5) que si $X$ est une $k$-variété algébrique, on a un accouplement de Brauer-Manin

$$
X\left(\mathbf{A}_{k}\right) \times \operatorname{Br} X \rightarrow \mathbf{Q} / \mathbf{Z}
$$

entre les points adéliques de $X$ et son groupe de Brauer. Le noyau à gauche de cet accouplement contient l'ensemble $X(k)$ des points rationnels de $X$. Soit $\mathscr{X}$ un modèle entier de $X$. On peut se demander si pour certaines classes de variétés, l'existence d'un point de $\mathscr{L}\left(\mathbf{A}_{\odot}\right)$ dans le noyau ci-dessus implique l'existence d'un point entier sur $X$. Nous allons montrer qu'il en est ainsi si $X$ est un espace principal homogène d'une variété semi-abélienne. Notons que cette problématique de l'obstruction de Manin entière a été formalisée pour la première fois dans [ColliotThélène et Fei 2007].

Théorème 4. Soit $\mathscr{X}$ un $\mathrm{O}$-schéma plat dont la fibre générique $X$ est un espace principal homogène sous une variété semi-abélienne $G$. On suppose que le groupe de Tate-Shafarevitch $\amalg(A)$ du quotient abélien A de G est fini.

Soit $S$ un ensemble fini de places de $k$, contenant les places archimédiennes. On suppose qu'il existe un point adélique $\left(P_{v}\right) \in \mathscr{L}\left(\mathbf{A}_{S}\right)$ qui est orthogonal à $\mathrm{Br}_{1} X$ pour l'accouplement de Brauer-Manin. Alors il existe un point de $\mathscr{X}\left(\mathbb{O}_{S}\right)$ qui est 
arbitrairement proche de $P_{v}$ pour $v \in S$ non archimédienne, et dans la même composante connexe de $X\left(k_{v}\right)$ que $P_{v}$ pour v réelle. En particulier, si on suppose de plus $\left(P_{v}\right) \in \mathscr{L}\left(\mathbf{A}_{\odot}\right)$, alors $\mathscr{L}(\mathcal{O}) \neq \varnothing$.

Démonstration. La fibre générique $X$ possède un point adélique orthogonal à $\mathrm{Br}_{1} X$ pour l'accouplement de Brauer-Manin. D'après [Harari 2006], théorème 1 (voir aussi [Harari et Szamuely 2008], théorème 1.1), $X$ possède un point rationnel; ceci implique qu'il existe un $k$-isomorphisme $\varphi: X \simeq G$. Quitte à agrandir $S$ on peut supposer que $\mathscr{G}_{S}$ s'étend en un schéma en groupes lisses $\mathscr{G}$ au-dessus de $\mathcal{O}_{S}$, et que $\varphi$ s'étend en un $\mathscr{O}_{S}$-isomorphisme $\varphi_{S}: \mathscr{L}_{S} \rightarrow \mathscr{G}_{S}$, où l'on a posé $\mathscr{L}_{S}=\mathscr{X} \times \times_{\mathbb{O}} \mathscr{O}_{S}$ (notation similaire pour $\mathscr{G}$ ).

Considérons alors le point $\left(Q_{v}\right) \in \mathscr{G}\left(\mathbf{A}_{S}\right)$ défini par $Q_{v}=\varphi\left(P_{v}\right)$ pour toute place $v$ de $k$. Notons $\left(\widetilde{Q}_{v}\right) \in \mathscr{P}_{S}^{0}(G)=\prod_{v \in S} H^{0}\left(k_{v}, G\right) \times \prod_{v \notin S} H^{0}\left(\mathcal{O}_{v}, \mathscr{G}\right)$ l'image de $\left(Q_{v}\right)$. Par fonctorialité de l'accouplement de Brauer-Manin, on obtient que $\left(Q_{v}\right)$ est orthogonal à $\mathrm{Br}_{1} G$. Posons $M=[0 \rightarrow G]$ et notons $M^{*}$ le 1-motif dual. On a construit dans [Harari et Szamuely 2008] (section 4) une flèche

$$
\imath: H^{1}\left(k, M^{*}\right) \rightarrow \mathrm{Br}_{1} G / \mathrm{Br} k
$$

qui est compatible (voir [Harari et Szamuely 2008], p. 22) avec l'accouplement de Brauer-Manin et les accouplements locaux, c'est-à-dire qu'on a, pour tout $\alpha \in$ $H^{1}\left(k, M^{*}\right)$ :

$$
\sum_{v \in \Omega}\left(\alpha_{v}, \widetilde{Q}_{v}\right)_{v}=\left(l(\alpha), Q_{v}\right)_{B M}
$$

où $(., .)_{B M}$ est l'accouplement de Brauer-Manin et $(., .)_{v}$ est l'accouplement local $H^{1}\left(k_{v}, M^{*}\right) \times H^{0}\left(k_{v}, M\right) \rightarrow \mathbf{Q} / \mathbf{Z}$.

Il en résulte que $\widetilde{Q}_{v}$ est dans le noyau de la flèche

$$
\mathscr{P}_{S}^{0}(G) \rightarrow H^{1}\left(k, M^{*}\right)^{D}
$$

du théorème 1 . Ce théorème dit alors que $\left(\widetilde{Q}_{v}\right)$ est dans $\overline{H^{0}\left(\mathscr{O}_{S}, \mathscr{G}\right)}$. Cela implique

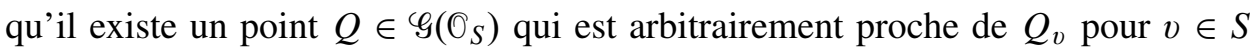
non archimédienne, et dans la même composante connexe de $G\left(k_{v}\right)$ que $Q_{v}$ pour $v$ réelle. Il suffit alors de poser $P_{v}=\varphi^{-1}\left(Q_{v}\right)$.

Remarques. - Dans le cas où $G=A$ est une variété abélienne, le théorème 4 (dû dans ce cas à Y. Manin et L. Wang) est essentiellement une reformulation de la suite exacte duale de Cassels-Tate. Quand $G=T$ est un tore, on obtient en particulier que l'obstruction de Manin à l'existence d'un point entier est la seule pour un $\mathbf{0}-$ schéma dont la fibre générique est un torseur sous un k-tore. On comparera avec les théorèmes 3.7. et 4.5. de [Colliot-Thélène et Fei 2007]. Notons aussi que le théorème 4 a surtout un intérêt théorique dans la mesure où déjà pour un tore $T$, le 
groupe $\mathrm{Br}_{1} T / \mathrm{Br} k$ est infini, donc l'obstruction a peu de chances d'être calculable en pratique.

- Ceci dit, si $\mathscr{X}(\mathcal{O})$ est vide, seul un nombre fini d'éléments de $\mathrm{Br} X$ est nécessaire pour obtenir une obstruction de Brauer-Manin entière. En effet le produit $\mathscr{P}$ des $\mathscr{L}\left(\mathrm{O}_{v}\right)$ est compact (en convenant que $\mathscr{X}\left(\mathcal{O}_{v}\right)$ est l'ensemble des composantes connexes de $X\left(k_{v}\right)$ si $v$ est archimédienne). Si on note $E_{\alpha}$ (pour $\alpha$ dans $\operatorname{Br} X$ ) le fermé de $\mathscr{P}$ où $\alpha$ s'annule, alors la condition $\mathscr{L}(\mathcal{O})=\varnothing$ implique (d'après le théorème 4 ) que l'intersection de tous les $E_{\alpha}$ est vide, donc par compacité qu'une intersection finie de tels $E_{\alpha}$ est vide (remarque due à Colliot-Thélène).

En appliquant le théorème 4 au cas où $G$ est un tore normique de dimension 1 , on obtient :

Exemple 1. Soit $\mathscr{X}$ une conique affine d'équation

$$
a x^{2}+b y^{2}=c \quad a, b, c \in \mathcal{O} \quad a b c \neq 0
$$

On note $X$ la conique affine sur $k$ associée. S'il existe pour toute place $v$ de $k$ une solution $\left(x_{v}, y_{v}\right)$ dans $\mathbb{O}_{v} \times \mathcal{O}_{v}$, telle que le point adélique $\left(P_{v}\right)=\left(x_{v}, y_{v}\right)_{v \in \Omega}$ soit orthogonal à $\mathrm{Br}_{1} X / \mathrm{Br} k$, alors il existe une solution $(x, y) \in \mathbb{O} \times \mathbb{O}$.

Ceci répond à une question posée par Colliot-Thélène. Notons que même le cas où $-b / a$ est un carré dans $k^{*}$ (qui correspond au cas $G=\mathbf{G}_{m}$ dans le théorème 4) n'est pas trivial.

\section{Remerciements}

Ce travail a pour origine plusieurs discussions avec J-L. Colliot-Thélène, et a bénéficié de ses commentaires; je l'en remercie chaleureusement. Je remercie également le rapporteur pour plusieurs suggestions pertinentes.

\section{Références}

[Colliot-Thélène et Fei 2007] J.-L. Colliot-Thélène et X. Fei, "Brauer-Manin obstruction for integral points of homogeneous spaces and representation by integral quadratic forms", prépublication, 2007, Voir http ://www.math.u-psud.fr/ colliot/CTXuFei4feb08.pdf.

[Harari 2006] D. Harari, “The Manin obstruction for torsors under connected algebraic groups”, Int. Math. Res. Not. 2006 (2006), Art. ID 68632. MR 2007b :14039 Zbl 1100.14013

[Harari et Szamuely 2005] D. Harari et T. Szamuely, "Arithmetic duality theorems for 1-motives", J. Reine Angew. Math. 578 (2005), 93-128. MR 2006f :14053 Zbl 1099.14008

[Harari et Szamuely 2008] D. Harari et T. Szamuely, "Local-global principles for 1-motives", Duke Math. J. 143 :3 (2008), 531-557.

[Milne 1980] J. S. Milne, Étale cohomology, Princeton Mathematical Series 33, Princeton University Press, Princeton, NJ, 1980. MR 81j:14002 Zbl 0433.14012 
[Milne 2006] J. S. Milne, Arithmetic duality theorems, Second éd., BookSurge, LLC, Charleston, SC, 2006. MR 2007e :14029 Zbl 1127.14001

[Naumann 2008] N. Naumann, "Arithmetically defined dense subgroups of Morava stabilizer groups", Compos. Math. 144 :1 (2008), 247-270. MR 2388563 Zbl 1134.55006

[Neukirch et al. 2008] J. Neukirch, A. Schmidt et K. Wingberg, Cohomology of number fields, 2nd éd., Grundlehren der Math. Wiss. 323, Springer, Berlin, 2008. MR 2392026 Zbl 1136.11001

[Platonov et Rapinchuk 1994] V. Platonov et A. Rapinchuk, Algebraic groups and number theory, Pure and Applied Math. 139, Academic Press, Boston, 1994. MR 95b :11039 Zbl 0841.20046

[Sansuc 1981] J.-J. Sansuc, "Groupe de Brauer et arithmétique des groupes algébriques linéaires sur un corps de nombres", J. Reine Angew. Math. 327 (1981), 12-80. MR 83d :12010 Zbl 0468.14007

[Serre 1994] J.-P. Serre, Cohomologie galoisienne, 5ème éd., Lecture Notes in Mathematics 5, Springer, Berlin, 1994. MR 96b :12010 Zbl 0812.12002

[Skorobogatov 2001] A. Skorobogatov, Torsors and rational points, Cambridge Tracts in Mathematics 144, Cambridge University Press, Cambridge, 2001. MR 2002d :14032 Zbl 0972.14015

Communicated by Bjorn Poonen

Received 2008-04-22 Revised 2008-05-26 Accepted 2008-06-26

David.Harari@math.u-psud.fr Université Paris-Sud, Laboratoire de Mathématiques d'Orsay, F-91405 Orsay Cedex, France 


\section{Guidelines for Authors}

Authors may submit manuscripts in PDF format on-line at the Submission page at the ANT website.

Originality. Submission of a manuscript acknowledges that the manuscript is original and and is not, in whole or in part, published or under consideration for publication elsewhere. It is understood also that the manuscript will not be submitted elsewhere while under consideration for publication in this journal.

Language. Articles in ANT are usually in English, but articles written in other languages are welcome.

Required items. A brief abstract of about 150 words or less must be included. It should be self-contained and not make any reference to the bibliography. If the article is not in English, two versions of the abstract must be included, one in the language of the article and one in English. Also required are keywords and subject classifications for the article, and, for each author, postal address, affiliation (if appropriate), and email address.

Format. Authors are encouraged to use $\mathrm{LTT}_{\mathrm{E} X}$ but submissions in other varieties of $\mathrm{T}_{\mathrm{E}} \mathrm{X}$, and exceptionally in other formats, are acceptable. Initial uploads should be in PDF format; after the refereeing process we will ask you to submit all source material.

References. Bibliographical references should be complete, including article titles and page ranges. All references in the bibliography should be cited in the text. The use of $\mathrm{BibT}_{\mathrm{E}} \mathrm{X}$ is preferred but not required. Tags will be converted to the house format, however, for submission you may use the format of your choice. Links will be provided to all literature with known web locations and authors are encouraged to provide their own links in addition to those supplied in the editorial process.

Figures. Figures must be of publication quality. After acceptance, you will need to submit the original source files in vector graphics format for all diagrams in your manuscript: vector EPS or vector PDF files are the most useful.

Most drawing and graphing packages (Mathematica, Adobe Illustrator, Corel Draw, MAT$\mathrm{LAB}$, etc.) allow the user to save files in one of these formats. Make sure that what you are saving is vector graphics and not a bitmap. If you need help, please write to graphics@mathscipub.org with details about how your graphics were generated.

White Space. Forced line breaks or page breaks should not be inserted in the document. There is no point in your trying to optimize line and page breaks in the original manuscript. The manuscript will be reformatted to use the journal's preferred fonts and layout.

Proofs. Page proofs will be made available to authors (or to the designated corresponding author) at a Web site in PDF format. Failure to acknowledge the receipt of proofs or to return corrections within the requested deadline may cause publication to be postponed. 


\section{Algebra \& Number Theory}

Volume $2 \quad$ No. $5 \quad 2008$

Homology and cohomology of quantum complete intersections

PETTER ANDREAS BERGH and KARIN ERDMANN

Tate resolutions for Segre embeddings

DAVID A. COX and EVGENy MATEROV

Constructing simply laced Lie algebras from extremal elements

JAN DRAISMA and JOS IN 'T PANHUIS

Integral traces of singular values of weak Maass forms

William DUKE and PAUl JENKINS

Le défaut d'approximation forte pour les groupes algébriques commutatifs

DAVID HARARI 